\title{
Rhodium-Catalyzed Nitrene/Alkyne Metathesis: An Enantioselective Process for the Synthesis of $N$-Heterocycles
}

\author{
Kemiao Hong, ${ }^{\dagger}$ Han $\mathrm{Su},{ }^{\dagger}$ Chao Pei,${ }^{\dagger}$ Xinxin Lv, ${ }^{\ddagger}$ Wenhao Hu, ${ }^{\dagger}$ Lihua Qiu,,${ }^{* \dagger}$ and \\ Xinfang $\mathrm{Xu}^{*}, \dagger,+$
}

${ }^{\dagger}$ College of Chemistry, Chemical Engineering and Materials Science, Soochow University, Suzhou 215123, China

${ }^{+}$Guangdong Key Laboratory of Chiral Molecule and Drug Discovery, School of Pharmaceutical Sciences, Sun Yat-sen University, Guangzhou, Guangdong 510006, China

$$
\begin{aligned}
& \text { E-mail: qiulihua@suda.edu.cn } \\
& \text { xinfangxu@suda.edu.cn }
\end{aligned}
$$

\section{Table of Contents}

1. General Information

2. Condition Optimization

2. General Procedure for the Synthesis of Sulfamate Esters 1

3. General Procedure for the Asymmetric Cascade Reaction

4. General Procedure for Scale Up and Derivatizations

5. 1D-noe NMR Analysis of $2 \mathrm{w}, 3$, and $5 \mathrm{~s}$

6. References

7. NMR Spectra for $2,3,4,5$, and 6

8. HPLC Analyses Figures for 2, 3, 4, 5, and 6

S72-S101

9. Single-Crystal X-ray Diffraction of 2d 


\section{General Information}

All reactions were carried out in oven-dried glassware. Solvents were dried by following the standard methods. Flash column chromatography was performed using silica gel (300-400 mesh). Analytical thin-layer chromatography was performed using glass plates pre-coated with 200-300 mesh silica gel impregnated with a fluorescent indicator $(254 \mathrm{~nm}) .{ }^{1} \mathrm{H}$ NMR and ${ }^{13} \mathrm{C}$ NMR spectra were recorded in $\mathrm{CDCl}_{3}$ on a 400 $\mathrm{MHz}$ spectrometer; chemical shifts were reported in ppm with the solvent signal as reference, and coupling constants $(J)$ were given in Hertz. The peak information was described as: $\mathrm{br}=$ broad, $\mathrm{s}=$ singlet, $\mathrm{d}=$ doublet, $\mathrm{t}=$ triplet, $\mathrm{q}=$ quartet, $\mathrm{m}=$ multiplet, comp $=$ composite. Enantioselectivity was determined on HPLC using Chiralpak IA-3 column, Daicel Chiralcel IA and IB column and Daicel Chiralpak AD-H column. High-resolution mass spectra (HRMS) were recorded on a commercial apparatus (ESI Source) and (CI Source).

\section{Condition Optimization}

We began our investigation of the designed nitrene/alkyne metathesis (NAM) cascade reaction with alkyne-tethered sulfamate 1a (Table S1). The racemic product 2a was obtained in $85 \%$ isolated yields when the reaction was catalyzed by $\mathrm{Du}$ Bois' $\mathrm{Rh}_{2}(\mathrm{esp})_{2}$ catalyst in dichloromethane $(\mathrm{DCM})$ at room temperature (entry 1). Encouraged by these results, a variety of chiral dirhodium catalysts were investigated (entries 2-12). All these tested catalysts gave the desired product in high yields (61-81\% yields), and superior results in term of the enantioselectivty were observed when using chiral catalyst $\mathrm{Rh}_{2}(S \text {-TCPTTL })_{4}$ with 1.0 mol\% loading (entry 5, 72\% ee). Screening of solvents in the presence of $\mathrm{Rh}_{2}(S \text {-TCPTTL })_{4}$ revealed that the enantioselectivity was improved more than $10 \%$ when the reaction was performed in toluene, chlorobenzene, or trifluorotoluene, and generating $\mathbf{2 a}$ in $>64 \%$ isolated yield with $83 \%-84 \%$ ee (entries 14-16). No reaction occurred when the reaction was conducted in methyl tert-butyl ether (TBME) due to the very low solubility (entry 17). 
Further optimization of the reaction temperature and conditions in trifluorotoluene turned out that the reaction gave the better results at $0{ }^{\circ} \mathrm{C}$ in the presence of $4 \AA$ molecular sieve with prolonged reaction time (entry $21,71 \%$ yield and $90 \% e e$ ). The best results were obtained using mixed solvent, $\mathrm{CF}_{3} \mathrm{C}_{6} \mathrm{H}_{5} / \mathrm{TBME}=10: 1$, which provided $77 \%$ yield with $92 \%$ ee after running the reaction for 60 hours (entry 22). It is worth mentioning that the only catalyst involved in this four-step cascade transformation, the rhodium-nitrene formation/NAM process/oxonium ylide formation/[2,3]-sigmatropic rearrangement sequence, is a chiral dirhodium catalyst, and this is the first example of catalytic asymmetric cascade reaction of $\alpha$-imino carbene intermediate generated in situ through the NAM process.

\section{Table S1. Condition Optimization ${ }^{a}$}

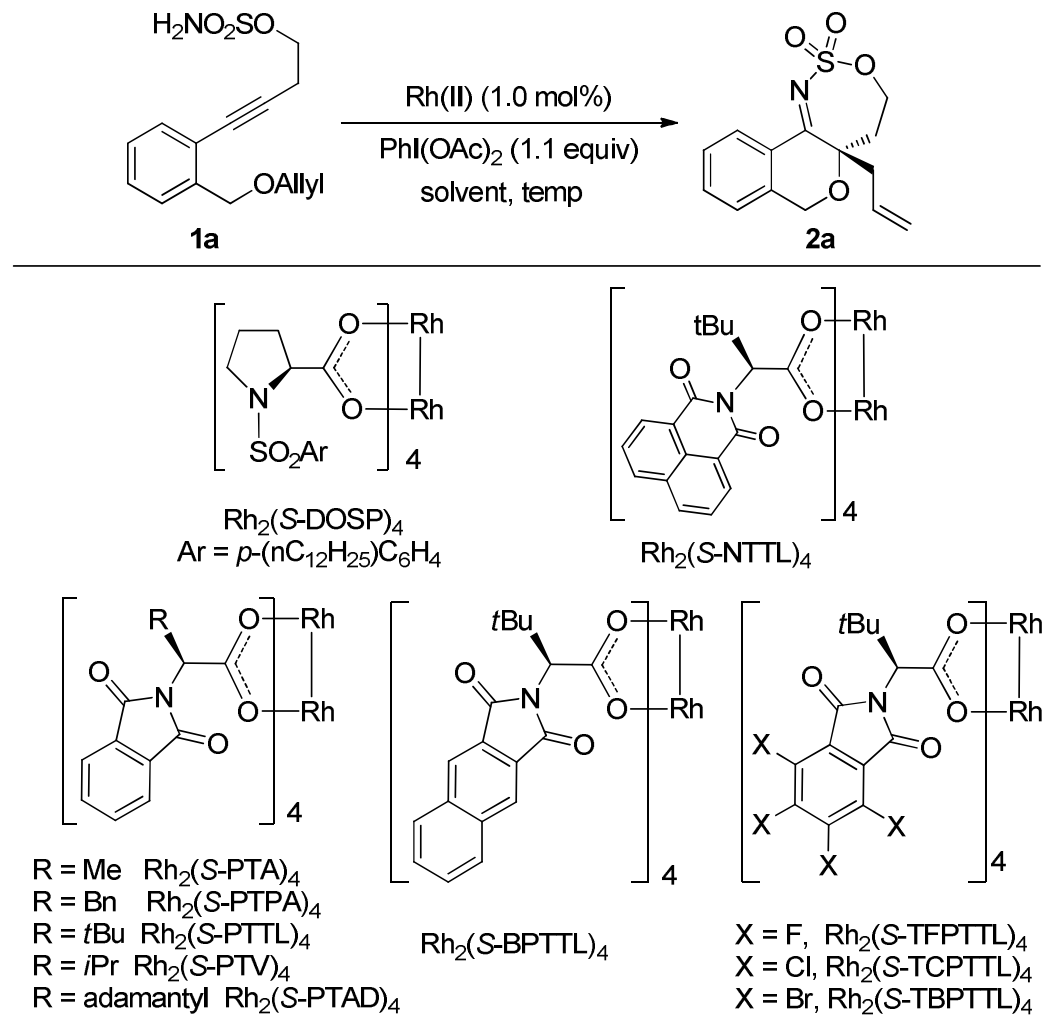

\begin{tabular}{llllll}
\hline entry & $\mathrm{Rh}(\mathrm{II})$ & solvent & $\mathrm{T}\left({ }^{\circ} \mathrm{C}\right)$ & yields $(\%)^{b}$ & ee $(\%)^{c}$ \\
\hline 1 & $\mathrm{Rh}_{2}(\mathrm{esp})_{2}$ & $\mathrm{DCM}$ & $\mathrm{rt}$ & 85 & 0 \\
2 & $\mathrm{Rh}_{2}(\mathrm{~S}-\mathrm{PTTL})_{4}$ & $\mathrm{DCM}$ & $\mathrm{rt}$ & 75 & 26
\end{tabular}




\begin{tabular}{|c|c|c|c|c|c|}
\hline 3 & $\mathrm{Rh}_{2}(S \text {-NTTL })_{4}$ & DCM & $\mathrm{rt}$ & 71 & 40 \\
\hline 4 & $\mathrm{Rh}_{2}(S \text {-TBPTTL })_{4}$ & $\mathrm{DCM}$ & $\mathrm{rt}$ & 69 & 64 \\
\hline 5 & $\mathrm{Rh}_{2}(S \text {-TCPTTL })_{4}$ & DCM & $\mathrm{rt}$ & 61 & 72 \\
\hline 6 & $\mathrm{Rh}_{2}(S \text {-TFPTTL })_{4}$ & $\mathrm{DCM}$ & $\mathrm{rt}$ & 70 & 65 \\
\hline 7 & $\mathrm{Rh}_{2}(S \text {-PTAD })_{4}$ & DCM & $\mathrm{rt}$ & 77 & 36 \\
\hline 8 & $\mathrm{Rh}_{2}(S \text {-PTA })_{4}$ & DCM & $\mathrm{rt}$ & 78 & 20 \\
\hline 9 & $\mathrm{Rh}_{2}(S \text {-PTV })_{4}$ & $\mathrm{DCM}$ & $\mathrm{rt}$ & 81 & 17 \\
\hline 10 & $\mathrm{Rh}_{2}(S \text {-PTPA })_{4}$ & DCM & $\mathrm{rt}$ & 77 & 23 \\
\hline 11 & $\mathrm{Rh}_{2}(S \text {-DOSP })_{4}$ & DCM & $\mathrm{rt}$ & 62 & 30 \\
\hline 12 & $\mathrm{Rh}_{2}(S \text {-BPTTL })_{4}$ & $\mathrm{DCM}$ & $\mathrm{rt}$ & 75 & 21 \\
\hline 13 & $\mathrm{Rh}_{2}(S \text {-TCPTTL })_{4}$ & DCE & $\mathrm{rt}$ & 63 & 62 \\
\hline 14 & $\mathrm{Rh}_{2}(S \text {-TCPTTL })_{4}$ & toluene & $\mathrm{rt}$ & 66 & 83 \\
\hline 15 & $\mathrm{Rh}_{2}(S \text {-TCPTTL })_{4}$ & $\mathrm{C}_{6} \mathrm{H}_{5} \mathrm{Cl}$ & $\mathrm{rt}$ & 64 & 83 \\
\hline 16 & $\mathrm{Rh}_{2}(S \text {-TCPTTL })_{4}$ & $\mathrm{CF}_{3} \mathrm{C}_{6} \mathrm{H}_{5}$ & $\mathrm{rt}$ & 68 & 84 \\
\hline $17^{d}$ & $\mathrm{Rh}_{2}(S \text {-TCPTTL })_{4}$ & TBME & $\mathrm{rt}$ & - & - \\
\hline 18 & $\mathrm{Rh}_{2}(S \text {-TCPTTL })_{4}$ & $\mathrm{CF}_{3} \mathrm{C}_{6} \mathrm{H}_{5}$ & 40 & 65 & 82 \\
\hline $19^{e}$ & $\mathrm{Rh}_{2}(S \text {-TCPTTL })_{4}$ & $\mathrm{CF}_{3} \mathrm{C}_{6} \mathrm{H}_{5}$ & 0 & 67 & 87 \\
\hline $20^{e}$ & $\mathrm{Rh}_{2}(S \text {-TCPTTL })_{4}$ & $\mathrm{CF}_{3} \mathrm{C}_{6} \mathrm{H}_{5}$ & -20 & 60 & 87 \\
\hline $21^{e, f}$ & $\mathrm{Rh}_{2}(S \text {-TCPTTL })_{4}$ & $\mathrm{CF}_{3} \mathrm{C}_{6} \mathrm{H}_{5}$ & 0 & 71 & 90 \\
\hline $22^{f, g}$ & $\mathrm{Rh}_{2}(S \text {-TCPTTL })_{4}$ & $\mathrm{CF}_{3} \mathrm{C}_{6} \mathrm{H}_{5} / \mathrm{TBME}$ & 0 & 77 & 92 \\
\hline
\end{tabular}

${ }^{a}$ Reaction conditions: to Rh-catalyst (1.0 mol\%), and $\mathrm{PhI}(\mathrm{OAc})_{2}(70.8 \mathrm{mg}, 0.22 \mathrm{mmol}, 1.1$ equiv) in corresponding solvent $(0.5 \mathrm{~mL})$, was added $1 \mathrm{a}(59.1 \mathrm{mg}, 0.2 \mathrm{mmol})$ in the same solvent $(1.0 \mathrm{~mL})$ via syringe pump in $1 \mathrm{~h}$ under argon atmosphere, and the reaction mixture was stirred for additional $2 \mathrm{~h}$ under these conditions. ${ }^{b}$ Isolated yields. ${ }^{c}$ Determined by chiral HPLC analysis, see SI for details. ${ }^{d}$ Low conversion $(<5 \%)$ was observed and most of the material 1a was recovered. ${ }^{e}$ The reaction was carried out for $18 \mathrm{~h} .{ }^{f}$ Additive $4 \AA$ MS (100 $\mathrm{mg})$ was used. ${ }^{g}$ The reaction was carried out in mixed solvent $\left(\mathrm{CF}_{3} \mathrm{C}_{6} \mathrm{H}_{5}: \mathrm{TBME}=10: 1\right)$ for 60 h. DCM = dichloromethane. DCE = 1,2-dichloroethane. TBME = tert-butyl methyl ether. 


\section{General Procedure for the Synthesis of Sulfamate Esters 1. ${ }^{1}$}

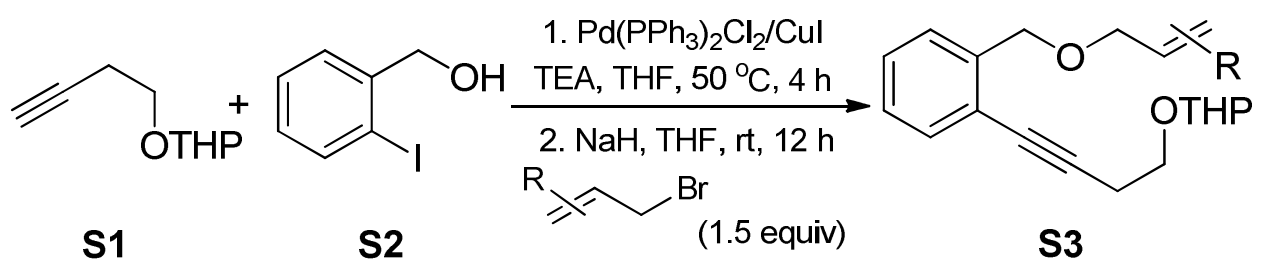

Synthesis of S3: To a 100-mL oven-dried round bottom flask containing a magnetic stirring bar, S1 (1.69 g, $11.0 \mathrm{mmol}, 1.1$ equiv), $\mathrm{Pd}\left(\mathrm{PPh}_{3}\right)_{2} \mathrm{Cl}_{2}(70.2 \mathrm{mg}, 1.0 \mathrm{~mol} \%)$, and CuI (19.0 mg, $1.0 \mathrm{~mol} \%)$ in THF (15 mL), was added (2-iodophenyl)methanol (2.34g, $10.0 \mathrm{mmol}, 1.0$ equiv) and $\mathrm{Et}_{3} \mathrm{~N}(15 \mathrm{~mL})$ under nitrogen atmosphere at room temperature. The resulting mixture was stirred at $50{ }^{\circ} \mathrm{C}$ for $4 \mathrm{~h}$. After the reaction finished, the mixture was filtered by a short silica pad, then the solvent was evaporated under reduced pressure and the residue was purified by flash chromatography on silica gel using petroleum ether and ethyl acetate $(\mathrm{PE} / \mathrm{EA}=5 / 1)$ as the eluent to afford the crude coupling product. The above obtained crude product (1.30 g, $5.0 \mathrm{mmol})$ was dissolved in anhydrous THF (10 mL), NaH (600 mg, 60\%, $10.0 \mathrm{mmol}, 2.0$ equiv) was added to the reaction mixture at room temperature and stirred under this condition for $30 \mathrm{~min}$. Allyl bromide ( $7.5 \mathrm{mmol}, 1.5$ equiv) was then added drop wise. The resulting suspension was stirred overnight at room temperature. Saturated aqueous ammonium chloride solution $(20 \mathrm{~mL})$ was added to quench the reaction, followed by extraction with ethyl acetate $(3 \times 50 \mathrm{~mL})$. The combined organic extract was dried $\left(\mathrm{Na}_{2} \mathrm{SO}_{4}\right)$, filtered and solvent removed in vacuo to give the crude product $\mathbf{S 3}$ as an oil, which was used in the next step without further purification.

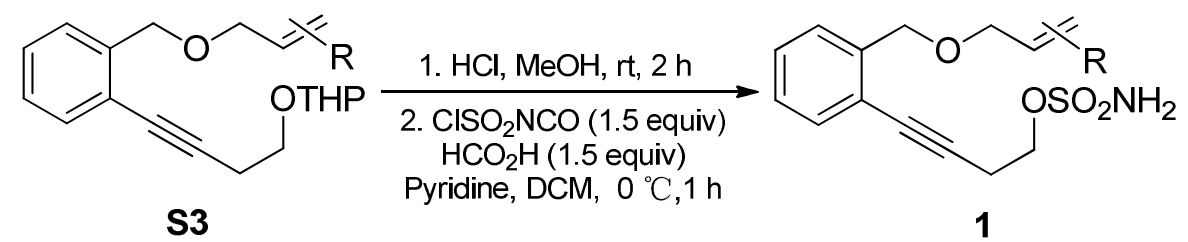

Synthesis of 1: To a 100-mL oven-dried round bottom flask containing a magnetic 
stirring bar, $\mathbf{S 3}$ in $\mathrm{MeOH}(30 \mathrm{~mL})$, was added $\mathrm{HCl}(0.5 \mathrm{~mL}, 3 \mathrm{~N})$ at room temperature, and the reaction mixture was stirred under these conditions for $2 \mathrm{~h}$. Then the most of the solvent was evaporated in vacuo, and water $(30 \mathrm{~mL})$ was added. The reaction mixture was extracted with ethyl acetate $(3 \times 50 \mathrm{~mL})$. The combined organic layer was washed with brine $(2 \times 50 \mathrm{~mL})$, dried over anhydrous $\mathrm{Na}_{2} \mathrm{SO}_{4}$, filtered and solvent removed in vacuo to give the crude product as an oil, which was used in the next step without further purification. To a 50-mL oven-dried round bottom flask equipped with stirring bar and $\mathrm{ClSO}_{2} \mathrm{NCO}(0.65 \mathrm{~mL}, 7.5 \mathrm{mmol}, 1.5$ equiv), was added formic acid (0.29 mL, $7.5 \mathrm{mmol}, 1.5$ equiv) drop wise under an argon atmosphere at $0{ }^{\circ} \mathrm{C}$. The resulting white solid was dissolved in $\mathrm{CH}_{2} \mathrm{Cl}_{2}(5 \mathrm{~mL})$. The solution was warmed to $25{ }^{\circ} \mathrm{C}$ and allowed to stir overnight. To this reaction mixture, the solution of above obtained crude product and pyridine $\left(0.6 \mathrm{~mL}, 7.5 \mathrm{mmol}, 1.5\right.$ equiv) in $\mathrm{CH}_{2} \mathrm{Cl}_{2}$ (4 M) was added drop wise at $0{ }^{\circ} \mathrm{C}$. The reaction mixture was warmed to $25^{\circ} \mathrm{C}$ and stirred until complete consumption of starting material (15 $\min \sim 1 \mathrm{~h}$, monitored by thin layer chromatography). Then the reaction was quenched by addition of $\mathrm{H}_{2} \mathrm{O}(10 \mathrm{~mL})$ and extracted with EtOAc $(3 \times 20 \mathrm{~mL})$. The combined organic extract was washed with brine $(50 \mathrm{~mL})$, dried over anhydrous $\mathrm{Na}_{2} \mathrm{SO}_{4}$, filtered, and concentrated under reduced pressure. Purification by flash chromatography on silica gel using petroleum ether and ethyl acetate $(\mathrm{PE} / \mathrm{EA}=3 / 1$ to $1 / 1)$ as the eluent to afford pure sulfamate ester $\mathbf{1}$ in high yields.

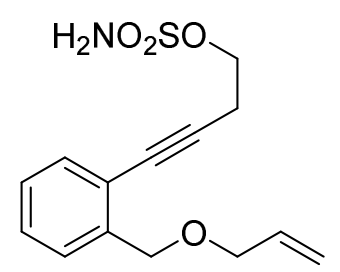

4-\{2-[(Allyloxy)methyl]phenyl\}but-3-yn-1-yl sulfamate (1a). ${ }^{1 a}$ Colorless oil. ${ }^{1} \mathrm{H}$ NMR (400 MHz, $\left.\mathrm{CDCl}_{3}\right)(\delta, \mathrm{ppm}) 7.44(\mathrm{~d}, J=7.2 \mathrm{~Hz}, 1 \mathrm{H}), 7.40$ (d, $\left.J=7.6 \mathrm{~Hz}, 1 \mathrm{H}\right)$, $7.32(\mathrm{t}, J=7.6 \mathrm{~Hz}, 1 \mathrm{H}), 7.27$ (t, $J=7.6 \mathrm{~Hz}, 1 \mathrm{H}), 5.96(\mathrm{~m}, 1 \mathrm{H}), 5.34$ (d, $J=17.2,1 \mathrm{H})$, $5.24(\mathrm{~d}, J=10.5 \mathrm{~Hz}, 1 \mathrm{H}), 5.14(\mathrm{~s}, 2 \mathrm{H}), 4.67(\mathrm{~s}, 2 \mathrm{H}), 4.38$ (t, $J=6.2 \mathrm{~Hz}, 2 \mathrm{H}), 4.06$ (d, 
$J=5.7 \mathrm{~Hz}, 2 \mathrm{H}), 2.92(\mathrm{t}, J=6.2 \mathrm{~Hz}, 2 \mathrm{H}) ;{ }^{13} \mathrm{C} \mathrm{NMR}\left(100 \mathrm{MHz}, \mathrm{CDCl}_{3}\right)(\delta, \mathrm{ppm}) 139.6$, $134.6,132.6,129.0,128.5,128.0,122.5,118.0,89.2,80.7,71.3,70.6,68.7,21.0$.

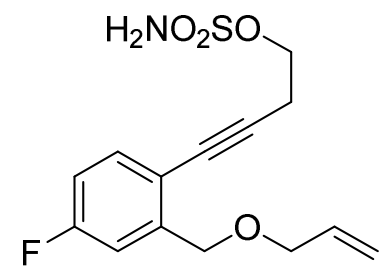

4-\{2-[(Allyloxy)methyl]-4-fluorophenyl\}but-3-yn-1-yl sulfamate (1b). Colorless oil. ${ }^{1} \mathrm{H}$ NMR $\left(400 \mathrm{MHz}, \mathrm{CDCl}_{3}\right)(\delta, \mathrm{ppm}) 7.37$ (dd, $\left.J=8.5,5.6 \mathrm{~Hz}, 1 \mathrm{H}\right), 7.16(\mathrm{dd}, J=9.5$, $2.5 \mathrm{~Hz}, 1 \mathrm{H}), 6.93-6.89(\mathrm{~m}, 1 \mathrm{H}), 6.00-5.91(\mathrm{~m}, 1 \mathrm{H}), 5.49(\mathrm{~s}, 2 \mathrm{H}), 5.33(\mathrm{dd}, J=17.2$, $1.5 \mathrm{~Hz}, 1 \mathrm{H}), 5.23(\mathrm{dd}, J=10.4,1.0 \mathrm{~Hz}, 1 \mathrm{H}), 4.63(\mathrm{~s}, 2 \mathrm{H}), 4.31$ (t, $J=6.6 \mathrm{~Hz}, 2 \mathrm{H})$, $4.08(\mathrm{~d}, J=5.7 \mathrm{~Hz}, 2 \mathrm{H}), 2.86(\mathrm{t}, J=6.6 \mathrm{~Hz}, 2 \mathrm{H}) ;{ }^{13} \mathrm{C} \mathrm{NMR}\left(100 \mathrm{MHz}, \mathrm{CDCl}_{3}\right)(\delta$, ppm) $162.5(\mathrm{~d}, J=249.3 \mathrm{~Hz}), 142.8(\mathrm{~d}, J=7.6 \mathrm{~Hz}), 134.3,134.0(\mathrm{~d}, J=8.2 \mathrm{~Hz})$, 117.8, $117.4(\mathrm{~d}, J=3.5 \mathrm{~Hz}), 114.8(\mathrm{~d}, J=23.1 \mathrm{~Hz}), 114.6(\mathrm{~d}, J=22.2 \mathrm{~Hz}), 88.8,79.3$, 71.5, 69.7, 68.5, 20.4; ${ }^{19} \mathrm{~F}$ NMR (376 MHz, $\left.\mathrm{CDCl}_{3}\right)(\delta$, ppm) -110.56; HRMS (TOF MS ESI ${ }^{+}$) calculated for $\mathrm{C}_{14} \mathrm{H}_{16} \mathrm{FNO}_{4} \mathrm{SNa}[\mathrm{M}+\mathrm{Na}]^{+}$: 336.0676, found 336.0664.

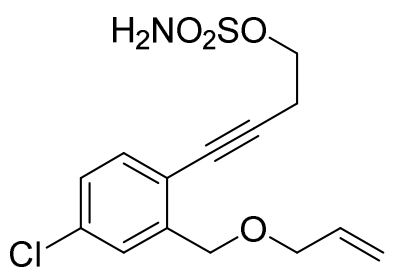

4-\{2-[(Allyloxy)methyl]-4-chlorophenyl\}but-3-yn-1-yl sulfamate (1c). Colorless oil. ${ }^{1} \mathrm{H}$ NMR $\left(400 \mathrm{MHz}, \mathrm{CDCl}_{3}\right)(\delta, \mathrm{ppm}) 7.42(\mathrm{~d}, J=2.0 \mathrm{~Hz}, 1 \mathrm{H}), 7.32(\mathrm{~d}, J=8.2 \mathrm{~Hz}$, 1H), $7.21-7.18(\mathrm{~m}, 1 \mathrm{H}), 6.00-5.89(\mathrm{~m}, 1 \mathrm{H}), 5.35-5.22(\mathrm{comp}, 4 \mathrm{H}), 4.61(\mathrm{~s}, 2 \mathrm{H})$, $4.33(\mathrm{t}, J=6.5 \mathrm{~Hz}, 2 \mathrm{H}), 4.08-4.06(\mathrm{~m}, 2 \mathrm{H}), 2.88(\mathrm{t}, J=6.5 \mathrm{~Hz}, 2 \mathrm{H}) ;{ }^{13} \mathrm{C}$ NMR $(100$ $\left.\mathrm{MHz}, \mathrm{CDCl}_{3}\right)(\delta, \mathrm{ppm}) 141.8,134.4,134.3,133.5,130.5,128.2,127.8,117.9,90.1$, 79.5, 71.6, 69.8, 68.4, 20.6; HRMS (TOF MS ESI ${ }^{+}$) calculated for $\mathrm{C}_{14} \mathrm{H}_{16} \mathrm{ClNO}_{4} \mathrm{SNa}$ $[\mathrm{M}+\mathrm{Na}]^{+}:$352.0381, found 352.0379 . 


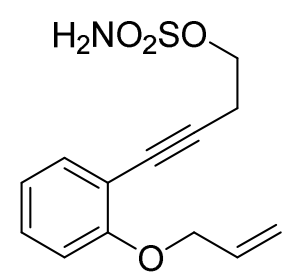

4-[2-(Allyloxy)phenyl]but-3-yn-1-yl sulfamate (1d). White solid, $\mathrm{mp}=85-86{ }^{\circ} \mathrm{C}$; ${ }^{1} \mathrm{H}$ NMR $\left(400 \mathrm{MHz}, \mathrm{CDCl}_{3}\right)(\delta, \mathrm{ppm}) 7.24-7.22(\mathrm{~m}, 1 \mathrm{H}), 7.12-7.07$ (m, 1H), 6.77 - $6.70(\mathrm{~m}, 2 \mathrm{H}), 5.97-5.87(\mathrm{~m}, 1 \mathrm{H}), 5.32-5.27$ (m, 1H), $5.18-5.15(\mathrm{~m}, 3 \mathrm{H}), 4.47$ $4.45(\mathrm{~m}, 2 \mathrm{H}), 4.20(\mathrm{t}, J=6.5 \mathrm{~Hz}, 2 \mathrm{H}), 2.73(\mathrm{t}, J=6.5 \mathrm{~Hz}, 2 \mathrm{H}) ;{ }^{13} \mathrm{C} \mathrm{NMR}(100 \mathrm{MHz}$, $\left.\mathrm{CDCl}_{3}\right)(\delta, \mathrm{ppm}) 158.9,133.7,132.9,129.6,121.0,118.1,112.6,112.5,89.0,79.0$, 69.5, 68.8, 20.7; HRMS (TOF MS ESI ${ }^{+}$) calculated for $\mathrm{C}_{13} \mathrm{H}_{15} \mathrm{NO}_{4} \mathrm{SNa}[\mathrm{M}+\mathrm{Na}]^{+}$: 304.0614 , found 304.0625 .<smiles>C=C(COCc1ccccc1C#CCCOS(N)(=O)=O)c1ccccc1</smiles>

4-\{2-[((2-Phenylallyl)oxy)methyl]phenyl\}but-3-yn-1-yl sulfamate (1e). Yellow oil. ${ }^{1} \mathrm{H}$ NMR $\left(400 \mathrm{MHz}, \mathrm{CDCl}_{3}\right)(\delta, \mathrm{ppm}) 7.49-7.46(\mathrm{~m}, 2 \mathrm{H}), 7.43(\mathrm{~m}, 1 \mathrm{H}), 7.39-7.29$ (comp, 5H), $7.28-7.22(\mathrm{~m}, 1 \mathrm{H}), 5.58$ (d, $J=0.9 \mathrm{~Hz}, 1 \mathrm{H}), 5.40(\mathrm{~d}, J=1.2 \mathrm{~Hz}, 1 \mathrm{H})$, $5.19(\mathrm{~s}, 2 \mathrm{H}), 4.72(\mathrm{~s}, 2 \mathrm{H}), 4.44(\mathrm{~d}, J=0.7 \mathrm{~Hz}, 2 \mathrm{H}), 4.26(\mathrm{t}, J=6.5 \mathrm{~Hz}, 2 \mathrm{H}), 2.83(\mathrm{t}, J$ $=6.5 \mathrm{~Hz}, 2 \mathrm{H}) ;{ }^{13} \mathrm{C} \mathrm{NMR}\left(100 \mathrm{MHz}, \mathrm{CDCl}_{3}\right)(\delta, \mathrm{ppm}) 144.0,139.5,138.7,132.4$, $128.7,128.5,128.3,127.9,127.8,126.3,122.4,115.2,89.1,80.5,72.0,70.3,68.4$, 20.5; HRMS (TOF MS ESI ${ }^{+}$) calculated for $\mathrm{C}_{20} \mathrm{H}_{21} \mathrm{NO}_{4} \mathrm{SNa}[\mathrm{M}+\mathrm{Na}]^{+}: 394.1083$, found 394.1074. 
<smiles>C=C(COCc1ccccc1C#CCCOS(N)(=O)=O)c1ccc(C(F)(F)F)cc1</smiles>

\section{4-\{2-[((2-(4-(Trifluoromethyl)phenyl)allyl)oxy)methyl]phenyl\}but-3-yn-1-yl}

sulfamate (1f). Yellow oil. ${ }^{1} \mathrm{H}$ NMR $\left(400 \mathrm{MHz}, \mathrm{CDCl}_{3}\right)(\delta$, ppm) 7.59 - 7.54 (comp, 4H), $7.43-7.41(\mathrm{~m}, 1 \mathrm{H}), 7.35-7.33(\mathrm{~m}, 1 \mathrm{H}), 7.31-7.21(\mathrm{~m}, 2 \mathrm{H}), 5.64(\mathrm{~s}, 1 \mathrm{H}), 5.50$ $(\mathrm{d}, J=0.9 \mathrm{~Hz}, 1 \mathrm{H}), 5.32(\mathrm{~s}, 2 \mathrm{H}), 4.70(\mathrm{~s}, 2 \mathrm{H}), 4.44(\mathrm{~d}, J=0.5 \mathrm{~Hz}, 2 \mathrm{H}), 4.28(\mathrm{t}, J=6.5$ $\mathrm{Hz}, 2 \mathrm{H}), 2.83(\mathrm{t}, J=6.5 \mathrm{~Hz}, 2 \mathrm{H}) ;{ }^{13} \mathrm{C} \mathrm{NMR}\left(100 \mathrm{MHz}, \mathrm{CDCl}_{3}\right)(\delta, \mathrm{ppm}) 143.1,142.2$, 139.3, 132.5, 129.7 (q, $J=32.4 \mathrm{~Hz}), 128.6,128.4,127.8,126.6,125.4$ (q, $J=3.8 \mathrm{~Hz})$, $124.3(\mathrm{q}, J=272.0 \mathrm{~Hz}), 122.3,117.3,89.2,80.4,71.9,70.4,68.5,20.5 ;{ }^{19} \mathrm{~F}$ NMR $\left(376 \mathrm{MHz}, \mathrm{CDCl}_{3}\right)(\delta, \mathrm{ppm})-62.46$; HRMS (TOF MS $\mathrm{ESI}^{+}$) calculated for $\mathrm{C}_{21} \mathrm{H}_{20} \mathrm{~F}_{3} \mathrm{NO}_{4} \mathrm{SNa}[\mathrm{M}+\mathrm{Na}]^{+}:$462.0957, found 462.0964 .

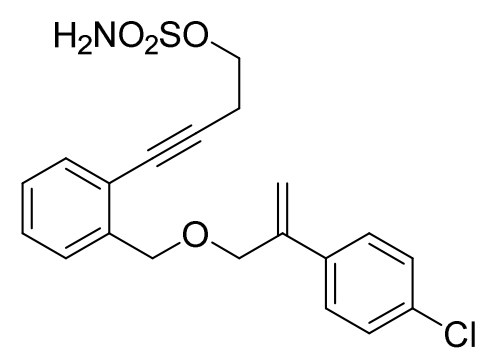

4-\{2-[((2-(4-Chlorophenyl)allyl)oxy)methyl]phenyl\}but-3-yn-1-yl sulfamate (1g). Yellow oil. ${ }^{1} \mathrm{H}$ NMR $\left(400 \mathrm{MHz}, \mathrm{CDCl}_{3}\right)(\delta, \mathrm{ppm}) 7.44-7.38$ (comp, 3H), 7.35 - 7.33 (m, 1H), $7.30-7.22$ (comp, 4H), $5.56(\mathrm{~s}, 1 \mathrm{H}), 5.40$ (d, $J=0.8 \mathrm{~Hz}, 1 \mathrm{H}), 5.27$ (s, 2H), $4.68(\mathrm{~s}, 2 \mathrm{H}), 4.40(\mathrm{~s}, 2 \mathrm{H}), 4.28(\mathrm{t}, J=6.5 \mathrm{~Hz}, 2 \mathrm{H}), 2.84(\mathrm{t}, J=6.5 \mathrm{~Hz}, 2 \mathrm{H}) ;{ }^{13} \mathrm{C} \mathrm{NMR}$ $\left(100 \mathrm{MHz}, \mathrm{CDCl}_{3}\right)(\delta, \mathrm{ppm}) 143.0,139.3,137.0,133.7,132.5,128.9,128.6(\mathrm{t}, J=$ $12.8 \mathrm{~Hz}), 128.2,127.8,127.6,122.3,115.8,89.1,80.5,72.0,70.3,68.5,20.5$; HRMS (TOF MS ESI ${ }^{+}$) calculated for $\mathrm{C}_{20} \mathrm{H}_{20} \mathrm{ClNO}_{4} \mathrm{SNa}[\mathrm{M}+\mathrm{Na}]^{+}:$428.0694, found 428.0697. 
<smiles>C=C(COCc1ccccc1C#CCCOS(N)(=O)=O)c1ccc(Br)cc1</smiles>

4-\{2-[((2-(4-Bromophenyl)allyl)oxy)methyl]phenyl\}but-3-yn-1-yl sulfamate (1h). Yellow oil. ${ }^{1} \mathrm{H}$ NMR (400 MHz, $\left.\mathrm{CDCl}_{3}\right)(\delta$, ppm) $\delta 7.45-7.40$ (comp, 3H), $7.36-$ 7.31 (comp, 3H), 7.30 - 7.27 (m, 1H), 7.25 - 7.21 (m, 1H), 5.56 (s, 1H), 5.41 (comp, $3 \mathrm{H}), 4.68(\mathrm{~s}, 2 \mathrm{H}), 4.40(\mathrm{~s}, 2 \mathrm{H}), 4.27(\mathrm{t}, J=6.6 \mathrm{~Hz}, 2 \mathrm{H}), 2.83(\mathrm{t}, J=6.5 \mathrm{~Hz}, 2 \mathrm{H}) ;{ }^{13} \mathrm{C}$ NMR (100 MHz, $\left.\mathrm{CDCl}_{3}\right)(\delta, \mathrm{ppm}) 142.9,139.3,137.4,132.4,131.8,131.5,128.5$, 128.4, 127.9, 127.7, 122.2, 115.8, 89.2, 80.4, 71.9, 70.2, 68.4, 20.4; HRMS (TOF MS $\mathrm{ESI}^{+}$) calculated for $\mathrm{C}_{20} \mathrm{H}_{20} \mathrm{BrNO}_{4} \mathrm{SNa}[\mathrm{M}+\mathrm{Na}]^{+}$: 472.0189, found 472.0197.

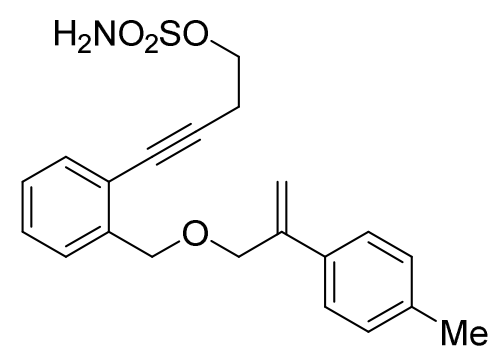

4-\{2-[((2-(p-Tolyl)allyl)oxy)methyl]phenyl\}but-3-yn-1-yl sulfamate (1i). Yellow oil. ${ }^{1} \mathrm{H}$ NMR $\left(400 \mathrm{MHz}, \mathrm{CDCl}_{3}\right)(\delta, \mathrm{ppm}) 7.45$ - 7.43 (m, 1H), $7.41-7.37$ (comp , 3H), $7.32-7.28(\mathrm{~m}, 1 \mathrm{H}), 7.27-7.23(\mathrm{~m}, 1 \mathrm{H}), 7.16(\mathrm{~d}, J=8.0 \mathrm{~Hz}, 2 \mathrm{H}), 5.55(\mathrm{~s}, 1 \mathrm{H})$, $5.36(\mathrm{~d}, J=0.8 \mathrm{~Hz}, 1 \mathrm{H}), 5.23(\mathrm{~s}, 2 \mathrm{H}), 4.72(\mathrm{~s}, 2 \mathrm{H}), 4.43(\mathrm{~s}, 2 \mathrm{H}), 4.27$ (t, $J=6.5 \mathrm{~Hz}$, $2 \mathrm{H}), 2.84(\mathrm{t}, J=6.5 \mathrm{~Hz}, 2 \mathrm{H}), 2.35(\mathrm{~s}, 3 \mathrm{H}) ;{ }^{13} \mathrm{C} \mathrm{NMR}\left(100 \mathrm{MHz}, \mathrm{CDCl}_{3}\right)(\delta, \mathrm{ppm})$ $143.8,139.5,137.8,135.7,132.4,129.2,128.8,128.4,127.7,126.1,122.4,114.3$, 89.2, 80.5, 72.1, 70.3, 68.4, 21.2, 20.5; HRMS (TOF MS ESI ${ }^{+}$) calculated for $\mathrm{C}_{21} \mathrm{H}_{23} \mathrm{NO}_{4} \mathrm{SNa}[\mathrm{M}+\mathrm{Na}]^{+}:$408.1240, found 408.1240. 
<smiles>C=C(COCc1ccccc1C#CCCOS(N)(=O)=O)c1cccc(OC)c1</smiles>

4-\{2-[((2-(3-Methoxyphenyl)allyl)oxy)methyl]phenyl\}but-3-yn-1-yl sulfamate (1j). Yellow oil. ${ }^{1} \mathrm{H}$ NMR (400 MHz, $\left.\mathrm{CDCl}_{3}\right)(\delta, \mathrm{ppm}) 7.42$ - $7.38(\mathrm{~m}, 2 \mathrm{H}), 7.31-7.23$ (comp, 3H), 7.06 (d, J=7.8 Hz, 1H), $7.02-7.01(\mathrm{~m}, 1 \mathrm{H}), 6.85-6.83(\mathrm{~m}, 1 \mathrm{H}), 5.57$ (s, 1H), 5.39 (d, J=0.9 Hz, 1H), $5.29(\mathrm{~s}, 2 \mathrm{H}), 4.71(\mathrm{~s}, 2 \mathrm{H}), 4.41(\mathrm{~s}, 2 \mathrm{H}), 4.26$ (t, $J=$ $6.5 \mathrm{~Hz}, 2 \mathrm{H}), 3.80(\mathrm{~s}, 3 \mathrm{H}), 2.83(\mathrm{t}, J=6.5 \mathrm{~Hz}, 2 \mathrm{H}) ;{ }^{13} \mathrm{C} \mathrm{NMR}\left(100 \mathrm{MHz}, \mathrm{CDCl}_{3}\right)(\delta$, ppm) 159.6, 144.0, 140.3, 139.5, 132.4, 129.5, 128.7, 128.4, 127.8, 122.4, 118.8, 115.5, 113.3, 112.1, 89.2, 80.5, 72.1, 70.3, 68.4, 55.4, 20.5; HRMS (TOF MS ESI ${ }^{+}$) calculated for $\mathrm{C}_{21} \mathrm{H}_{23} \mathrm{NO}_{5} \mathrm{SNa}[\mathrm{M}+\mathrm{Na}]^{+}$: 424.1189, found 424.1172.

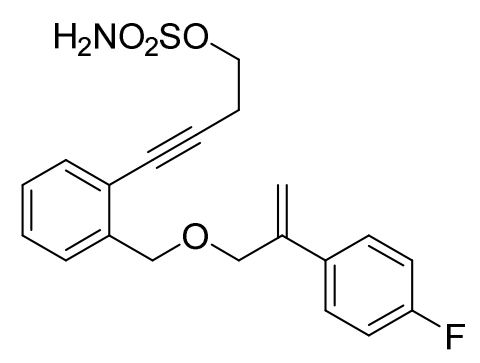

4-\{2-[((2-(4-Fluorophenyl)allyl)oxy)methyl]phenyl\}but-3-yn-1-yl sulfamate (1k). Yellow oil. ${ }^{1} \mathrm{H}$ NMR $\left(400 \mathrm{MHz}, \mathrm{CDCl}_{3}\right)(\delta, \mathrm{ppm}) 7.45$ - $7.41(\mathrm{~m}, 2 \mathrm{H}), 7.39$ - $7.34(\mathrm{~m}$, 2H), $7.31-7.22(\mathrm{~m}, 2 \mathrm{H}), 7.09-6.99(\mathrm{~m}, 2 \mathrm{H}), 5.51(\mathrm{~s}, 1 \mathrm{H}), 5.36(\mathrm{~s}, 1 \mathrm{H}), 5.23(\mathrm{~s}, 2 \mathrm{H})$, $4.69(\mathrm{~s}, 2 \mathrm{H}), 4.40(\mathrm{~d}, J=0.7 \mathrm{~Hz}, 2 \mathrm{H}), 4.29$ (t, $J=6.5 \mathrm{~Hz}, 2 \mathrm{H}), 2.85$ (t, $J=6.5 \mathrm{~Hz}$, $2 \mathrm{H}) ;{ }^{13} \mathrm{C}$ NMR $\left(100 \mathrm{MHz}, \mathrm{CDCl}_{3}\right)(\delta, \mathrm{ppm}) 162.6(\mathrm{~d}, J=247.0 \mathrm{~Hz}), 143.1,139.4$, $134.7(\mathrm{~d}, J=3.2 \mathrm{~Hz}), 132.5,128.6$ (d, $J=31.7 \mathrm{~Hz}), 128.6$ (d, $J=8.1 \mathrm{~Hz}), 128.0$ (d, $J$ $=8.0 \mathrm{~Hz}), 127.8,122.4,115.6(\mathrm{~d}, J=21.3 \mathrm{~Hz}), 115.3(\mathrm{~d}, J=21.3 \mathrm{~Hz}), 89.1,80.5$, 72.2, 70.3, 68.5, 20.6; ${ }^{19} \mathrm{~F}$ NMR $\left(376 \mathrm{MHz} \mathrm{CDCl}_{3}\right)(\delta, \mathrm{ppm})-114.38$; HRMS (TOF MS ESI ${ }^{+}$) calculated for $\mathrm{C}_{20} \mathrm{H}_{20} \mathrm{FNO}_{4} \mathrm{SNa}[\mathrm{M}+\mathrm{Na}]^{+}$: 412.0989, found 412.0999. 


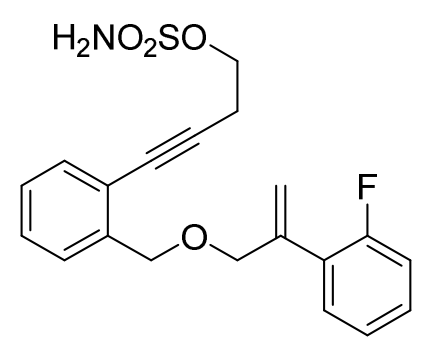

4-\{2-[((2-(2-Fluorophenyl)allyl)oxy)methyl]phenyl\}but-3-yn-1-yl sulfamate (11). Yellow oil. ${ }^{1} \mathrm{H}$ NMR (400 MHz, $\left.\mathrm{CDCl}_{3}\right)(\delta$, ppm) $7.40(\mathrm{dd}, J=7.4,1.3 \mathrm{~Hz}, 1 \mathrm{H}), 7.36$ - $7.31(\mathrm{~m}, 2 \mathrm{H}), 7.29-7.19(\mathrm{comp}, 3 \mathrm{H}), 7.11(\mathrm{~m}, 1 \mathrm{H}), 7.04(\mathrm{~m}, 1 \mathrm{H}), 5.56(\mathrm{~d}, J=1.0$ Hz, 1H), 5.47 (s, 1H), 5.28 (s, 2H), 4.68 (s, 2H), 4.40 (s, 2H), 4.29 (t, J=6.6 Hz, 2H), $2.85(\mathrm{t}, J=6.5 \mathrm{~Hz}, 2 \mathrm{H}) ;{ }^{13} \mathrm{C} \mathrm{NMR}\left(100 \mathrm{MHz}, \mathrm{CDCl}_{3}\right)(\delta, \mathrm{ppm}) 160.1(\mathrm{~d}, J=247.7 \mathrm{~Hz})$, 141.0, 139.5, 132.4, 130.2 (d, $J=4.2 \mathrm{~Hz}), 129.4$ (d, $J=8.4 \mathrm{~Hz}), 128.4$ (d, $J=19.0$ $\mathrm{Hz}), 127.7,127.1$ (d, $J=14.0 \mathrm{~Hz}), 124.2(\mathrm{~d}, J=3.5 \mathrm{~Hz}), 122.2,118.2(\mathrm{~d}, J=3.4 \mathrm{~Hz})$, 116.0, 115.7, 89.1, 80.4, $72.4(\mathrm{~d}, J=4.0 \mathrm{~Hz}), 70.3,68.4,20.5 ;{ }^{19} \mathrm{~F}$ NMR (376 MHz, $\left.\mathrm{CDCl}_{3}\right)(\delta, \mathrm{ppm})$-114.81; HRMS (TOF MS ESI ${ }^{+}$) calculated for $\mathrm{C}_{20} \mathrm{H}_{20} \mathrm{FNO}_{4} \mathrm{SNa}$ $[\mathrm{M}+\mathrm{Na}]^{+}:$412.0989, found 412.0996 .<smiles>C=C(COCc1ccccc1C#CCCOS(N)(=O)=O)c1cccc(F)c1</smiles>

\section{4-\{2-[((2-(3-Fluorophenyl)allyl)oxy)methyl]phenyl\}but-3-yn-1-yl sulfamate (1 m).}

Yellow oil. ${ }^{1} \mathrm{H}$ NMR $\left(400 \mathrm{MHz}, \mathrm{CDCl}_{3}\right)(\delta, \mathrm{ppm}) 7.42(\mathrm{dd}, J=7.5,1.3 \mathrm{~Hz}, 1 \mathrm{H}), 7.36$ (d, $J=7.4 \mathrm{~Hz}, 1 \mathrm{H}), 7.31-7.22$ (comp, 4H), $7.19-7.15(\mathrm{~m}, 1 \mathrm{H}), 7.00-6.95(\mathrm{~m}, 1 \mathrm{H})$, 5.59 (s, 1H), 5.43 (d, $J=0.8 \mathrm{~Hz}, 1 \mathrm{H}), 5.24$ (s, 2H), 4.70 (s, 2H), 4.40 (d, $J=0.7 \mathrm{~Hz}$, $2 \mathrm{H}), 4.29(\mathrm{t}, J=6.5 \mathrm{~Hz}, 2 \mathrm{H}), 2.85(\mathrm{t}, J=6.5 \mathrm{~Hz}, 2 \mathrm{H}) ;{ }^{13} \mathrm{C} \mathrm{NMR}\left(100 \mathrm{MHz}, \mathrm{CDCl}_{3}\right)(\delta$, ppm) $162.9(\mathrm{~d}, J=245.0 \mathrm{~Hz}), 143.1(\mathrm{~d}, J=2.2 \mathrm{~Hz}), 141.0(\mathrm{~d}, J=7.6 \mathrm{~Hz}), 139.4$, 132.5, 129.9 (d, $J=8.4 \mathrm{~Hz}), 128.7,128.4,127.8,122.4,121.9$ (d, $J=2.8 \mathrm{~Hz}), 116.4$, $114.7(\mathrm{~d}, J=21.2 \mathrm{~Hz}), 113.2(\mathrm{~d}, J=22.1 \mathrm{~Hz}), 89.2,80.5,72.0,70.4,68.5,20.5 ;{ }^{19} \mathrm{~F}$ 
NMR (376 MHz, $\left.\mathrm{CDCl}_{3}\right)(\delta, \mathrm{ppm})$-113.30; HRMS (TOF MS ESI ${ }^{+}$) calculated for $\mathrm{C}_{20} \mathrm{H}_{20} \mathrm{FNO}_{4} \mathrm{SNa}[\mathrm{M}+\mathrm{Na}]^{+}:$412.0989, found 412.1002.

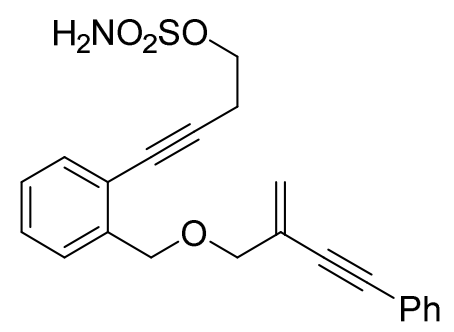

\section{4-\{2-[((2-Methylene-4-phenylbut-3-yn-1-yl)oxy)methyl]phenyl\}but-3-yn-1-yl}

sulfamate (1n). Yellow oil. ${ }^{1} \mathrm{H}$ NMR $\left(400 \mathrm{MHz}, \mathrm{CDCl}_{3}\right)(\delta$, ppm) 7.48 - 7.41 (comp, 4H), $7.32-7.28$ (comp, 4H), $7.25-7.21(\mathrm{~m}, 1 \mathrm{H}), 5.65$ (s, 2H), 5.34 (s, 2H), 4.74 (s, 2H), $4.31(\mathrm{t}, J=6.6 \mathrm{~Hz}, 2 \mathrm{H}), 4.15(\mathrm{~s}, 2 \mathrm{H}), 2.86(\mathrm{t}, J=6.6 \mathrm{~Hz}, 2 \mathrm{H}) ;{ }^{13} \mathrm{C}$ NMR $(100$ $\left.\mathrm{MHz}, \mathrm{CDCl}_{3}\right)(\delta, \mathrm{ppm}) 139.5,132.4,131.7,128.6,128.5,128.4,128.4,128.3,127.7$, 122.9, 122.7, 122.2, 90.5, 89.2, 87.7, 80.4, 72.1, 70.4, 68.4, 20.6; HRMS (TOF MS $\mathrm{ESI}^{+}$) calculated for $\mathrm{C}_{22} \mathrm{H}_{21} \mathrm{NO}_{4} \mathrm{SNa}[\mathrm{M}+\mathrm{Na}]^{+}:$418.1083, found 418.1063.

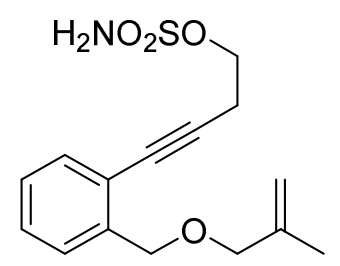

4-\{2-[((2-Methylallyl)oxy)methyl]phenyl\}but-3-yn-1-yl sulfamate (10). Yellow oil. ${ }^{1} \mathrm{H}$ NMR (400 MHz, $\left.\mathrm{CDCl}_{3}\right)(\delta, \mathrm{ppm}) 7.43$ - 7.40 (m, 2H), 7.32 - 7.28 (m, 1H), 7.25 $-7.21(\mathrm{~m}, 1 \mathrm{H}), 5.46(\mathrm{~s}, 2 \mathrm{H}), 5.02(\mathrm{~s}, 1 \mathrm{H}), 4.94(\mathrm{~s}, 1 \mathrm{H}), 4.63(\mathrm{~s}, 2 \mathrm{H}), 4.32(\mathrm{t}, J=6.5$ $\mathrm{Hz}, 2 \mathrm{H}), 3.96(\mathrm{~s}, 2 \mathrm{H}), 2.87(\mathrm{t}, J=6.5 \mathrm{~Hz}, 2 \mathrm{H}), 1.76(\mathrm{~s}, 3 \mathrm{H}) ;{ }^{13} \mathrm{C} \mathrm{NMR}(100 \mathrm{MHz}$, $\left.\mathrm{CDCl}_{3}\right)(\delta, \mathrm{ppm}) 142.1,139.7,132.4,128.4,128.4,127.6,122.1,112.8,89.0,80.5$, 74.1, 70.0, 68.4, 20.5, 19.6; HRMS (TOF MS ESI ${ }^{+}$) calculated for $\mathrm{C}_{15} \mathrm{H}_{19} \mathrm{NO}_{4} \mathrm{SNa}$ $[\mathrm{M}+\mathrm{Na}]^{+}:$332.0927, found 332.0915. 


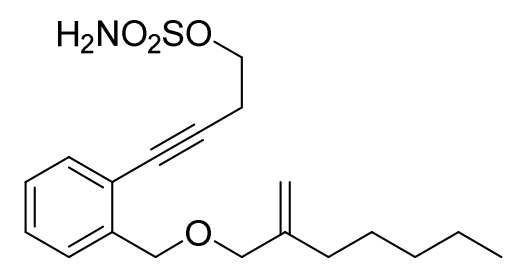

4-\{2-[((2-Methyleneheptyl)oxy)methyl]phenyl\}but-3-yn-1-yl sulfamate (1p). Yellow oil. ${ }^{1} \mathrm{H}$ NMR $\left(400 \mathrm{MHz}, \mathrm{CDCl}_{3}\right)(\delta, \mathrm{ppm}) 7.41(\mathrm{~d}, J=7.7 \mathrm{~Hz}, 2 \mathrm{H}), 7.32-$ $7.28(\mathrm{~m}, 1 \mathrm{H}), 7.25-7.21(\mathrm{~m}, 1 \mathrm{H}), 5.42(\mathrm{~s}, 2 \mathrm{H}), 5.06(\mathrm{~s}, 1 \mathrm{H}), 4.94(\mathrm{~s}, 1 \mathrm{H}), 4.63(\mathrm{~s}$, $2 \mathrm{H}), 4.32(\mathrm{t}, J=6.6 \mathrm{~Hz}, 2 \mathrm{H}), 3.98(\mathrm{~s}, 2 \mathrm{H}), 2.88(\mathrm{t}, J=6.6 \mathrm{~Hz}, 2 \mathrm{H}), 2.08-2.04(\mathrm{~m}$, 2H), 1.45- 1.39 (m, 2H), $1.35-1.22$ (comp, 4H), $0.88(\mathrm{t}, J=5.1 \mathrm{~Hz}, 3 \mathrm{H}) ;{ }^{13} \mathrm{C} \mathrm{NMR}$ $\left(100 \mathrm{MHz}, \mathrm{CDCl}_{3}\right)(\delta, \mathrm{ppm}) 146.2,139.8,132.4,128.38,128.35,127.6,122.1,111.8$, 89.0, 80.5, 73.1, 70.1, 68.5, 33.2, 31.7, 27.3, 22.6, 20.5, 14.1; HRMS (TOF MS ESI ${ }^{+}$) calculated for $\mathrm{C}_{19} \mathrm{H}_{27} \mathrm{NO}_{4} \mathrm{SNa}[\mathrm{M}+\mathrm{Na}]^{+}: 388.1553$, found 388.1549 .

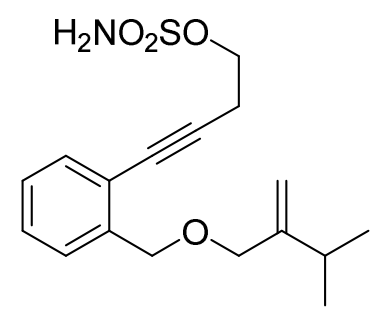

4-\{2-[(3-Methyl-2-methylenebutoxy)methyl]phenyl\}but-3-yn-1-yl sulfamate (1q). Yellow oil. ${ }^{1} \mathrm{H}$ NMR $\left(400 \mathrm{MHz}, \mathrm{CDCl}_{3}\right)(\delta, \mathrm{ppm}) 7.42-7.40(\mathrm{~m}, 2 \mathrm{H}), 7.32$ - $7.28(\mathrm{~m}$, 1H), $7.24-7.20(\mathrm{~m}, 1 \mathrm{H}), 5.46(\mathrm{~s}, 2 \mathrm{H}), 5.06(\mathrm{~d}, J=1.0 \mathrm{~Hz}, 1 \mathrm{H}), 4.96(\mathrm{~s}, 1 \mathrm{H}), 4.63(\mathrm{~s}$, 2H), $4.31(\mathrm{t}, J=6.6 \mathrm{~Hz}, 2 \mathrm{H}), 4.05(\mathrm{~s}, 2 \mathrm{H}), 2.87(\mathrm{t}, J=6.6 \mathrm{~Hz}, 2 \mathrm{H}), 2.35(\mathrm{~m}, 1 \mathrm{H}), 1.06$ $(\mathrm{d}, J=6.9 \mathrm{~Hz}, 6 \mathrm{H}) ;{ }^{13} \mathrm{C} \mathrm{NMR}\left(100 \mathrm{MHz}, \mathrm{CDCl}_{3}\right)(\delta, \mathrm{ppm}) 151.9,139.8,132.4$, $128.34,128.28,127.5,122.0,109.8,89.0,80.5,72.3,70.1,68.4,30.9,21.7,20.5$; HRMS (TOF MS ESI ${ }^{+}$) calculated for $\mathrm{C}_{17} \mathrm{H}_{23} \mathrm{NO}_{4} \mathrm{SNa}[\mathrm{M}+\mathrm{Na}]^{+}$: 360.1240 , found 360.1229 . 


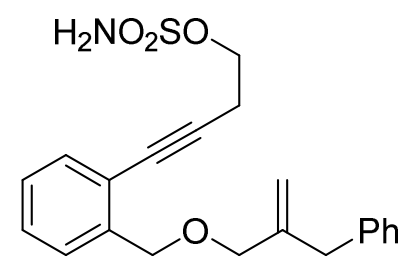

4-\{2-[((2-Benzylallyl)oxy)methyl]phenyl\}but-3-yn-1-yl sulfamate (1r). Yellow oil. ${ }^{1} \mathrm{H}$ NMR $\left(400 \mathrm{MHz}, \mathrm{CDCl}_{3}\right)(\delta, \mathrm{ppm}) 7.44-7.42(\mathrm{~m}, 1 \mathrm{H}), 7.37-7.35(\mathrm{~m}, 1 \mathrm{H}), 7.31$ - 7.25 (comp, 3H), $7.24-7.18$ (comp, 4H), 5.32 (s, 2H), 5.17 (s, 1H), 4.97 (s, 1H), $4.62(\mathrm{~s}, 2 \mathrm{H}), 4.31(\mathrm{t}, J=6.6 \mathrm{~Hz}, 2 \mathrm{H}), 3.95(\mathrm{~s}, 2 \mathrm{H}), 3.42(\mathrm{~s}, 2 \mathrm{H}), 2.87(\mathrm{t}, J=6.5 \mathrm{~Hz}$, $2 \mathrm{H}) ;{ }^{13} \mathrm{C} \mathrm{NMR}\left(100 \mathrm{MHz}, \mathrm{CDCl}_{3}\right)(\delta, \mathrm{ppm}) 145.4,139.7,139.1,132.4,129.1,128.5$, $128.44,128.35,127.6,126.3,122.2,114.1,89.0,80.5,72.3,70.2,68.4,39.9,20.5$; HRMS (TOF MS ESI ${ }^{+}$) calculated for $\mathrm{C}_{21} \mathrm{H}_{23} \mathrm{NO}_{4} \mathrm{SNa}[\mathrm{M}+\mathrm{Na}]^{+}: 408.1240$, found 408.1247 .

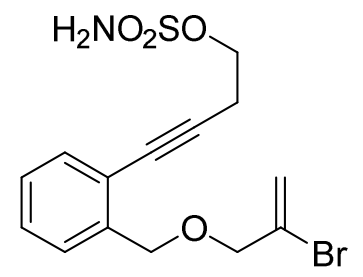

4-\{2-[((2- Bromoallyl)oxy)methyl]phenyl\}but-3-yn-1-yl sulfamate (1s). Yellow oil. ${ }^{1} \mathrm{H}$ NMR $\left(400 \mathrm{MHz}, \mathrm{CDCl}_{3}\right)(\delta, \mathrm{ppm}) 7.43-7.35(\mathrm{~m}, 2 \mathrm{H}), 7.30-7.26(\mathrm{~m}, 1 \mathrm{H}), 7.23$ $-7.19(\mathrm{~m}, 1 \mathrm{H}), 5.94(\mathrm{~d}, J=1.4 \mathrm{~Hz}, 1 \mathrm{H}), 5.63(\mathrm{~d}, J=0.8 \mathrm{~Hz}, 1 \mathrm{H}), 5.34(\mathrm{~s}, 2 \mathrm{H}), 4.66(\mathrm{~s}$ $2 \mathrm{H}), 4.30(\mathrm{t}, J=6.6 \mathrm{~Hz}, 2 \mathrm{H}), 4.14(\mathrm{~s}, 2 \mathrm{H}), 2.85(\mathrm{t}, J=6.6 \mathrm{~Hz}, 2 \mathrm{H}) ;{ }^{13} \mathrm{C}$ NMR $(100$ $\left.\mathrm{MHz}, \mathrm{CDCl}_{3}\right)(\delta, \mathrm{ppm}) 139.0,132.5,129.3,128.53,128.50,127.9,122.2,118.6,89.2$, 80.4, 74.2, 70.3, 68.6, 20.6; HRMS (TOF MS ESI ${ }^{+}$) calculated for $\mathrm{C}_{14} \mathrm{H}_{16} \mathrm{BrNO}_{4} \mathrm{SNa}$ $[\mathrm{M}+\mathrm{Na}]^{+}:$395.9876, found 395.9859 .<smiles>C=C(I)COCc1ccccc1C#CCCOS(N)(=O)=O</smiles>

4-\{2-[((2-Iodoallyl)oxy)methyl]phenyl\}but-3-yn-1-yl sulfamate (1t). Yellow oil. ${ }^{1} \mathrm{H}$ NMR (400 MHz, $\left.\mathrm{CDCl}_{3}\right)(\delta, \mathrm{ppm}) 7.43(\mathrm{t}, J=6.9 \mathrm{~Hz}, 2 \mathrm{H}), 7.35-7.30(\mathrm{~m}, 1 \mathrm{H}), 7.27$ 
$-7.24(\mathrm{~m}, 1 \mathrm{H}), 6.45(\mathrm{~d}, J=1.5 \mathrm{~Hz}, 1 \mathrm{H}), 5.96(\mathrm{~s}, 1 \mathrm{H}), 5.15(\mathrm{~s}, 2 \mathrm{H}), 4.68(\mathrm{~s}, 2 \mathrm{H}), 4.37$ (t, $J=6.5 \mathrm{~Hz}, 2 \mathrm{H}), 4.13(\mathrm{~s}, 2 \mathrm{H}), 2.91(\mathrm{t}, J=6.5 \mathrm{~Hz}, 2 \mathrm{H}) ;{ }^{13} \mathrm{C} \mathrm{NMR}\left(100 \mathrm{MHz}, \mathrm{CDCl}_{3}\right)$ ( $\delta$, ppm) 139.0, 132.5, 128.8, 128.6, 128.0, 126.9, 122.3, 107.1, 89.2, 80.5, 70.2, 68.7, 68.6, 20.7; HRMS (TOF MS ESI ${ }^{+}$) calculated for $\mathrm{C}_{14} \mathrm{H}_{16} \mathrm{INO}_{4} \mathrm{SNa}[\mathrm{M}+\mathrm{Na}]^{+}$: 443.9737, found 443.9728 .

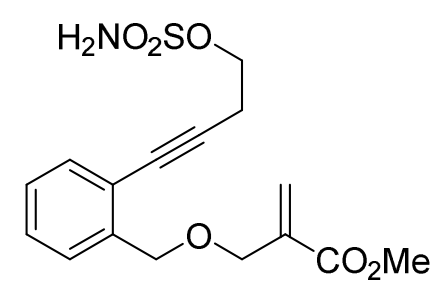

Methyl 2-\{[(2-(4-(sulfamoyloxy)but-1-yn-1-yl)benzyl)oxy]methyl\}acrylate (1u). Colorless oil. ${ }^{1} \mathrm{H}$ NMR $\left(400 \mathrm{MHz}, \mathrm{CDCl}_{3}\right)(\delta, \mathrm{ppm}) 7.42-7.39(\mathrm{~m}, 2 \mathrm{H}), 7.31$ (t, $J=$ $7.3 \mathrm{~Hz}, 1 \mathrm{H}), 7.23$ (t, $J=7.5 \mathrm{~Hz}, 1 \mathrm{H}), 6.32(\mathrm{~s}, 1 \mathrm{H}), 5.93$ (d, $J=0.9 \mathrm{~Hz}, 1 \mathrm{H}), 5.42$ (s, 2H), $4.70(\mathrm{~s}, 2 \mathrm{H}), 4.33(\mathrm{t}, J=6.6 \mathrm{~Hz}, 2 \mathrm{H}), 4.27(\mathrm{~s}, 2 \mathrm{H}), 3.76(\mathrm{~s}, 3 \mathrm{H}), 2.88(\mathrm{t}, J=6.6$ $\mathrm{Hz}, 2 \mathrm{H}) ;{ }^{13} \mathrm{C} \mathrm{NMR}\left(100 \mathrm{MHz}, \mathrm{CDCl}_{3}\right)(\delta, \mathrm{ppm}) 166.9,139.6,137.0,132.3,128.5$, 128.4, 127.8, 127.0, 122.3, 89.2, 80.4, 70.9, 68.6, 68.4, 52.2, 20.6; HRMS (TOF MS $\mathrm{ESI}^{+}$) calculated for $\mathrm{C}_{16} \mathrm{H}_{19} \mathrm{NO}_{6} \mathrm{SNa}[\mathrm{M}+\mathrm{Na}]^{+}: 376.0825$, found 376.0835 .

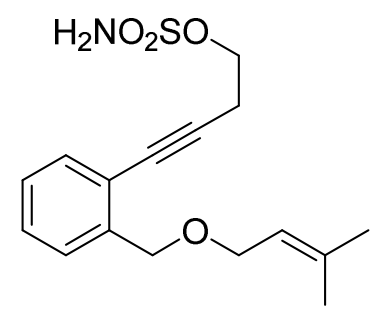

\section{4-\{2-[((3-Methylbut-2-en-1-yl)oxy)methyl]phenyl\}but-3-yn-1-yl sulfamate (1v).}

Yellow oil. ${ }^{1} \mathrm{H}$ NMR $\left(400 \mathrm{MHz}, \mathrm{CDCl}_{3}\right)(\delta$, ppm) 7.42 - $7.38(\mathrm{~m}, 2 \mathrm{H}), 7.31-7.27(\mathrm{~m}$, 1H), $7.24-7.20(\mathrm{~m}, 1 \mathrm{H}), 5.53(\mathrm{~s}, 2 \mathrm{H}), 5.41-5.37(\mathrm{~m}, 1 \mathrm{H}), 4.63(\mathrm{~s}, 2 \mathrm{H}), 4.30(\mathrm{t}, J=$ $6.5 \mathrm{~Hz}, 2 \mathrm{H}), 4.02$ (d, $J=7.0 \mathrm{~Hz}, 2 \mathrm{H}), 2.86(\mathrm{t}, J=6.5 \mathrm{~Hz}, 2 \mathrm{H}), 1.75$ (s, 3H), 1.65 (s, $3 \mathrm{H}) ;{ }^{13} \mathrm{C}$ NMR $\left(100 \mathrm{MHz}, \mathrm{CDCl}_{3}\right)(\delta, \mathrm{ppm}) 139.8,137.9,132.4,128.6,128.3,127.6$, 122.3, 120.7, 89.0, 80.5, 70.2, 68.4, 66.6, 25.8, 20.5, 18.1; HRMS (TOF MS ESI ${ }^{+}$) calculated for $\mathrm{C}_{16} \mathrm{H}_{21} \mathrm{NO}_{4} \mathrm{SNa}[\mathrm{M}+\mathrm{Na}]^{+}$: 346.1083, found 346.1093 . 


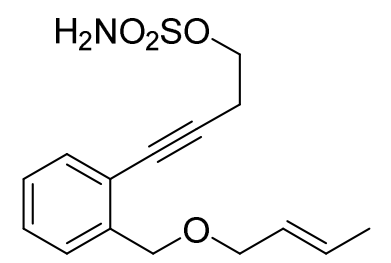

(E)-4-\{2-[(But-2-en-1-yloxy)methyl]phenyl\}but-3-yn-1-yl sulfamate (1w). Yellow oil. ${ }^{1} \mathrm{H}$ NMR (400 MHz, $\left.\mathrm{CDCl}_{3}\right)(\delta, \mathrm{ppm}) 7.41-7.37$ (m, 2H), $7.31-7.27(\mathrm{~m}, 1 \mathrm{H})$, $7.24-7.20(\mathrm{~m}, 1 \mathrm{H}), 5.79-5.70(\mathrm{~m}, 1 \mathrm{H}), 5.65-5.57(\mathrm{~m}, 1 \mathrm{H}), 5.43(\mathrm{~s}, 2 \mathrm{H}), 4.62(\mathrm{~s}$, 2H), $4.32(\mathrm{t}, J=6.5 \mathrm{~Hz}, 2 \mathrm{H}), 3.98-3.96(\mathrm{~m}, 2 \mathrm{H}), 2.87(\mathrm{t}, J=6.5 \mathrm{~Hz}, 2 \mathrm{H}), 1.71(\mathrm{dd}, J$ $=6.3,1.2 \mathrm{~Hz}, 3 \mathrm{H}) ;{ }^{13} \mathrm{C} \mathrm{NMR}\left(100 \mathrm{MHz}, \mathrm{CDCl}_{3}\right)(\delta, \mathrm{ppm}) 139.8,132.4,130.4,128.7$, 128.3, 127.7, 127.3, 122.3, 89.0, 80.5, 71.0, 70.1, 68.4, 20.6, 17.9; HRMS (TOF MS ESI $^{+}$) calculated for $\mathrm{C}_{15} \mathrm{H}_{20} \mathrm{NO}_{4} \mathrm{~S}[\mathrm{M}+\mathrm{H}]^{+}: 310.1108$, found 310.1109 .

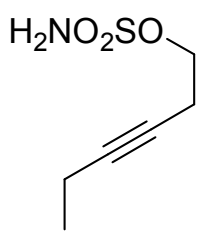

Hex-3-yn-1-yl sulfamate (1x). ${ }^{\mathbf{1 b}}{ }^{1} \mathrm{H}$ NMR $\left(400 \mathrm{MHz}, \mathrm{CDCl}_{3}\right)(\delta$, ppm) 5.07 (s, 2H), $4.24(\mathrm{t}, J=7.0 \mathrm{~Hz}, 2 \mathrm{H}), 2.64-2.59$ (m, 2H), $2.19-2.12(\mathrm{~m}, 2 \mathrm{H}), 1.10$ (t, $J=7.5 \mathrm{~Hz}$, $3 \mathrm{H}) ;{ }^{13} \mathrm{C}$ NMR (100 MHz, $\left.\mathrm{CDCl}_{3}\right)(\delta, \mathrm{ppm}) 84.5,73.8,69.3,19.8,14.1,12.4$. 


\section{General Procedure for the Asymmetric Cascade Reaction}

To a $10-\mathrm{mL}$ oven-dried vial containing a magnetic stirring bar, $\mathrm{Rh}_{2}(S \text {-TCPTTL })_{4}(3.6$ $\mathrm{mg}, 1.0 \mathrm{~mol} \%), \mathrm{PhI}(\mathrm{OAc})_{2}(70.8 \mathrm{mg}, 1.1$ equiv) and $4 \AA \mathrm{MS}(100 \mathrm{mg})$ in mixed solvent $\left(\mathrm{C}_{6} \mathrm{H}_{5} \mathrm{CF}_{3}: \mathrm{TBME}=10: 1,0.5 \mathrm{~mL}\right)$, was added as a solution of sulfamate ester $1(0.2 \mathrm{mmol})$ in the same mixed solvent $(1.0 \mathrm{~mL})$ via a syringe pump over $1 \mathrm{~h}$ under argon atmosphere at $0{ }^{\circ} \mathrm{C}$. After addition, the reaction mixture was stirred for additional $60 \mathrm{~h}$ (or with indicated times) under these conditions until consumption of the material (monitored by TLC). Then the reaction mixture was purified by column chromatography on silica gel without any additional treatment $($ Hexanes : EtOAc $=$ $4: 1$ to $2: 1$ ) to give the pure products 2 in good to high yields and excellent enantioselectivity.<smiles>C=CC[C@]12CCOS(=O)(=O)N=C1c1ccccc1CO2</smiles>

\section{(S)-5a-Allyl-5,5a-dihydro-4H,7H-isochromeno[4,3-d][1,2,3]oxathiazepine}

2,2-dioxide (2a). ${ }^{1}$ Colorless oil. $45.1 \mathrm{mg}, 77 \%$ yield. $92 \%$ ee, $[\alpha]_{\mathrm{D}}{ }^{20}=296^{\circ}(\mathrm{c}=0.1$, DCM); ${ }^{1} \mathrm{H}$ NMR $\left(400 \mathrm{MHz}, \mathrm{CDCl}_{3}\right)(\delta, \mathrm{ppm}) 8.29$ - $8.27(\mathrm{~m}, 1 \mathrm{H}), 7.59$ - $7.55(\mathrm{~m}$, 1H), 7.38 (t, $J=7.7 \mathrm{~Hz}, 1 \mathrm{H}), 7.17(\mathrm{~d}, J=7.7 \mathrm{~Hz}, 1 \mathrm{H}), 5.94-5.84(\mathrm{~m}, 1 \mathrm{H}), 5.36-$ $5.31(\mathrm{~m}, 2 \mathrm{H}), 5.09$ (d, $J=16.5 \mathrm{~Hz}, 1 \mathrm{H}), 4.86(\mathrm{~d}, J=16.5 \mathrm{~Hz}, 1 \mathrm{H}), 4.49-4.37(\mathrm{~m}$, 2H), $2.87-2.68$ (comp, 3H), 2.19 (ddd, $J=15.6,4.2,1.6 \mathrm{~Hz}, 1 \mathrm{H}) ;{ }^{13} \mathrm{C}$ NMR $(100$ $\left.\mathrm{MHz}, \mathrm{CDCl}_{3}\right)(\delta, \mathrm{ppm}) 173.3,139.8,134.6,130.3,129.2,128.2,127.7,124.2,120.8$, 80.2, 65.2, 61.9, 34.2, 32.6; HPLC conditions for determination of enantiomeric excess: Daicel Chiralcel IA, $\lambda=254 \mathrm{~nm}$, hexane : 2-propanol $=90: 10$, flow rate $=1.0$ $\mathrm{mL} / \mathrm{min}, t_{\text {minor }}=12.4 \mathrm{~min}, t_{\text {major }}=14.1 \mathrm{~min}$. 
<smiles>C=CC[C@]12CCOS(=O)(=O)N=C1c1ccc(F)cc1CO2</smiles>

(S)-5a-Allyl-9-fluoro-5,5a-dihydro-4H,7H-isochromeno[4,3-d][1,2,3]oxathiazepin e 2,2-dioxide (2b). Colorless oil. $42.3 \mathrm{mg}, 68 \%$ yield. $94 \% e e,[\alpha]_{\mathrm{D}}{ }^{20}=316^{\circ}(\mathrm{c}=0.1$, DCM); ${ }^{1} \mathrm{H}$ NMR (400 MHz, $\left.\mathrm{CDCl}_{3}\right)(\delta, \mathrm{ppm}) 8.32(\mathrm{dd}, J=9.0,5.6 \mathrm{~Hz}, 1 \mathrm{H}), 7.09-$ $7.04(\mathrm{~m}, 1 \mathrm{H}), 6.88-6.85(\mathrm{~m}, 1 \mathrm{H}), 5.92-5.81(\mathrm{~m}, 1 \mathrm{H}), 5.36-5.31(\mathrm{~m}, 2 \mathrm{H}), 5.06(\mathrm{~d}$, $J=16.7 \mathrm{~Hz}, 1 \mathrm{H}), 4.84(\mathrm{~d}, J=16.7 \mathrm{~Hz}, 1 \mathrm{H}), 4.49-4.36(\mathrm{~m}, 2 \mathrm{H}), 2.85-2.69(\mathrm{~m}, 3 \mathrm{H})$, $2.21-2.16(\mathrm{~m}, 1 \mathrm{H}) ;{ }^{13} \mathrm{C}$ NMR $\left(100 \mathrm{MHz}, \mathrm{CDCl}_{3}\right)(\delta, \mathrm{ppm}) 172.1,166.4(\mathrm{~d}, J=259.2$ Hz), 142.8 (d, $J=8.9 \mathrm{~Hz}), 132.6$ (d, $J=9.9 \mathrm{~Hz}), 130.1,124.1$ (d, $J=2.7 \mathrm{~Hz}), 121.0$, $116.2(\mathrm{~d}, J=22.3 \mathrm{~Hz}), 110.9(\mathrm{~d}, J=22.6 \mathrm{~Hz}), 80.2,65.2,61.7(\mathrm{~d}, J=2.0 \mathrm{~Hz}), 34.1$, 32.5; ${ }^{19} \mathrm{~F}$ NMR (376 MHz, $\left.\mathrm{CDCl}_{3}\right)(\delta, \mathrm{ppm})$-101.57; HRMS (TOF MS $\mathrm{ESI}^{+}$) calculated for $\mathrm{C}_{14} \mathrm{H}_{15} \mathrm{FNO}_{4} \mathrm{~S}[\mathrm{M}+\mathrm{H}]^{+}:$312.0700, found 312.0697; HPLC conditions for determination of enantiomeric excess: Daicel Chiralcel IA, $\lambda=254 \mathrm{~nm}$, hexane : 2-propanol $=90: 10$, flow rate $=1.0 \mathrm{~mL} / \mathrm{min}, t_{\text {minor }}=12.8 \mathrm{~min}, t_{\text {major }}=15.6 \mathrm{~min}$.<smiles>C=CC[C@@]12CCOS(=O)(=O)N=C1c1ccc(Cl)cc1CO2</smiles>

(S)-5a-Allyl-9-chloro-5,5a-dihydro-4H,7H-isochromeno $[4,3-d][1,2,3]$ oxathiazepin e 2,2-dioxide (2c). Colorless oil. $41.3 \mathrm{mg}, 63 \%$ yield. $94 \%$ ee, $[\alpha]_{\mathrm{D}}{ }^{20}=346^{\circ}(\mathrm{c}=0.1$, DCM); ${ }^{1} \mathrm{H}$ NMR $\left(400 \mathrm{MHz}, \mathrm{CDCl}_{3}\right)(\delta, \mathrm{ppm}) 8.24(\mathrm{~d}, J=8.7 \mathrm{~Hz}, 1 \mathrm{H}), 7.35(\mathrm{~d}, J=$ $8.6 \mathrm{~Hz}, 1 \mathrm{H}), 7.18(\mathrm{~s}, 1 \mathrm{H}), 5.92-5.82(\mathrm{~m}, 1 \mathrm{H}), 5.36-5.32(\mathrm{~m}, 2 \mathrm{H}), 5.05(\mathrm{~d}, J=16.6$ $\mathrm{Hz}, 1 \mathrm{H}), 4.83$ (d, J = 16.7 Hz, 1H), $4.50-4.37$ (m, 2H), $2.84-2.69$ (m, 3H), $2.21-$ $2.17(\mathrm{~m}, 1 \mathrm{H}) ;{ }^{13} \mathrm{C} \mathrm{NMR}\left(100 \mathrm{MHz}, \mathrm{CDCl}_{3}\right)(\delta, \mathrm{ppm})$ 172.2, 141.3, 141.2, 130.9, 130.0, 128.8, 126.3, 124.3, 121.1, 80.2, 65.2, 61.5, 34.2, 32.5; HRMS (TOF MS ESI ${ }^{+}$) calculated for $\mathrm{C}_{14} \mathrm{H}_{14} \mathrm{ClNO}_{4} \mathrm{SNa}[\mathrm{M}+\mathrm{Na}]^{+}$: 350.0224, found 350.0209; HPLC 
conditions for determination of enantiomeric excess: Daicel Chiralcel IA, $\lambda=254 \mathrm{~nm}$, hexane $: 2$-propanol $=90: 10$, flow rate $=1.0 \mathrm{~mL} / \mathrm{min}, t_{\text {minor }}=13.3 \mathrm{~min}, t_{\text {major }}=16.7$ $\min$.

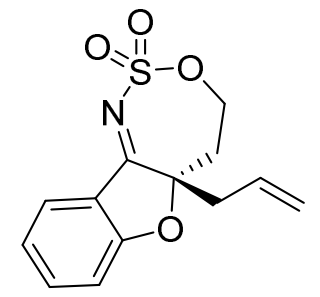

(S)-5a-Allyl-5,5a-dihydro-4H-benzofuro[3,2-d][1,2,3]oxathiazepine

2,2-dioxide

(2d). White solid, $\mathrm{mp}=95-96{ }^{\circ} \mathrm{C} .45 .2 \mathrm{mg}, 81 \%$ yield. $90 \%$ ee [after recrystallization in a mixture of DCM, ethyl acetate and hexanes: 54\% yield, $>99.5 \%$ $e e,[\alpha]_{\mathrm{D}}{ }^{20}=384^{\circ}(\mathrm{c}=0.1, \mathrm{DCM})$. White solid, $\left.\mathrm{mp}=95-96{ }^{\circ} \mathrm{C}\right] ;{ }^{1} \mathrm{H} \mathrm{NMR}(400 \mathrm{MHz}$, $\left.\mathrm{CDCl}_{3}\right)(\delta, \mathrm{ppm}) 7.74(\mathrm{~d}, J=7.9 \mathrm{~Hz}, 1 \mathrm{H}), 7.68-7.63(\mathrm{~m}, 1 \mathrm{H}), 7.12(\mathrm{t}, J=7.5 \mathrm{~Hz}$, 1H), $7.03(\mathrm{~d}, J=8.4 \mathrm{~Hz}, 1 \mathrm{H}), 5.74-5.64(\mathrm{~m}, 1 \mathrm{H}), 5.29(\mathrm{~d}, J=17.1 \mathrm{~Hz}, 1 \mathrm{H}), 5.17(\mathrm{~d}$, $J=10.1 \mathrm{~Hz}, 1 \mathrm{H}), 4.93-4.86(\mathrm{~m}, 1 \mathrm{H}), 4.55-4.50(\mathrm{~m}, 1 \mathrm{H}), 3.17-3.11(\mathrm{~m}, 1 \mathrm{H}), 3.04$ - $2.99(\mathrm{~m}, 1 \mathrm{H}), 2.44-2.30(\mathrm{~m}, 2 \mathrm{H}) ;{ }^{13} \mathrm{C} \mathrm{NMR}\left(100 \mathrm{MHz}, \mathrm{CDCl}_{3}\right)(\delta, \mathrm{ppm})$ 183.8, 169.0, 139.6, 129.9, 125.6, 123.2, 121.6, 121.5, 112.7, 93.7, 68.0, 39.3, 32.0; HRMS (TOF MS ESI ${ }^{+}$) calculated for $\mathrm{C}_{13} \mathrm{H}_{13} \mathrm{NO}_{4} \mathrm{SNa}[\mathrm{M}+\mathrm{Na}]^{+}$: 302.0457, found 302.0460; HPLC conditions for determination of enantiomeric excess: Daicel Chiralpak AD-H, $\lambda=254 \mathrm{~nm}$, hexane $: 2$-propanol $=95: 5$, flow rate $=1.0 \mathrm{~mL} / \mathrm{min}, t_{\text {minor }}=21.4 \mathrm{~min}$, $t_{\text {major }}=23.5 \mathrm{~min}$.<smiles>C=C(C[C@]12COS(=O)(=O)N=C1c1ccccc1CO2)c1ccccc1</smiles>

(S)-5a-(2-Phenylallyl)-5,5a-dihydro-4H,7H-isochromeno[4,3-d][1,2,3] oxathiazepi ne 2,2-dioxide (2e). Colorless oil. $38.4 \mathrm{mg}, 52 \%$ yield. $91 \% e e,[\alpha]_{\mathrm{D}}{ }^{20}=148^{\circ}(\mathrm{c}=0.1$, DCM); ${ }^{1} \mathrm{H}$ NMR $\left(400 \mathrm{MHz}, \mathrm{CDCl}_{3}\right)(\delta, \mathrm{ppm}) 8.27-8.25(\mathrm{~m}, 1 \mathrm{H}), 7.58-7.54(\mathrm{~m}$, 
1H), $7.38(\mathrm{t}, J=7.7 \mathrm{~Hz}, 1 \mathrm{H}), 7.35-7.27$ (comp, 5H), 7.15 (d, $J=7.5 \mathrm{~Hz}, 1 \mathrm{H}), 5.61$ (s, 1H), $5.49(\mathrm{~s}, 1 \mathrm{H}), 5.08(\mathrm{~d}, J=16.5 \mathrm{~Hz}, 1 \mathrm{H}), 4.78(\mathrm{~d}, J=16.5 \mathrm{~Hz}, 1 \mathrm{H}), 4.36-4.31(\mathrm{~m}$, 1H), $4.24-4.17$ (m, 1H), 3.21 (d, $J=16.9 \mathrm{~Hz}, 1 \mathrm{H}), 3.09$ (d, $J=16.9 \mathrm{~Hz}, 1 \mathrm{H}), 2.83-$ $2.74(\mathrm{~m}, 1 \mathrm{H}), 2.44-2.38(\mathrm{~m}, 1 \mathrm{H}) ;{ }^{13} \mathrm{C} \mathrm{NMR}\left(100 \mathrm{MHz}, \mathrm{CDCl}_{3}\right)(\delta, \mathrm{ppm})$ 173.4, $143.0,142.3,140.1,134.5,129.3,128.7,128.2,128.1,127.9,126.3,124.1,117.7$, 81.9, 65.5, 62.1, 34.1, 32.7; HRMS (TOF MS ESI ${ }^{+}$) calculated for $\mathrm{C}_{20} \mathrm{H}_{19} \mathrm{NO}_{4} \mathrm{SNa}[\mathrm{M}$ $+\mathrm{Na}^{+}$: 392.0927, found 392.0945; HPLC conditions for determination of enantiomeric excess: Daicel Chiralcel IA, $\lambda=254 \mathrm{~nm}$, hexane : 2-propanol $=90: 10$, flow rate $=1.0 \mathrm{~mL} / \mathrm{min}, t_{\text {minor }}=17.8 \mathrm{~min}, t_{\text {major }}=22.9 \mathrm{~min}$.<smiles>C=C(C[C@@]12COS(=O)(=O)N=C1c1ccccc1CO2)c1ccc(C(F)(F)F)cc1</smiles>

(S)-5a-\{2-[4-(Trifluoromethyl)phenyl]allyl\}-5,5a-dihydro-4H,7H-isochromeno[4, 3-d][1,2,3]oxathiazepine 2,2-dioxide (2f). Colorless oil. $49.8 \mathrm{mg}, 57 \%$ yield. 90\% ee, $[\alpha]_{\mathrm{D}}{ }^{20}=175^{\circ}(\mathrm{c}=0.1, \mathrm{DCM}) ;{ }^{1} \mathrm{H}$ NMR $\left(400 \mathrm{MHz}, \mathrm{CDCl}_{3}\right)(\delta, \mathrm{ppm}) 8.20-8.18(\mathrm{~m}$, 1H), $7.58-7.55$ (comp, 3H), 7.39 - 7.35 (comp, 3H), 7.14 (d, $J=7.6 \mathrm{~Hz}, 1 \mathrm{H}), 5.70$ (s, $1 \mathrm{H}), 5.53(\mathrm{~s}, 1 \mathrm{H}), 4.99(\mathrm{~d}, J=16.4 \mathrm{~Hz}, 1 \mathrm{H}), 4.77(\mathrm{~d}, J=16.4 \mathrm{~Hz}, 1 \mathrm{H}), 4.41-4.36(\mathrm{~m}$, 1H), $4.29-4.22$ (m, 1H), 3.30 (d, $J=16.7 \mathrm{~Hz}, 1 \mathrm{H}), 3.09$ (d, $J=16.7 \mathrm{~Hz}, 1 \mathrm{H}), 2.84-$ $2.76(\mathrm{~m}, 1 \mathrm{H}), 2.42-2.36(\mathrm{~m}, 1 \mathrm{H}) ;{ }^{13} \mathrm{C} \mathrm{NMR}\left(100 \mathrm{MHz}, \mathrm{CDCl}_{3}\right)(\delta, \mathrm{ppm}) 173.4$, 145.7, 141.9, 140.0, 134.6, 130.0 (q, $J=32.8 \mathrm{~Hz}$ ), 129.1, 128.3, 128.1, 126.7, 125.6 (q, $J=3.7 \mathrm{~Hz}), 124.12,124.10$ (q, $J=272.2 \mathrm{~Hz}), 119.7,81.9,65.4,62.1,34.2,33.5$; ${ }^{19} \mathrm{~F}$ NMR $\left(376 \mathrm{MHz}, \mathrm{CDCl}_{3}\right)(\delta, \mathrm{ppm})-62.54$; HRMS (TOF MS ESI ${ }^{+}$) calculated for $\mathrm{C}_{21} \mathrm{H}_{18} \mathrm{~F}_{3} \mathrm{NO}_{4} \mathrm{SNa}[\mathrm{M}+\mathrm{Na}]^{+}:$460.0801, found 460.0816; HPLC conditions for determination of enantiomeric excess: Daicel Chiralcel IA, $\lambda=254 \mathrm{~nm}$, hexane : 2-propanol $=90: 10$, flow rate $=1.0 \mathrm{~mL} / \mathrm{min}, t_{\text {minor }}=15.7 \mathrm{~min}, t_{\text {major }}=21.2 \mathrm{~min}$. 
<smiles>C=C(C[C@]12COS(=O)(=O)N=C1c1ccccc1CO2)c1ccc(Cl)cc1</smiles>

(S)-5a-[2-(4-Chlorophenyl)allyl]-5,5a-dihydro-4H,7H-isochromeno[4,3-d] [1,2,3]0 xathiazepine 2,2-dioxide (2g). Colorless oil. $46.8 \mathrm{mg}, 58 \%$ yield. $94 \%$ ee, $[\alpha]_{\mathrm{D}}{ }^{20}=$ $133^{\circ}(\mathrm{c}=0.05, \mathrm{DCM}) ;{ }^{1} \mathrm{H}$ NMR $\left(400 \mathrm{MHz}, \mathrm{CDCl}_{3}\right)(\delta, \mathrm{ppm}) 8.22(\mathrm{~d}, J=7.6 \mathrm{~Hz}, 1 \mathrm{H})$, $7.58-7.54(\mathrm{~m}, 1 \mathrm{H}), 7.38(\mathrm{t}, J=7.4 \mathrm{~Hz}, 1 \mathrm{H}), 7.29-7.26(\mathrm{~m}, 2 \mathrm{H}), 7.24-7.21(\mathrm{~m}, 2 \mathrm{H})$, $7.15(\mathrm{~d}, J=7.7 \mathrm{~Hz}, 1 \mathrm{H}), 5.61(\mathrm{~s}, 1 \mathrm{H}), 5.46(\mathrm{~s}, 1 \mathrm{H}), 5.02(\mathrm{~d}, J=16.4 \mathrm{~Hz}, 1 \mathrm{H}), 4.78(\mathrm{~d}$, $J=16.4 \mathrm{~Hz}, 1 \mathrm{H}), 4.39-4.34(\mathrm{~m}, 1 \mathrm{H}), 4.26-4.19(\mathrm{~m}, 1 \mathrm{H}), 3.21(\mathrm{~d}, J=16.7 \mathrm{~Hz}, 1 \mathrm{H})$, $3.05(\mathrm{~d}, J=16.7 \mathrm{~Hz}, 1 \mathrm{H}), 2.83-2.74(\mathrm{~m}, 1 \mathrm{H}), 2.41-2.36(\mathrm{~m}, 1 \mathrm{H}) ;{ }^{13} \mathrm{C}$ NMR $(100$ $\left.\mathrm{MHz}, \mathrm{CDCl}_{3}\right)(\delta, \mathrm{ppm}) 173.3,141.9,140.6,140.0,134.6,134.0,129.2,128.8,128.2$, 128.0, 127.6, 124.1, 118.3, 81.9, 65.5, 62.1, 34.2, 33.1; HRMS (TOF MS ESI') calculated for $\mathrm{C}_{20} \mathrm{H}_{18} \mathrm{ClNO}_{4} \mathrm{SNa}[\mathrm{M}+\mathrm{Na}]^{+}:$426.0537, found 426.0545; HPLC conditions for determination of enantiomeric excess: Daicel Chiralcel IA, $\lambda=254 \mathrm{~nm}$, hexane $: 2$-propanol $=90: 10$, flow rate $=1.0 \mathrm{~mL} / \mathrm{min}, t_{\text {minor }}=18.3 \mathrm{~min}, t_{\text {major }}=26.2$ $\min$.<smiles>C=C(C[C@@H]1OCc2ccccc2/C1=N/S(=O)(=O)OC)c1ccc(Br)cc1</smiles>

(S)-5a-[2-(4-Bromophenyl)allyl]-5,5a-dihydro-4H,7H-isochromeno[4,3-d] $[1,2,3]$ o xathiazepine 2,2-dioxide (2h). Colorless oil. $48.4 \mathrm{mg}, 54 \%$ yield. $99 \%$ ee, $[\alpha]_{\mathrm{D}}{ }^{20}=$ $49^{\circ}(\mathrm{c}=0.05, \mathrm{DCM}) ;{ }^{1} \mathrm{H} \mathrm{NMR}\left(400 \mathrm{MHz}, \mathrm{CDCl}_{3}\right)(\delta, \mathrm{ppm}) 8.23(\mathrm{~d}, J=8.0 \mathrm{~Hz}, 1 \mathrm{H})$, $7.58-7.54$ (m, 1H), $7.45-7.36$ (comp, 3H), $7.18-7.13$ (comp, 3H), 5.61 (s, 1H), $5.47(\mathrm{~s}, 1 \mathrm{H}), 5.02(\mathrm{~d}, J=16.4 \mathrm{~Hz}, 1 \mathrm{H}), 4.78(\mathrm{~d}, J=16.4 \mathrm{~Hz}, 1 \mathrm{H}), 4.39-4.34(\mathrm{~m}, 1 \mathrm{H})$, $4.27-4.19(\mathrm{~m}, 1 \mathrm{H}), 3.22(\mathrm{~d}, J=16.7 \mathrm{~Hz}, 1 \mathrm{H}), 3.05(\mathrm{~d}, J=16.7 \mathrm{~Hz}, 1 \mathrm{H}), 2.83-2.74$ $(\mathrm{m}, 1 \mathrm{H}), 2.41-2.35(\mathrm{~m}, 1 \mathrm{H}) .{ }^{13} \mathrm{C} \mathrm{NMR}\left(100 \mathrm{MHz}, \mathrm{CDCl}_{3}\right)(\delta, \mathrm{ppm}) 173.4,141.9$, 
141.1, 140.0, 134.6, 131.8, 129.2, 128.2, 128.0, 127.9, 124.1, 122.1, 118.4, 81.9, 65.5, 62.1, 34.2, 33.0; HRMS (TOF MS ESI ${ }^{+}$) calculated for $\mathrm{C}_{20} \mathrm{H}_{18} \mathrm{BrNO}_{4} \mathrm{SNa}[\mathrm{M}+\mathrm{Na}]^{+}$: 470.0032, found 470.0036; HPLC conditions for determination of enantiomeric excess: Chiralpak IA-3, $\lambda=272 \mathrm{~nm}$, hexane : 2-propanol $=90: 10$, flow rate $=1.0$ $\mathrm{mL} / \mathrm{min}, t_{\text {minor }}=28.9 \mathrm{~min}, t_{\text {major }}=38.4 \mathrm{~min}$.<smiles>C=C(C[C@@]12CCOS(=O)(=O)N=C1c1ccccc1CO2)c1ccc(C)cc1</smiles>

(S)-5a-[2-(p-Tolyl)allyl]-5,5a-dihydro-4H,7H-isochromeno[4,3-d][1,2,3]oxathiaze pine 2,2-dioxide (2i). Colorless oil. $43.7 \mathrm{mg}, 57 \%$ yield. $96 \% e e,[\alpha]_{\mathrm{D}}{ }^{20}=138^{\circ}(\mathrm{c}=$ $0.1, \mathrm{DCM}) ;{ }^{1} \mathrm{H} \mathrm{NMR}\left(400 \mathrm{MHz}, \mathrm{CDCl}_{3}\right)(\delta, \mathrm{ppm}) 8.26(\mathrm{~d}, J=8.1 \mathrm{~Hz}, 1 \mathrm{H}), 7.58-$ $7.54(\mathrm{~m}, 1 \mathrm{H}), 7.37$ (t, $J=7.5 \mathrm{~Hz}, 1 \mathrm{H}), 7.21$ (d, $J=8.2 \mathrm{~Hz}, 2 \mathrm{H}), 7.17-7.12$ (comp, $3 \mathrm{H}), 5.56(\mathrm{~s}, 1 \mathrm{H}), 5.47(\mathrm{~s}, 1 \mathrm{H}), 5.10(\mathrm{~d}, J=16.5 \mathrm{~Hz}, 1 \mathrm{H}), 4.80(\mathrm{~d}, J=16.6 \mathrm{~Hz}, 1 \mathrm{H})$, $4.35-4.30(\mathrm{~m}, 1 \mathrm{H}), 4.23-4.16(\mathrm{~m}, 1 \mathrm{H}), 3.18(\mathrm{~d}, J=17.0 \mathrm{~Hz}, 1 \mathrm{H}), 3.06(\mathrm{~d}, J=16.9$ $\mathrm{Hz}, 1 \mathrm{H}), 2.82-2.73$ (m, 1H), $2.44-2.38$ (m, 1H), 2.34 (s, 3H); ${ }^{13} \mathrm{C}$ NMR (100 MHz, $\left.\mathrm{CDCl}_{3}\right)(\delta, \mathrm{ppm}) 173.4,142.7,140.1,139.4,137.9,134.5,129.4,129.3,128.1,127.9$, 126.1, 124.1, 116.9, 81.9, 65.5, 62.0, 34.1, 32.5, 21.2; HRMS (TOF MS ESI ${ }^{+}$) calculated for $\mathrm{C}_{21} \mathrm{H}_{21} \mathrm{NO}_{4} \mathrm{SNa}[\mathrm{M}+\mathrm{Na}]^{+}:$406.1083, found 406.1068; HPLC conditions for determination of enantiomeric excess: Daicel Chiralcel OD-H, $\lambda=254$ $\mathrm{nm}$, hexane $: 2$-propanol $=97: 3$, flow rate $=1.0 \mathrm{~mL} / \mathrm{min}, t_{\text {minor }}=38.3 \mathrm{~min}, t_{\text {major }}=$ $35.6 \mathrm{~min}$. 
<smiles>C=C(C[C@@]12CCOS(=O)(=O)N=C1c1ccccc1CO2)c1cccc(OC)c1</smiles>

(S)-5a-[2-(3-Methoxyphenyl)allyl]-5,5a-dihydro-4H,7H-isochromeno[4,3- $d][1,2,3]$ oxathiazepine 2,2-dioxide (2j). Colorless oil. $48.7 \mathrm{mg}, 61 \%$ yield. $92 \%$ ee, $[\alpha]_{\mathrm{D}}{ }^{20}=$ $126^{\circ}(\mathrm{c}=0.05, \mathrm{DCM}) ;{ }^{1} \mathrm{H} \mathrm{NMR}\left(400 \mathrm{MHz}, \mathrm{CDCl}_{3}\right)(\delta, \mathrm{ppm}) 8.25(\mathrm{~d}, J=8.0 \mathrm{~Hz}, 1 \mathrm{H})$, $7.58-7.54(\mathrm{~m}, 1 \mathrm{H}), 7.37(\mathrm{t}, J=7.6 \mathrm{~Hz}, 1 \mathrm{H}), 7.25-7.21(\mathrm{~m}, 1 \mathrm{H}), 7.16(\mathrm{~d}, J=7.7 \mathrm{~Hz}$, 1H), $6.90(\mathrm{~d}, J=7.9 \mathrm{~Hz}, 1 \mathrm{H}), 6.84-6.83(\mathrm{~m}, 2 \mathrm{H}), 5.58(\mathrm{~s}, 1 \mathrm{H}), 5.50(\mathrm{~s}, 1 \mathrm{H}), 5.09$ (d, $J=16.5 \mathrm{~Hz}, 1 \mathrm{H}), 4.80(\mathrm{~d}, J=16.5 \mathrm{~Hz}, 1 \mathrm{H}), 4.35-4.30(\mathrm{~m}, 1 \mathrm{H}), 4.25-4.18(\mathrm{~m}, 1 \mathrm{H})$, $3.80(\mathrm{~s}, 3 \mathrm{H}), 3.20(\mathrm{~d}, J=16.8 \mathrm{~Hz}, 1 \mathrm{H}), 3.08(\mathrm{~d}, J=16.8 \mathrm{~Hz}, 1 \mathrm{H}), 2.81-2.72(\mathrm{~m}, 1 \mathrm{H})$, $2.42-2.37(\mathrm{~m}, 1 \mathrm{H}) ;{ }^{13} \mathrm{C} \mathrm{NMR}\left(100 \mathrm{MHz}, \mathrm{CDCl}_{3}\right)(\delta, \mathrm{ppm}) 173.4,159.7,143.8$, $142.7,140.1,134.5,129.7,129.2,128.1,127.9,124.1,118.7,117.9,113.4,112.2$, 81.9, 65.5, 62.0, 55.4, 34.2, 32.8; HRMS (TOF MS ESI ${ }^{+}$) calculated for $\mathrm{C}_{21} \mathrm{H}_{21} \mathrm{NO}_{5} \mathrm{SNa}[\mathrm{M}+\mathrm{Na}]^{+}:$422.1033, found 422.1032; HPLC conditions for determination of enantiomeric excess: Daicel Chiralcel IA, $\lambda=254 \mathrm{~nm}$, hexane : 2-propanol $=90: 10$, flow rate $=1.0 \mathrm{~mL} / \mathrm{min}, t_{\text {minor }}=21.8 \mathrm{~min}, t_{\text {major }}=22.9 \mathrm{~min}$.<smiles>C=C(C[C@@]12COS(=O)(=O)N=C1c1ccccc1CO2)c1ccc(F)cc1</smiles>

(S)-5a-[2-(4-Fluorophenyl)allyl]-5,5a-dihydro-4H,7H-isochromeno[4,3-d][1,2,3]0 xathiazepine 2,2-dioxide (2k). Colorless oil. $40.2 \mathrm{mg}, 52 \%$ yield. $94 \% e e,[\alpha]_{\mathrm{D}}{ }^{20}=$ $256^{\circ}(\mathrm{c}=0.05, \mathrm{DCM}) ;{ }^{1} \mathrm{H} \mathrm{NMR}\left(400 \mathrm{MHz}, \mathrm{CDCl}_{3}\right)(\delta, \mathrm{ppm}) 8.23(\mathrm{~d}, J=7.6 \mathrm{~Hz}, 1 \mathrm{H})$, $7.58-7.54(\mathrm{~m}, 1 \mathrm{H}), 7.37(\mathrm{t}, J=7.6 \mathrm{~Hz}, 1 \mathrm{H}), 7.29-7.24(\mathrm{~m}, 2 \mathrm{H}), 7.15(\mathrm{~d}, J=7.6 \mathrm{~Hz}$, 1H), $7.02-6.96(\mathrm{~m}, 2 \mathrm{H}), 5.59(\mathrm{~s}, 1 \mathrm{H}), 5.44(\mathrm{~s}, 1 \mathrm{H}), 5.04$ (d, J=16.4 Hz, 1H), 4.78 (d, $J=16.5 \mathrm{~Hz}, 1 \mathrm{H}), 4.39-4.34(\mathrm{~m}, 1 \mathrm{H}), 4.26-4.19(\mathrm{~m}, 1 \mathrm{H}), 3.21(\mathrm{~d}, J=16.8 \mathrm{~Hz}, 1 \mathrm{H})$, 
$3.05(\mathrm{~d}, J=16.8 \mathrm{~Hz}, 1 \mathrm{H}), 2.83-2.74(\mathrm{~m}, 1 \mathrm{H}), 2.42-2.37(\mathrm{~m}, 1 \mathrm{H}) ;{ }^{13} \mathrm{C} \mathrm{NMR}(100$ $\left.\mathrm{MHz}, \mathrm{CDCl}_{3}\right)(\delta, \mathrm{ppm}) 173.4,162.6(\mathrm{~d}, J=247.4 \mathrm{~Hz}), 142.0,140.0,138.3(\mathrm{~d}, J=3.3$ Hz), 134.6, 129.2, 128.2, 128.00, 127.99 (d, $J=8.0 \mathrm{~Hz}), 124.1,117.8,115.6$ (d, $J=$ $21.5 \mathrm{~Hz}), 81.9,65.5,62.1,34.2,33.2,{ }^{19} \mathrm{~F} \mathrm{NMR}\left(376 \mathrm{MHz}, \mathrm{CDCl}_{3}\right)(\delta, \mathrm{ppm})-114.01$; HRMS (TOF MS ESI ${ }^{+}$) calculated for $\mathrm{C}_{20} \mathrm{H}_{18} \mathrm{FNO}_{4} \mathrm{SNa}[\mathrm{M}+\mathrm{Na}]^{+}: 410.0833$, found 410.0835; HPLC conditions for determination of enantiomeric excess: Daicel Chiralcel IA, $\lambda=254 \mathrm{~nm}$, hexane : 2-propanol $=90: 10$, flow rate $=1.0 \mathrm{~mL} / \mathrm{min}, t_{\text {minor }}$ $=18.3 \mathrm{~min}, t_{\text {major }}=25.3 \mathrm{~min}$.<smiles>C=C(C[C@@]12COS(=O)(=O)N=C1c1ccccc1CO2)c1ccccc1F</smiles>

(S)-5a-[2-(2-Fluorophenyl)allyl]-5,5a-dihydro-4H,7H-isochromeno[4,3-d][1,2,3]0 xathiazepine 2,2-dioxide (21). Colorless oil. $47.2 \mathrm{mg}, 61 \%$ yield. $92 \%$ ee, $[\alpha]_{\mathrm{D}}{ }^{20}=$ $241^{\circ}(\mathrm{c}=0.1, \mathrm{DCM}) ;{ }^{1} \mathrm{H} \mathrm{NMR}\left(400 \mathrm{MHz}, \mathrm{CDCl}_{3}\right)(\delta, \mathrm{ppm}) 8.26(\mathrm{~d}, J=7.7 \mathrm{~Hz}, 1 \mathrm{H})$, $7.58-7.54(\mathrm{~m}, 1 \mathrm{H}), 7.37(\mathrm{t}, J=7.7 \mathrm{~Hz}, 1 \mathrm{H}), 7.31-7.27(\mathrm{~m}, 1 \mathrm{H}), 7.20-7.16(\mathrm{~m}, 1 \mathrm{H})$, $7.13-7.09(\mathrm{~m}, 2 \mathrm{H}), 7.07-7.03(\mathrm{~m}, 1 \mathrm{H}), 5.72(\mathrm{~s}, 1 \mathrm{H}), 5.40(\mathrm{~s}, 1 \mathrm{H}), 4.94(\mathrm{~d}, J=16.5$ $\mathrm{Hz}, 1 \mathrm{H}), 4.65$ (d, $J=16.5 \mathrm{~Hz}, 1 \mathrm{H}), 4.43-4.40$ (m, 2H), 3.24 (d, $J=16.8 \mathrm{~Hz}, 1 \mathrm{H})$, $3.06(\mathrm{~d}, J=16.8 \mathrm{~Hz}, 1 \mathrm{H}), 2.80-2.72(\mathrm{~m}, 1 \mathrm{H}), 2.43-2.38(\mathrm{~m}, 1 \mathrm{H}) ;{ }^{13} \mathrm{C}$ NMR $(100$ $\left.\mathrm{MHz}, \mathrm{CDCl}_{3}\right)(\delta, \mathrm{ppm}) 173.2,159.5(\mathrm{~d}, J=246.1 \mathrm{~Hz}), 140.1,138.9,134.5,130.4(\mathrm{~d}, J$ $=14.7 \mathrm{~Hz}), 129.9(\mathrm{~d}, J=3.8 \mathrm{~Hz}), 129.7$ (d, $J=8.2 \mathrm{~Hz}), 129.3,128.2,127.9,124.4(\mathrm{~d}$, $J=3.4 \mathrm{~Hz}), 124.1,121.5,116.0(\mathrm{~d}, J=22.4 \mathrm{~Hz}), 81.8,65.5,61.8,34.4,34.1(\mathrm{~d}, J=$ $2.9 \mathrm{~Hz}) ;{ }^{19} \mathrm{~F} \mathrm{NMR}\left(376 \mathrm{MHz}, \mathrm{CDCl}_{3}\right)(\delta, \mathrm{ppm})-115.02$; HRMS (TOF MS ESI ${ }^{+}$) calculated for $\mathrm{C}_{20} \mathrm{H}_{18} \mathrm{FNO}_{4} \mathrm{SNa}[\mathrm{M}+\mathrm{Na}]^{+}: 410.0833$, found 410.0833; HPLC conditions for determination of enantiomeric excess: Daicel Chiralcel IA, $\lambda=254 \mathrm{~nm}$, hexane $:$ 2-propanol $=90: 10$, flow rate $=1.0 \mathrm{~mL} / \mathrm{min}, t_{\text {minor }}=13.5 \mathrm{~min}, t_{\text {major }}=19.5$ $\min$. 
<smiles>C=C(C[C@]12COS(=O)(=O)N=C1c1ccccc1CO2)c1cccc(F)c1</smiles>

(S)-5a-[2-(3-Fluorophenyl)allyl]-5,5a-dihydro-4H,7H-isochromeno[4,3-d] $[1,2,3]$ o xathiazepine 2,2-dioxide (2m). Colorless oil. $42.6 \mathrm{mg}, 55 \%$ yield. $92 \%$ ee, $[\alpha]_{\mathrm{D}}{ }^{20}=$ $168^{\circ}(\mathrm{c}=0.1, \mathrm{DCM}) ;{ }^{1} \mathrm{H} \mathrm{NMR}\left(400 \mathrm{MHz}, \mathrm{CDCl}_{3}\right)(\delta, \mathrm{ppm}) 8.25(\mathrm{~d}, J=8.1 \mathrm{~Hz}, 1 \mathrm{H})$, $7.57(\mathrm{t}, J=7.5 \mathrm{~Hz}, 1 \mathrm{H}), 7.38(\mathrm{t}, J=7.7 \mathrm{~Hz}, 1 \mathrm{H}), 7.32-7.28(\mathrm{~m}, 1 \mathrm{H}), 7.15(\mathrm{~d}, J=7.7$ Hz, 1H), 7.08 (d, J=7.8 Hz, 1H), $7.00-6.97$ (m, 2H), 5.65 (s, 1H), 5.50 (s, 1H), 5.03 (d, $J=16.4 \mathrm{~Hz}, 1 \mathrm{H}), 4.79$ (d, $J=16.4 \mathrm{~Hz}, 1 \mathrm{H}), 4.40-4.36(\mathrm{~m}, 1 \mathrm{H}), 4.27-4.20$ (m, 1H), $3.22(\mathrm{~d}, J=16.9 \mathrm{~Hz}, 1 \mathrm{H}), 3.06(\mathrm{~d}, J=16.8 \mathrm{~Hz}, 1 \mathrm{H}), 2.85-2.76(\mathrm{~m}, 1 \mathrm{H}), 2.42-$ $2.38(\mathrm{~m}, 1 \mathrm{H}) ;{ }^{13} \mathrm{C} \mathrm{NMR}\left(100 \mathrm{MHz}, \mathrm{CDCl}_{3}\right)(\delta, \mathrm{ppm}) 173.2,162.9(\mathrm{~d}, J=246.7 \mathrm{~Hz})$, 144.5 (d, $J=7.3 \mathrm{~Hz}), 142.0,140.0,134.6,130.2$ (d, $J=8.3 \mathrm{~Hz}), 129.3,128.3,128.0$, 124.1, 121.9 (d, $J=2.8 \mathrm{~Hz}), 118.7,115.0(\mathrm{~d}, J=21.1 \mathrm{~Hz}), 113.4(\mathrm{~d}, J=21.9 \mathrm{~Hz})$, 81.9, 65.5, 62.1, 34.2, 32.9; ${ }^{19} \mathrm{~F}$ NMR $\left(376 \mathrm{MHz}, \mathrm{CDCl}_{3}\right)(\delta, \mathrm{ppm})-112.51$; HRMS (TOF MS $\mathrm{ESI}^{+}$) calculated for $\mathrm{C}_{20} \mathrm{H}_{18} \mathrm{FNO}_{4} \mathrm{SNa}[\mathrm{M}+\mathrm{Na}]^{+}: 410.0833$, found 410.0827; HPLC conditions for determination of enantiomeric excess: Daicel Chiralcel IA, $\lambda=254 \mathrm{~nm}$, hexane : 2-propanol $=90: 10$, flow rate $=1.0 \mathrm{~mL} / \mathrm{min}, t_{\text {minor }}$ $=17.8 \mathrm{~min}, t_{\text {major }}=20.9 \mathrm{~min}$.

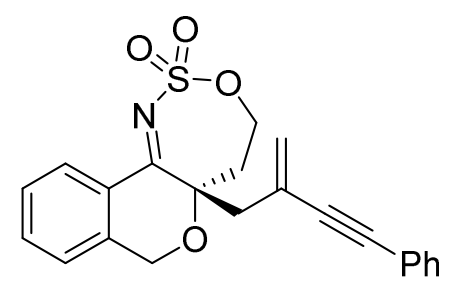

(S)-5a-(2-Methylene-4-phenylbut-3-yn-1-yl)-5,5a-dihydro-4H,7H-isochromeno[4,

3-d][1,2,3]oxathiazepine 2,2-dioxide (2n). Colorless oil. $33.8 \mathrm{mg}, 43 \%$ yield. 91\% ee, $[\alpha]_{\mathrm{D}}{ }^{20}=84^{\mathrm{o}}(\mathrm{c}=0.05, \mathrm{DCM}) ;{ }^{1} \mathrm{H} \mathrm{NMR}\left(400 \mathrm{MHz}, \mathrm{CDCl}_{3}\right)(\delta, \mathrm{ppm}) 8.30(\mathrm{~d}, J=7.9$ Hz, 1H), 7.60 - 7.56 (m, 1H), 7.47 - 7.43 (m, 2H), 7.41 - 7.35 (comp, 4H), 7.19 (d, J $=7.7 \mathrm{~Hz}, 1 \mathrm{H}), 5.76(\mathrm{~s}, 1 \mathrm{H}), 5.63(\mathrm{~s}, 1 \mathrm{H}), 5.40(\mathrm{~d}, J=16.7 \mathrm{~Hz}, 1 \mathrm{H}), 4.89(\mathrm{~d}, J=16.7$ 
$\mathrm{Hz}, 1 \mathrm{H}), 4.80-4.73(\mathrm{~m}, 1 \mathrm{H}), 4.53-4.48(\mathrm{~m}, 1 \mathrm{H}), 3.00$ (q, J = 15.9 Hz, 2H), $2.84-$ $2.75(\mathrm{~m}, 1 \mathrm{H}), 2.60-2.55(\mathrm{~m}, 1 \mathrm{H}) ;{ }^{13} \mathrm{C} \mathrm{NMR}\left(100 \mathrm{MHz}, \mathrm{CDCl}_{3}\right)(\delta, \mathrm{ppm}) 172.9$, $140.0,134.7,131.6,129.3,128.9,128.7,128.2,127.8,127.5,124.9,124.2,122.6$, 90.4, 89.3, 81.0, 65.8, 62.1, 34.9, 34.7; HRMS (TOF MS ESI ${ }^{+}$) calculated for $\mathrm{C}_{22} \mathrm{H}_{19} \mathrm{NO}_{4} \mathrm{SNa}[\mathrm{M}+\mathrm{Na}]^{+}:$416.0927, found 416.0935; HPLC conditions for determination of enantiomeric excess: Daicel Chiralcel IA, $\lambda=254 \mathrm{~nm}$, hexane : 2-propanol $=90: 10$, flow rate $=1.0 \mathrm{~mL} / \mathrm{min}, t_{\text {minor }}=15.2 \mathrm{~min}, t_{\text {major }}=16.0 \mathrm{~min}$.

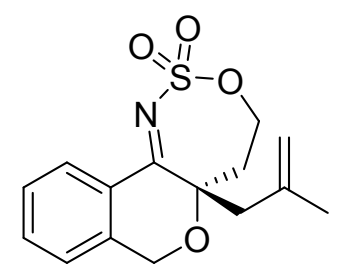

(S)-5a-(2-Methylallyl)-5,5a-dihydro-4H,7H-isochromeno[4,3-d] [1,2,3]oxathiazepi ne 2,2-dioxide (2o). Colorless oil. $44.3 \mathrm{mg}, 72 \%$ yield. $94 \%$ ee, $[\alpha]_{\mathrm{D}}{ }^{20}=531^{\circ}(\mathrm{c}=0.1$, DCM); ${ }^{1} \mathrm{H}$ NMR $\left(400 \mathrm{MHz}, \mathrm{CDCl}_{3}\right)(\delta, \mathrm{ppm}) 8.28(\mathrm{~d}, J=8.1 \mathrm{~Hz}, 1 \mathrm{H}), 7.57(\mathrm{t}, J=7.5$ $\mathrm{Hz}, 1 \mathrm{H}), 7.38$ (t, $J=7.7 \mathrm{~Hz}, 1 \mathrm{H}), 7.18$ (d, $J=7.7 \mathrm{~Hz}, 1 \mathrm{H}), 5.16(\mathrm{~d}, J=17.2 \mathrm{~Hz}, 2 \mathrm{H})$, $5.04(\mathrm{~s}, 1 \mathrm{H}), 4.87(\mathrm{~d}, J=16.5 \mathrm{~Hz}, 1 \mathrm{H}), 4.54-4.42(\mathrm{~m}, 2 \mathrm{H}), 2.84-2.77(\mathrm{~m}, 2 \mathrm{H}), 2.62$ $(\mathrm{d}, J=16.8 \mathrm{~Hz}, 1 \mathrm{H}), 2.36-2.31(\mathrm{~m}, 1 \mathrm{H}), 1.90(\mathrm{~s}, 3 \mathrm{H}) ;{ }^{13} \mathrm{C} \mathrm{NMR}\left(100 \mathrm{MHz}, \mathrm{CDCl}_{3}\right)$

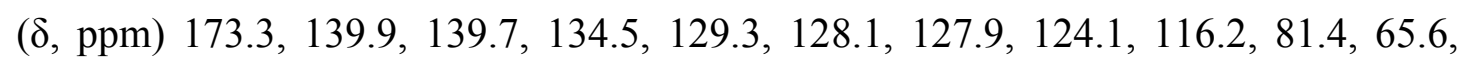
61.9, 34.7, 34.4, 24.1; HRMS (TOF MS ESI ${ }^{+}$) calculated for $\mathrm{C}_{15} \mathrm{H}_{17} \mathrm{NO}_{4} \mathrm{SNa}[\mathrm{M}+$ $\mathrm{Na}]^{+}: 330.0770$, found 330.0783; HPLC conditions for determination of enantiomeric excess: Daicel Chiralcel IA, $\lambda=254 \mathrm{~nm}$, hexane : 2-propanol $=90: 10$, flow rate $=1.0$ $\mathrm{mL} / \mathrm{min}, t_{\mathrm{minor}}=10.8 \mathrm{~min}, t_{\mathrm{major}}=12.8 \mathrm{~min}$.

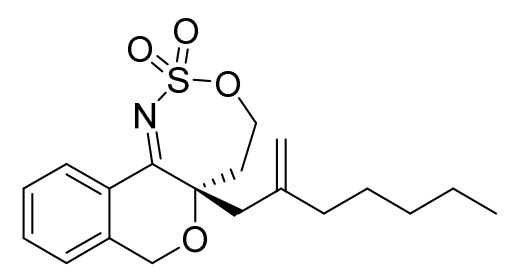

(S)-5a-(2-Methyleneheptyl)-5,5a-dihydro-4H,7H-isochromeno[4,3-d][1,2,3]oxathi azepine 2,2-dioxide (2p). Colorless oil. $47.9 \mathrm{mg}, 66 \%$ yield. $94 \% e e,[\alpha]_{\mathrm{D}}{ }^{20}=315^{\circ}(\mathrm{c}$ $=0.1, \mathrm{DCM}) ;{ }^{1} \mathrm{H}$ NMR $\left(400 \mathrm{MHz}, \mathrm{CDCl}_{3}\right)(\delta, \mathrm{ppm}) 8.28(\mathrm{~d}, J=8.0 \mathrm{~Hz}, 1 \mathrm{H}), 7.59-$ 
$7.55(\mathrm{~m}, 1 \mathrm{H}), 7.38(\mathrm{t}, J=7.7 \mathrm{~Hz}, 1 \mathrm{H}), 7.18(\mathrm{~d}, J=7.6 \mathrm{~Hz}, 1 \mathrm{H}), 5.30(\mathrm{~s}, 1 \mathrm{H}), 5.11(\mathrm{~d}$, $J=16.5 \mathrm{~Hz}, 1 \mathrm{H}), 5.03(\mathrm{~s}, 1 \mathrm{H}), 4.86(\mathrm{~d}, J=16.5 \mathrm{~Hz}, 1 \mathrm{H}), 4.48-4.34$ (m, 2H), $2.88-$ $2.74(\mathrm{~m}, 2 \mathrm{H}), 2.52(\mathrm{~d}, J=17.4 \mathrm{~Hz}, 1 \mathrm{H}), 2.45-2.41(\mathrm{~m}, 1 \mathrm{H}), 2.16-2.02(\mathrm{~m}, 2 \mathrm{H})$, $1.46-1.39(\mathrm{~m}, 2 \mathrm{H}), 1.34-1.26(\mathrm{comp}, 4 \mathrm{H}), 0.88(\mathrm{t}, J=6.9 \mathrm{~Hz}, 3 \mathrm{H}) ;{ }^{13} \mathrm{C} \mathrm{NMR}(100$ $\left.\mathrm{MHz}, \mathrm{CDCl}_{3}\right)(\delta, \mathrm{ppm}) 173.4,143.8,140.1,134.5,129.4,128.2,127.9,124.1,113.7$, 81.6, 65.6, 61.9, 38.1, 34.0, 32.7, 31.7, 27.8, 22.6, 14.2; HRMS (TOF MS ESI ${ }^{+}$) calculated for $\mathrm{C}_{19} \mathrm{H}_{25} \mathrm{NO}_{4} \mathrm{SNa}[\mathrm{M}+\mathrm{Na}]^{+}:$386.1397, found 386.1389; HPLC conditions for determination of enantiomeric excess: Chiralpak IA-3, $\lambda=272 \mathrm{~nm}$, hexane $: 2$-propanol $=90: 10$, flow rate $=1.0 \mathrm{~mL} / \mathrm{min}, t_{\text {minor }}=11.6 \mathrm{~min}, t_{\text {major }}=16.1$ $\min$.

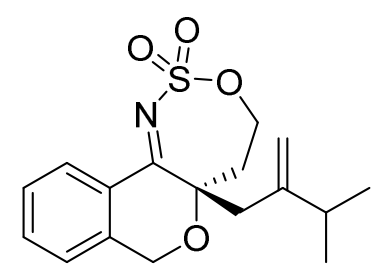

(S)-5a-(3-Methyl-2-methylenebutyl)-5,5a-dihydro-4H,7H-isochromeno[4,3- $d][1,2$, 3]oxathiazepine 2,2-dioxide (2q). Colorless oil. $40.2 \mathrm{mg}, 60 \%$ yield. $95 \%$ ee, $[\alpha]_{\mathrm{D}}{ }^{20}$ $=310^{\circ}(\mathrm{c}=0.1, \mathrm{DCM}){ }^{1} \mathrm{H} \mathrm{NMR}\left(400 \mathrm{MHz}, \mathrm{CDCl}_{3}\right)(\delta, \mathrm{ppm}) 8.28(\mathrm{~d}, J=8.1 \mathrm{~Hz}, 1 \mathrm{H})$, $7.56(\mathrm{t}, J=7.4 \mathrm{~Hz}, 1 \mathrm{H}), 7.38(\mathrm{t}, J=7.7 \mathrm{~Hz}, 1 \mathrm{H}), 7.18(\mathrm{~d}, J=7.7 \mathrm{~Hz}, 1 \mathrm{H}), 5.39$ (s, 1H), $5.09-5.05(\mathrm{~m}, 2 \mathrm{H}), 4.85(\mathrm{~d}, J=16.5 \mathrm{~Hz}, 1 \mathrm{H}), 4.48-4.43(\mathrm{~m}, 1 \mathrm{H}), 4.31-4.24(\mathrm{~m}$, $1 \mathrm{H}), 2.92-2.83(\mathrm{~m}, 1 \mathrm{H}), 2.76(\mathrm{~d}, J=18.0 \mathrm{~Hz}, 1 \mathrm{H}), 2.53-2.43(\mathrm{~m}, 2 \mathrm{H}), 2.31-2.24$ $(\mathrm{m}, 1 \mathrm{H}), 1.04(\mathrm{~d}, J=6.8 \mathrm{~Hz}, 6 \mathrm{H}) ;{ }^{13} \mathrm{C} \mathrm{NMR}\left(100 \mathrm{MHz}, \mathrm{CDCl}_{3}\right)(\delta, \mathrm{ppm}) 173.4,149.7$, 140.3, 134.4, 129.4, 128.1, 127.8, 124.2, 110.5, 81.6, 65.5, 61.9, 36.0, 33.5, 30.4, 21.9, 21.9; HRMS (TOF MS ESI ${ }^{+}$) calculated for $\mathrm{C}_{17} \mathrm{H}_{21} \mathrm{NO}_{4} \mathrm{SNa}[\mathrm{M}+\mathrm{Na}]^{+}: 358.1083$, found 358.1073; HPLC conditions for determination of enantiomeric excess: Chiralpak IA-3, $\lambda=272 \mathrm{~nm}$, hexane : 2-propanol $=90: 10$, flow rate $=1.0 \mathrm{~mL} / \mathrm{min}$, $t_{\text {minor }}=13.6 \mathrm{~min}, t_{\text {major }}=19.8 \mathrm{~min}$. 
<smiles>C=C(Cc1ccccc1)C[C@@]12COS(=O)(=O)N=C1c1ccccc1CO2</smiles>

\section{(S)-5a-(2-Benzylallyl)-5,5a-dihydro-4H,7H-isochromeno[4,3- $d][1,2,3]$ oxathiazepi} ne 2,2-dioxide (2r). Colorless oil. $54.4 \mathrm{mg}, 71 \%$ yield. $93 \%$ ee, $[\alpha]_{\mathrm{D}}{ }^{20}=234^{\circ}(\mathrm{c}=0.1$, DCM); ${ }^{1} \mathrm{H}$ NMR $\left(400 \mathrm{MHz}, \mathrm{CDCl}_{3}\right)(\delta, \mathrm{ppm}) 8.25-8.23(\mathrm{~m}, 1 \mathrm{H}), 7.56$ - $7.52(\mathrm{~m}$, 1H), $7.35(\mathrm{t}, J=7.6 \mathrm{~Hz}, 1 \mathrm{H}), 7.32-7.28(\mathrm{~m}, 2 \mathrm{H}), 7.24-7.21(\mathrm{~m}, 1 \mathrm{H}), 7.18-7.16(\mathrm{~m}$, 2H), $7.12(\mathrm{~d}, J=7.6 \mathrm{~Hz}, 1 \mathrm{H}), 5.44(\mathrm{~s}, 1 \mathrm{H}), 5.08$ (s, 1H), 4.96 (d, $J=16.4 \mathrm{~Hz}, 1 \mathrm{H})$, $4.79(\mathrm{~d}, J=16.5 \mathrm{~Hz}, 1 \mathrm{H}), 4.35-4.30(\mathrm{~m}, 1 \mathrm{H}), 4.13-4.05(\mathrm{~m}, 1 \mathrm{H}), 3.43(\mathrm{dd}, J=39.3$, $14.9 \mathrm{~Hz}, 2 \mathrm{H}), 2.83-2.72(\mathrm{~m}, 2 \mathrm{H}), 2.56(\mathrm{~d}, J=17.5 \mathrm{~Hz}, 1 \mathrm{H}), 2.44-2.38(\mathrm{~m}, 1 \mathrm{H}) ;{ }^{13} \mathrm{C}$ NMR $\left(100 \mathrm{MHz}, \mathrm{CDCl}_{3}\right)(\delta, \mathrm{ppm}) 173.2,143.0,140.1,138.5,134.4,129.2,129.0$, $128.7,128.1,127.8,126.8,124.1,116.4,81.6,65.3,62.0,44.8,33.9,32.1$; HRMS $\left(\right.$ TOF MS ESI ${ }^{+}$) calculated for $\mathrm{C}_{21} \mathrm{H}_{21} \mathrm{NO}_{4} \mathrm{SNa}[\mathrm{M}+\mathrm{Na}]^{+}:$406.1083, found 406.1103; HPLC conditions for determination of enantiomeric excess: Daicel Chiralcel IA, $\lambda=$ $254 \mathrm{~nm}$, hexane $: 2$-propanol $=90: 10$, flow rate $=1.0 \mathrm{~mL} / \mathrm{min}, t_{\text {minor }}=12.5 \mathrm{~min}, t_{\text {major }}$ $=14.3 \mathrm{~min}$.<smiles>C=C(Br)C[C@]12CCOS(=O)(=O)N=C1c1ccccc1CO2</smiles>

\section{(R)-5a-(2-Bromoallyl)-5,5a-dihydro-4H,7H-isochromeno[4,3-d][1,2,3] oxathiazepi}

ne 2,2-dioxide (2s). Colorless oil. $61.0 \mathrm{mg}, 82 \%$ yield. $92 \%$ ee, $[\alpha]_{\mathrm{D}}{ }^{20}=297^{\circ}(\mathrm{c}=0.1$, DCM); ${ }^{1} \mathrm{H}$ NMR $\left(400 \mathrm{MHz}, \mathrm{CDCl}_{3}\right)(\delta, \mathrm{ppm}) 8.24(\mathrm{~d}, J=8.0 \mathrm{~Hz}, 1 \mathrm{H}), 7.60-7.56$ (m, 1H), 7.38 (t, $J=7.7 \mathrm{~Hz}, 1 \mathrm{H}), 7.19$ (d, $J=7.7 \mathrm{~Hz}, 1 \mathrm{H}), 6.07$ (s, 1H), 5.79 (s, 1H), 5.21 $(\mathrm{d}, J=16.7 \mathrm{~Hz}, 1 \mathrm{H}), 4.87(\mathrm{~d}, J=16.7 \mathrm{~Hz}, 1 \mathrm{H}), 4.59-4.47(\mathrm{~m}, 2 \mathrm{H}), 3.16(\mathrm{dd}, J=$ 47.0, 17.5 Hz, 2H), $2.86-2.77(\mathrm{~m}, 1 \mathrm{H}), 2.52-2.47(\mathrm{~m}, 1 \mathrm{H}) ;{ }^{13} \mathrm{C}$ NMR (100 MHz, $\left.\mathrm{CDCl}_{3}\right)(\delta, \mathrm{ppm}) 172.0,139.8,134.8,129.2,128.3,127.4,124.7,124.2,122.4,81.1$, 65.5, 62.0, 37.9, 34.1; HRMS (TOF MS ESI ${ }^{+}$) calculated for $\mathrm{C}_{14} \mathrm{H}_{15} \mathrm{BrNO}_{4} \mathrm{~S}[\mathrm{M}+\mathrm{H}]^{+}$: 
371.9900, found 371.9884; HPLC conditions for determination of enantiomeric excess: Daicel Chiralcel IA, $\lambda=254 \mathrm{~nm}$, hexane : 2-propanol $=90: 10$, flow rate $=1.0$ $\mathrm{mL} / \mathrm{min}, t_{\text {minor }}=14.0 \mathrm{~min}, t_{\text {major }}=16.1 \mathrm{~min}$.

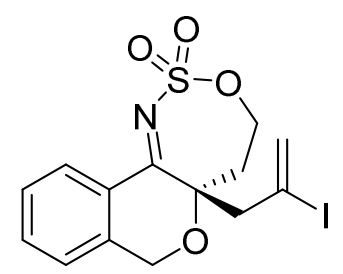

(R)-5a-(2-Iodoallyl)-5,5a-dihydro-4H,7H-isochromeno[4,3- $d][1,2,3]$ oxathiazepine

2,2-dioxide (2t). Colorless oil. $53.6 \mathrm{mg}, 64 \%$ yield. $92 \% e e,[\alpha]_{\mathrm{D}}{ }^{20}=70^{\circ}(\mathrm{c}=0.05$, DCM); ${ }^{1} \mathrm{H}$ NMR (400 MHz, $\left.\mathrm{CDCl}_{3}\right)(\delta, \mathrm{ppm}) 8.28(\mathrm{~d}, J=8.2 \mathrm{~Hz}, 1 \mathrm{H}), 7.61-7.57$ (m, 1H), $7.40(\mathrm{t}, J=7.6 \mathrm{~Hz}, 1 \mathrm{H}), 7.20(\mathrm{~d}, J=7.7 \mathrm{~Hz}, 1 \mathrm{H}), 6.62(\mathrm{~s}, 1 \mathrm{H}), 6.14(\mathrm{t}, J=1.8 \mathrm{~Hz}$, 1H), 5.22 (d, $J=16.7 \mathrm{~Hz}, 1 \mathrm{H}), 4.88$ (d, $J=16.6 \mathrm{~Hz}, 1 \mathrm{H}), 4.53-4.50(\mathrm{~m}, 2 \mathrm{H}), 3.20$ $(\mathrm{dd}, J=44.0,17.6 \mathrm{~Hz}, 2 \mathrm{H}), 2.90-2.81(\mathrm{~m}, 1 \mathrm{H}), 2.53-2.48(\mathrm{~m}, 1 \mathrm{H}) ;{ }^{13} \mathrm{C}$ NMR $(100$ $\left.\mathrm{MHz}, \mathrm{CDCl}_{3}\right)(\delta, \mathrm{ppm}) 171.8,139.8,134.8,131.3,129.4,128.4,127.5,124.3,98.4$, 82.0, 65.6, 62.1, 41.2, 34.0; HRMS (TOF MS ESI ${ }^{+}$) calculated for $\mathrm{C}_{14} \mathrm{H}_{15} \mathrm{INO}_{4} \mathrm{~S}[\mathrm{M}+$ $\mathrm{H}]^{+}:$419.9761, found 419.9757; HPLC conditions for determination of enantiomeric excess: Daicel Chiralcel IA, $\lambda=254 \mathrm{~nm}$, hexane : 2-propanol $=90: 10$, flow rate $=1.0$ $\mathrm{mL} / \mathrm{min}, t_{\text {minor }}=14.5 \mathrm{~min}, t_{\text {major }}=17.9 \mathrm{~min}$.<smiles>CC(=O)CC12CCOS(=O)(=O)N=C1c1ccccc1CO2</smiles>

Methyl (S)-2-[(2,2-dioxido-4,5-dihydro-5aH,7H-isochromeno[4,3-d][1,2,3]oxathiazepin-5a-yl)methyl]acrylate (2u). Colorless oil. $47.0 \mathrm{mg}, 67 \%$ yield. $86 \%$ ee, $[\alpha]_{\mathrm{D}}{ }^{20}=140^{\circ}(\mathrm{c}=0.06, \mathrm{DCM}) ;{ }^{1} \mathrm{H}$ NMR $\left(400 \mathrm{MHz}, \mathrm{CDCl}_{3}\right)(\delta, \mathrm{ppm}) 8.28-8.26(\mathrm{~m}$, 1H), $7.59-7.55(\mathrm{~m}, 1 \mathrm{H}), 7.38(\mathrm{t}, J=7.6 \mathrm{~Hz}, 1 \mathrm{H}), 7.16(\mathrm{~d}, J=7.7 \mathrm{~Hz}, 1 \mathrm{H}), 6.36(\mathrm{~s}$, $1 \mathrm{H}), 6.03(\mathrm{~d}, J=0.8 \mathrm{~Hz}, 1 \mathrm{H}), 5.16(\mathrm{~d}, J=16.5 \mathrm{~Hz}, 1 \mathrm{H}), 4.82(\mathrm{~d}, J=16.5 \mathrm{~Hz}, 1 \mathrm{H})$, $4.47-4.35(\mathrm{~m}, 2 \mathrm{H}), 3.76(\mathrm{~s}, 3 \mathrm{H}), 3.08(\mathrm{dd}, J=40.1,17.4 \mathrm{~Hz}, 2 \mathrm{H}), 2.84-2.75(\mathrm{~m}$, 
1H), $2.31-2.26(\mathrm{~m}, 1 \mathrm{H}) ;{ }^{13} \mathrm{C} \mathrm{NMR}\left(100 \mathrm{MHz}, \mathrm{CDCl}_{3}\right)(\delta, \mathrm{ppm}) 172.9,167.4,140.0$, $134.8,134.6,129.2,128.6,128.2,127.8,124.1,81.3,65.3,62.1,52.5,34.0,29.4$; HRMS (TOF MS ESI ${ }^{+}$) calculated for $\mathrm{C}_{16} \mathrm{H}_{18} \mathrm{NO}_{6} \mathrm{~S}[\mathrm{M}+\mathrm{H}]^{+}: 352.0849$, found 352.0836; HPLC conditions for determination of enantiomeric excess: Daicel Chiralcel IB, $\lambda=254 \mathrm{~nm}$, hexane $: 2$-propanol $=80: 20$, flow rate $=1.0 \mathrm{~mL} / \mathrm{min}, t_{\text {minor }}$ $=11.8 \mathrm{~min}, t_{\text {major }}=13.0 \mathrm{~min}$.<smiles>C=CC(C)(C)[C@@]12CCOS(=O)(=O)N=C1c1ccccc1CO2</smiles>

(R)-5a-(2-Methylbut-3-en-2-yl)-5,5a-dihydro-4H,7H-isochromeno $[4,3-d][1,2,3]$ ox athiazepine 2,2-dioxide (2v). Colorless oil. $20.6 \mathrm{mg}, 32 \%$ yield. $92 \% e e,[\alpha]_{\mathrm{D}}{ }^{20}=$ $218^{\circ}(\mathrm{c}=0.05, \mathrm{DCM}) ;{ }^{1} \mathrm{H} \mathrm{NMR}\left(400 \mathrm{MHz}, \mathrm{CDCl}_{3}\right)(\delta, \mathrm{ppm}) 8.28(\mathrm{~d}, J=8.1 \mathrm{~Hz}, 1 \mathrm{H})$, $7.55(\mathrm{t}, J=7.3 \mathrm{~Hz}, 1 \mathrm{H}), 7.37$ (t, $J=7.7 \mathrm{~Hz}, 1 \mathrm{H}), 7.15(\mathrm{~d}, J=7.7 \mathrm{~Hz}, 1 \mathrm{H}), 5.25-5.24$ (m, 1H), $5.01(\mathrm{~d}, J=16.5 \mathrm{~Hz}, 1 \mathrm{H}), 4.83(\mathrm{~d}, J=16.5 \mathrm{~Hz}, 1 \mathrm{H}), 4.46-4.41(\mathrm{~m}, 1 \mathrm{H})$, $4.26-4.19(\mathrm{~m}, 1 \mathrm{H}), 2.84-2.70(\mathrm{~m}, 2 \mathrm{H}), 2.61(\mathrm{~d}, J=16.6 \mathrm{~Hz}, 1 \mathrm{H}), 2.20-2.16(\mathrm{~m}$, 1H), 1.77 (s, 3H), $1.65(\mathrm{~s}, 3 \mathrm{H}) ;{ }^{13} \mathrm{C}$ NMR (100 MHz, $\left.\mathrm{CDCl}_{3}\right)(\delta, \mathrm{ppm})$ 174.0, 140.0, 137.1, 134.5, 129.1, 128.0, 127.8, 124.1, 115.2, 80.7, 65.4, 61.9, 34.1, 26.9, 26.1, 18.5; HRMS (TOF MS ESI ${ }^{+}$) calculated for $\mathrm{C}_{16} \mathrm{H}_{19} \mathrm{NO}_{4} \mathrm{SNa}[\mathrm{M}+\mathrm{Na}]^{+}: 344.0927$, found 344.0912; HPLC conditions for determination of enantiomeric excess: Daicel Chiralcel IA, $\lambda=254 \mathrm{~nm}$, hexane : 2-propanol $=90: 10$, flow rate $=1.0 \mathrm{~mL} / \mathrm{min}, t_{\text {minor }}$ $=10.8 \mathrm{~min}, t_{\text {major }}=15.0 \mathrm{~min}$.<smiles>C=C[C@H](C)[C@]12CCOS(=O)(=O)N=C1c1ccccc1CO2</smiles>

\section{5a-[(S)-But-3-en-2-yl]-5,5a-dihydro-4H,7H-isochromeno[4,3- $d][1,2,3]$ oxathiazepi} ne 2,2-dioxide (2w). Colorless oil. $34.4 \mathrm{mg}, 56 \%$ yield. $90 \%$ ee, $[\alpha]_{\mathrm{D}}{ }^{20}=66^{\circ}(\mathrm{c}=0.1$, 
DCM); ${ }^{1} \mathrm{H}$ NMR $\left(400 \mathrm{MHz}, \mathrm{CDCl}_{3}\right)(\delta, \mathrm{ppm}) 8.08(\mathrm{~d}, J=7.9 \mathrm{~Hz}, 1 \mathrm{H}), 7.56-7.52(\mathrm{~m}$, 1H), $7.37(\mathrm{t}, J=7.6 \mathrm{~Hz}, 1 \mathrm{H}), 7.16(\mathrm{~d}, J=7.6 \mathrm{~Hz}, 1 \mathrm{H}), 5.88-5.79(\mathrm{~m}, 1 \mathrm{H}), 5.10(\mathrm{dd}$, $J=27.8,13.7 \mathrm{~Hz}, 2 \mathrm{H}), 4.88(\mathrm{q}, J=15.8 \mathrm{~Hz}, 2 \mathrm{H}), 4.55-4.48(\mathrm{~m}, 1 \mathrm{H}), 4.43-4.38(\mathrm{~m}$, $1 \mathrm{H}), 3.26(\mathrm{p}, J=7.0 \mathrm{~Hz}, 1 \mathrm{H}), 2.76-2.68(\mathrm{~m}, 1 \mathrm{H}), 2.24-2.19(\mathrm{~m}, 1 \mathrm{H}), 1.01(\mathrm{~d}, J=$ $6.9 \mathrm{~Hz}, 3 \mathrm{H}) ;{ }^{13} \mathrm{C} \mathrm{NMR}\left(100 \mathrm{MHz}, \mathrm{CDCl}_{3}\right)(\delta, \mathrm{ppm}) 175.8,139.9,137.4,134.1,130.7$, 128.3, 128.1, 124.0, 117.7, 83.8, 65.5, 62.7, 41.5, 32.1, 15.2; HRMS (TOF MS ESI ${ }^{+}$) calculated for $\mathrm{C}_{15} \mathrm{H}_{17} \mathrm{NO}_{4} \mathrm{SNa}[\mathrm{M}+\mathrm{Na}]^{+}$: 330.0770, found 330.0775; HPLC conditions for determination of enantiomeric excess: Daicel Chiralcel IA, $\lambda=254 \mathrm{~nm}$, hexane $: 2$-propanol $=90: 10$, flow rate $=1.0 \mathrm{~mL} / \mathrm{min}, t_{\text {minor }}=11.9 \mathrm{~min}, t_{\text {major }}=13.0$ $\min$.

\section{General Procedure for the Intermolecular Asymmetric Cascade Reaction :}

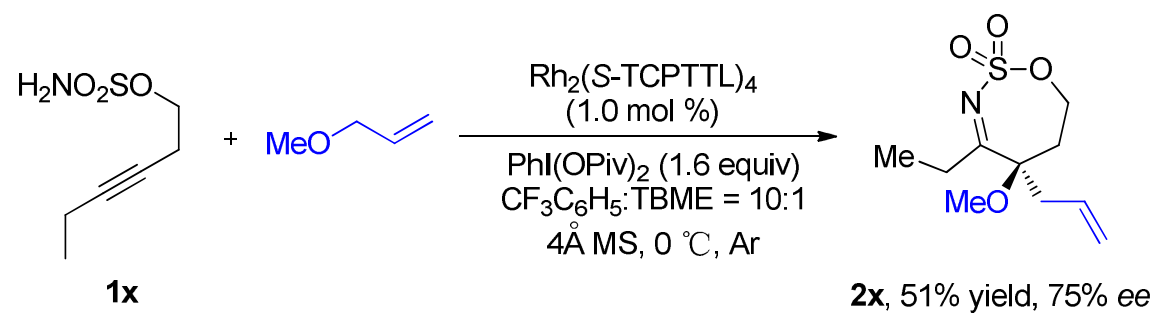

To a $10-\mathrm{mL}$ oven-dried vial containing a magnetic stirring bar, $\mathrm{Rh}_{2}(\mathrm{~S} \text {-TCPTTL })_{4}(3.6$ $\mathrm{mg}, 1.0 \mathrm{~mol} \%)$, and $4 \AA \mathrm{MS}(100 \mathrm{mg})$ in mixed solvent $\left(\mathrm{C}_{6} \mathrm{H}_{5} \mathrm{CF}_{3}: \mathrm{TBME}=10: 1,0.5\right.$ $\mathrm{mL}$ ), was added as a solution of sulfamate ester 1x (36.0 mg, $0.2 \mathrm{mmol}), \mathrm{PhI}(\mathrm{OPiv}) 2$ (128.0 mg, 1.6 equiv), and methyl allyl ether $(112.0 \mu \mathrm{L}, 6.0$ equiv) in the same mixed solvent $(1.0 \mathrm{~mL})$ via a syringe pump over $1.5 \mathrm{~h}$ under argon atmosphere at $0{ }^{\circ} \mathrm{C}$. After addition, the reaction mixture was stirred under these conditions until consumption of the material (monitored by TLC, about 4 days). Then the reaction mixture was purified by column chromatography on silica gel without any additional treatment (Hexanes : EtOAc $=4: 1$ to $2: 1$ ) to give the pure products $\mathbf{2} \mathbf{x}$ as colorless oil, ${ }^{1} 25.2 \mathrm{mg}$, $51 \%$ yield. $75 \%$ ee, $[\alpha]_{\mathrm{D}}{ }^{20}=9^{\circ}(\mathrm{c}=0.15, \mathrm{DCM}) .{ }^{1} \mathrm{H} \mathrm{NMR}\left(400 \mathrm{MHz}, \mathrm{CDCl}_{3}\right)(\delta, \mathrm{ppm})$ $5.96-5.85(\mathrm{~m}, 1 \mathrm{H}), 5.22-5.18(\mathrm{~m}, 2 \mathrm{H}), 4.43-4.39(\mathrm{~m}, 1 \mathrm{H}), 4.32-4.25(\mathrm{~m}, 1 \mathrm{H})$, $3.26(\mathrm{~s}, 3 \mathrm{H}), 2.94-2.73(\mathrm{~m}, 2 \mathrm{H}), 2.64-2.47$ (comp, 3H), $2.01-1.96(\mathrm{~m}, 1 \mathrm{H}), 1.12(\mathrm{t}$, $J=7.1 \mathrm{~Hz}, 3 \mathrm{H}) ;{ }^{13} \mathrm{C} \mathrm{NMR}\left(100 \mathrm{MHz}, \mathrm{CDCl}_{3}\right)(\delta, \mathrm{ppm}) 193.2,131.2,120.1,84.6$, 
64.6, 52.7, 39.6, 31.3, 30.1, 10.1; HPLC conditions for determination of enantiomeric excess: Daicel Chiralcel IA, $\lambda=254 \mathrm{~nm}$, hexane : 2-propanol $=90: 10$, flow rate $=1.0$ $\mathrm{mL} / \mathrm{min}, t_{\mathrm{minor}}=6.7 \mathrm{~min}, t_{\mathrm{major}}=7.4 \mathrm{~min}$.

\section{General Procedure of the Scale Up Reaction for the Synthesis of 2s}

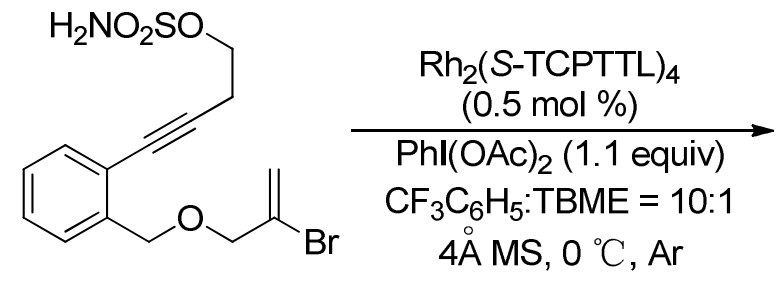

$1 \mathrm{~s}, 5.0 \mathrm{mmol}, 1.87 \mathrm{~g}$<smiles>C=C(Br)C[C@]12CCOS(=O)(=O)N=C1c1ccccc1CO2</smiles>

2s, $75 \%$ yield, $1.40 \mathrm{~g}, 91 \%$ ee

To a 50-mL oven-dried vial containing a magnetic stirring bar, $\mathrm{Rh}_{2}(S \text {-TCPTTL })_{4}$

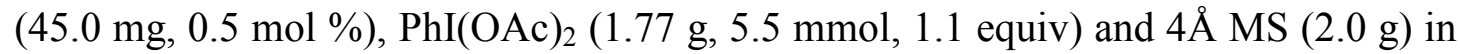
mixed solvent $\left(\mathrm{C}_{6} \mathrm{H}_{5} \mathrm{CF}_{3}: \mathrm{TBME}=10: 1,10 \mathrm{~mL}\right)$, was added as a solution of sulfamate ester $1 \mathrm{~s}(1.87 \mathrm{~g}, 5.0 \mathrm{mmol})$ in the same solvent $(20 \mathrm{~mL})$ via a syringe pump over $2 \mathrm{~h}$ under argon atmosphere at $0{ }^{\circ} \mathrm{C}$. After addition, the reaction mixture was stirred at $0{ }^{\circ} \mathrm{C}$ for additional $36 \mathrm{~h}$. Then the solvent was evaporated in vacuo, the residue was purified by column chromatography on silica gel (Hexanes : EtOAc $=4: 1$ to 2:1) to give $1.40 \mathrm{~g}$ of pure $2 \mathrm{~s}$ in $75 \%$ yield with $91 \%$ ee.

\section{General Procedure for the Synthesis of 3}<smiles>C=C(Br)CC12CCOS(=O)(=O)N=C1c1ccccc1CO2</smiles>

2s, $91 \%$ ee

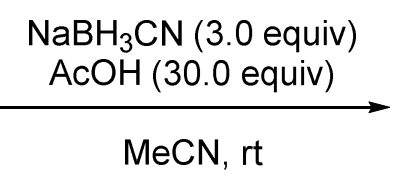

$3,91 \%$ yield, $91 \%$ ee

To a stirred solution of $2 \mathrm{~s}$ (91\% ee, $74.4 \mathrm{mg}, 0.2 \mathrm{mmol}, 1.0$ equiv) in anhydrous MeCN (1.0 mL), was added $\mathrm{NaBH}_{3} \mathrm{CN}(37.7 \mathrm{mg}, 0.6 \mathrm{mmol}, 3.0$ equiv) and $\mathrm{AcOH}$ $\left(0.36 \mathrm{~g}, 6.0 \mathrm{mmol}, 30.0\right.$ equiv) in sequence at $0{ }^{\circ} \mathrm{C}$. The reaction mixture was warmed 
to room temperature and stirred for additional $14 \mathrm{~h}$ under these conditions. The mixture was quenched with saturated aqueous $\mathrm{NaHCO}_{3}$ solution $(10 \mathrm{~mL})$, and extracted with $\mathrm{Et}_{2} \mathrm{O}(2 \mathrm{X} 10 \mathrm{~mL})$. The combined extract was washed with brine, dried with $\mathrm{Na}_{2} \mathrm{SO}_{4}$, and then concentrated under reduced pressure after filtration. The crude product was purified by flash chromatography on silica gel (Hexanes : EtOAc $=4: 1$ to 2:1) to give pure product 3 as white solid, $\mathrm{mp}=140-141{ }^{\circ} \mathrm{C} .68 .1 \mathrm{mg}, 91 \%$ yield. $91 \% e e,[\alpha]_{\mathrm{D}}{ }^{20}=144^{\circ}(\mathrm{c}=0.07, \mathrm{DCM}) .{ }^{1} \mathrm{H} \mathrm{NMR}\left(400 \mathrm{MHz}, \mathrm{CDCl}_{3}\right)(\delta, \mathrm{ppm}) 7.60-$ $7.58(\mathrm{~m}, 1 \mathrm{H}), 7.32-7.30(\mathrm{~m}, 2 \mathrm{H}), 7.06-7.04(\mathrm{~m}, 1 \mathrm{H}), 5.92(\mathrm{~s}, 1 \mathrm{H}), 5.73(\mathrm{~d}, J=1.2$ $\mathrm{Hz}, 1 \mathrm{H}), 5.01-4.97$ (m, 2H), 4.83 (d, $J=16.3 \mathrm{~Hz}, 1 \mathrm{H}), 4.75$ (d, $J=10.7 \mathrm{~Hz}, 1 \mathrm{H})$, $4.53-4.47(\mathrm{~m}, 1 \mathrm{H}), 4.43-4.38(\mathrm{~m}, 1 \mathrm{H}), 2.86-2.75(\mathrm{~m}, 2 \mathrm{H}), 2.647-2.59(\mathrm{~m}, 1 \mathrm{H})$, 2.26 - $2.19(\mathrm{~m}, 1 \mathrm{H}) ;{ }^{13} \mathrm{C} \mathrm{NMR}\left(100 \mathrm{MHz}, \mathrm{CDCl}_{3}\right)(\delta, \mathrm{ppm}) 133.8,129.8,128.2$, 127.7, 126.4, 125.8, 124.0, 121.9, 75.9, 74.4, 62.9, 56.7, 37.9, 36.4; HRMS (TOF MS $\mathrm{ESI}^{+}$) calculated for $\mathrm{C}_{14} \mathrm{H}_{16} \mathrm{BrNO}_{4} \mathrm{SNa}[\mathrm{M}+\mathrm{Na}]^{+}$: 395.9876, found 395.9866; HPLC conditions for determination of enantiomeric excess: Daicel Chiralcel IA, $\lambda=254 \mathrm{~nm}$, hexane $:$ 2-propanol $=90: 10$, flow rate $=1.0 \mathrm{~mL} / \mathrm{min}, t_{\text {minor }}=12.4 \mathrm{~min}, t_{\text {major }}=9.9$ $\min$.

\section{General Procedure for the Synthesis of 4}<smiles>C=C(Br)C[C@]12CCOS(=O)(=O)N[C@H]1c1ccccc1CO2</smiles>

3, $91 \%$ ee

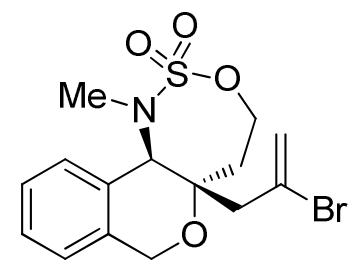

4, $78 \%$ yield, $91 \%$ ee

To a solution of 3 (91\% ee, $86.0 \mathrm{mg}, 0.23 \mathrm{mmol}, 1.0$ equiv) in DMF (2.0 mL) was added MeI (28.6 $\mu \mathrm{L}, 0.46 \mathrm{mmol}, 2.0$ equiv), $\mathrm{K}_{2} \mathrm{CO}_{3}$ (48.0 mg, $0.35 \mathrm{mmol}, 1.5$ equiv), and $n$ - $\mathrm{Bu}_{4} \mathrm{NI}\left(6.3 \mathrm{mg}, 0.023 \mathrm{mmol}, 0.1\right.$ equiv) in sequence at $0{ }^{\circ} \mathrm{C}$, and the mixture was stirred at room temperature for $12 \mathrm{~h}$. The reaction was quenched with $\mathrm{H}_{2} \mathrm{O}(15$ $\mathrm{mL})$ and extracted with ethyl acetate $(3 \mathrm{X} 10 \mathrm{~mL})$. The combined organic layer was washed successively with water and brine, dried over anhydrous $\mathrm{Na}_{2} \mathrm{SO}_{4}$, and then 
concentrated under reduced pressure after filtration. The crude product was purified by flash chromatography on silica gel (Hexanes : EtOAc $=10: 1$ to $5: 1$ ) to give pure product 4 as white solid, $\mathrm{mp}=208-209{ }^{\circ} \mathrm{C} .69 .6 \mathrm{mg}, 78 \%$ yield. $91 \%$ ee, $[\alpha]_{\mathrm{D}}{ }^{20}=$ $164^{\circ}(\mathrm{c}=0.05, \mathrm{DCM}) .{ }^{1} \mathrm{H}$ NMR $\left(400 \mathrm{MHz}, \mathrm{CDCl}_{3}\right)(\delta, \mathrm{ppm}) 7.33-7.25$ (comp, 3H), $7.07(\mathrm{~d}, J=7.0 \mathrm{~Hz}, 1 \mathrm{H}), 6.01(\mathrm{~s}, 1 \mathrm{H}), 5.75-5.74(\mathrm{~m}, 1 \mathrm{H}), 5.17(\mathrm{~s}, 1 \mathrm{H}), 4.96$ (d, $J=$ $16.2 \mathrm{~Hz}, 1 \mathrm{H}), 4.68(\mathrm{~d}, J=16.2 \mathrm{~Hz}, 1 \mathrm{H}), 4.49-4.36(\mathrm{~m}, 2 \mathrm{H}), 3.03-2.98(\mathrm{~m}, 1 \mathrm{H})$, $2.84(\mathrm{~s}, 3 \mathrm{H}), 2.74(\mathrm{~d}, J=16.9 \mathrm{~Hz}, 1 \mathrm{H}), 2.61-2.56(\mathrm{~m}, 1 \mathrm{H}), 2.25-2.16(\mathrm{~m}, 1 \mathrm{H}) ;{ }^{13} \mathrm{C}$ NMR (100 MHz, $\left.\mathrm{CDCl}_{3}\right)(\delta, \mathrm{ppm}) 134.9,128.0,127.9,127.6,127.5,125.9,124.5$, 121.5, 76.8, 67.5, 62.3, 60.5, 38.2, 37.8, 34.3; HRMS (TOF MS ESI ${ }^{+}$) calculated for $\mathrm{C}_{15} \mathrm{H}_{19} \mathrm{BrNO}_{4} \mathrm{~S}[\mathrm{M}+\mathrm{H}]^{+}:$388.0213, found 388.0192; HPLC conditions for determination of enantiomeric excess: Daicel Chiralcel IA, $\lambda=254 \mathrm{~nm}$, hexane : 2-propanol $=90: 10$, flow rate $=1.0 \mathrm{~mL} / \mathrm{min}, t_{\text {minor }}=15.5 \mathrm{~min}, t_{\text {major }}=10.1 \mathrm{~min}$.

\section{General Procedure for the Synthesis of 5}

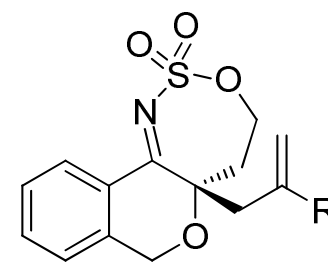

2s, $\mathrm{R}=\mathrm{Br}, 91 \%$ ee

2q, $\mathrm{R}=i \mathrm{Pr}, 95 \%$ ee

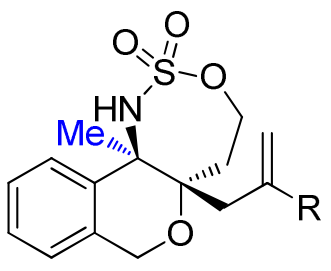

5s, $\mathrm{R}=\mathrm{Br}, 90 \%$ yield, $92 \%$ ee

$5 q, \mathrm{R}=i \mathrm{Pr}, 92 \%$ yield, $95 \%$ ee

To a stirred solution of 2 (0.3 mmol, 1.0 equiv) in anhydrous THF (2.0 mL), was added methyl magnesium bromide (3.0 $\mathrm{M}$ in 2-methyl-THF, $0.3 \mathrm{~mL}, 0.9 \mathrm{mmol})$ drop wise under argon atmosphere at $0{ }^{\circ} \mathrm{C}$. After addition, the reaction mixture was stirred under these conditions until consumption of the material (monitored by TLC, about 2 h). The reaction mixture was quenched with saturated aqueous $\mathrm{NH}_{4} \mathrm{Cl}$ solution $(10$ $\mathrm{mL})$, and extracted with $\mathrm{Et}_{2} \mathrm{O}(3 \mathrm{X} 10 \mathrm{~mL})$. The combined extract was washed with brine, dried with $\mathrm{Na}_{2} \mathrm{SO}_{4}$, and then concentrated under reduced pressure after filtration. The crude product was purified by flash chromatography on silica gel (Hexanes : EtOAc $=5: 1$ to $4: 1$ ) to give pure product 5 in high yields. 


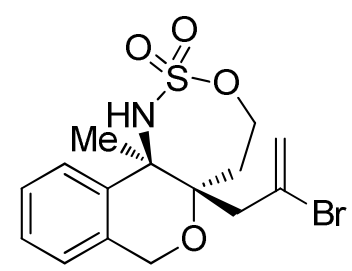

(5aR,11bR)-5a-(2-bromoallyl)-11b-methyl-1,4,5,5a,7,11b-hexahydroisochromeno $[4,3-d][1,2,3]$ oxathiazepine 2,2-dioxide (5s). Colorless solid, $\mathrm{mp}=88-89^{\circ} \mathrm{C} .104 .8$ mg, $90 \%$ yield. $92 \% e e,[\alpha]_{\mathrm{D}}{ }^{20}=114^{\circ}(\mathrm{c}=0.1, \mathrm{DCM}) .{ }^{1} \mathrm{H} \mathrm{NMR}\left(400 \mathrm{MHz}, \mathrm{CDCl}_{3}\right)(\delta$, ppm) $7.63-7.61(\mathrm{~m}, 1 \mathrm{H}), 7.31-7.25(\mathrm{~m}, 2 \mathrm{H}), 7.05-7.03(\mathrm{~m}, 1 \mathrm{H}), 5.83(\mathrm{~s}, 1 \mathrm{H}), 5.71$ (s, 1H), $5.12(\mathrm{~s}, 1 \mathrm{H}), 5.05(\mathrm{~d}, J=16.5 \mathrm{~Hz}, 1 \mathrm{H}), 4.89$ (d, $J=16.5 \mathrm{~Hz}, 1 \mathrm{H}), 4.47-4.34$ (m, 2H), $2.92-2.74(\mathrm{~m}, 2 \mathrm{H}), 2.42-2.40(\mathrm{~m}, 2 \mathrm{H}), 1.78(\mathrm{~s}, 3 \mathrm{H}) ;{ }^{13} \mathrm{C} \mathrm{NMR}(100 \mathrm{MHz}$, $\left.\mathrm{CDCl}_{3}\right)(\delta, \mathrm{ppm}) 138.1,132.5,127.9,127.2,124.2,124.1,122.0,78.6,67.0,63.2$, 60.4, 38.7, 33.0, 23.6; HRMS (TOF MS ESI ${ }^{+}$) calculated for $\mathrm{C}_{15} \mathrm{H}_{19} \mathrm{BrNO}_{4} \mathrm{~S}[\mathrm{M}+\mathrm{H}]^{+}$: 388.0213, found 388.0218; HPLC conditions for determination of enantiomeric excess: Daicel Chiralcel IA, $\lambda=254 \mathrm{~nm}$, hexane : 2-propanol $=90: 10$, flow rate $=1.0$ $\mathrm{mL} / \mathrm{min}, t_{\mathrm{minor}}=10.0 \mathrm{~min}, t_{\mathrm{major}}=7.5 \mathrm{~min}$.

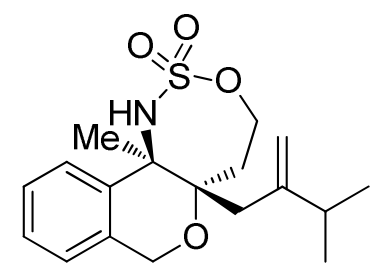

(5aS,11bR)-11b-methyl-5a-(3-methyl-2-methylenebutyl)-1,4,5,5a,7,11b-hexahydr oisochromeno[4,3-d][1,2,3]oxathiazepine 2,2-dioxide (5q). Colorless oil, $97.0 \mathrm{mg}$, $92 \%$ yield. $95 \% e e,[\alpha]_{\mathrm{D}}{ }^{20}=55^{\circ}(\mathrm{c}=0.09, \mathrm{DCM}) .{ }^{1} \mathrm{H} \mathrm{NMR}\left(400 \mathrm{MHz}, \mathrm{CDCl}_{3}\right)(\delta$, ppm) $7.65-7.63(\mathrm{~m}, 1 \mathrm{H}), 7.31-7.23(\mathrm{~m}, 2 \mathrm{H}), 7.01(\mathrm{~d}, J=7.1 \mathrm{~Hz}, 1 \mathrm{H}), 5.09$ (d, $J=$ $8.0 \mathrm{~Hz}, 2 \mathrm{H}), 5.00(\mathrm{~s}, 1 \mathrm{H}), 4.87(\mathrm{q}, J=16.3 \mathrm{~Hz}, 2 \mathrm{H}), 4.43-4.36(\mathrm{~m}, 1 \mathrm{H}), 4.33-4.26$ $(\mathrm{m}, 1 \mathrm{H}), 2.34-2.16$ (comp, 5H), $1.79(\mathrm{~s}, 3 \mathrm{H}), 1.00(\mathrm{dd}, J=18.3,6.8 \mathrm{~Hz}, 6 \mathrm{H}) ;{ }^{13} \mathrm{C}$ NMR (100 MHz, $\left.\mathrm{CDCl}_{3}\right)(\delta, \mathrm{ppm}) 151.2,138.9,133.2,127.6,124.5,123.8,111.0$, 78.7, 67.0, 63.2, 60.7, 34.4, 32.9, 31.6, 23.5, 22.6, 21.7; HRMS (TOF MS ESI ${ }^{+}$) calculated for $\mathrm{C}_{18} \mathrm{H}_{25} \mathrm{NO}_{4} \mathrm{SNa}[\mathrm{M}+\mathrm{Na}]^{+}:$374.1397, found 374.1407; HPLC 
conditions for determination of enantiomeric excess: Daicel Chiralcel IA, $\lambda=254 \mathrm{~nm}$, hexane : 2 -propanol $=90: 10$, flow rate $=1.0 \mathrm{~mL} / \mathrm{min}, t_{\mathrm{minor}}=6.2 \mathrm{~min}, t_{\mathrm{major}}=5.4 \mathrm{~min}$.

\section{General Procedure for the Synthesis of 6}<smiles>C=CC[C@]12CCOS(=O)(=O)N=C1c1ccccc1O2</smiles>

2d, $>99 \%$ ee
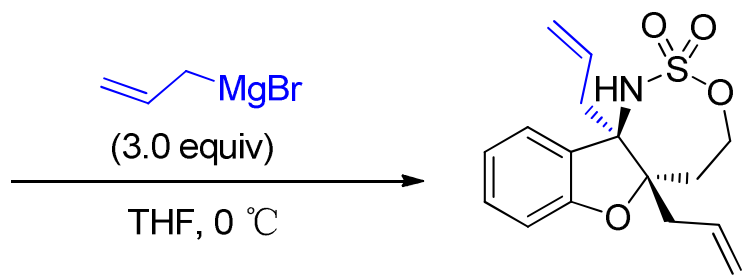

$6,84 \%$ yield, $94 \%$ ee

To a stirred solution of $\mathbf{2 d}$ ( $>99 \%$ ee, $27.9 \mathrm{mg}, 0.1 \mathrm{mmol}, 1.0$ equiv) in anhydrous THF (1.0 mL), was added allyl magnesium bromide (1.0 $\mathrm{M}$ in Et $2 \mathrm{O}, 0.3 \mathrm{~mL}, 0.3$ mmol) drop wise under argon atmosphere at $0{ }^{\circ} \mathrm{C}$. After addition, the reaction mixture was stirred under these conditions until consumption of the material (monitored by TLC, about $2 \mathrm{~h}$ ). The reaction mixture was quenched with saturated aqueous $\mathrm{NH}_{4} \mathrm{Cl}$ solution $(10 \mathrm{~mL})$, and extracted with $\mathrm{Et}_{2} \mathrm{O}(3 \mathrm{X} 10 \mathrm{~mL})$. The combined extract was washed with brine, dried with $\mathrm{Na}_{2} \mathrm{SO}_{4}$, and then concentrated under reduced pressure after filtration. The crude product was purified by flash chromatography on silica gel (Hexanes : EtOAc $=5: 1$ to 4:1) to give pure product 6 as colorless oil. $27.0 \mathrm{mg}, 84 \%$ yield. $94 \% e e,[\alpha]_{\mathrm{D}}{ }^{20}=39^{\circ}(\mathrm{c}=0.1, \mathrm{DCM}) .{ }^{1} \mathrm{H} \mathrm{NMR}\left(400 \mathrm{MHz}, \mathrm{CDCl}_{3}\right)(\delta, \mathrm{ppm})$ $7.23-7.19(\mathrm{~m}, 1 \mathrm{H}), 7.08-7.06(\mathrm{~m}, 1 \mathrm{H}), 6.91(\mathrm{t}, J=7.3 \mathrm{~Hz}, 1 \mathrm{H}), 6.81(\mathrm{~d}, J=8.0 \mathrm{~Hz}$, 1H), $5.86-5.68(\mathrm{~m}, 2 \mathrm{H}), 5.19-5.06(\mathrm{comp}, 4 \mathrm{H}), 4.86-4.82(\mathrm{~m}, 1 \mathrm{H}), 4.66-4.59(\mathrm{~m}$, $2 \mathrm{H}), 3.30-3.25(\mathrm{~m}, 1 \mathrm{H}), 2.79-2.73(\mathrm{~m}, 1 \mathrm{H}), 2.62-2.51(\mathrm{~m}, 2 \mathrm{H}), 2.43-2.32(\mathrm{~m}$, $2 \mathrm{H}) ;{ }^{13} \mathrm{C} \mathrm{NMR}\left(100 \mathrm{MHz}, \mathrm{CDCl}_{3}\right)(\delta, \mathrm{ppm}) 156.1,132.4,131.7,130.6,129.9,124.4$, 121.1, 120.3, 119.6, 111.6, 93.5, 70.4, 68.6, 38.1, 35.8, 29.4; HRMS (TOF MS ESI') calculated for $\mathrm{C}_{16} \mathrm{H}_{19} \mathrm{NO}_{4} \mathrm{SNa}[\mathrm{M}+\mathrm{Na}]^{+}$: 344.0927, found 344.0934; HPLC conditions for determination of enantiomeric excess: Daicel Chiralcel IA, $\lambda=254 \mathrm{~nm}$, hexane $: 2$-propanol $=90: 10$, flow rate $=1.0 \mathrm{~mL} / \mathrm{min}, t_{\text {minor }}=7.9 \mathrm{~min}, t_{\mathrm{major}}=7.3 \mathrm{~min}$. 


\section{D-noe NMR Analysis of 2w, 3, and 5s}
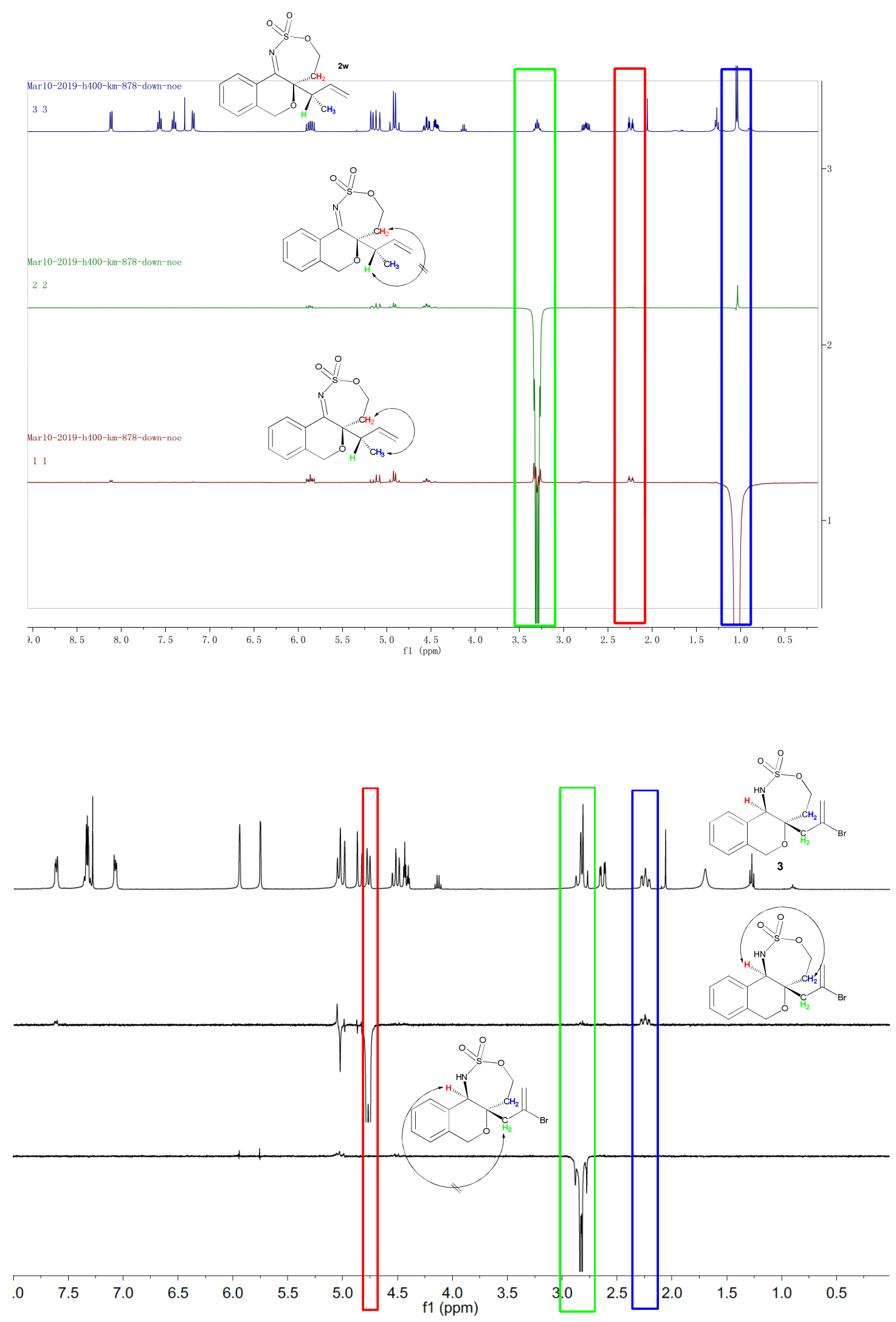


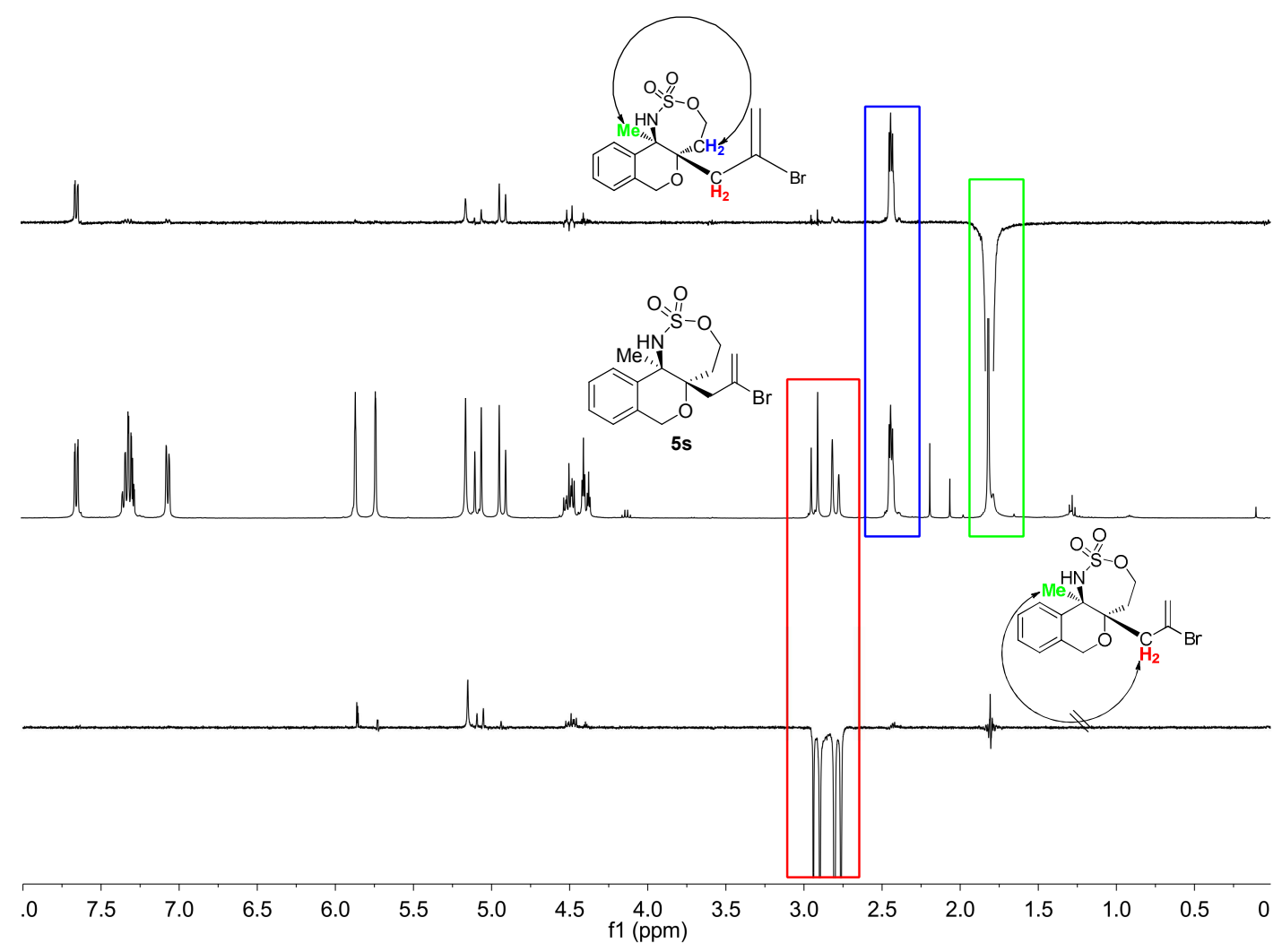

\section{References:}

1 (a) Thornton, A. R.; Blakey, S. B. J. Am. Chem. Soc. 2008, 130, 5020. (b) Mace, N.; Thornton, A. R.; Blakey, S. B. Angew. Chem. Int. Ed. 2013, 52, 5836. 


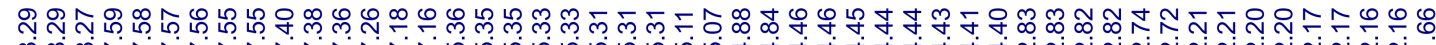

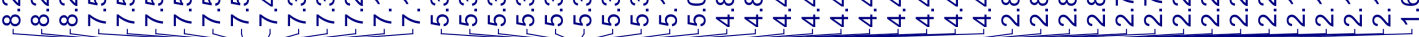
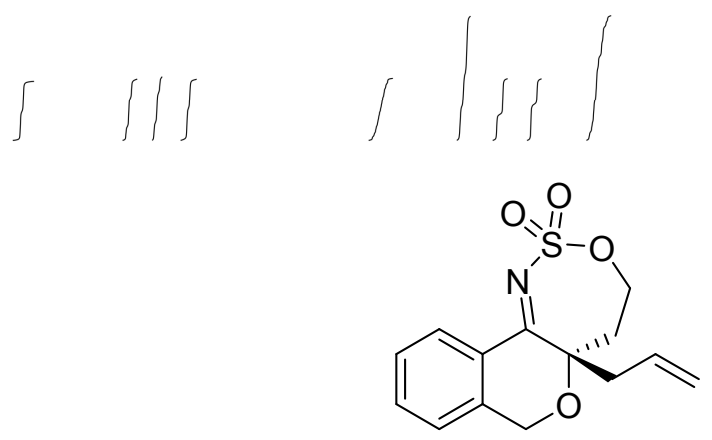

$2 a$
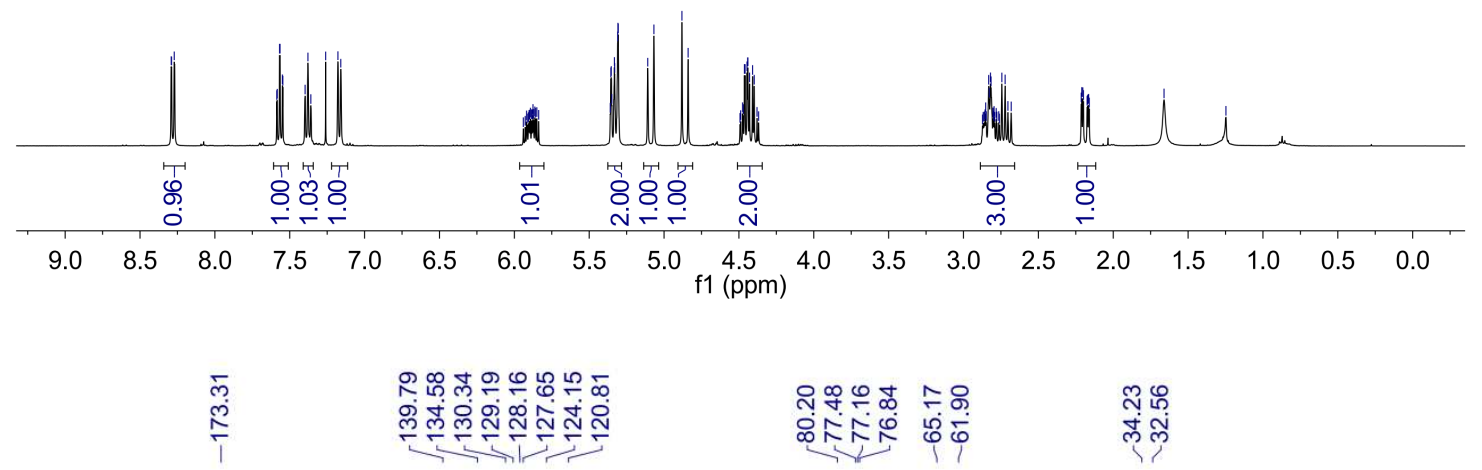

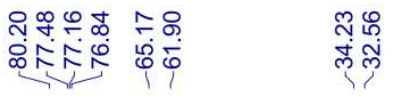

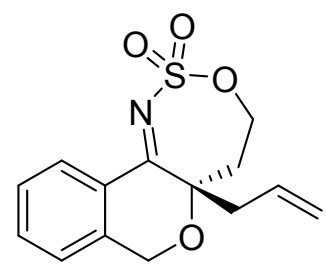

$2 a$

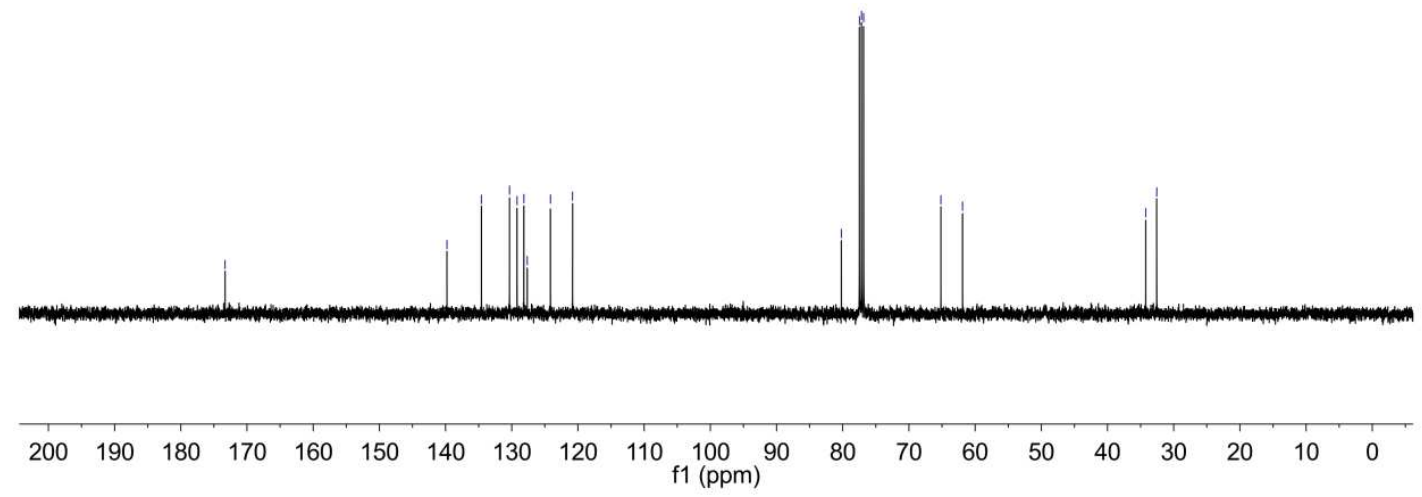



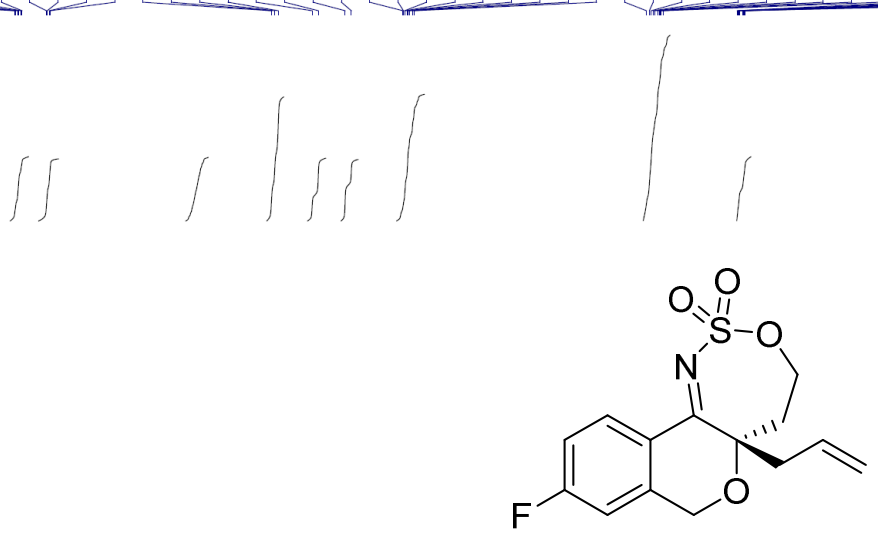

2b
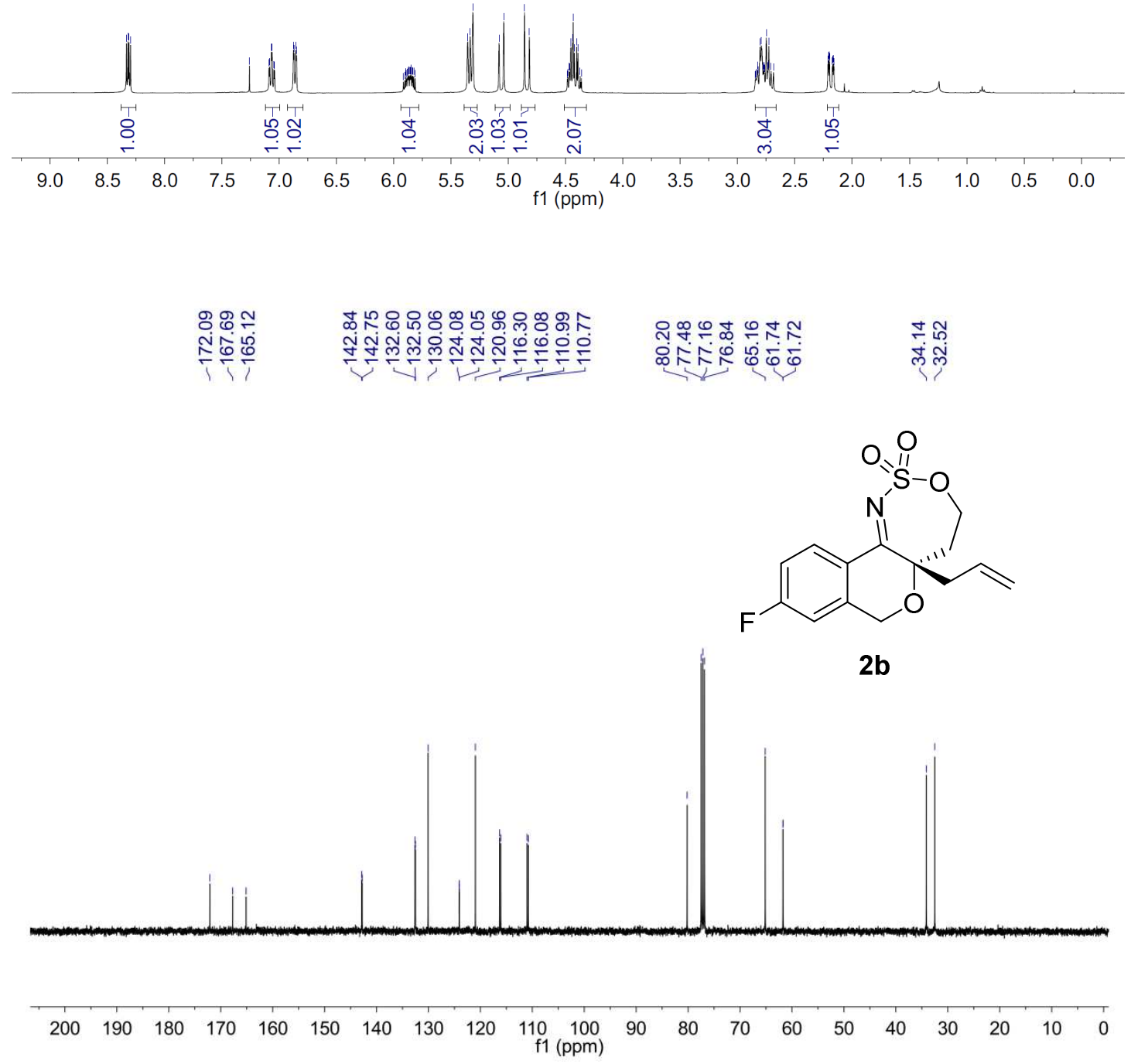


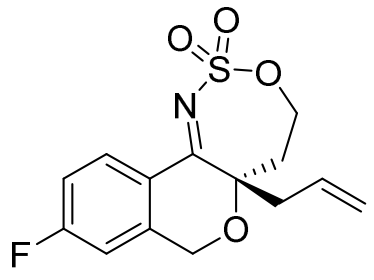

2b

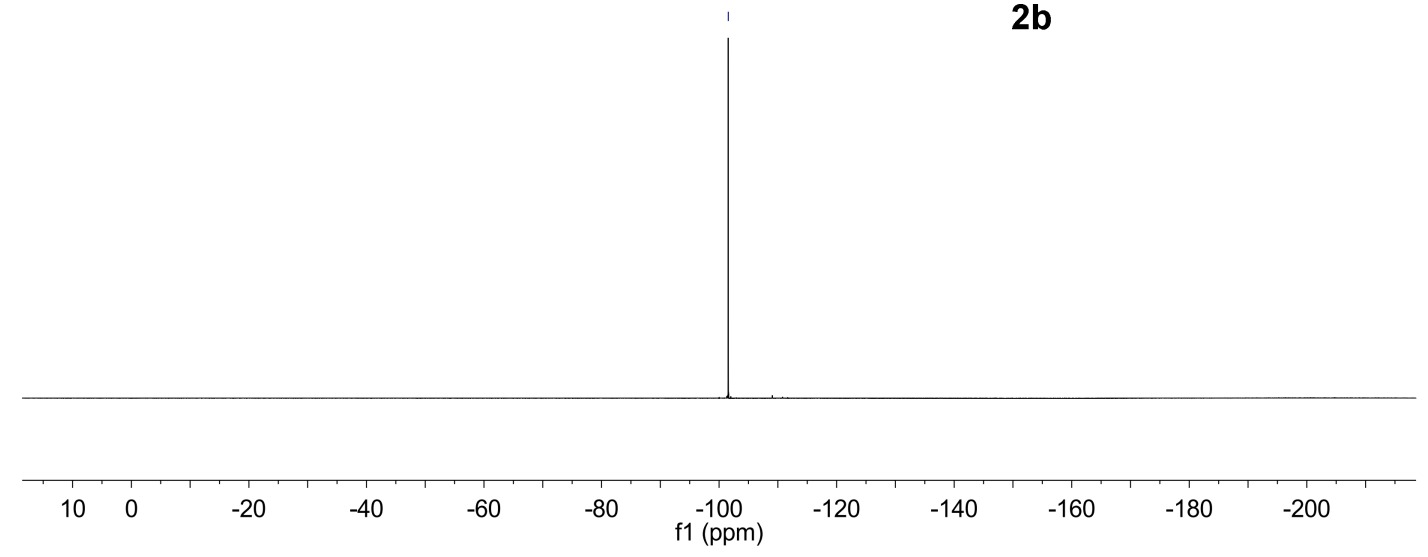

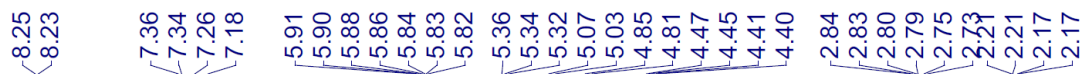
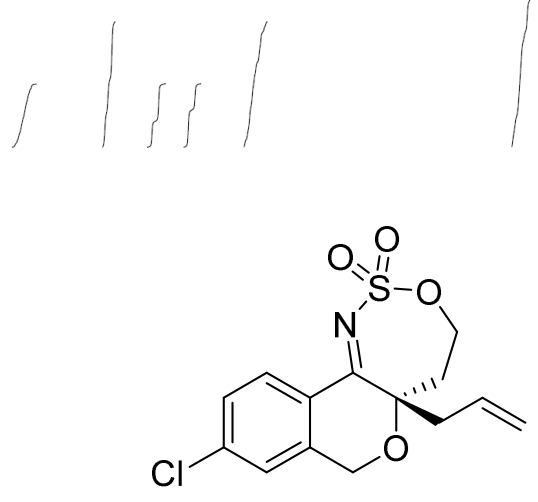

2c

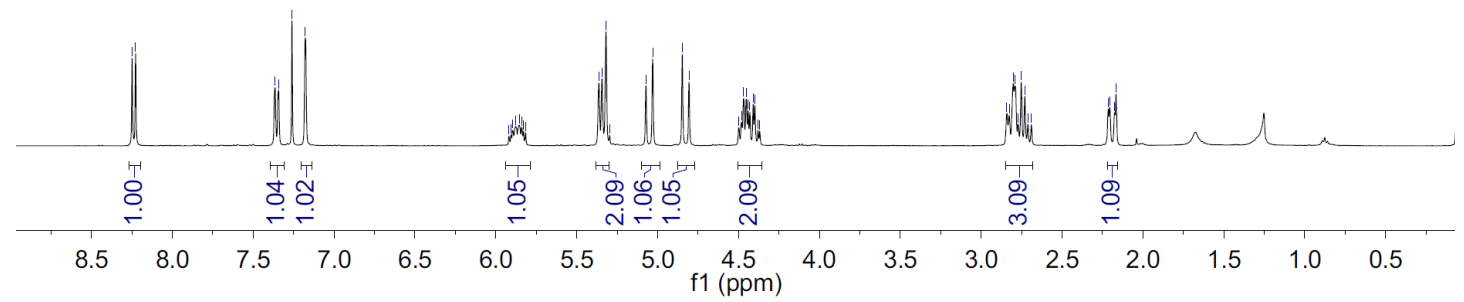



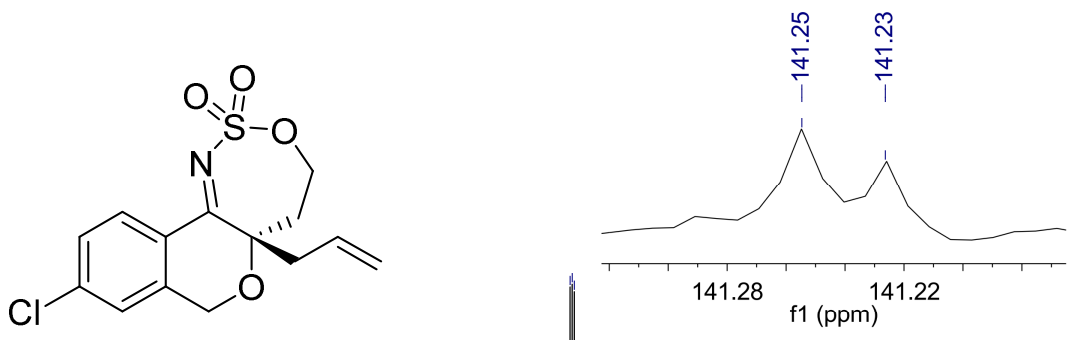

2c

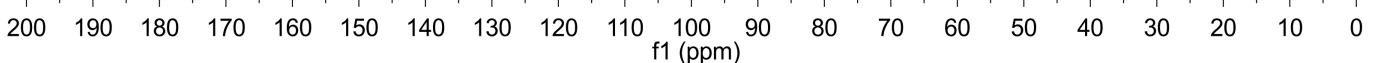

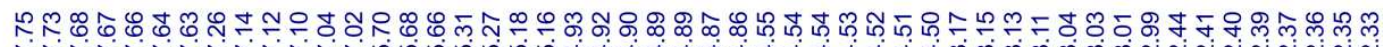

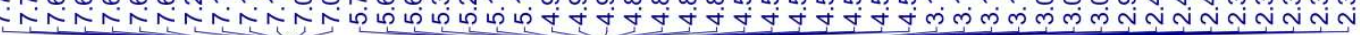<smiles>C=CC=C</smiles><smiles>C=CC1CCCCC1CC</smiles><smiles>c1ccccc1</smiles>

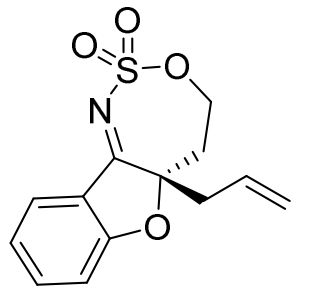

2d

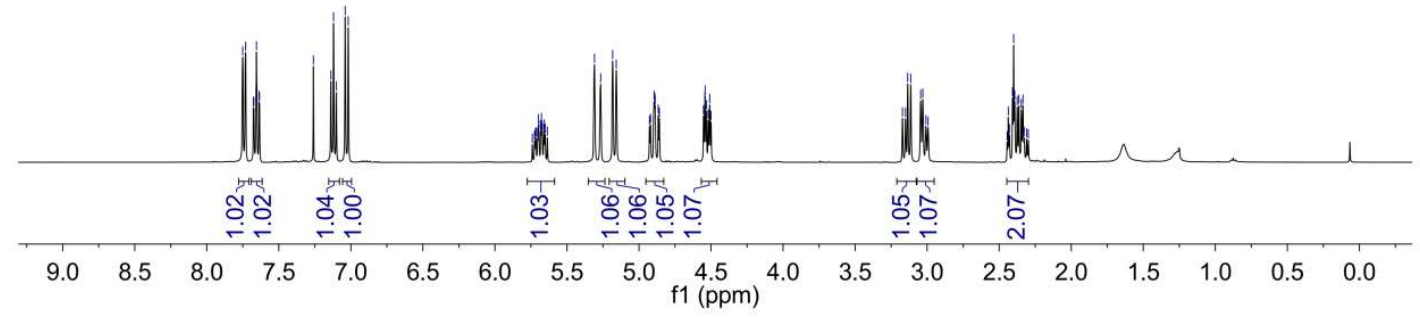




\begin{tabular}{|c|c|c|c|c|}
\hline $\begin{array}{l}0 \\
\text { o } \\
\stackrel{1}{1}\end{array}$ & 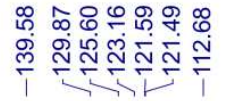 & 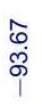 & 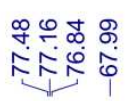 & 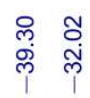 \\
\hline
\end{tabular}

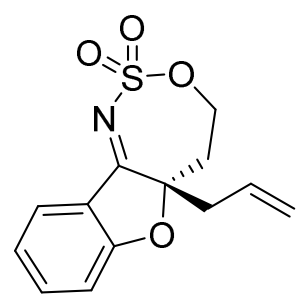

2d

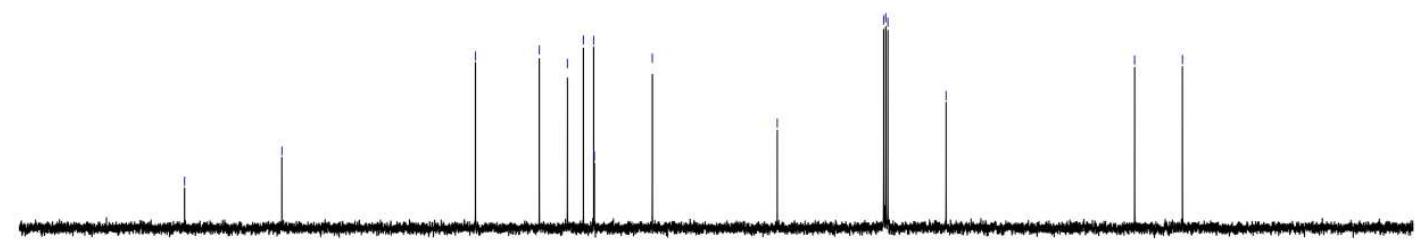

$\begin{array}{lllllllllllllllllllll}200 & 190 & 180 & 170 & 160 & 150 & 140 & 130 & 120 & \begin{array}{c}110 \\ \mathrm{f} 1(\mathrm{ppm})\end{array} & 90 & 80 & 70 & 60 & 50 & 40 & 30 & 20 & 10 & 0\end{array}$

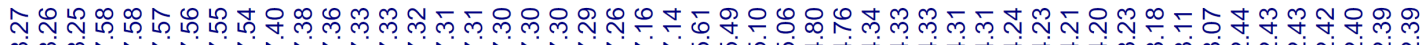
o do

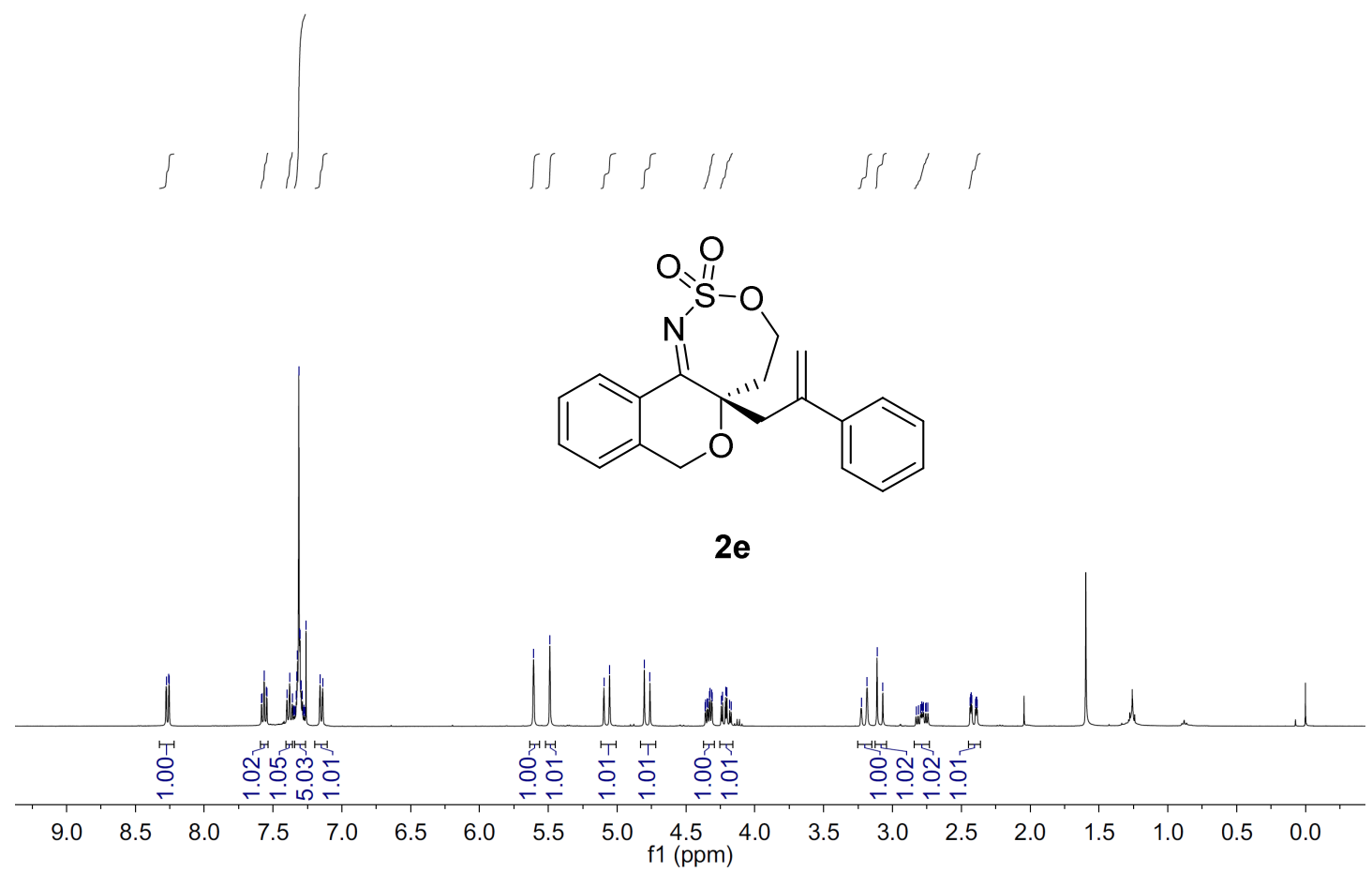


<smiles>C=C(C[C@@]12CCOS(=O)(=O)N=C1c1ccccc1CO2)c1ccccc1</smiles>

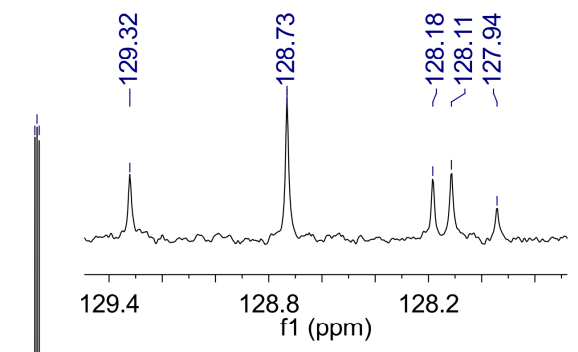

$2 e$

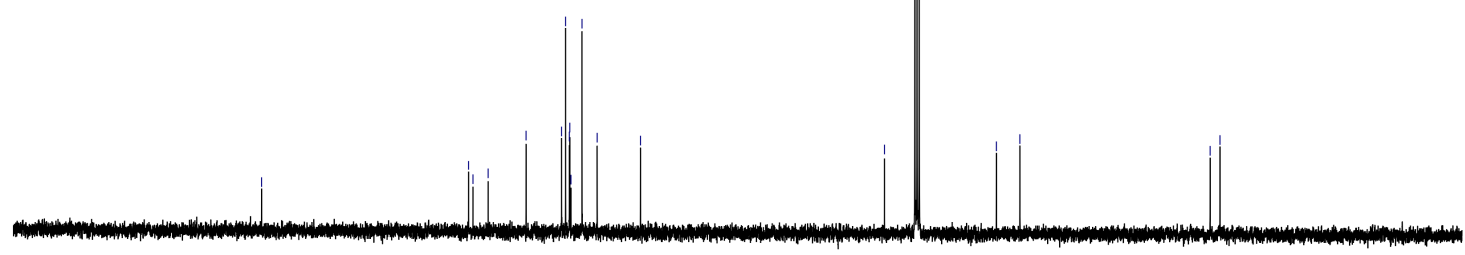

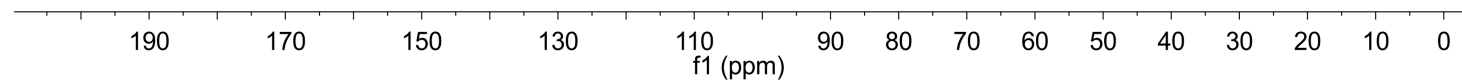

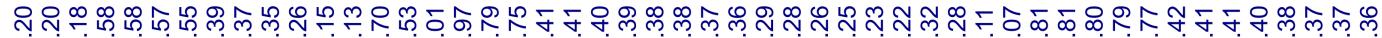

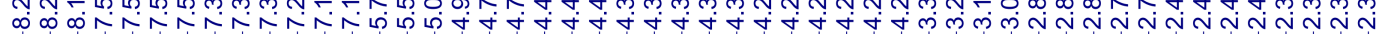
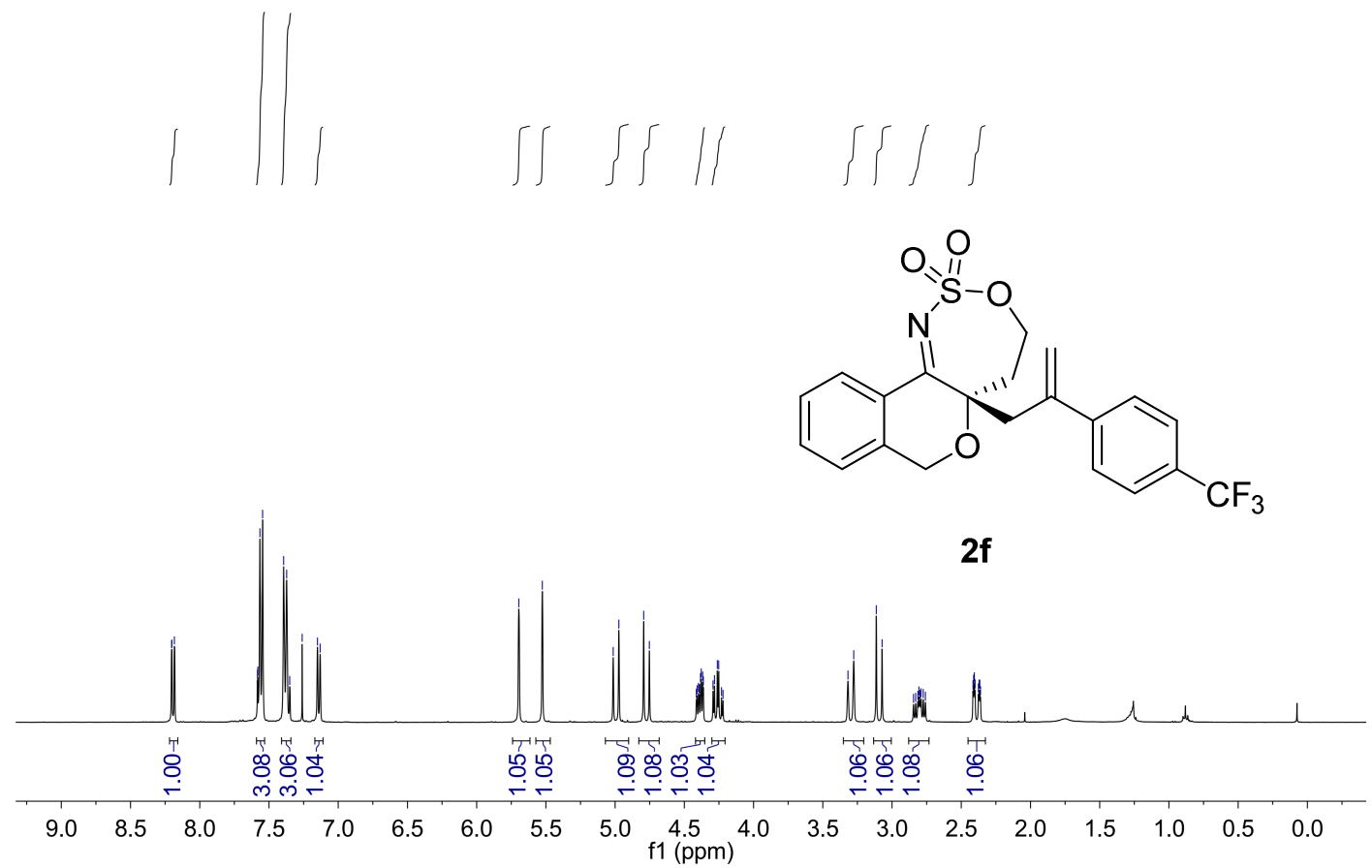

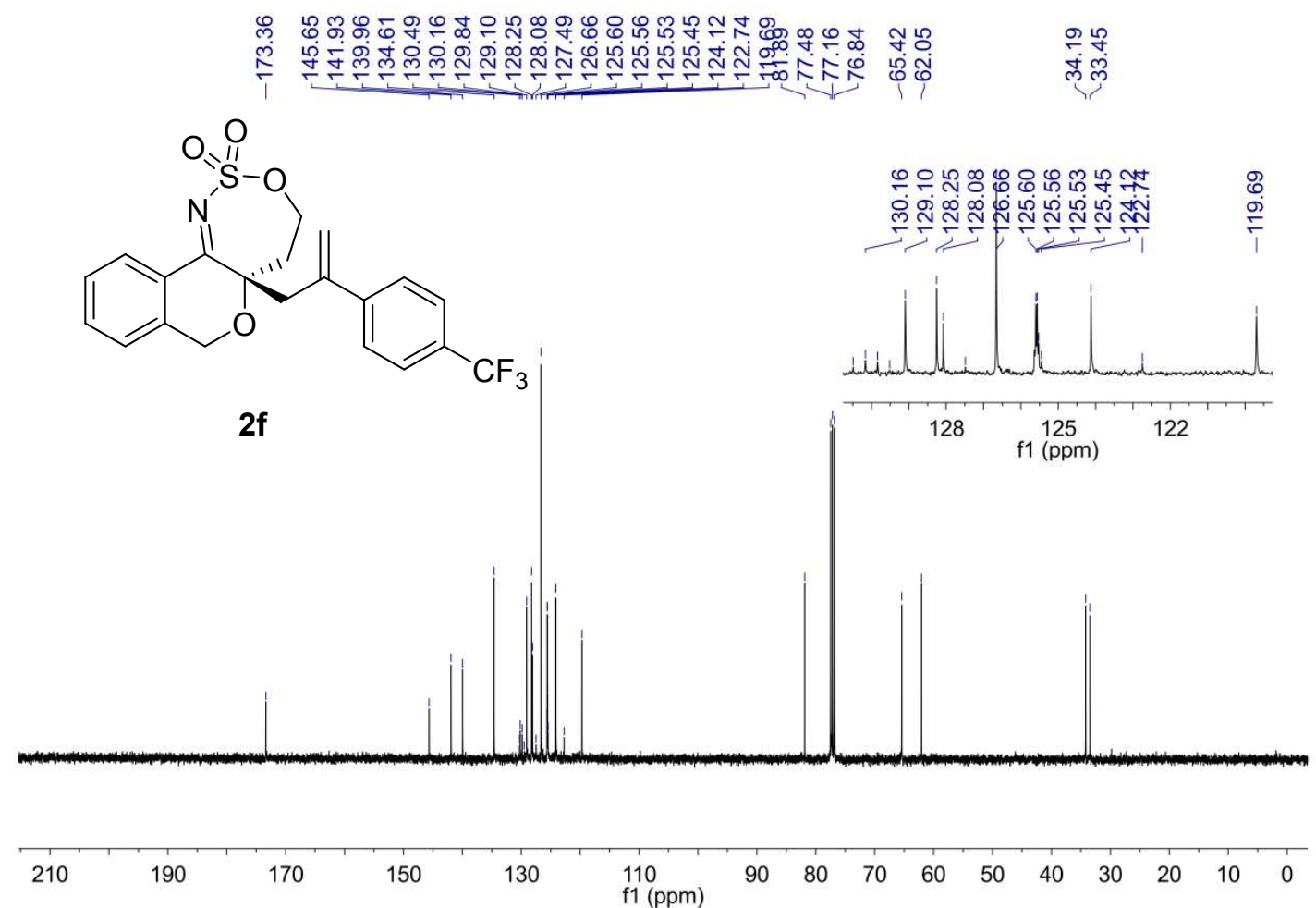

กับ

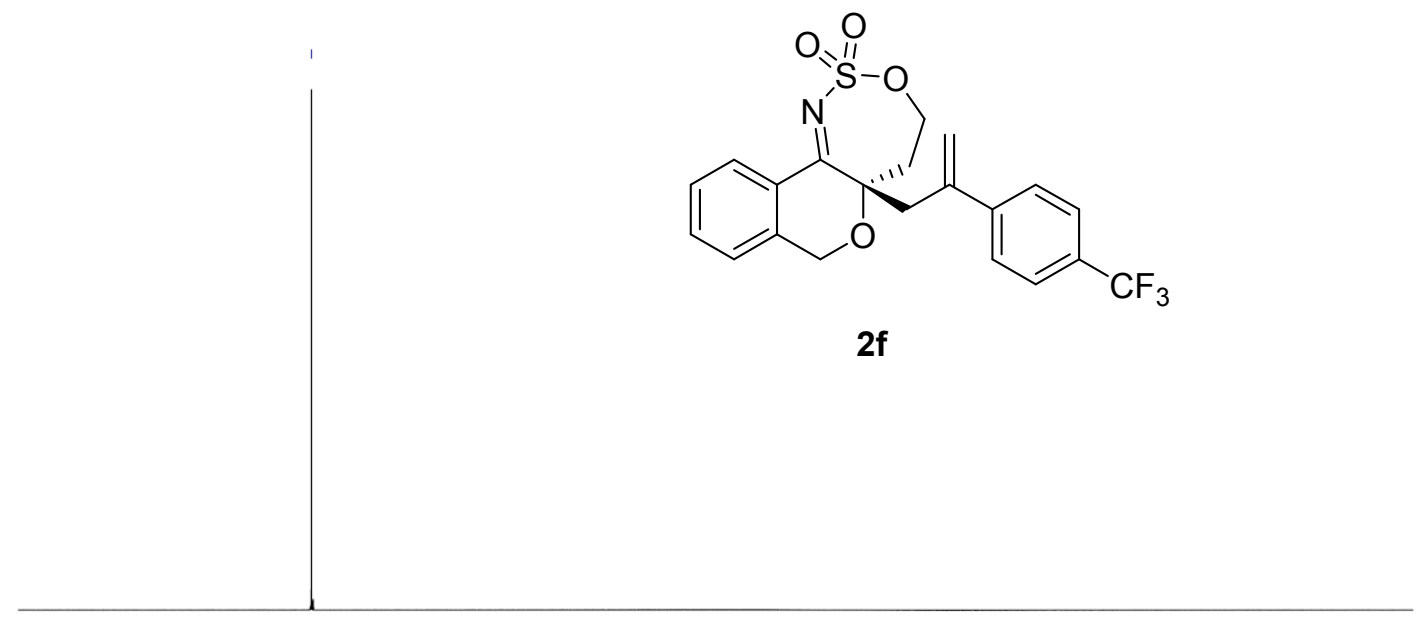

\begin{tabular}{llllllllllll}
\hline & -20 & -40 & -60 & -80 & -100 & -130 & -160 & -190 & -220 & -250 & -280
\end{tabular}


ఇైَ⿻

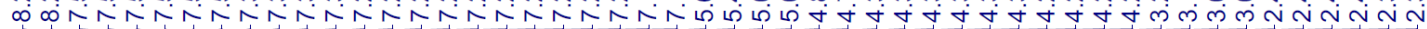

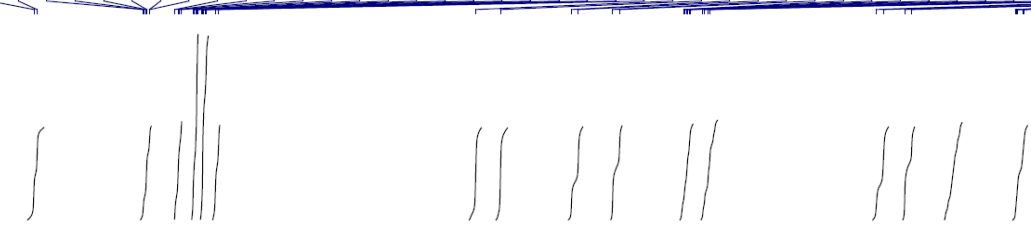<smiles>C=C1COS(=O)(=O)/N=C2/c3ccccc3CO[C@@]2(CC(=O)c2ccc(Cl)cc2)C1</smiles>

$2 g$

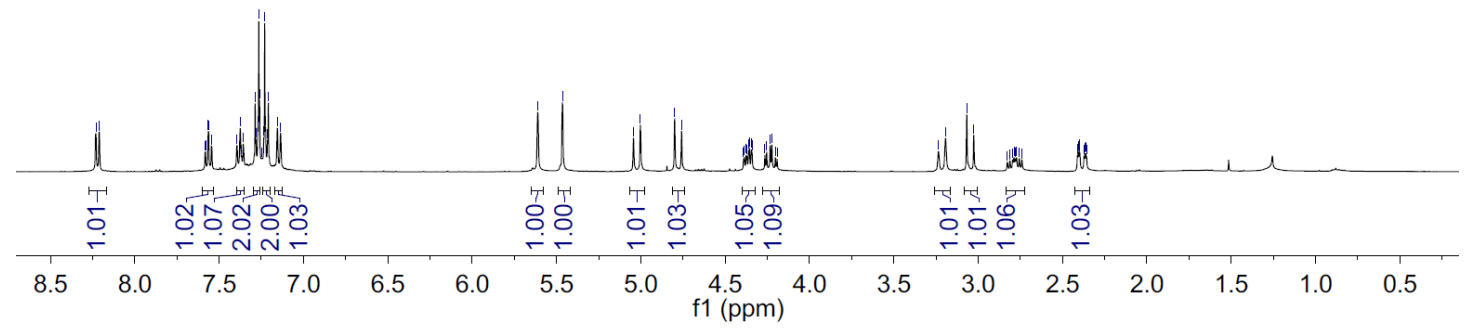

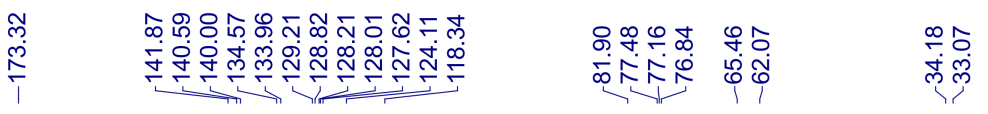<smiles>C=C1C[C@]2(CC(=C)c3ccc(Cl)cc3)OCc3ccccc3C2=NS(=O)(=O)O1</smiles>

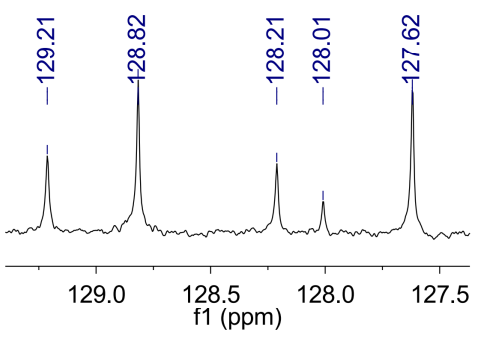

$2 g$

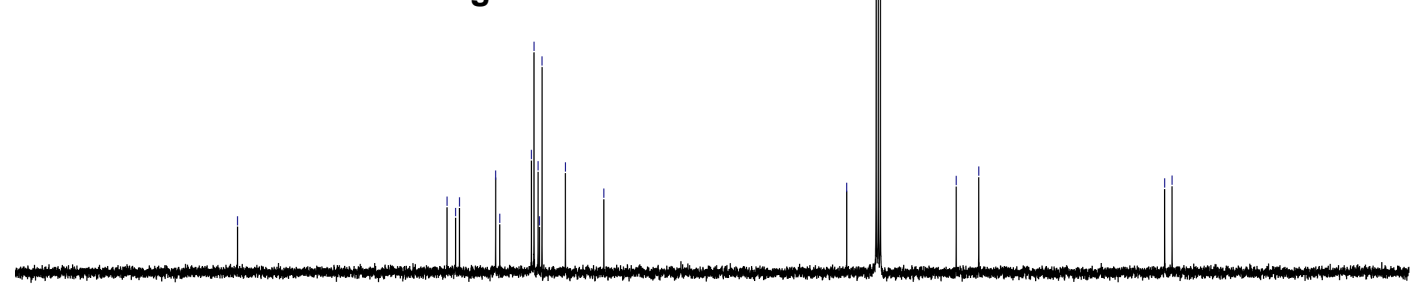

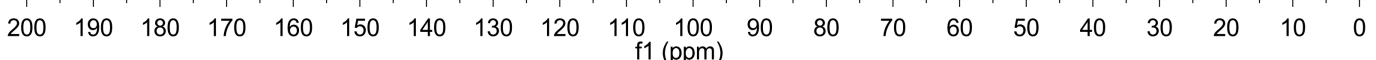




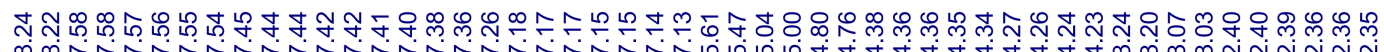

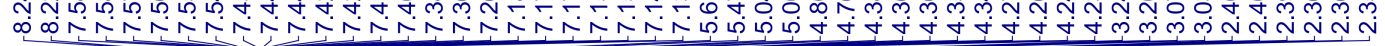

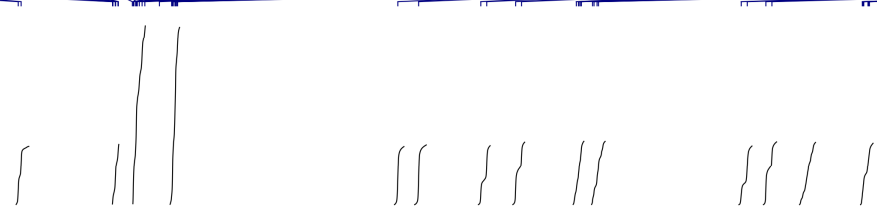<smiles>C=C(C[C@]12CCOS(=O)(=O)N=C1c1ccccc1CO2)c1ccc(Br)cc1</smiles>

$2 \mathrm{~h}$

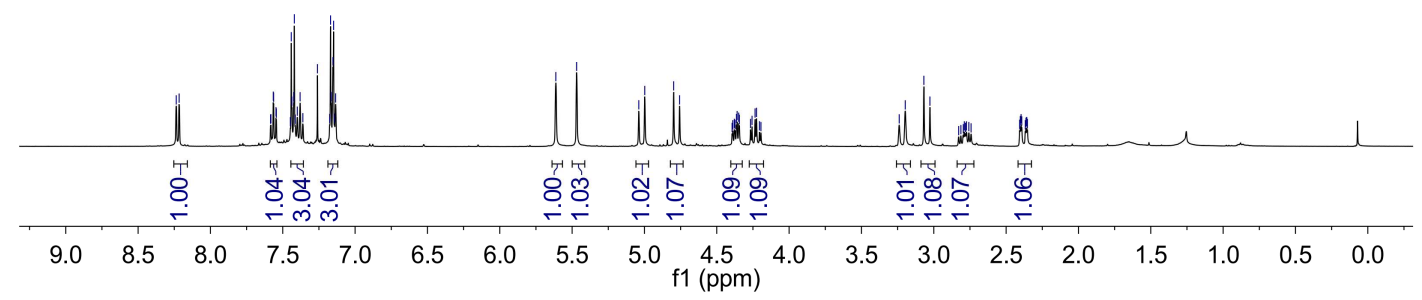

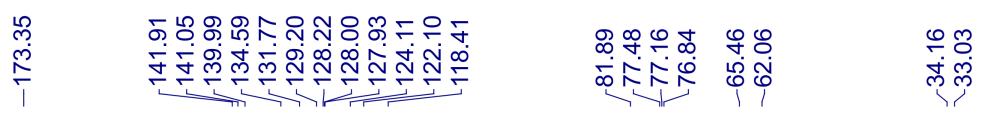<smiles>C=C(C[C@@]12CCOS(=O)(=O)N=C1c1ccccc1CO2)c1ccc(Br)cc1</smiles>

$2 \mathrm{~h}$

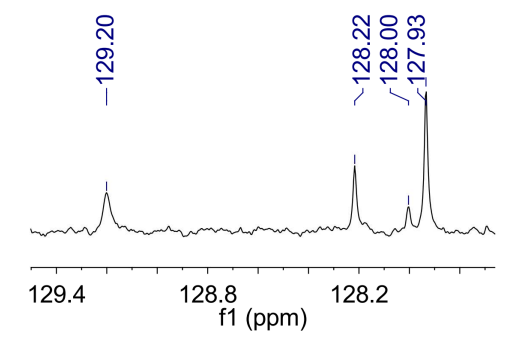

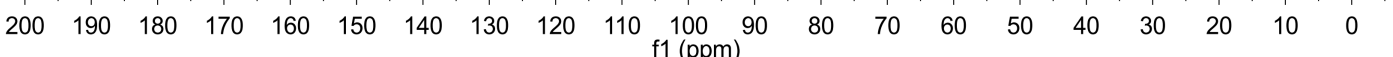




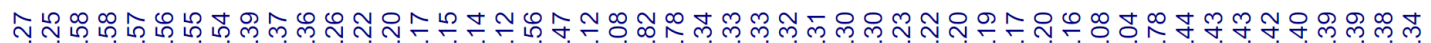

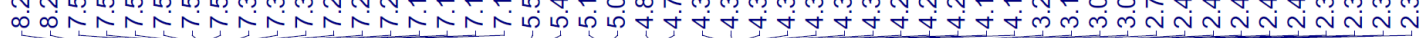
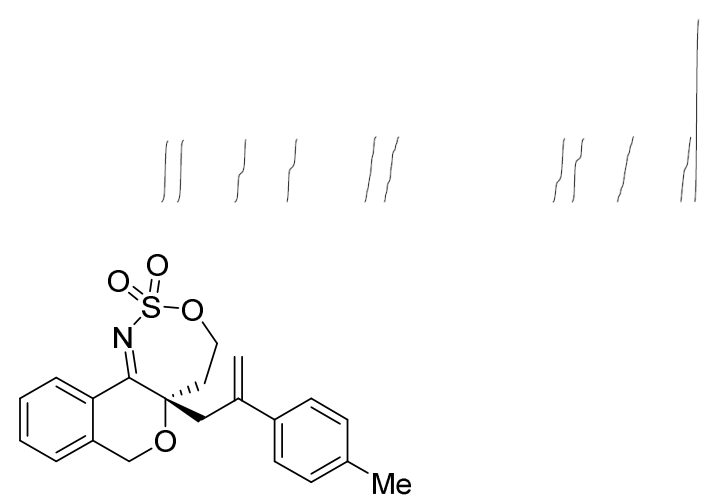

2i

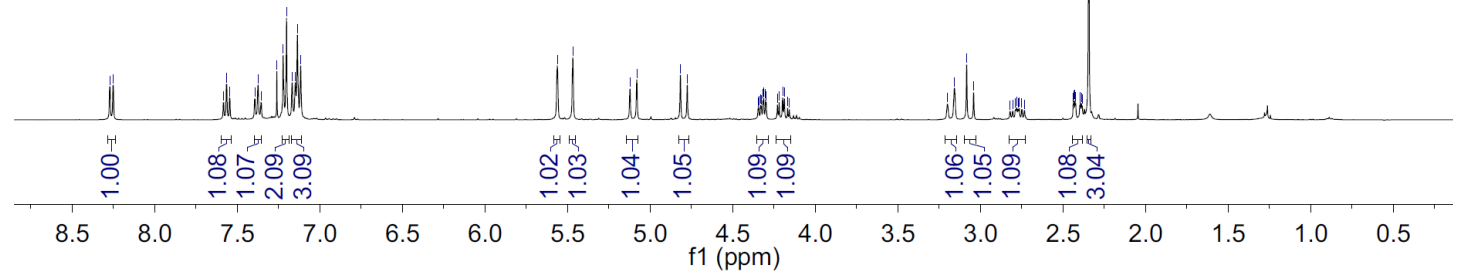

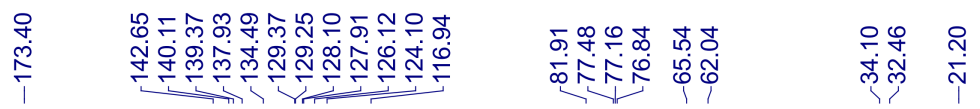<smiles>C=C(C[C@@]12CCOS(=O)(=O)N=C1c1ccccc1CO2)c1ccc(C)cc1</smiles>

2i

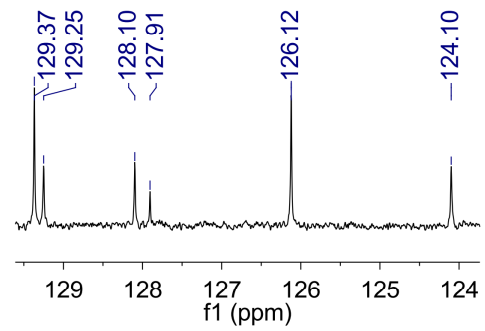

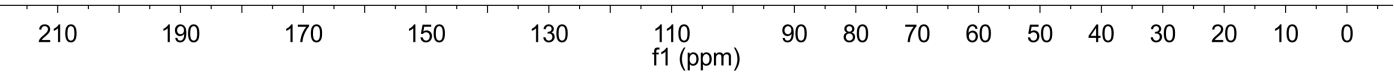




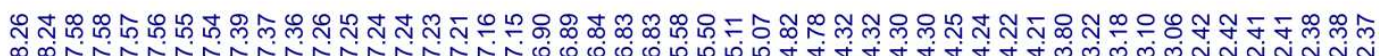

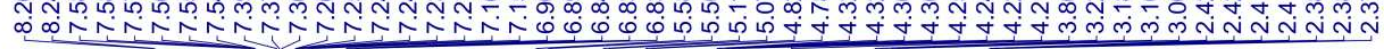

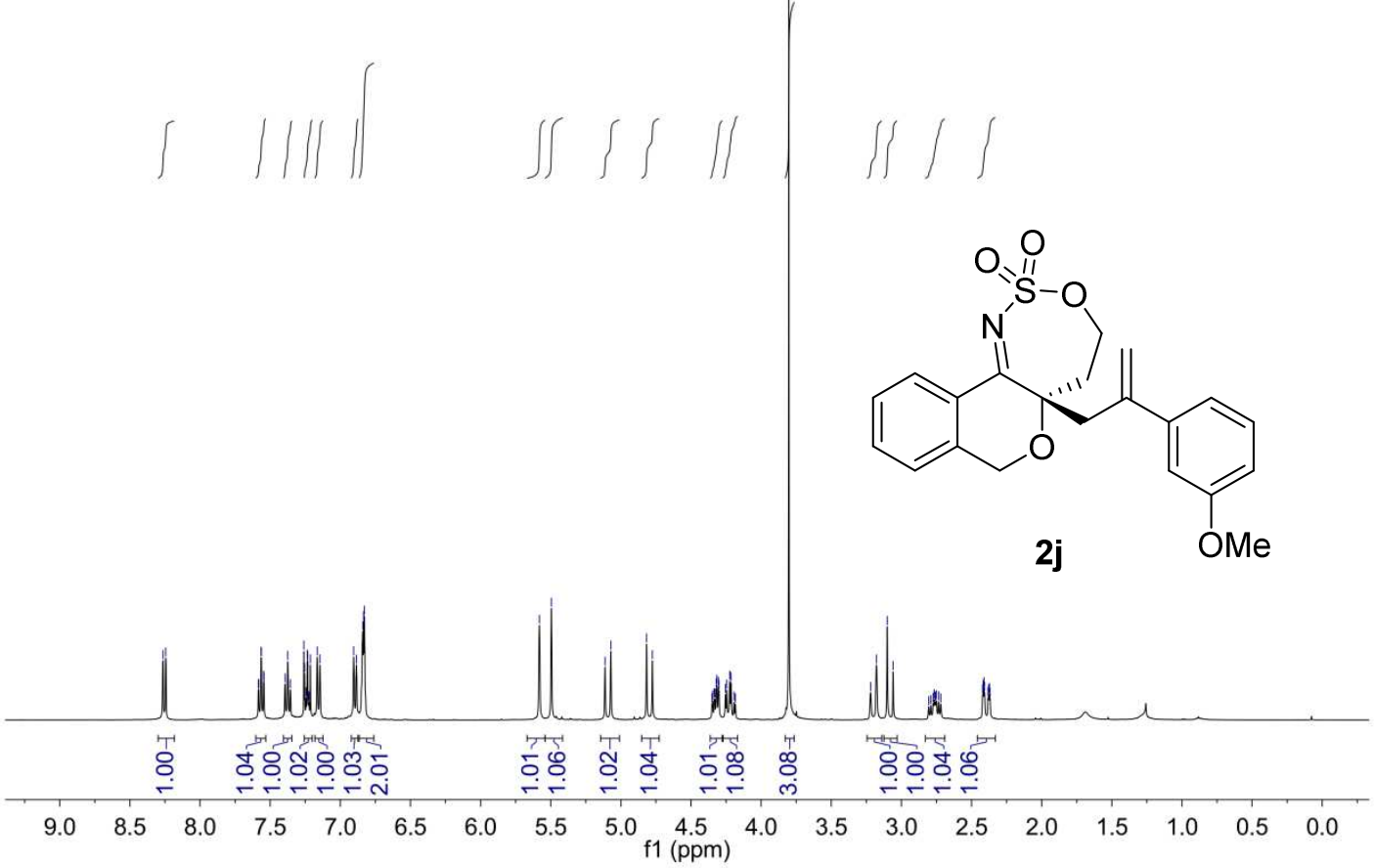

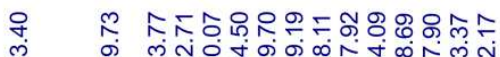

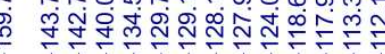

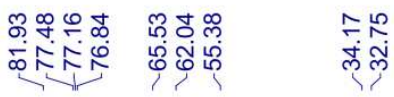

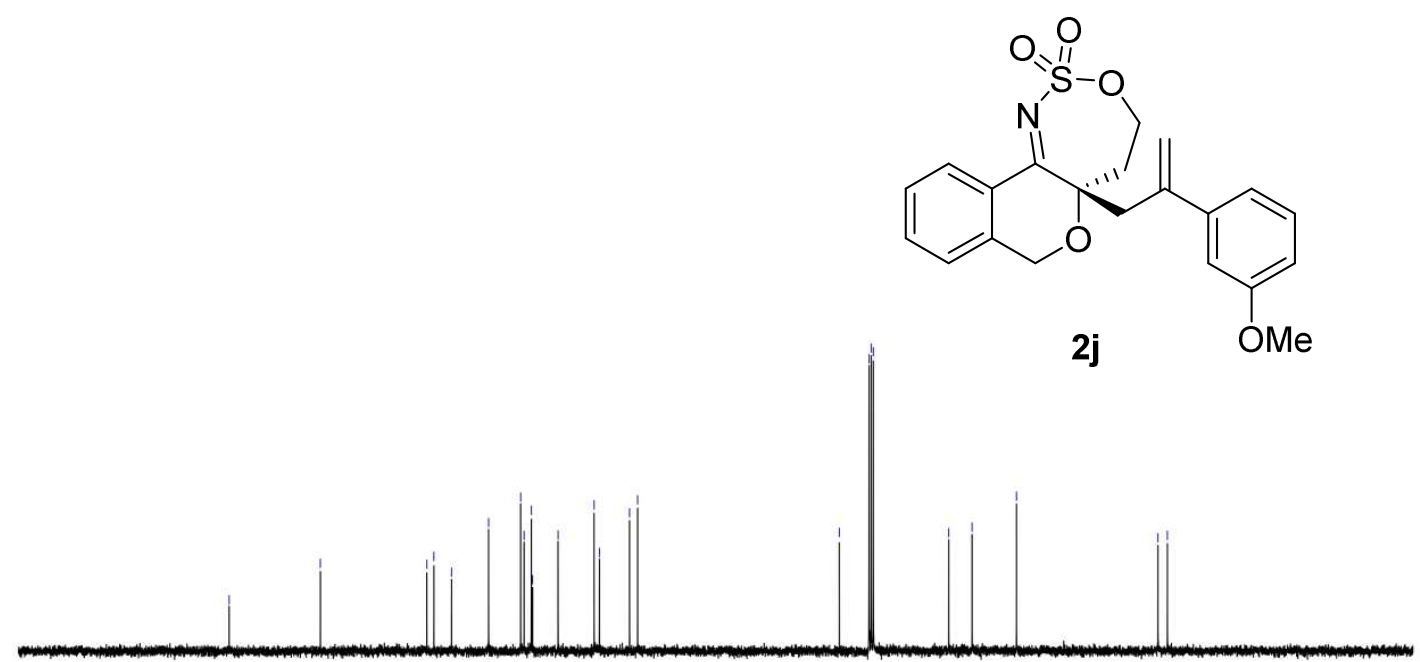

$\begin{array}{llllllllllllllllllll}200 & 190 & 180 & 170 & 160 & 150 & 140 & 130 & 120 & 110 \begin{array}{l}100 \\ \mathrm{f} 1(\mathrm{ppm})\end{array} & 90 & 80 & 70 & 60 & 50 & 40 & 30 & 20 & 10 & 0\end{array}$ 


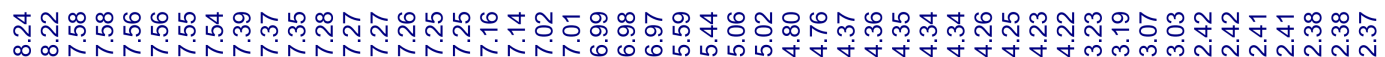

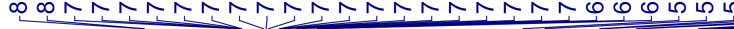
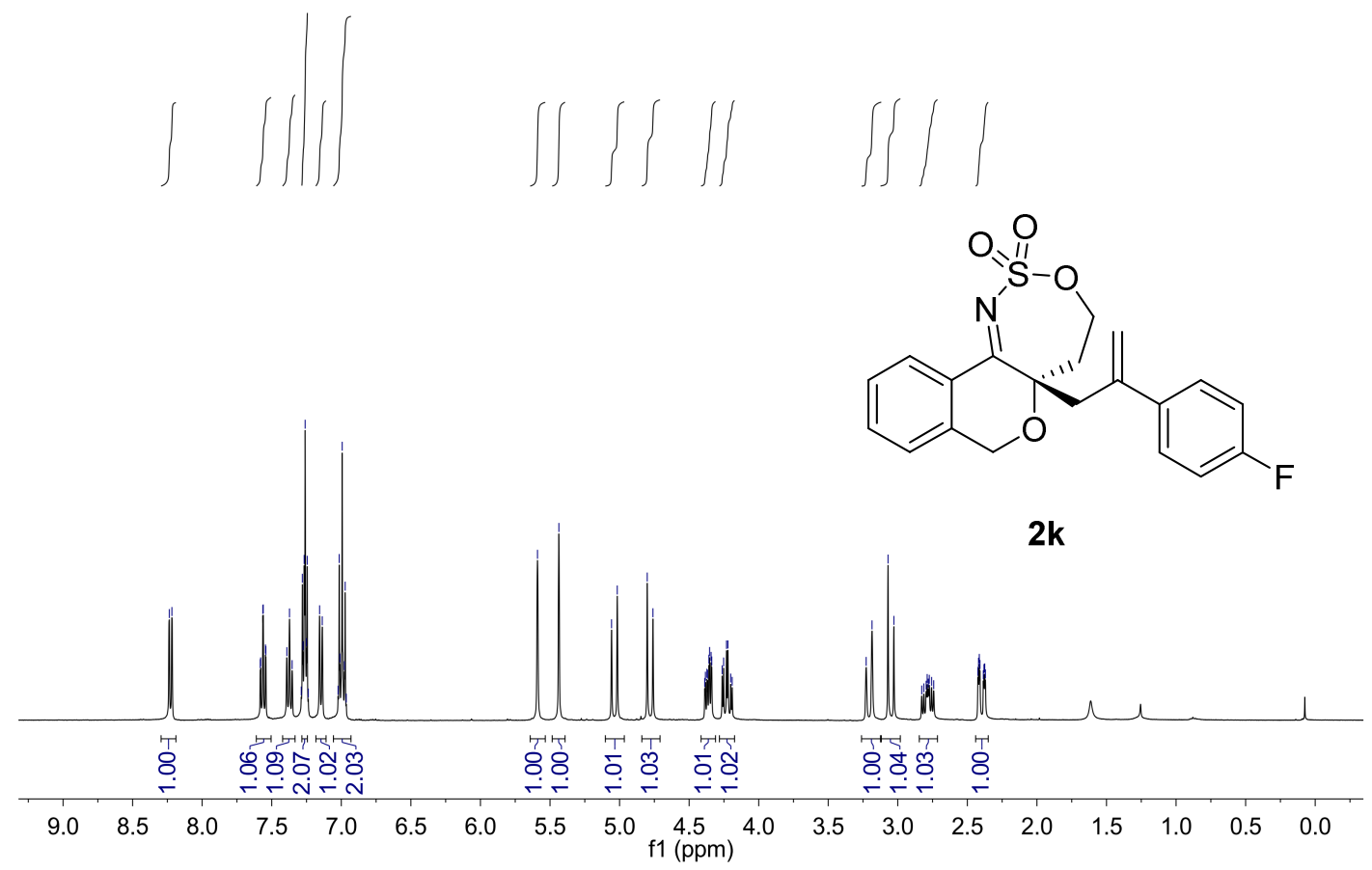

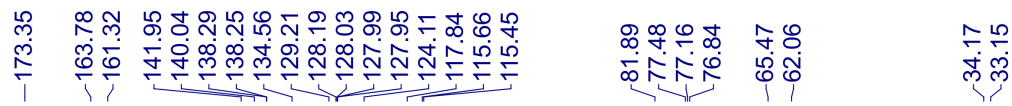
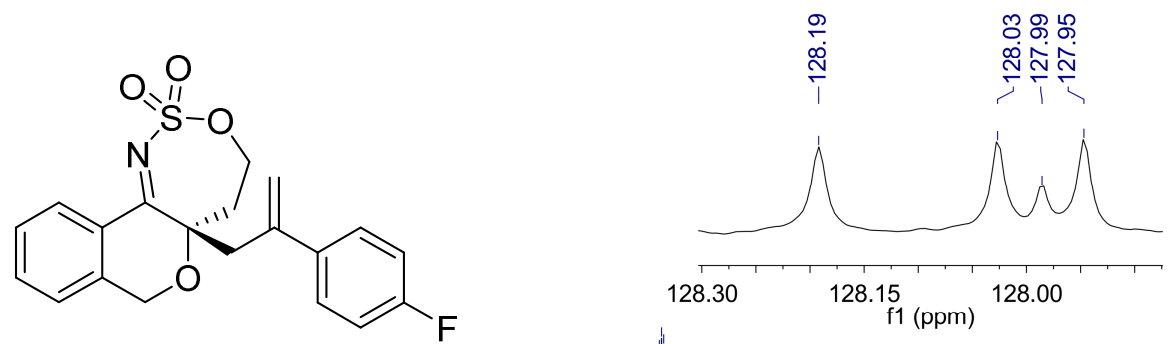

2k

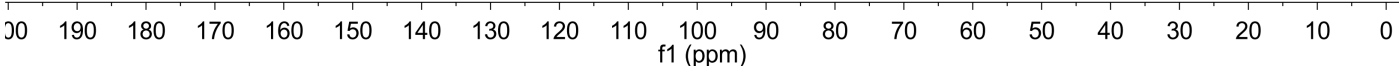


<smiles>C=C(C[C@]12CCOS(=O)(=O)N=C1c1ccccc1CO2)c1ccc(F)cc1</smiles>

2k

\begin{tabular}{|c|c|c|c|c|c|c|c|c|c|c|c|}
\hline 10 & 0 & -20 & -40 & -60 & -80 & $\begin{array}{c}-100 \\
\mathrm{f} 1(\mathrm{ppm})\end{array}$ & -120 & -140 & -160 & -180 & -200 \\
\hline
\end{tabular}

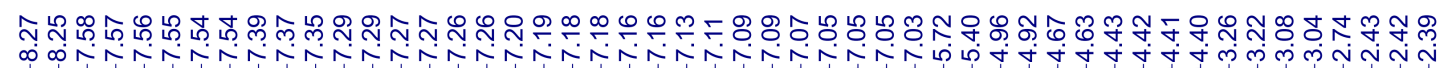
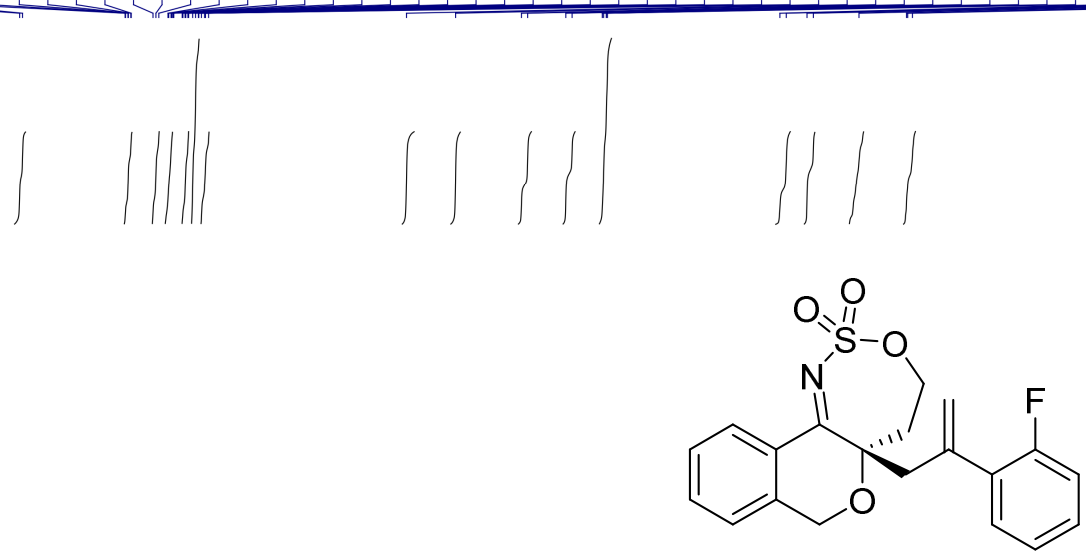

2I

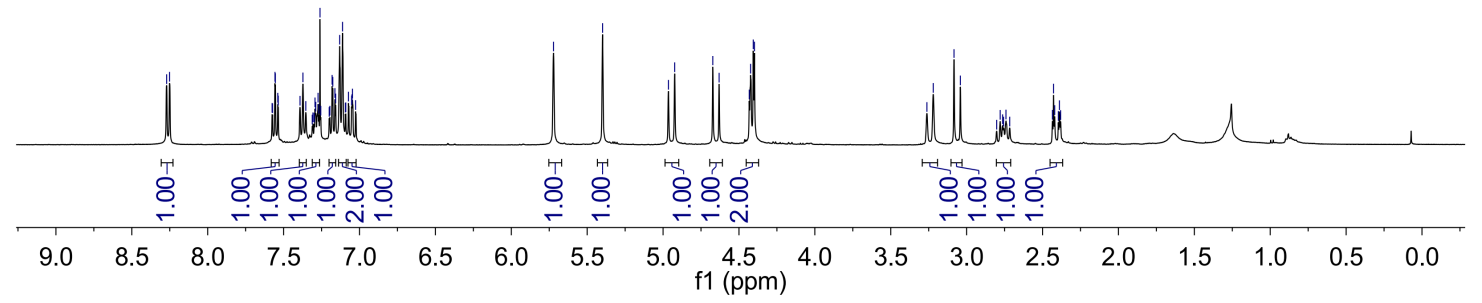




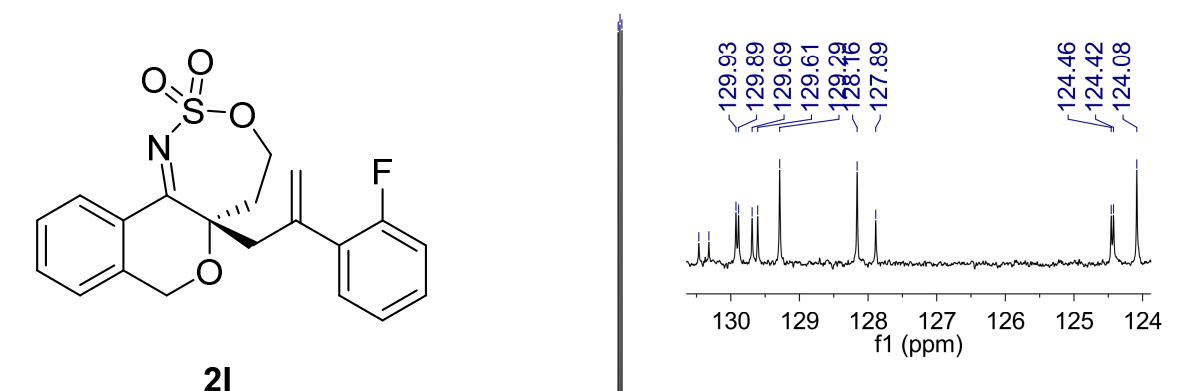

2I

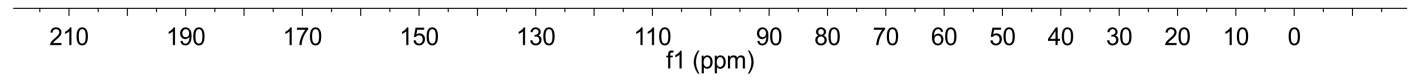

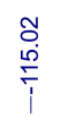

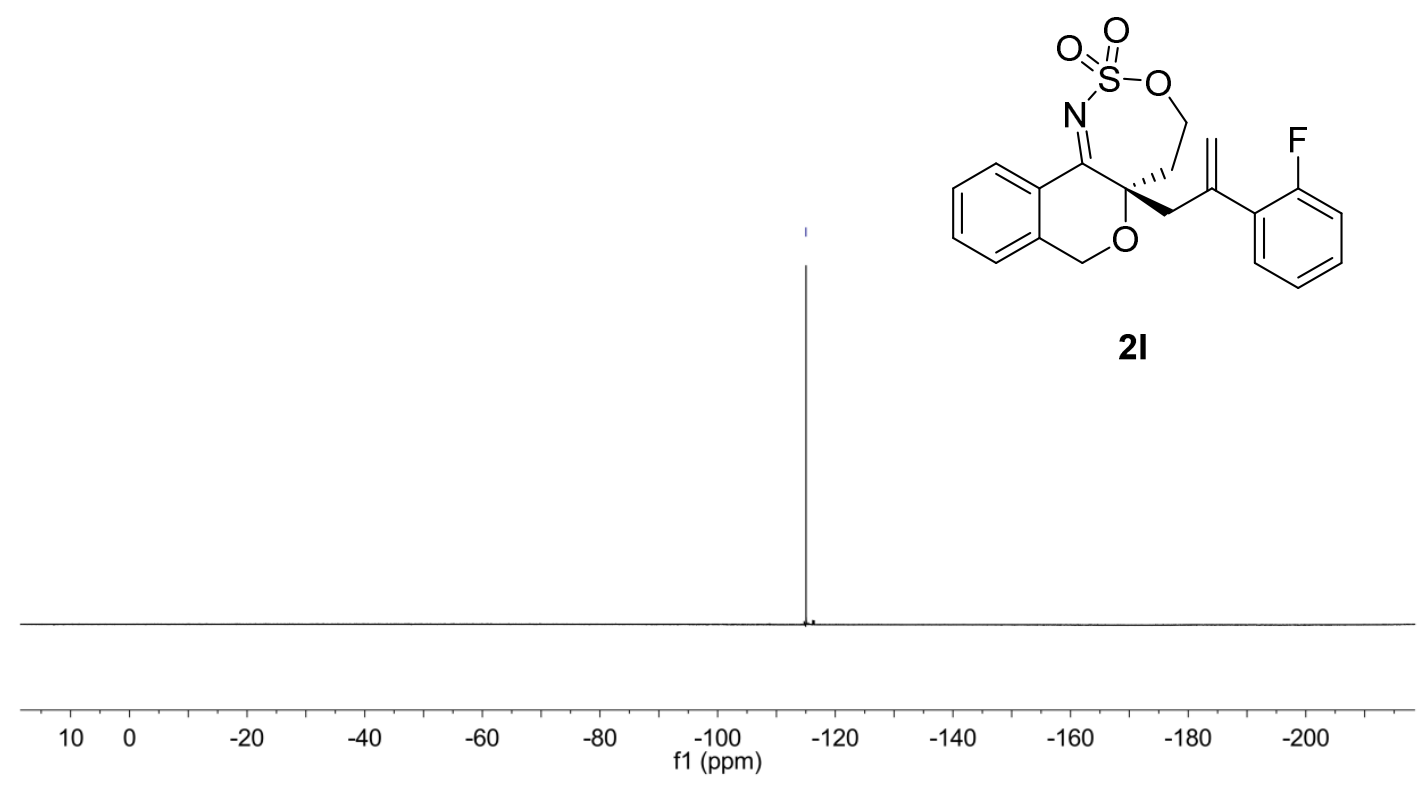




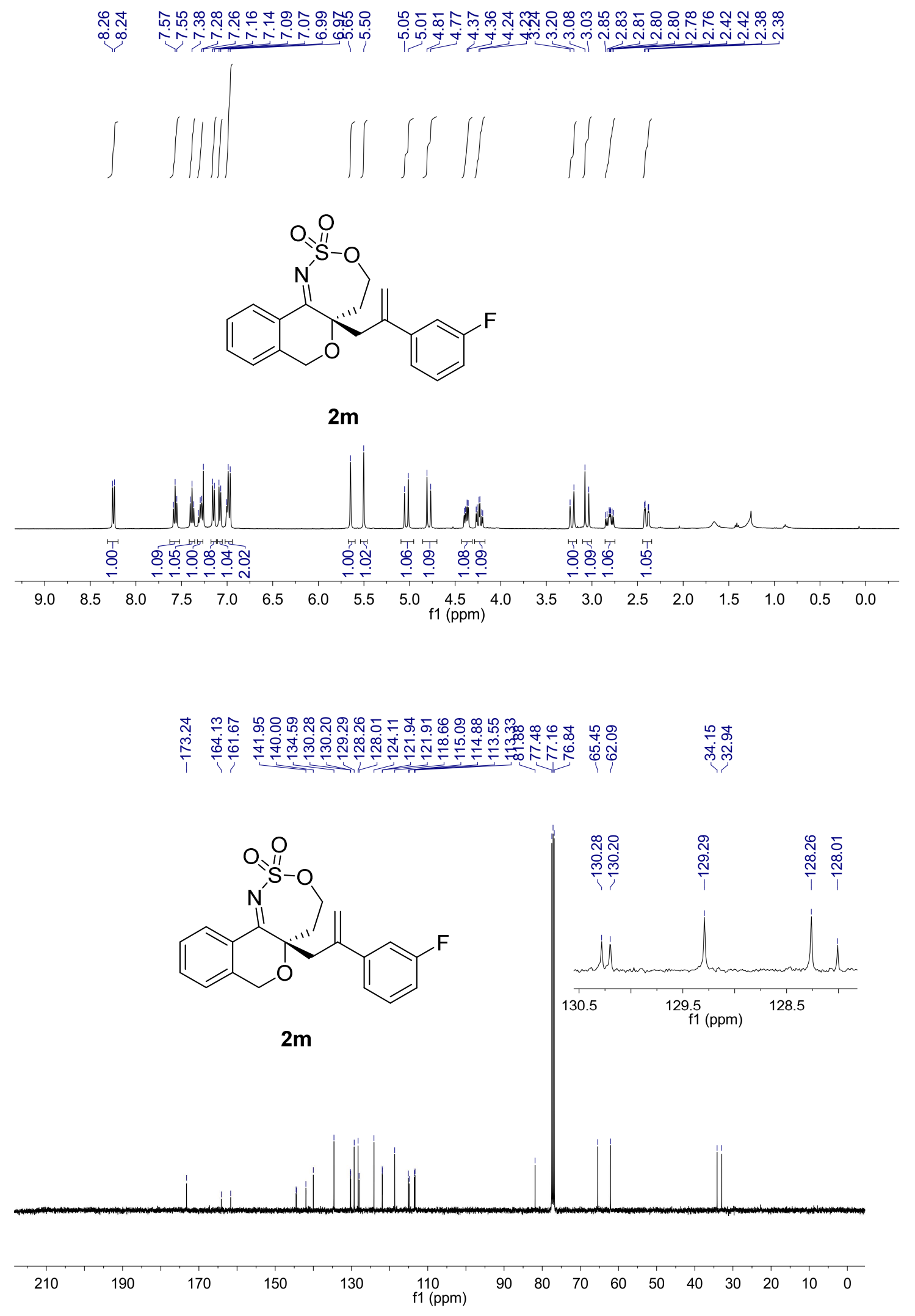




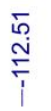

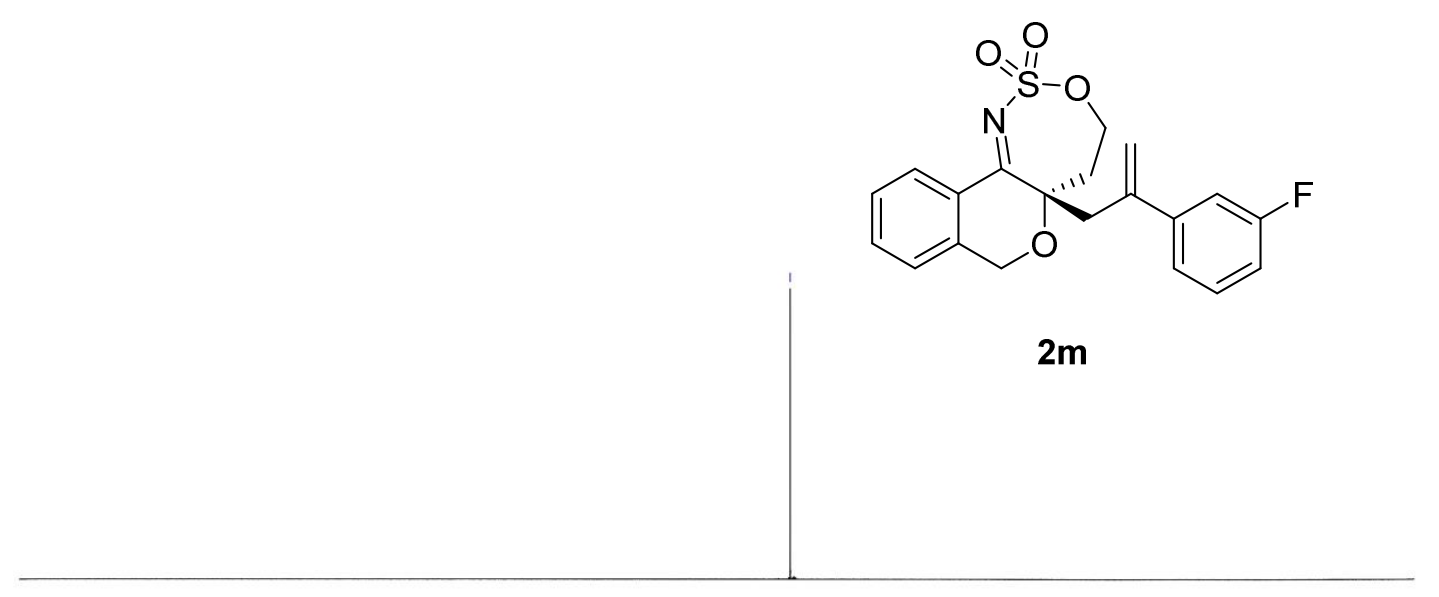

\begin{tabular}{|c|c|c|c|c|c|c|c|c|c|c|c|}
\hline 10 & 0 & -20 & -40 & -60 & -80 & $\begin{array}{c}-100 \\
\mathrm{f} 1(\mathrm{ppm})\end{array}$ & -120 & -140 & -160 & -180 & -200 \\
\hline
\end{tabular}

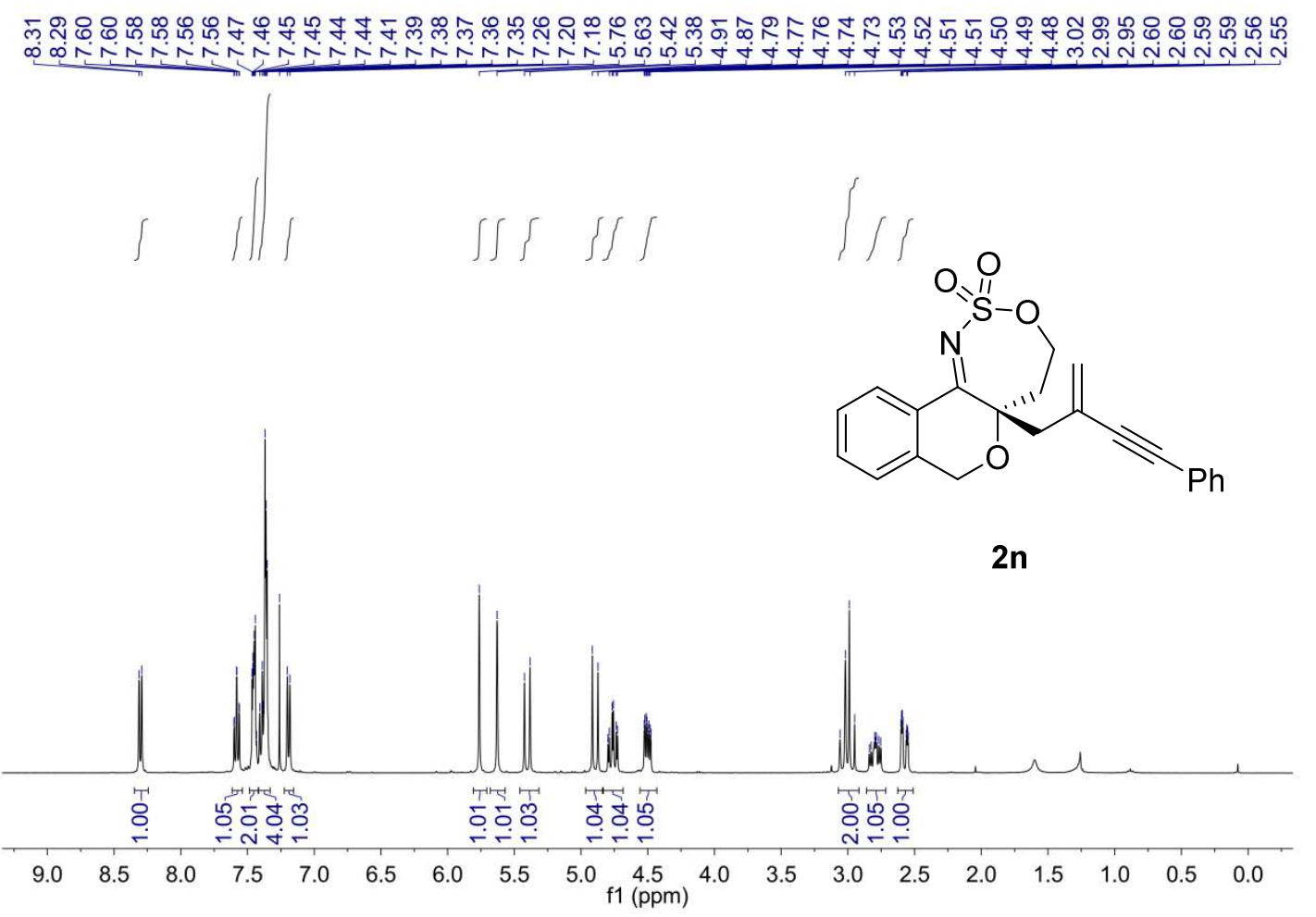


<smiles>C=C(C#CPc1ccccc1)C[C@]12CCOS(=O)(=O)N=C1c1ccccc1CO2</smiles>

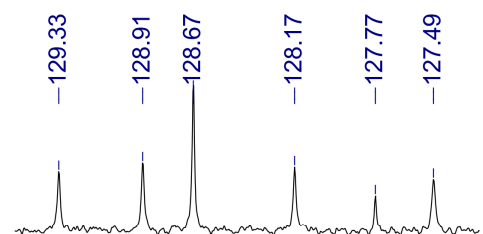

$2 n$

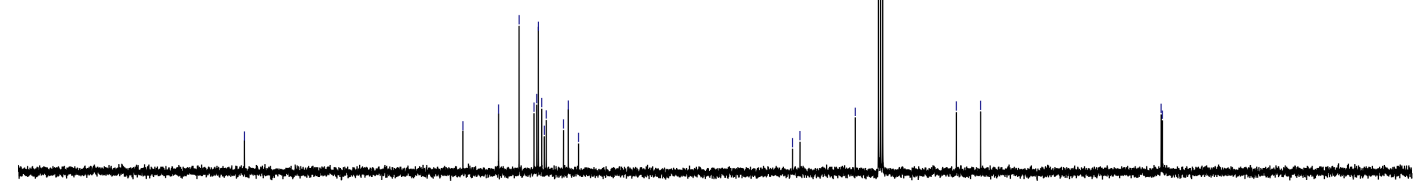

$\begin{array}{llllllllllllllllllllll}200 & 190 & 180 & 170 & 160 & 150 & 140 & 130 & 120 & 110 & 100 & 90 & 80 & 70 & 60 & 50 & 40 & 30 & 20 & 10 & 0\end{array}$

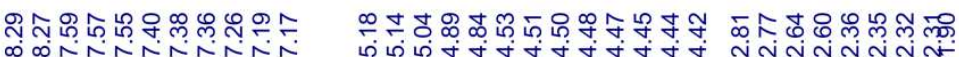

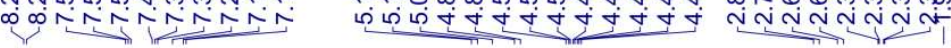

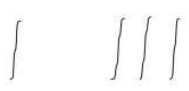

$\left.\int 1\right\}$

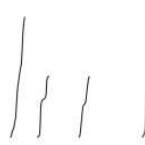<smiles>C=C(C)C[C@@]12CCOS(=O)(=O)N=C1c1ccccc1CO2</smiles>

20

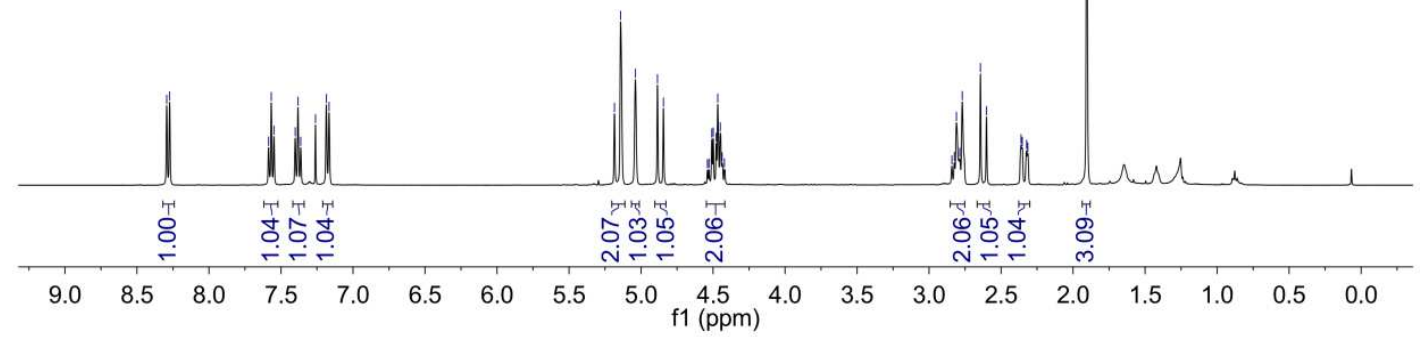


<smiles>C=C(C)C[C@@]12CCOS(=O)(=O)N=C1c1ccccc1CO2</smiles>

20

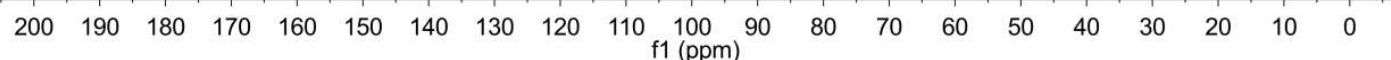

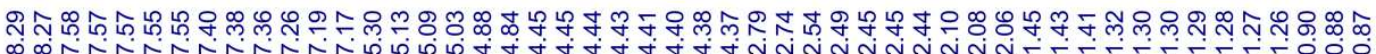

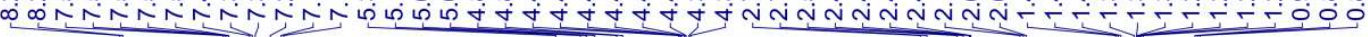<smiles>C=C(CCCCC)C[C@]12CCOS(=O)(=O)N=C1c1ccccc1CO2</smiles>

$2 p$

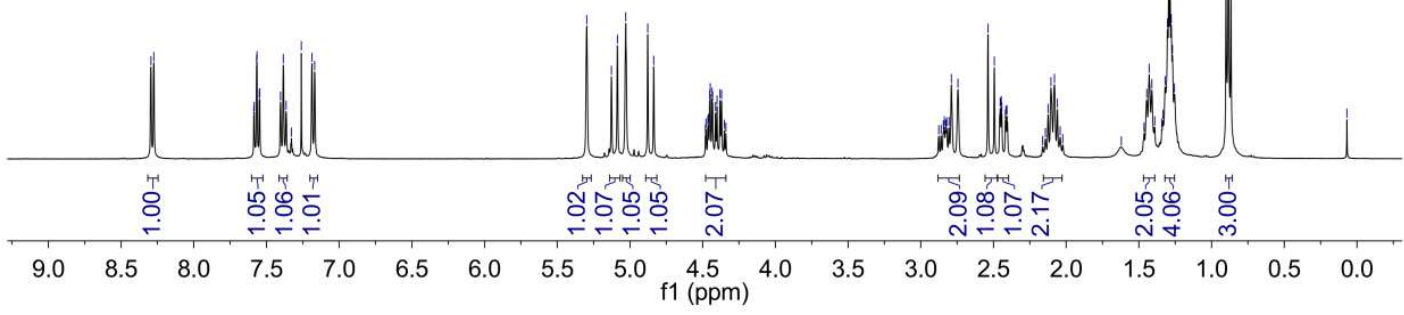




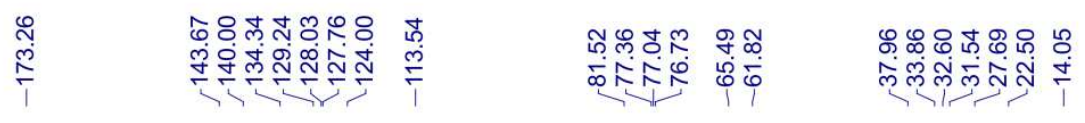<smiles>C=C(CCCCC)C[C@]12CCOS(=O)(=O)N=C1c1ccccc1CO2</smiles>

$2 p$

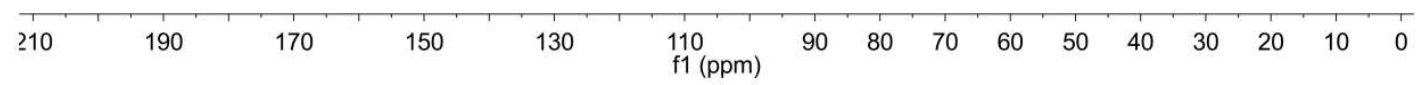

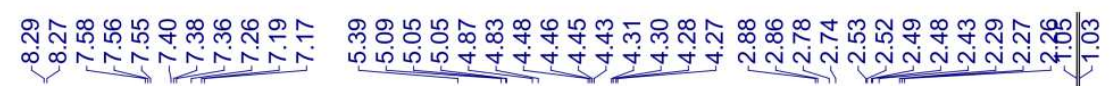<smiles></smiles>

$2 q$

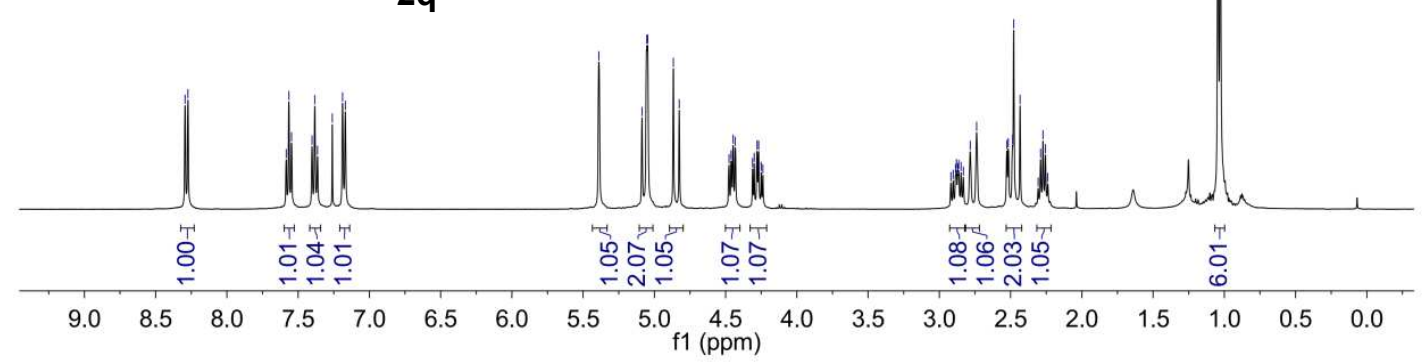


<smiles>C=C(C[C@@]12CCOS(=O)(=O)N=C1c1ccccc1CO2)C(C)C</smiles>

$2 q$

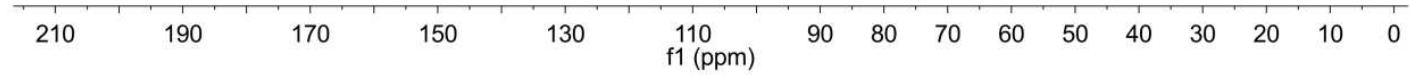

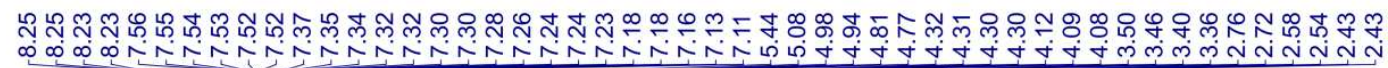

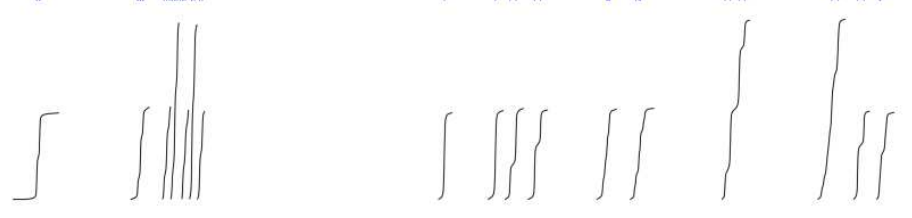<smiles>C=C(Cc1ccccc1)C[C@]12CCOS(=O)(=O)N=C1c1ccccc1CO2</smiles>

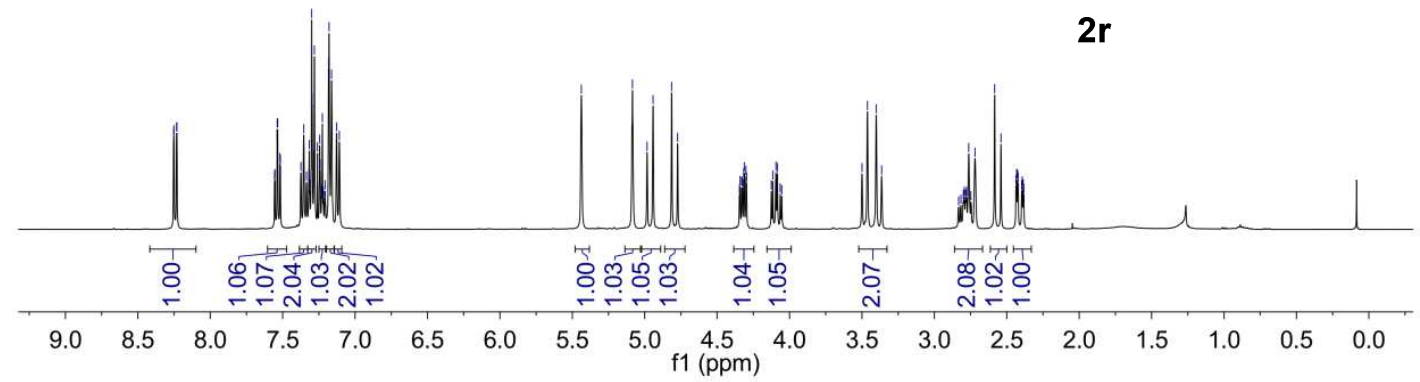



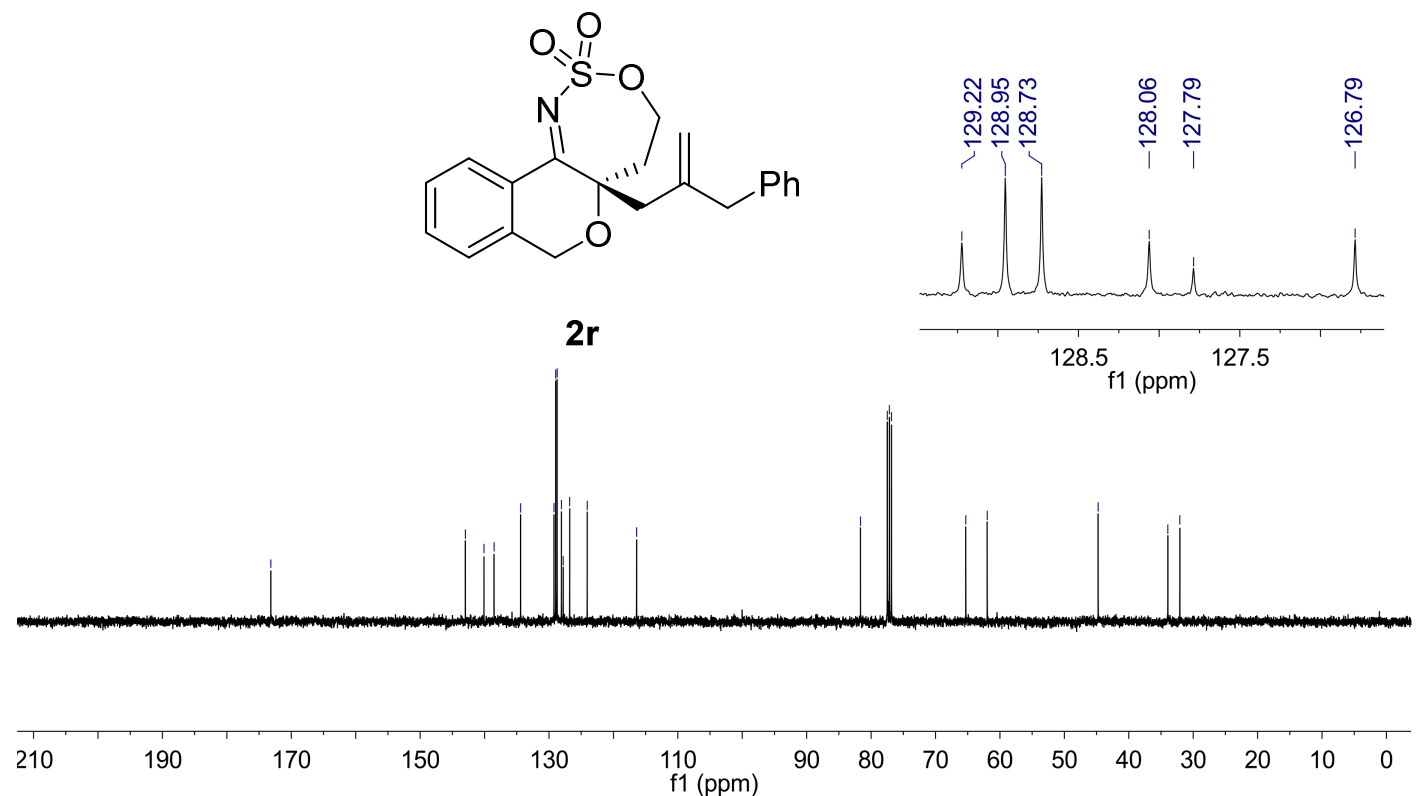

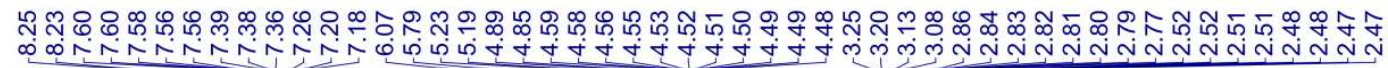
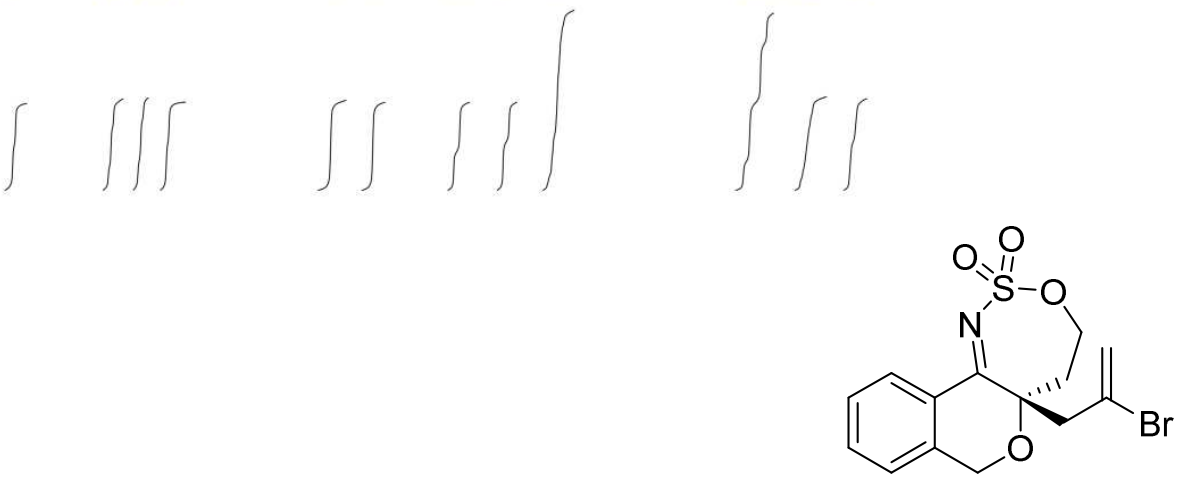

2s

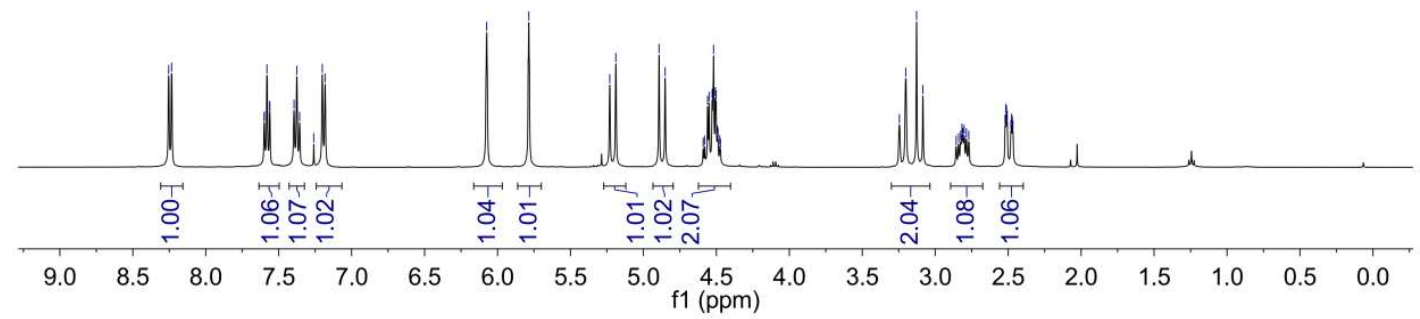



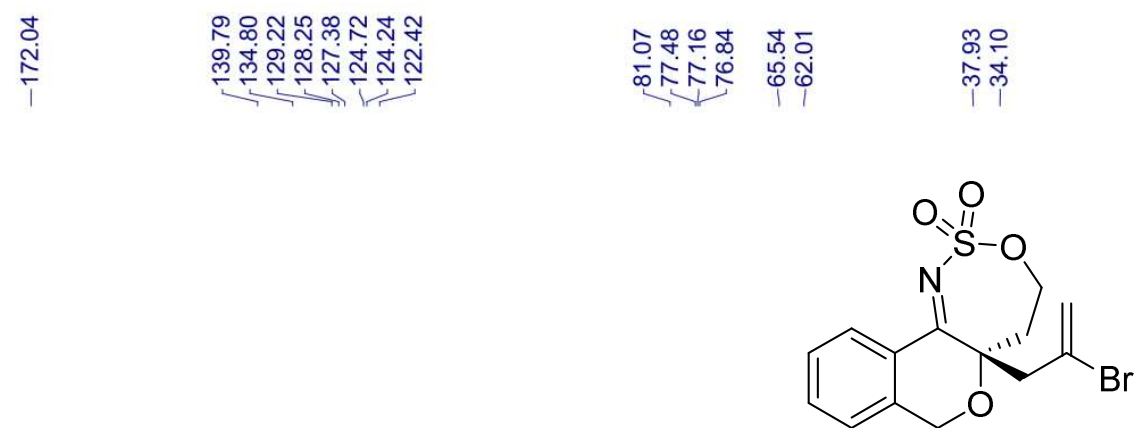

2s

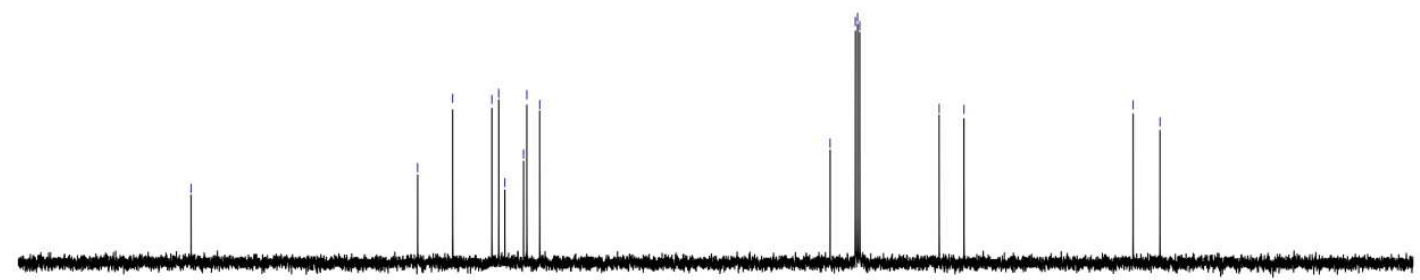

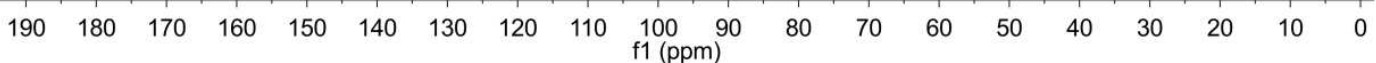

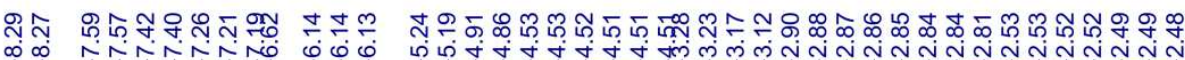

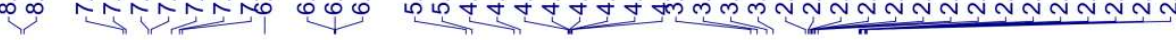
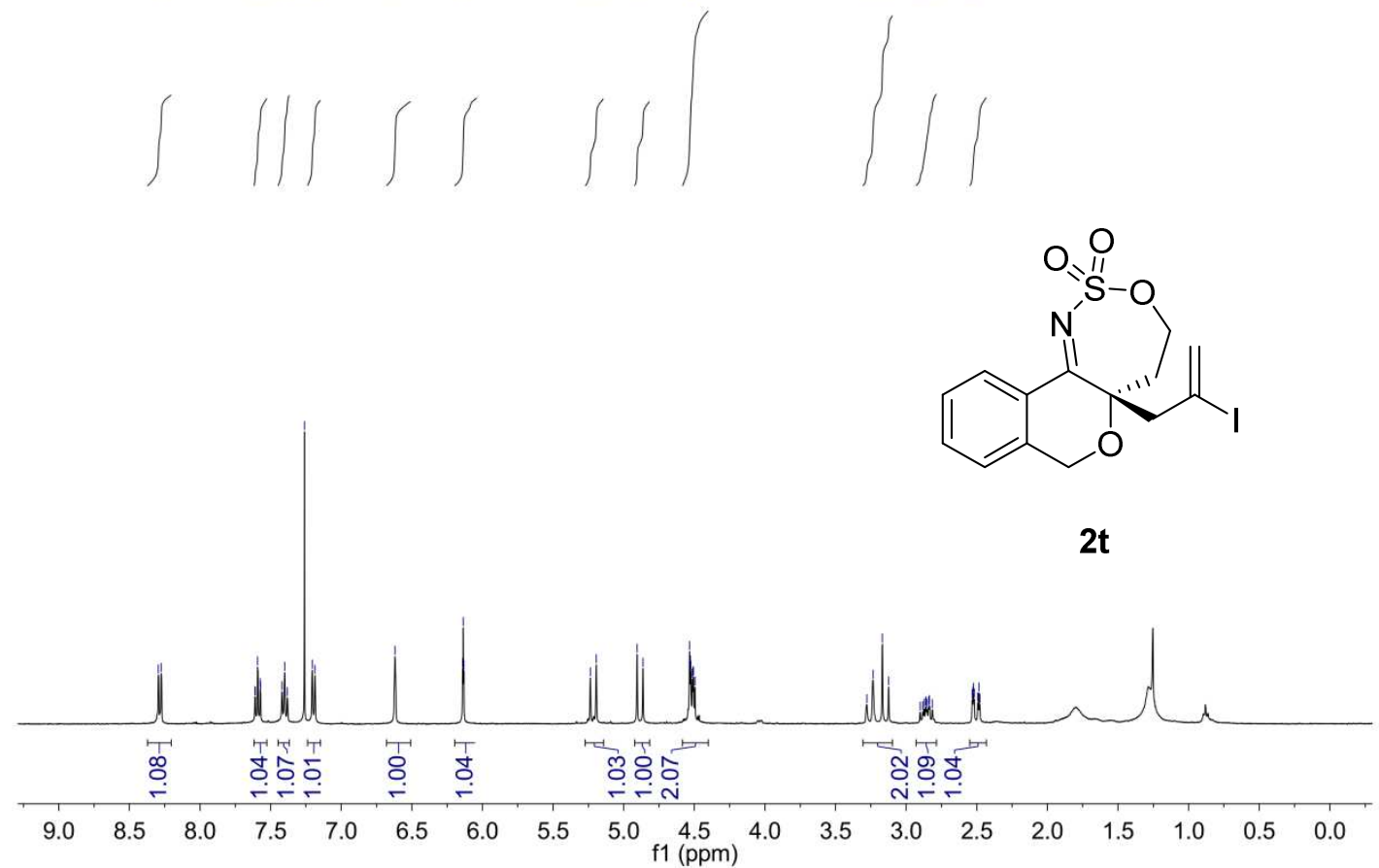


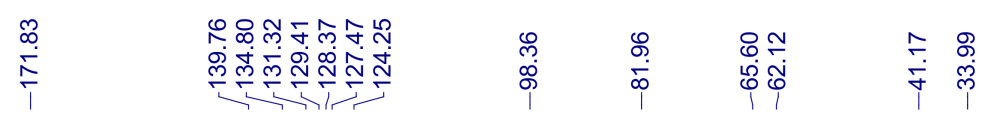

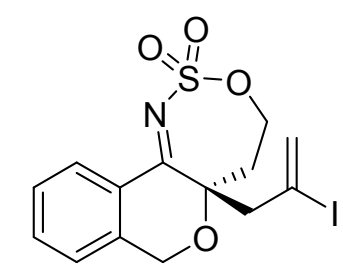

$2 t$

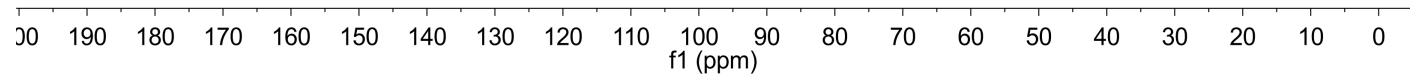

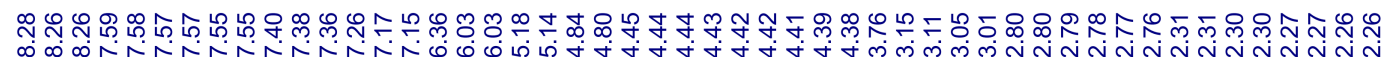

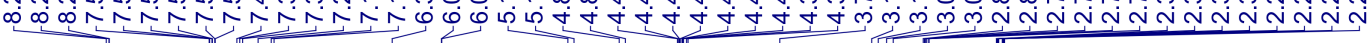

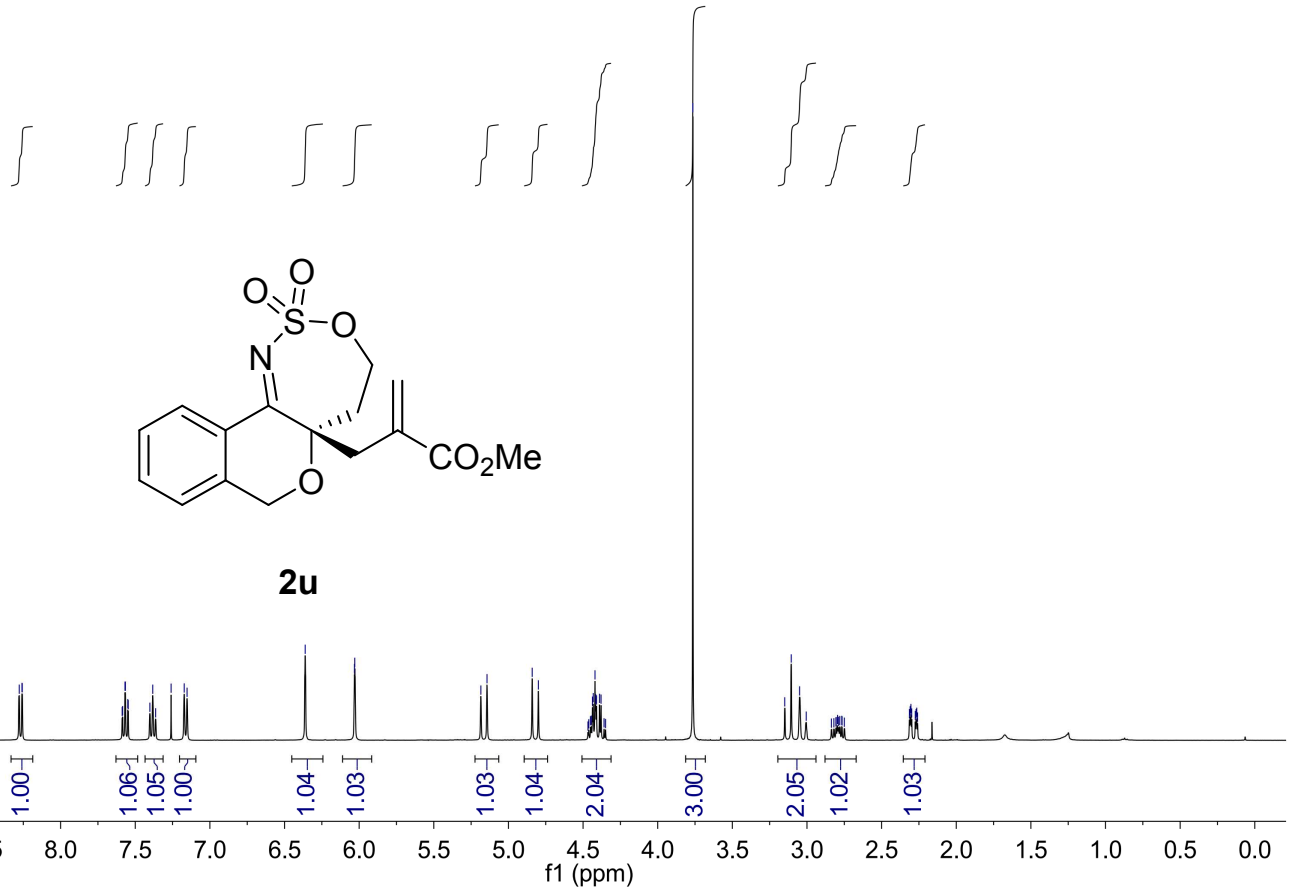




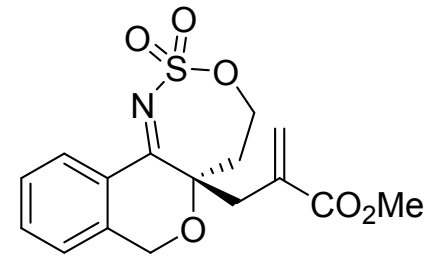

$2 u$
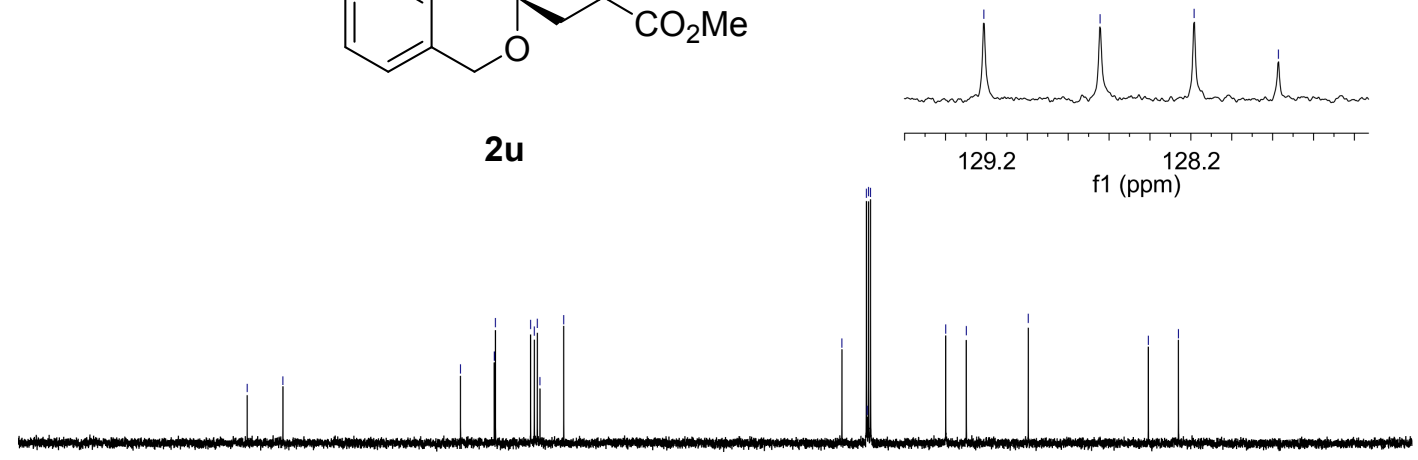

110 f1 (ppm) 90

$\begin{array}{lllllllll}80 & 70 & 60 & 50 & 40 & 30 & 20 & 10 & 0\end{array}$

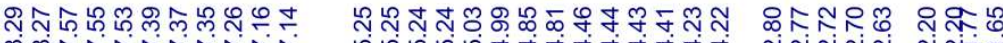

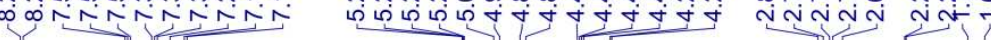
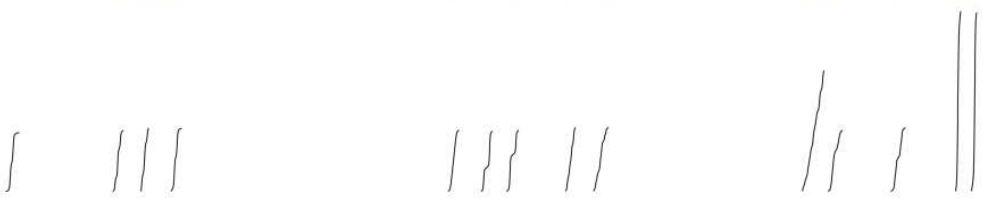<smiles>C=CC(C)(C)[C@@]12CCOS(=O)(=O)N=C1c1ccccc1CO2</smiles>

2v

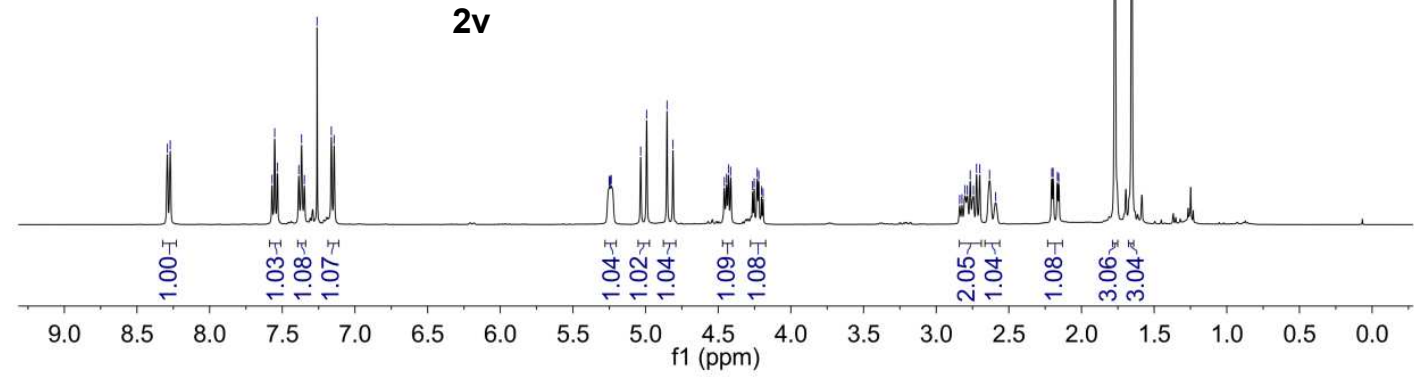



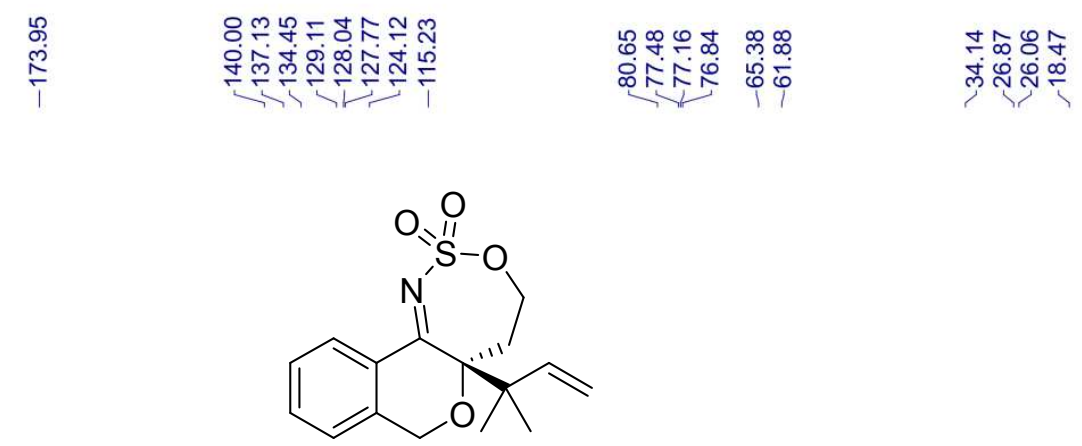

2v

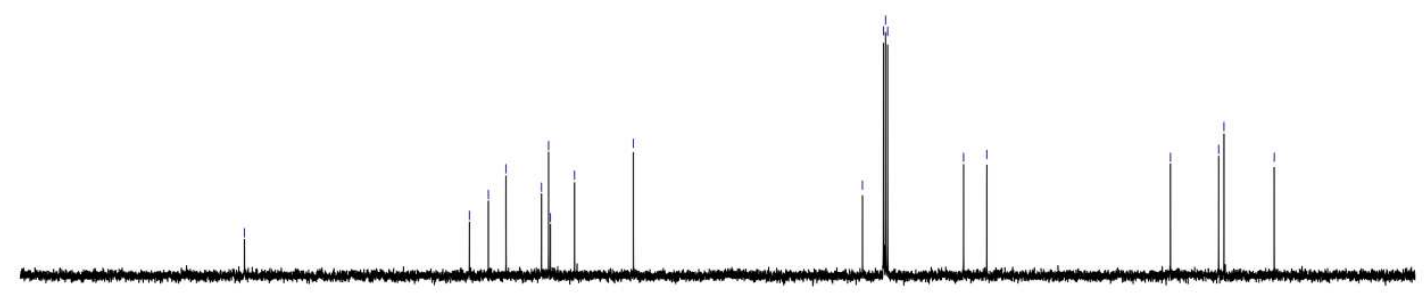

$\begin{array}{lllllllllllllllllllll}200 & 190 & 180 & 170 & 160 & 150 & 140 & 130 & 120 & \begin{array}{c}110 \\ \mathrm{f} 1(\mathrm{ppm})\end{array} & 100 & 90 & 80 & 70 & 60 & 50 & 40 & 30 & 20 & 10 & 0\end{array}$

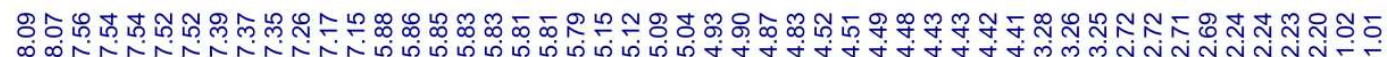

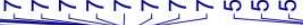

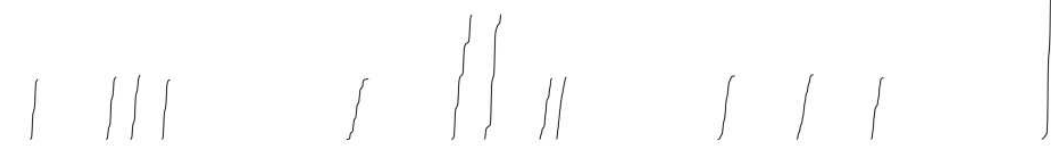<smiles>C=C[C@H](C)[C@]12CCOS(=O)(=O)N=C1c1ccccc1CO2</smiles>

$2 w$

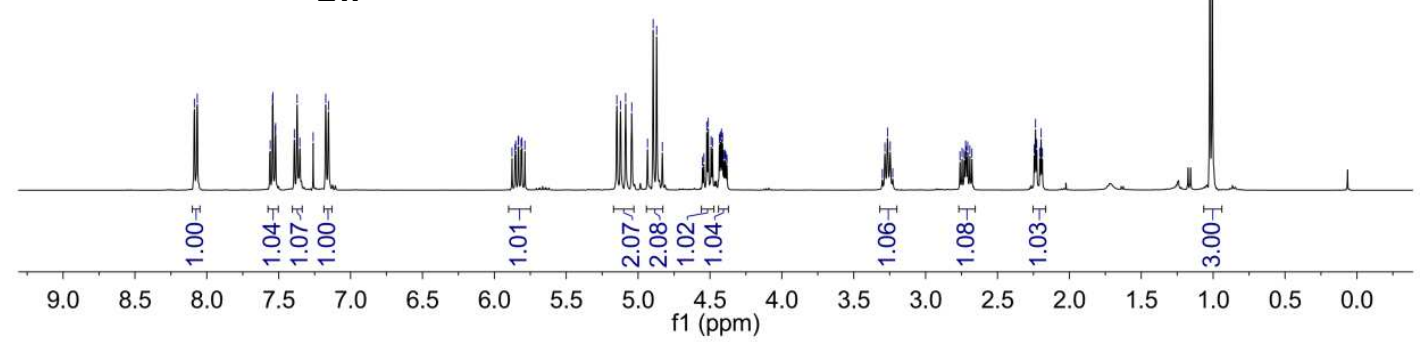




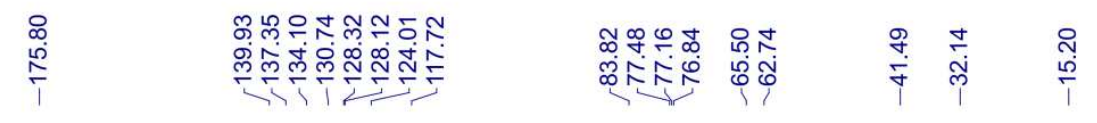<smiles>C=CC1(C)OCc2ccccc2C1=NS(=O)(=O)O</smiles>

$2 \mathrm{w}$

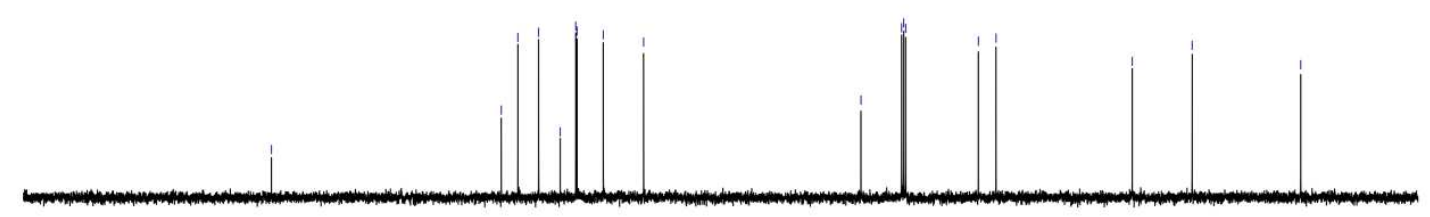

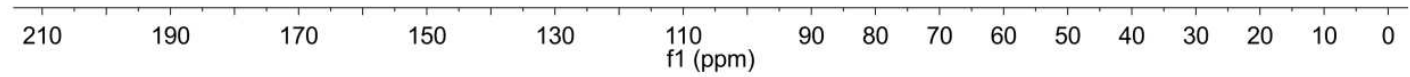

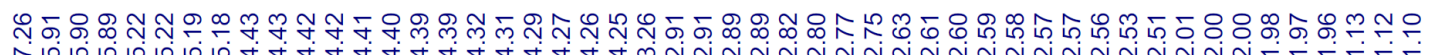
r.
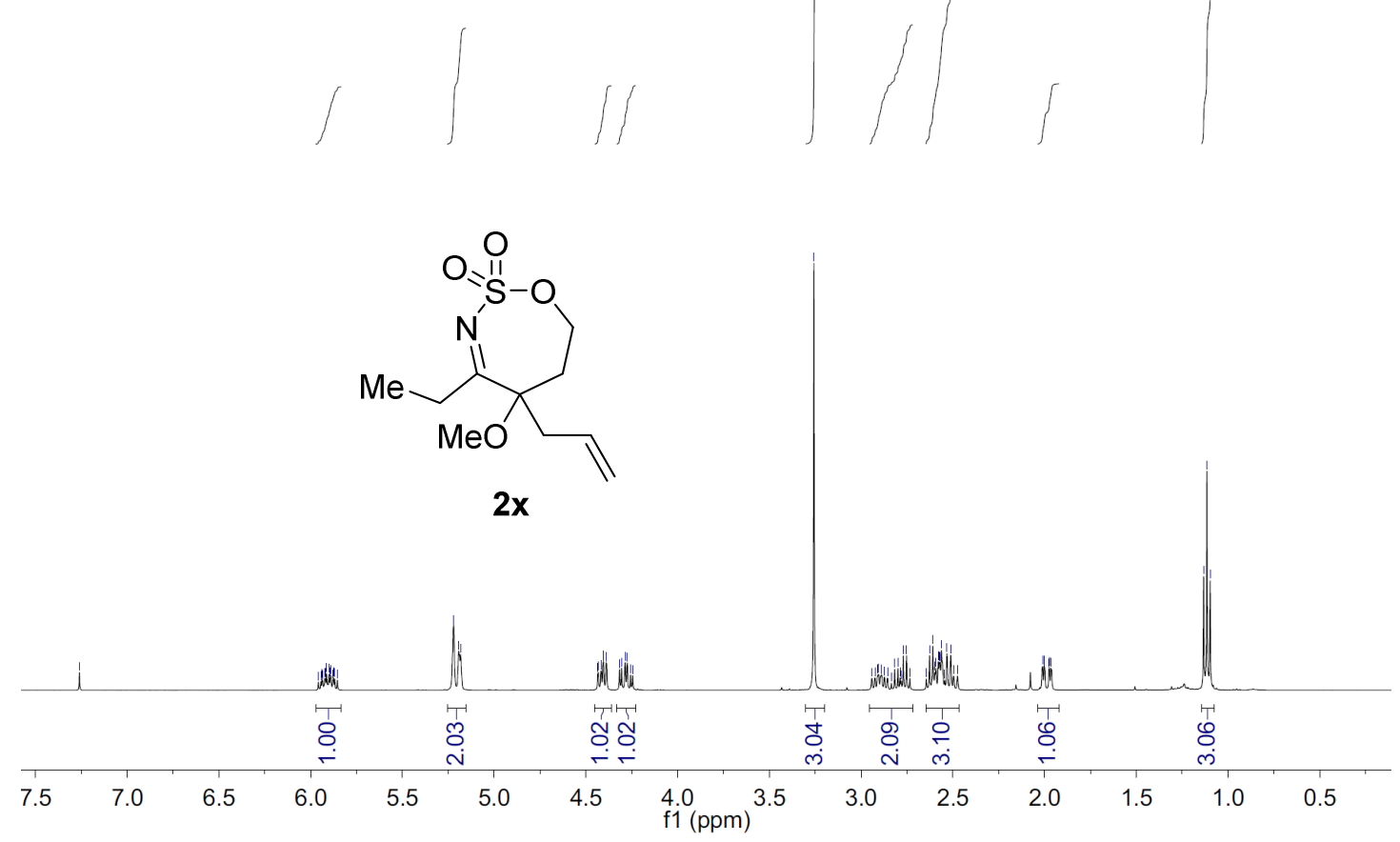


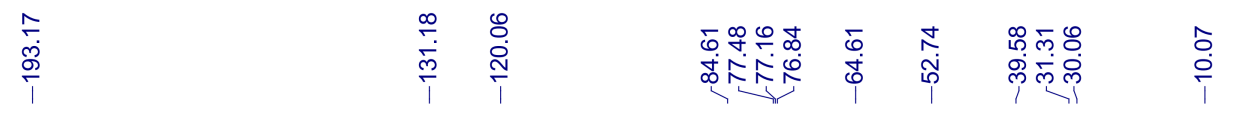
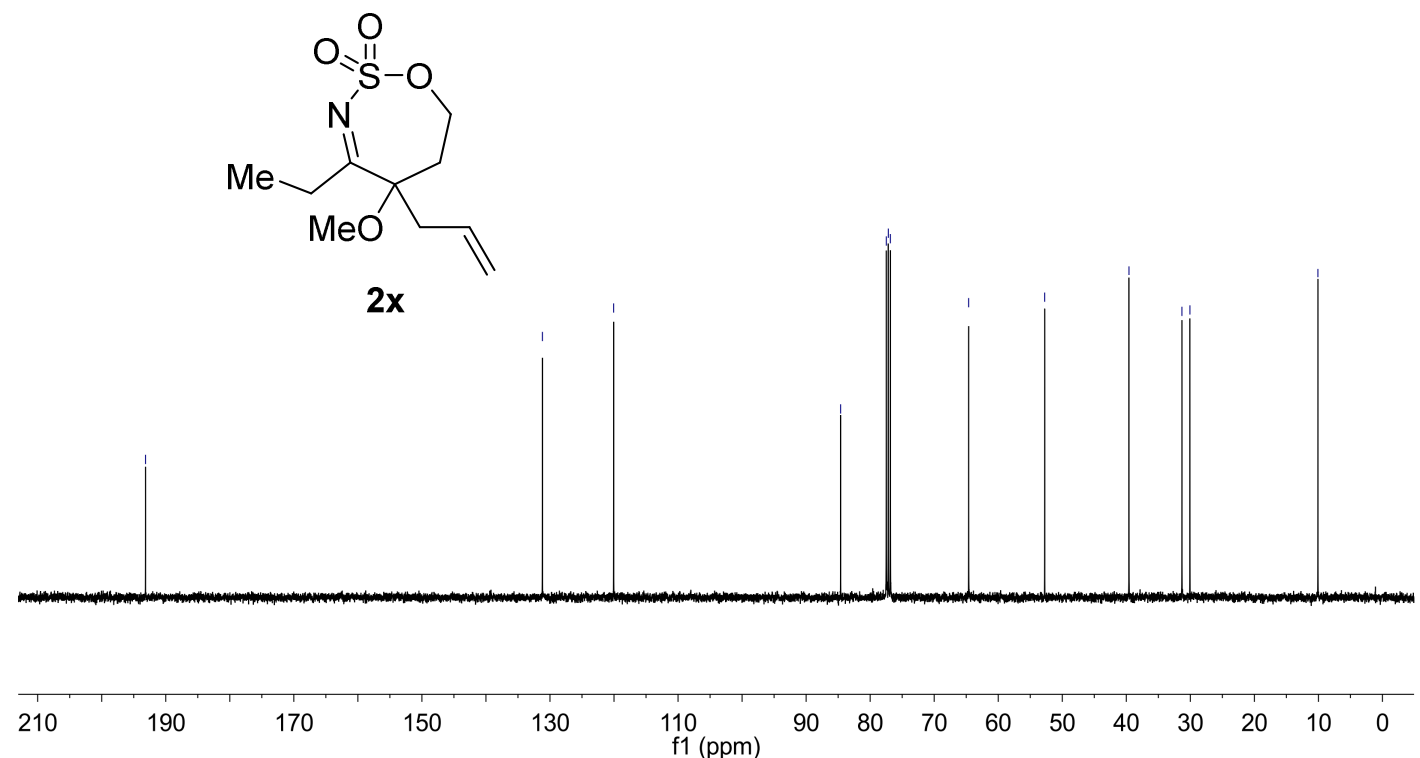

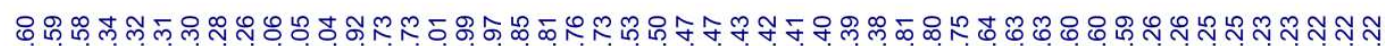

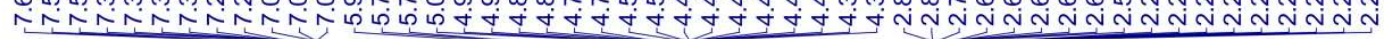
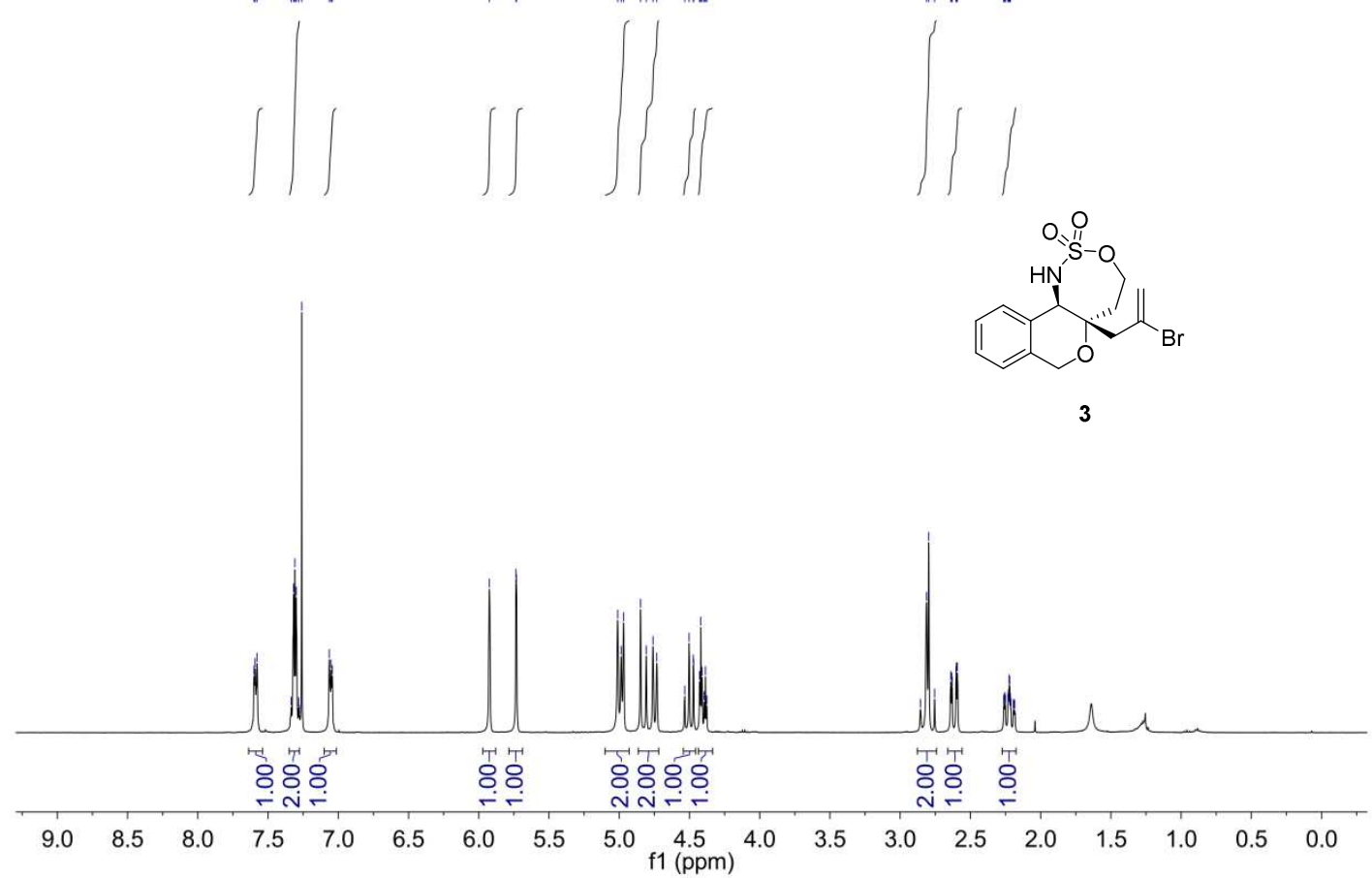

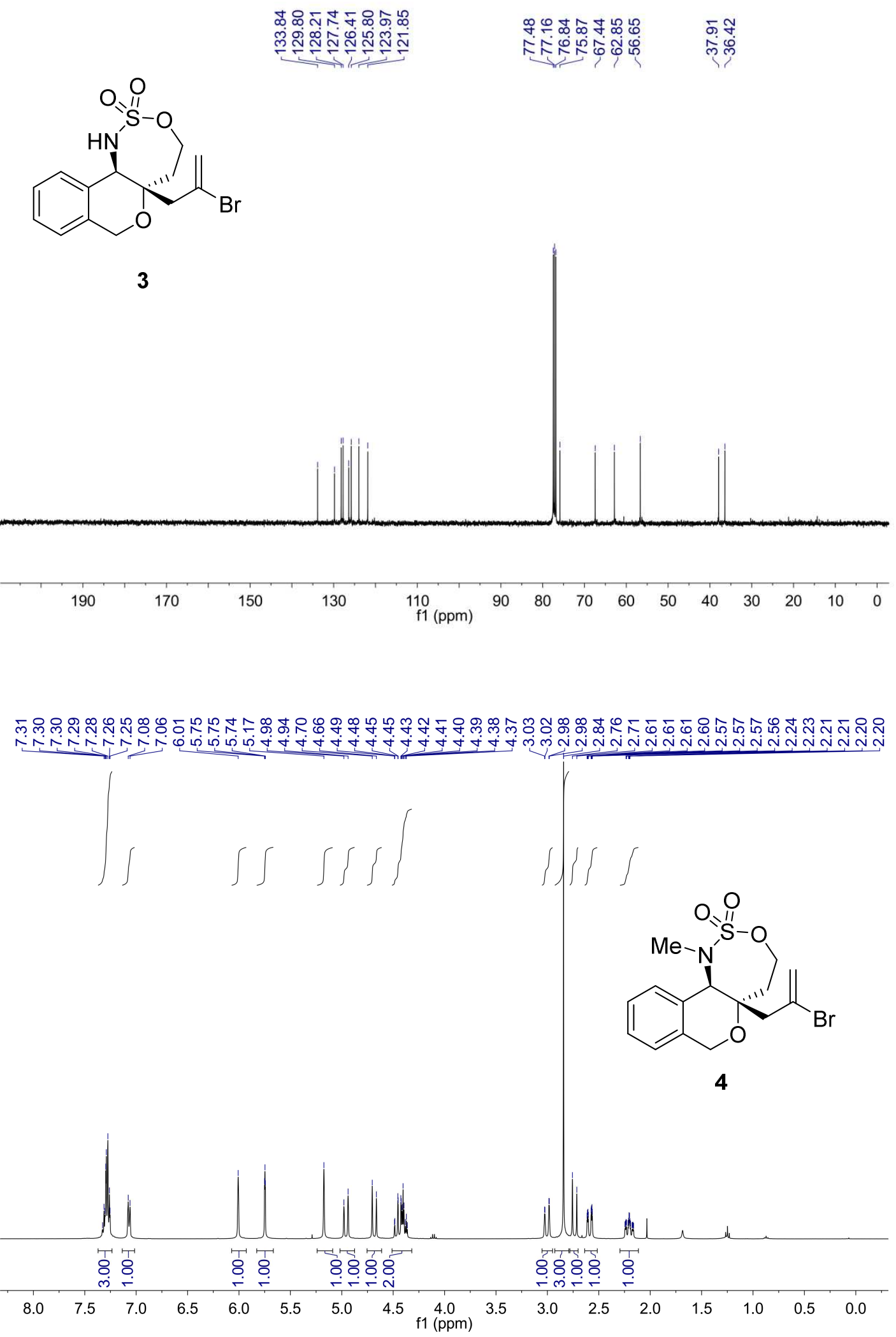


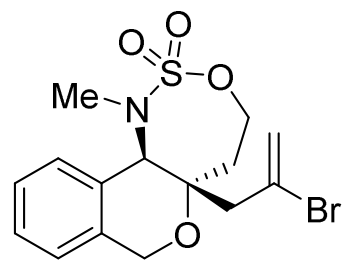

4

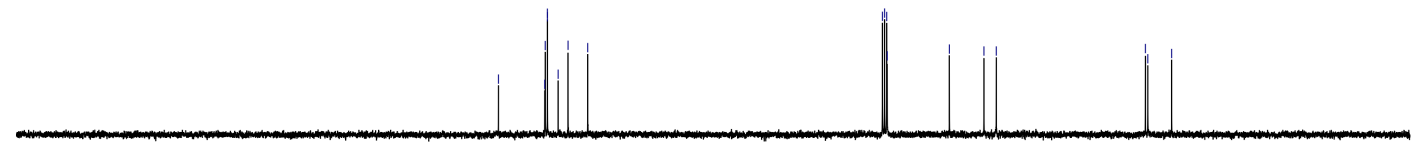

$\begin{array}{llllllllllllllllllll}200 & 190 & 180 & 170 & 160 & 150 & 140 & 130 & 120 & \underset{f}{110}\left(\begin{array}{ll}100 \\ \mathrm{f} 1(\mathrm{ppm})\end{array}\right. & 90 & 80 & 70 & 60 & 50 & 40 & 30 & 20 & 10 & 0\end{array}$

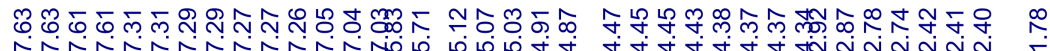

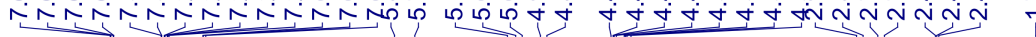
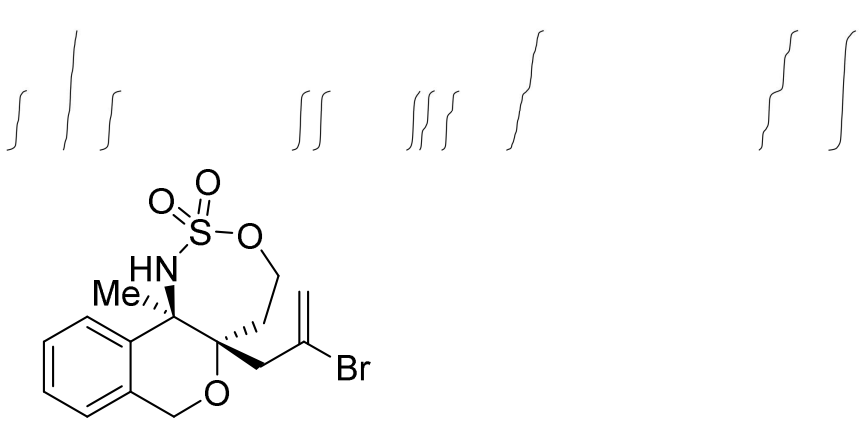

$5 s$

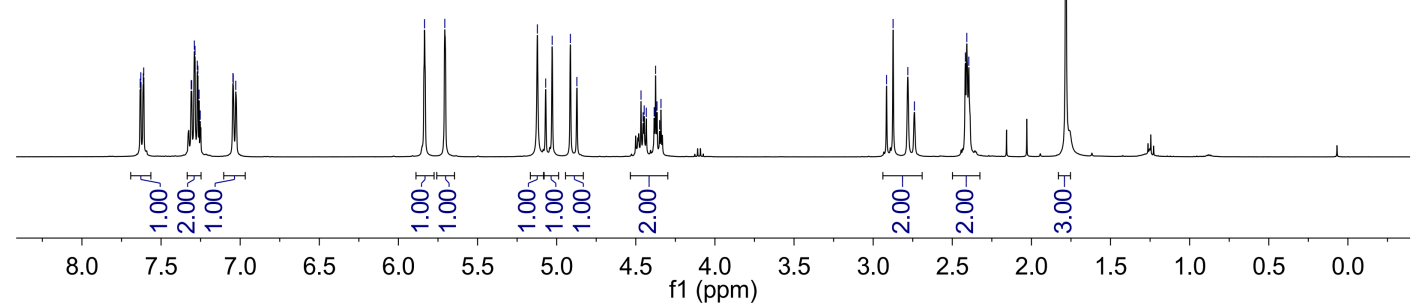




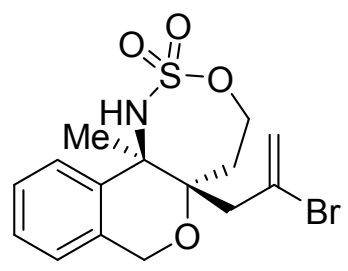

$5 s$

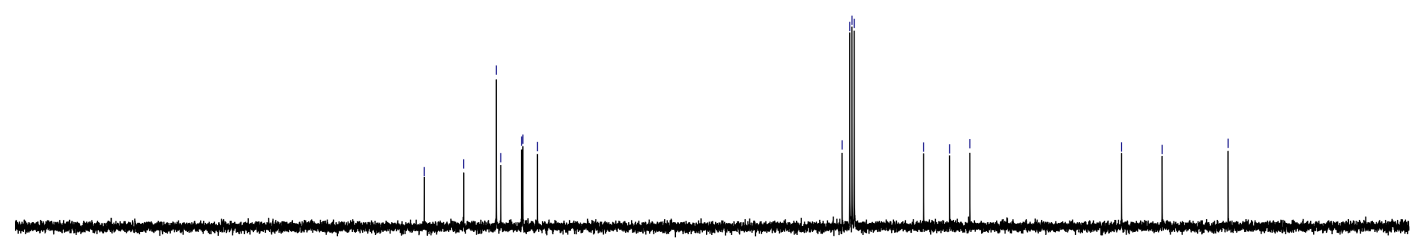

$\begin{array}{llllllllllllllllllll}190 & 180 & 170 & 160 & 150 & 140 & 130 & 120 & 110 & \begin{array}{c}100 \\ \mathrm{f} 1(\mathrm{ppm})\end{array} & 80 & 70 & 60 & 50 & 40 & 30 & 20 & 10 & 0\end{array}$

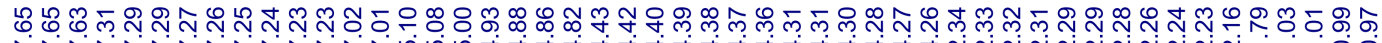

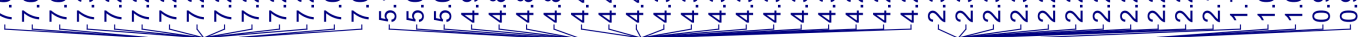

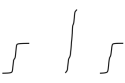<smiles>CC1C2C=CC=CC1CC2</smiles>

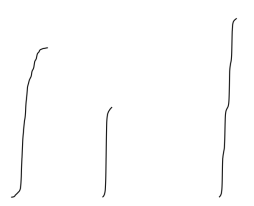<smiles>C=C(C[C@@]12CCOS(=O)(=O)N[C@]1(C)c1ccccc1CO2)C(C)C</smiles>

$5 q$

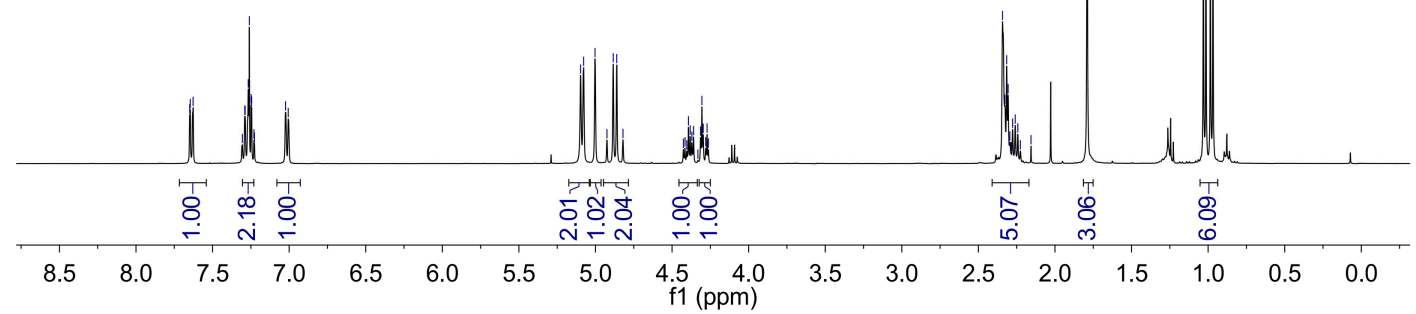




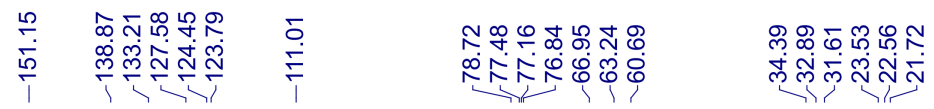<smiles>C=C(C[C@@]12CCOS(=O)(=O)N[C@]1(C)c1ccccc1CO2)C(C)C</smiles>

$5 q$

$\begin{array}{llllllllllllllllllll}200 & 190 & 180 & 170 & 160 & 150 & 140 & 130 & 120 & 110 \begin{array}{c}100 \\ \mathrm{f} 1(\mathrm{ppm})\end{array} & 90 & 80 & 70 & 60 & 50 & 40 & 30 & 20 & 10 & 0\end{array}$

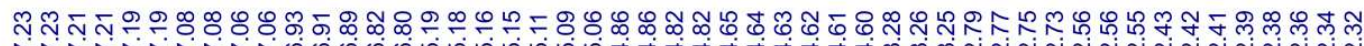

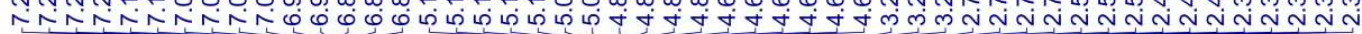
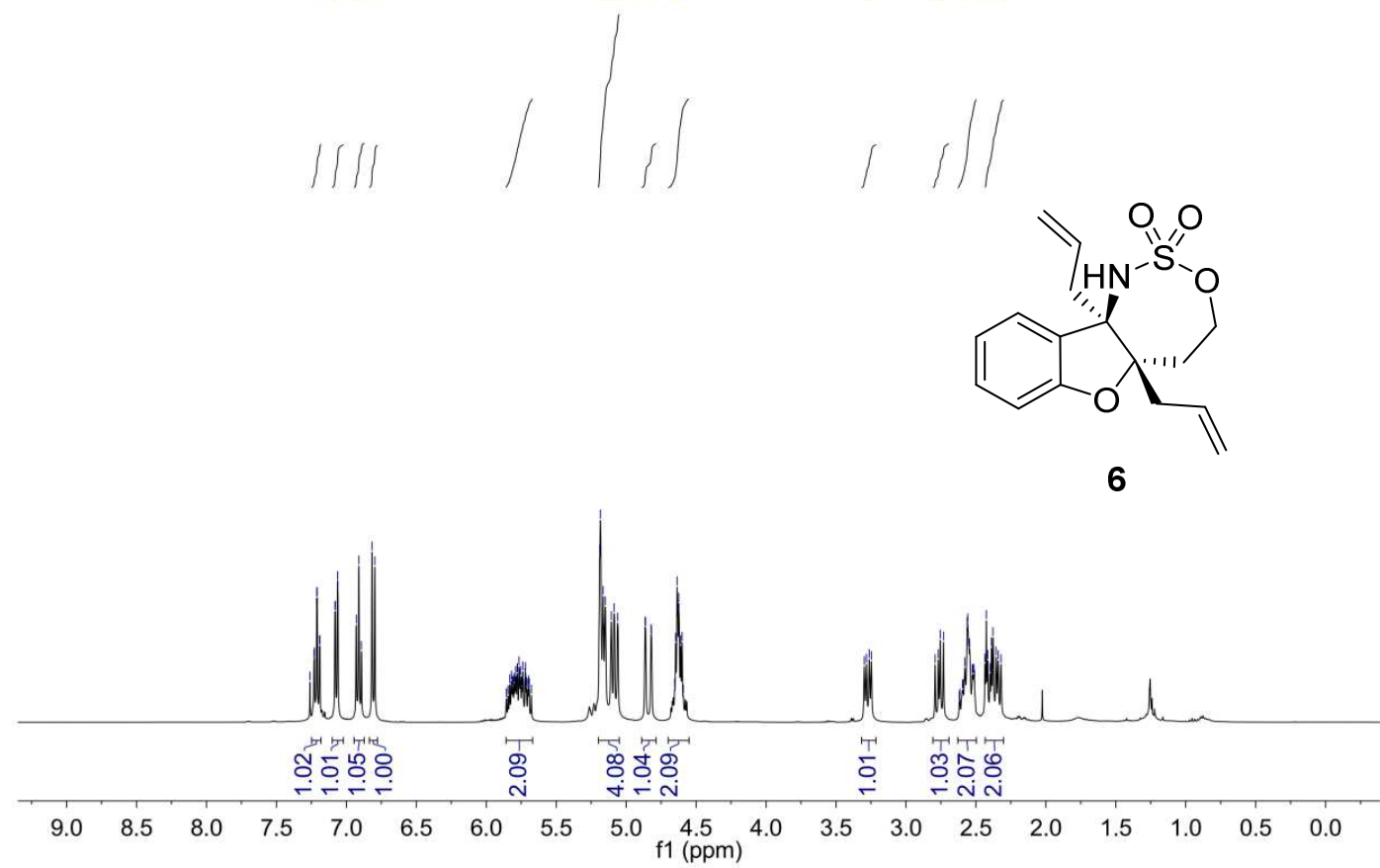


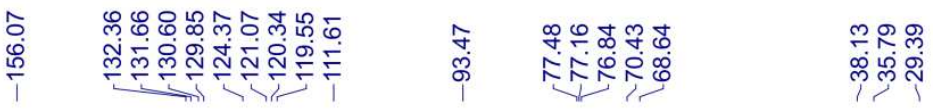<smiles>C=CC[C@]12NS(=O)(=O)OCC[C@]1(CC=C)c1ccccc1O2</smiles>

6

$\begin{array}{llllllllll}190 & 180 & 170 & 160 & 150 & 140 & 130 & 120 & 110 & 100 \\ \mathrm{f} 1(\mathrm{ppm})\end{array}$ $\begin{array}{lllllllll}80 & 70 & 60 & 50 & 40 & 30 & 20 & 10 & 0\end{array}$ 
Condition: hexane $:$ 2-propanol $=90: 10$

Flow rate $=1.0 \mathrm{~mL} / \mathrm{min}, \lambda=254 \mathrm{~nm}$, Daicel Chiralcel IA

\section{$<$ Chromatogram $>$}

$\mathrm{mAU}$

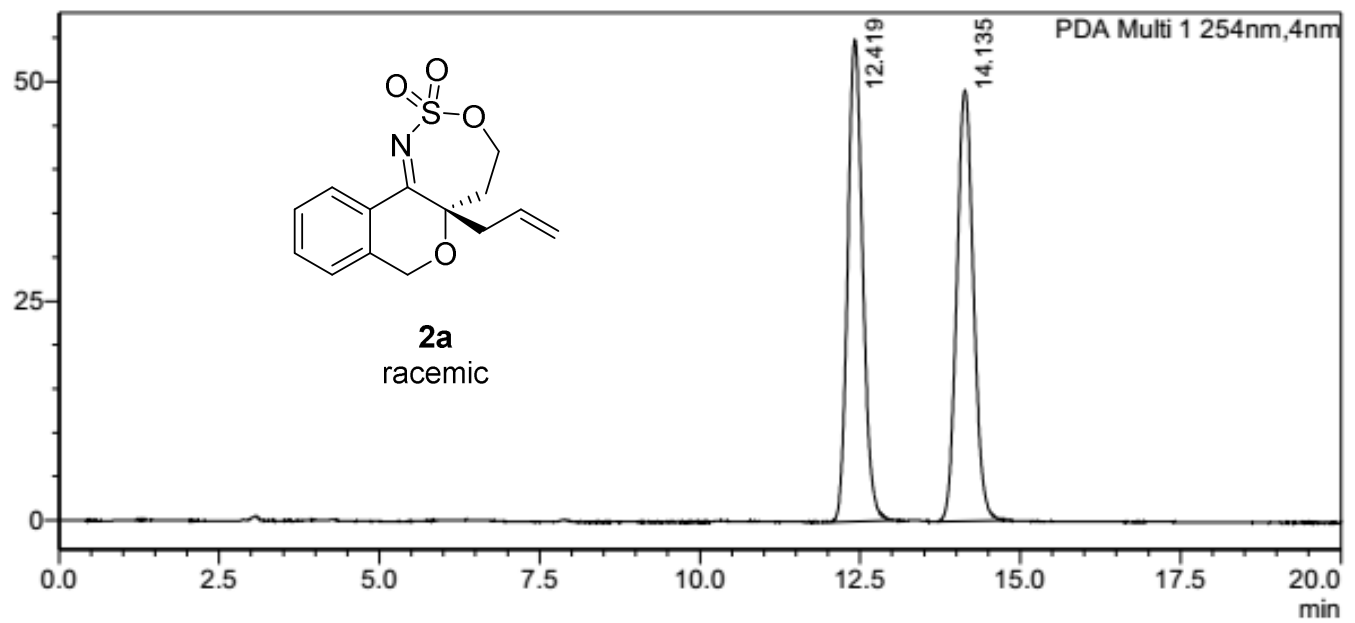

<Peak Table>

\section{PDA Ch1 254nm}

\begin{tabular}{|c|c|c|c|c|}
\hline Peak\# & Ret. Time & Area & Height & Area $\%$ \\
\hline 1 & 12.419 & 895069 & 54883 & 50.025 \\
\hline 2 & 14.135 & 894164 & 49101 & 49.975 \\
\hline Total & & 1789233 & 103984 & 100.000 \\
\hline
\end{tabular}

$<$ Chromatogram>

mAU

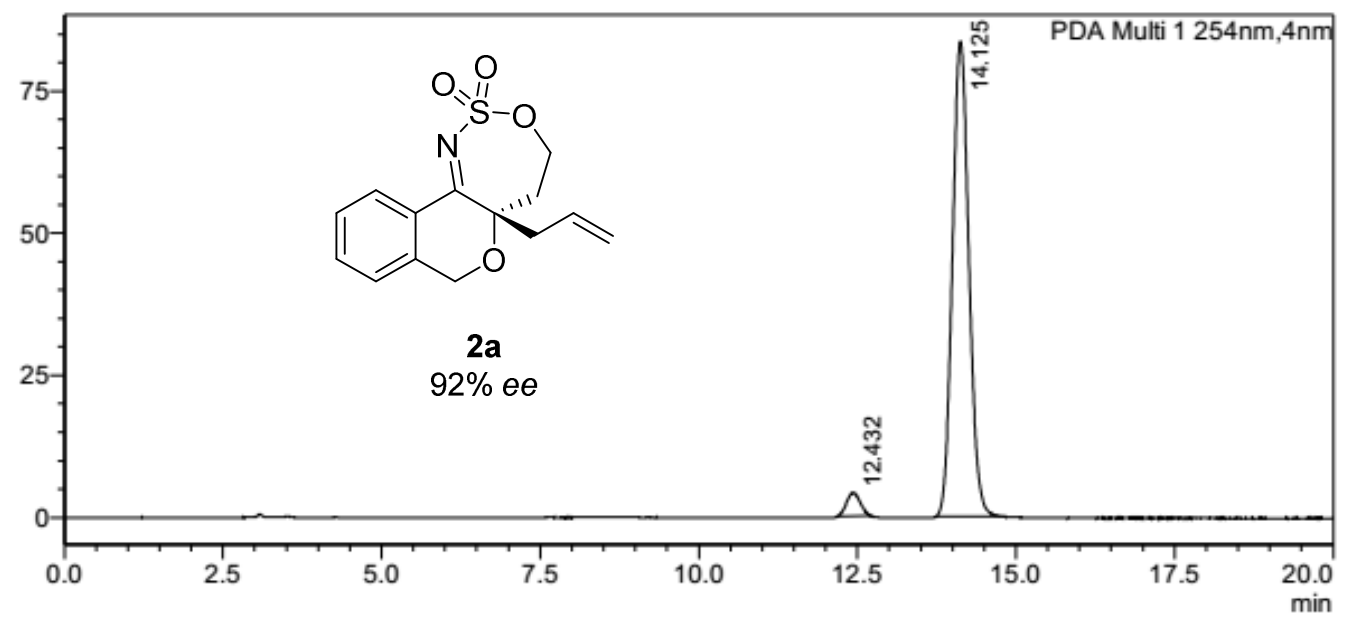

<Peak Table>

PDA Ch1 254nm

\begin{tabular}{|c|c|c|c|c|}
\hline Peak\# & Ret. Time & Area & Height & Area $\%$ \\
\hline 1 & 12.432 & 62283 & 4124 & 3.966 \\
\hline 2 & 14.125 & 1508171 & 83505 & 96.03 \\
\hline Tota & & 1570454 & 87629 & \\
\hline
\end{tabular}


Condition: hexane $:$ 2-propanol $=90: 10$

Flow rate $=1.0 \mathrm{~mL} / \mathrm{min}, \lambda=254 \mathrm{~nm}$, Daicel Chiralcel IA

\section{$<$ Chromatogram>}

mAU

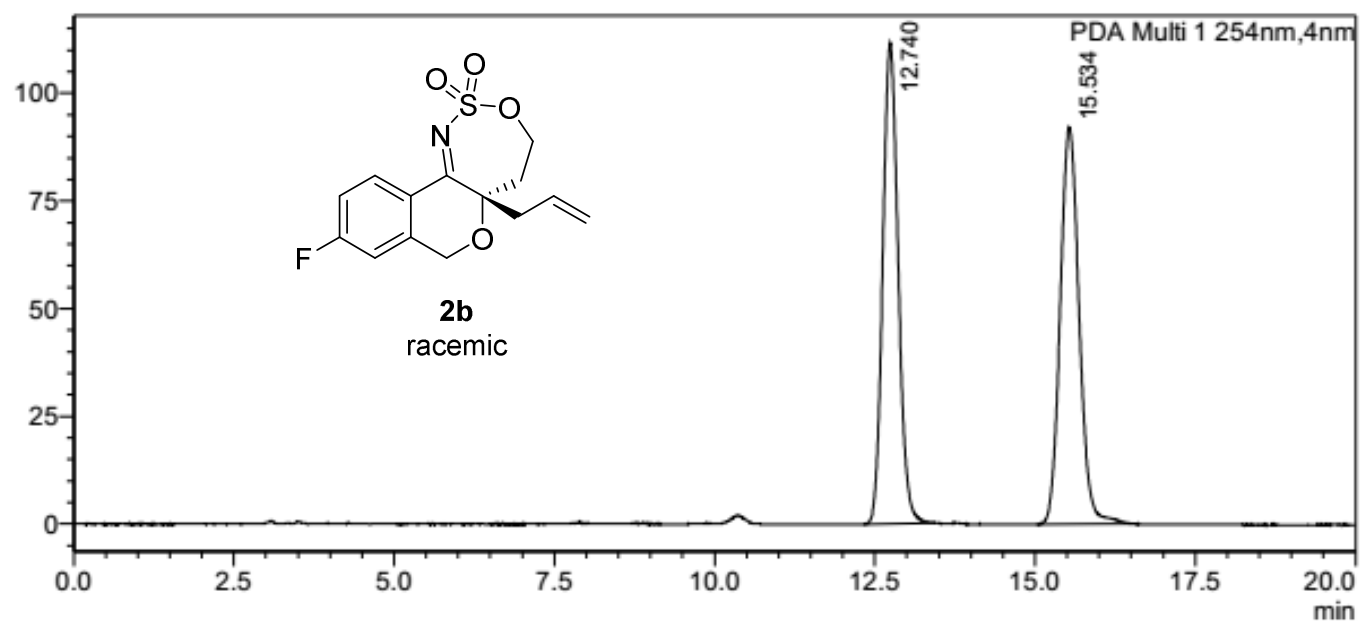

<Peak Table>

PDA Ch1 254nm

\begin{tabular}{|l|l|l|l|}
\hline Peak\# Ret. Time & Area & Height & Area \% \\
\hline 1 & &
\end{tabular}

\begin{tabular}{|c|c|c|c|c|}
\hline & . & & & . \\
\hline 1 & 12.740 & 1870191 & 111701 & 49.620 \\
\hline 2 & 15.534 & 1898825 & 92163 & 50.380 \\
\hline Total & & 3769016 & 203864 & 100.000 \\
\hline
\end{tabular}

\section{$<$ Chromatogram $>$}

mAU

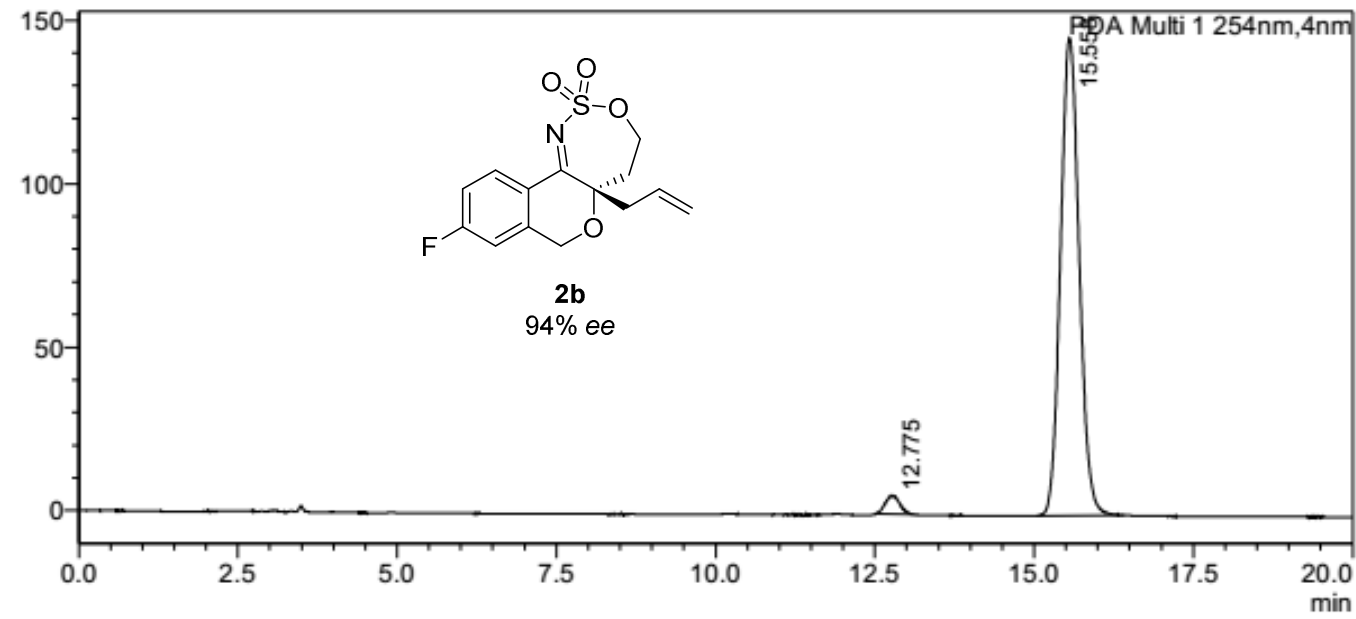

<Peak Table>

PDA Ch1 254nm

\begin{tabular}{|r|r|r|r|r|}
\hline Peak\# & Ret. Time & \multicolumn{1}{c|}{ Area } & \multicolumn{1}{c|}{ Height } & \multicolumn{1}{c|}{ Area\% } \\
\hline 1 & 12.775 & 89872 & 5668 & 2.924 \\
\hline 2 & 15.558 & 2983779 & 146041 & 97.076 \\
\hline Total & & 3073651 & 151709 & 100.000 \\
\hline
\end{tabular}


Condition: hexane $:$ 2-propanol $=90: 10$

Flow rate $=1.0 \mathrm{~mL} / \mathrm{min}, \lambda=254 \mathrm{~nm}$, Daicel Chiralcel IA

<Chromatogram>

mAU

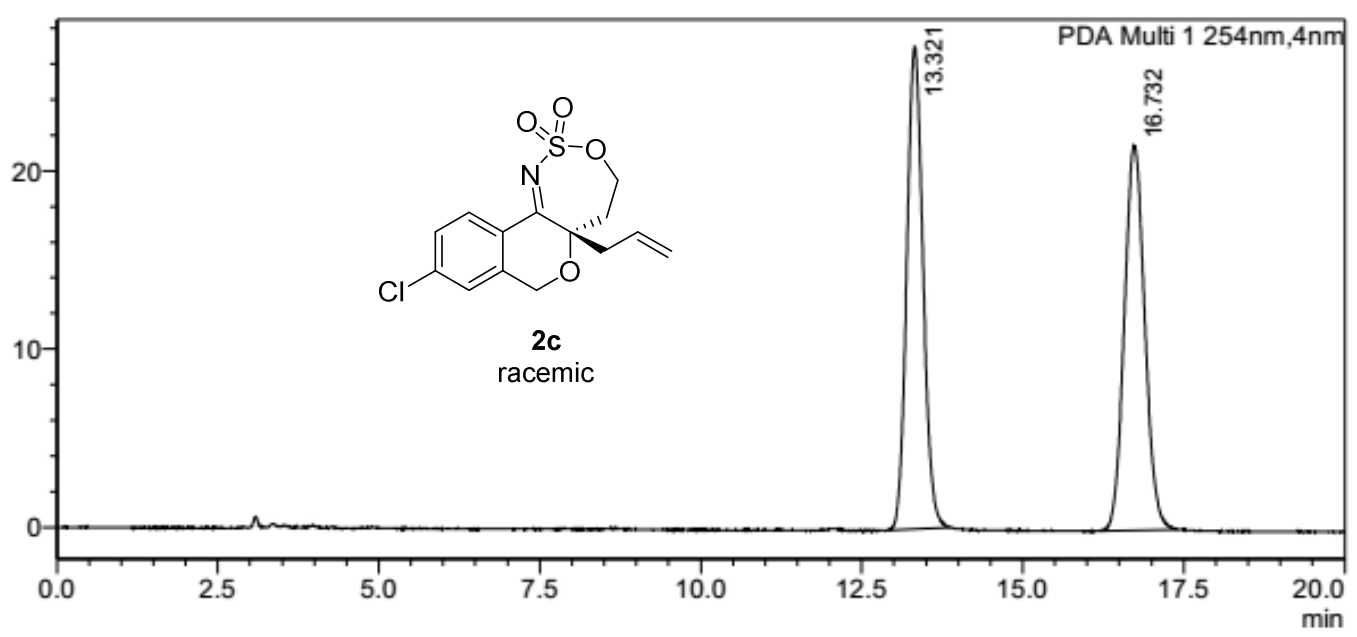

<Peak Table>

PDA Ch1 254nm

\begin{tabular}{|c|c|c|c|c|}
\hline Peak\# & Ret. Time & Area & Height & Area\% \\
\hline 1 & 13.321 & 474304 & 27070 & 49.843 \\
\hline 2 & 16.732 & 477295 & 21606 & 50.157 \\
\hline Total & & 951599 & 48677 & 100.000 \\
\hline
\end{tabular}

$<$ Chromatogram>

mAU

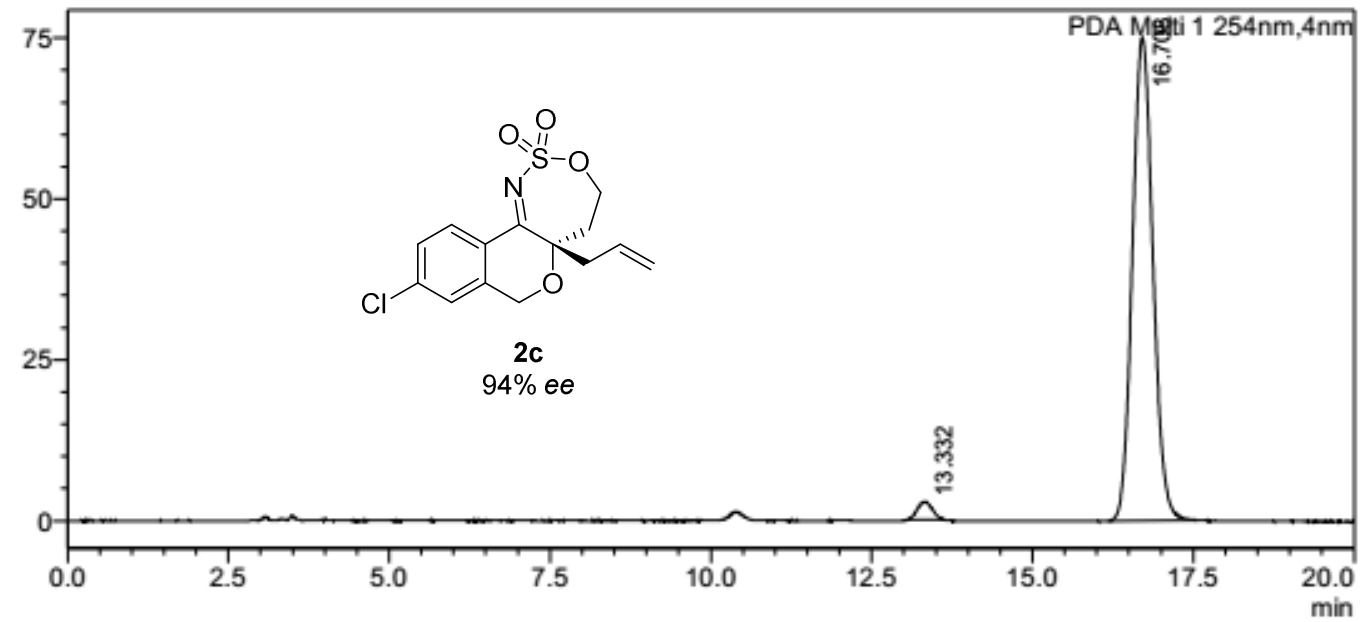

<Peak Table>

PDA Ch1 254nm

\begin{tabular}{|r|r|r|r|r|}
\hline Peak\# & Ret. Time & \multicolumn{1}{c|}{ Area } & Height & \multicolumn{1}{c|}{ Area\% } \\
\hline 1 & 13.332 & 44998 & 2796 & 2.631 \\
\hline 2 & 16.708 & 1665113 & 75113 & 97.369 \\
\hline Total & & 1710111 & 77908 & 100.000 \\
\hline
\end{tabular}


Condition: hexane $:$-propanol $=95: 5$

Flow rate $=1.0 \mathrm{~mL} / \mathrm{min}, \lambda=254 \mathrm{~nm}$, Daicel Chiralpak AD-H

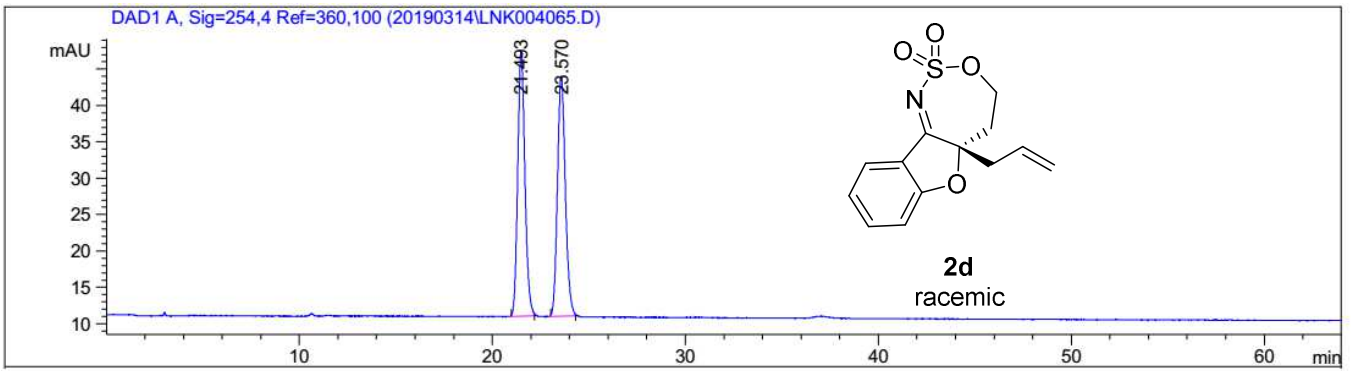

Signal 1: DAD1 A, Sig=254,4 Ref $=360,100$

\begin{tabular}{|c|c|c|c|c|c|c|}
\hline $\begin{array}{c}\text { Peak } \\
\#\end{array}$ & $\begin{array}{c}\text { RetTime } \\
\text { [min] }\end{array}$ & Type & $\begin{array}{c}\text { Width } \\
\text { [min] }\end{array}$ & $\begin{array}{c}\text { Area } \\
{\left[\mathrm{mAU}{ }^{\star} \mathrm{s}\right]}\end{array}$ & $\begin{array}{l}\text { Height } \\
\text { [mAU] }\end{array}$ & $\begin{array}{c}\text { Area } \\
\frac{\circ}{\delta}\end{array}$ \\
\hline & 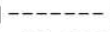 & & ----- & |-------- & $---\infty--$ & ----- \\
\hline 1 & 21.493 & $\mathrm{BB}$ & 0.3991 & 942.69385 & 36.28212 & 50.1592 \\
\hline 2 & 23.570 & BB & 0.4353 & 936.70862 & 32.98335 & 49.8408 \\
\hline \multicolumn{4}{|c|}{ Totals : } & 1879.40247 & 69.26546 & \\
\hline
\end{tabular}

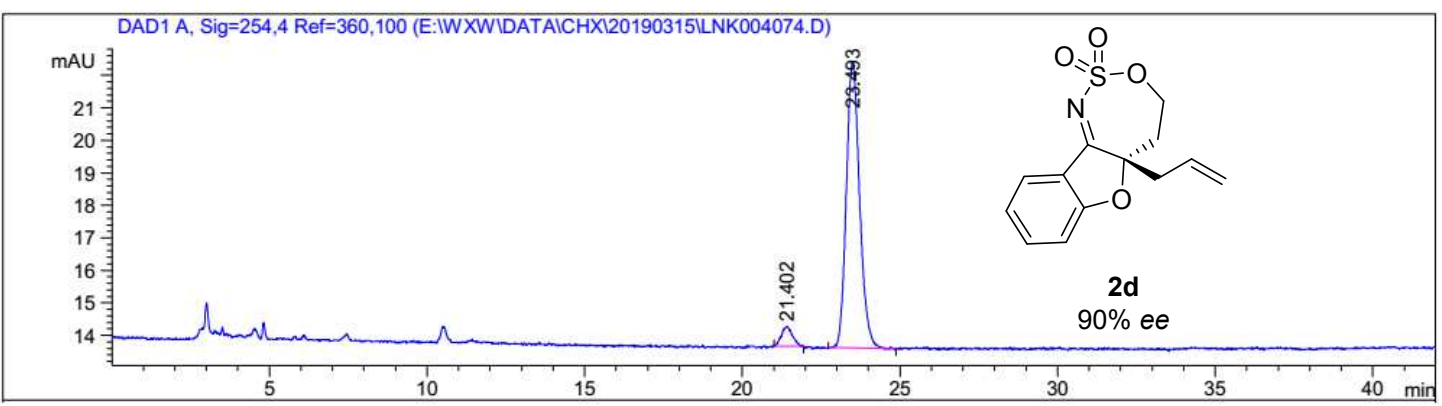

Signal 1: DAD1 A, Sig=254, 4 Ref $=360,100$

\begin{tabular}{|c|c|c|c|c|c|c|}
\hline $\begin{array}{c}\text { Peak } \\
\#\end{array}$ & $\begin{array}{c}\text { RetTime } \\
\text { [min] }\end{array}$ & Type & $\begin{array}{l}\text { Width } \\
\text { [min] }\end{array}$ & $\begin{array}{c}\text { Area } \\
{\left[\mathrm{mAU}^{\star} \mathrm{s}\right]}\end{array}$ & $\begin{array}{l}\text { Height } \\
\text { [mAU] }\end{array}$ & $\begin{array}{c}\text { Area } \\
\frac{8}{6}\end{array}$ \\
\hline \multicolumn{7}{|c|}{ 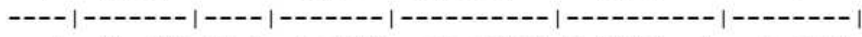 } \\
\hline 1 & 21.402 & MM R & 0.4110 & 14.92761 & $6.05281 e-1$ & 5.4905 \\
\hline 2 & 23.493 & MM R & 0.4876 & 256.95197 & 8.78244 & 94.5095 \\
\hline ota & $1 \mathrm{~s}:$ & & & 271.87958 & 9.38772 & \\
\hline
\end{tabular}




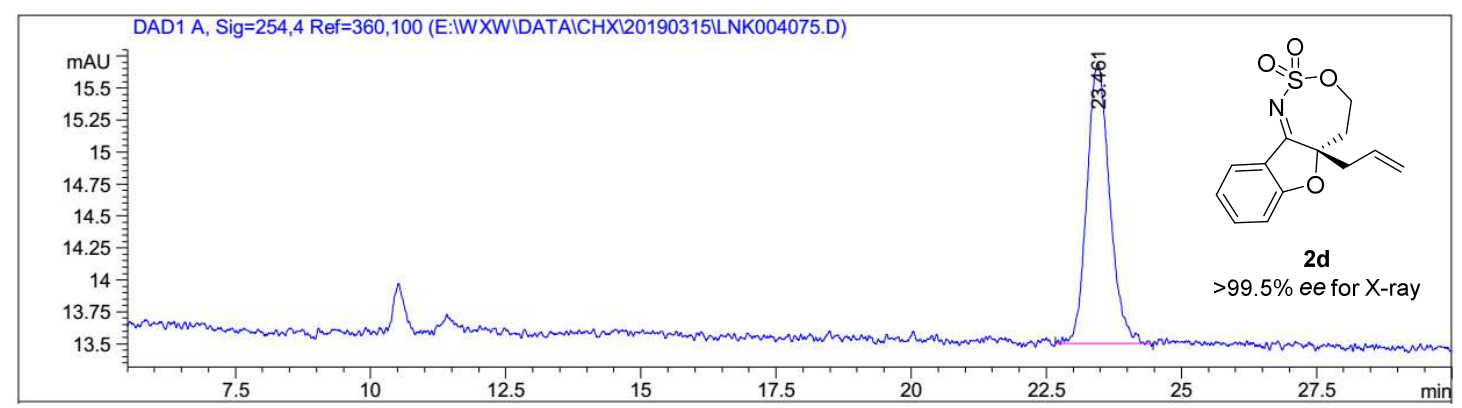

Signal 1: DAD1 A, Sig=254, 4 Ref $=360,100$

\begin{tabular}{|c|c|c|c|c|c|}
\hline $\begin{array}{c}\text { Peak } \\
\#\end{array}$ & $\begin{array}{l}\text { RetTime Type } \\
\text { [min] }\end{array}$ & $\begin{array}{l}\text { Width } \\
\text { [min] }\end{array}$ & $\begin{array}{c}\text { Area } \\
{\left[\mathrm{mAU}{ }^{\star} \mathrm{s}\right]}\end{array}$ & $\begin{array}{l}\text { Height } \\
\text { [mAU] }\end{array}$ & $\begin{array}{c}\text { Area } \\
\quad \frac{\circ}{6}\end{array}$ \\
\hline \multicolumn{6}{|c|}{$----|-------|$} \\
\hline 1 & $23.461 \mathrm{MM} \mathrm{R}$ & 0.4900 & 64.23614 & 2.18468 & 100.0000 \\
\hline Total & S : & & 64.23614 & 2.18468 & \\
\hline
\end{tabular}

Condition: hexane $:$-propanol $=90: 10$

Flow rate $=1.0 \mathrm{~mL} / \mathrm{min}, \lambda=254 \mathrm{~nm}$, Daicel Chiralcel IA

\section{$<$ Chromatogram $>$}

mAU

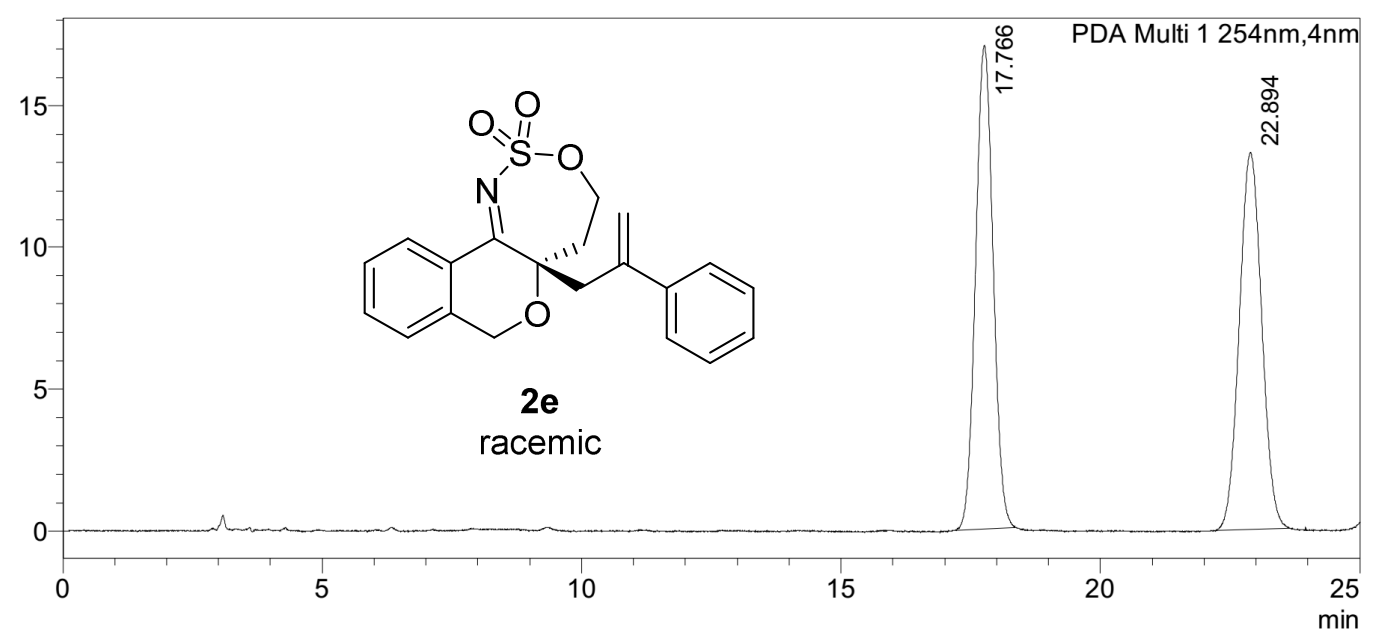

<Peak Table>

PDA Ch1 254nm

\begin{tabular}{|r|r|r|r|r|}
\hline Peak\# & Ret. Time & Area & Height & \multicolumn{1}{|c|}{ Area\% } \\
\hline 1 & 17.766 & 400296 & 17039 & 50.079 \\
\hline 2 & 22.894 & 399033 & 13286 & 49.921 \\
\hline Total & & 799329 & 30324 & 100.000 \\
\hline
\end{tabular}




\section{<Chromatogram>}

mAU

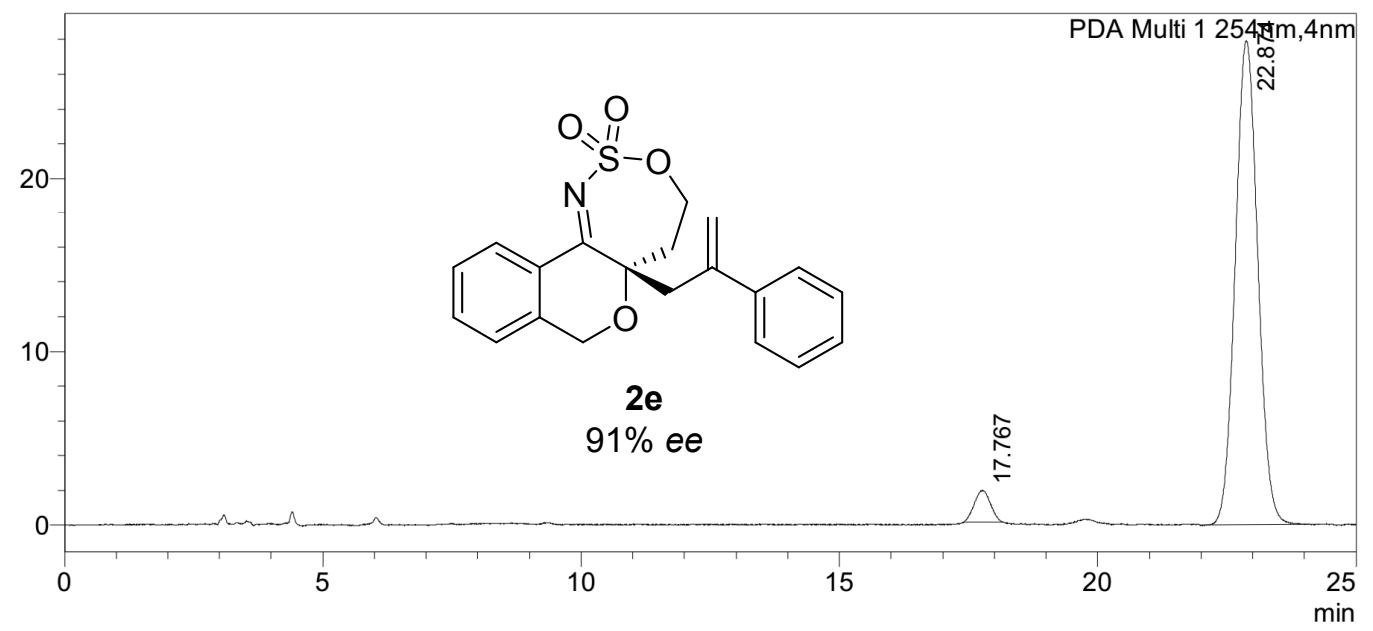

<Peak Table>

PDA Ch1 254nm

\begin{tabular}{|r|r|r|r|r|}
\hline Peak\# Ret. Time & \multicolumn{1}{|c|}{ Area } & Height & \multicolumn{1}{|c|}{ Area\% } \\
\hline 1 & 17.767 & 40367 & 1826 & 4.561 \\
\hline 2 & 22.874 & 844601 & 27892 & 95.439 \\
\hline Total & & 884968 & 29718 & 100.000 \\
\hline
\end{tabular}

Condition: hexane : 2-propanol $=90: 10$

Flow rate $=1.0 \mathrm{~mL} / \mathrm{min}, \lambda=254 \mathrm{~nm}$, Daicel Chiralcel IA

\section{$<$ Chromatogram>}

mAU

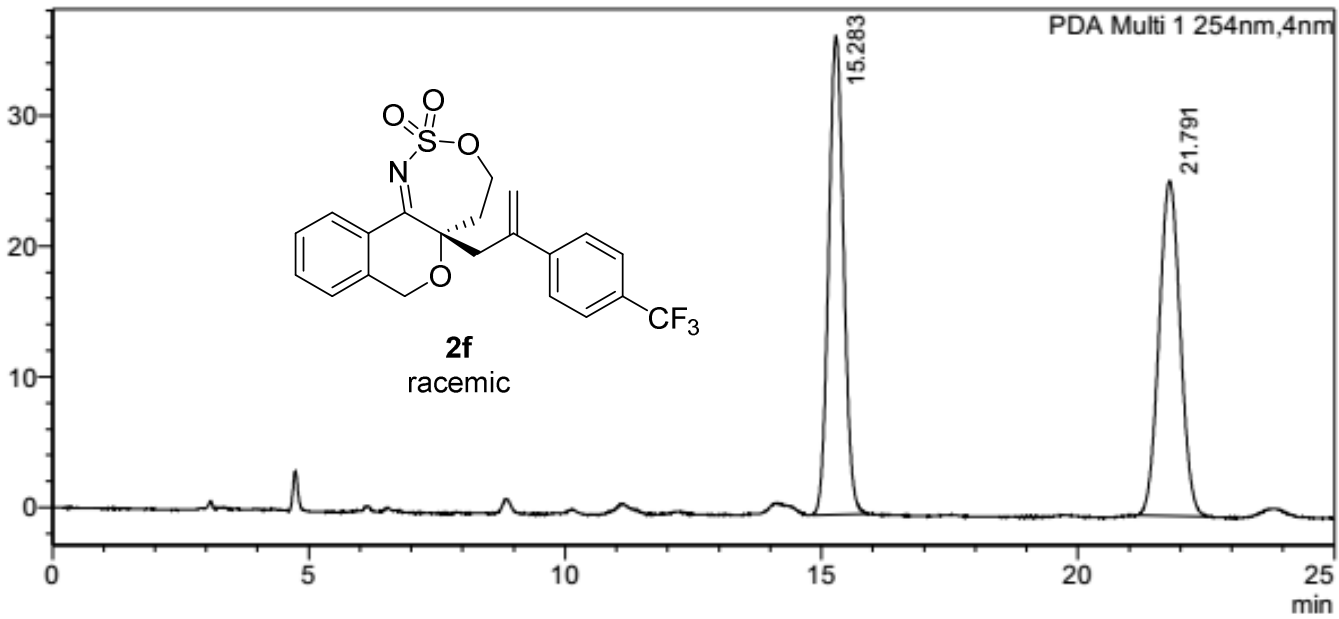

<Peak Table>

PDA Ch1 254nm

\begin{tabular}{|r|r|r|r|r|}
\hline Peak\#\# & Ret. Time & \multicolumn{1}{|c|}{ Area } & Height & \multicolumn{1}{c|}{ Area\% } \\
\hline 1 & 15.283 & 744880 & 36597 & 50.280 \\
\hline 2 & 21.791 & 736580 & 25613 & 49.720 \\
\hline Total & & 1481460 & 62210 & 100.000 \\
\hline
\end{tabular}


$<$ Chromatogram>

mAU

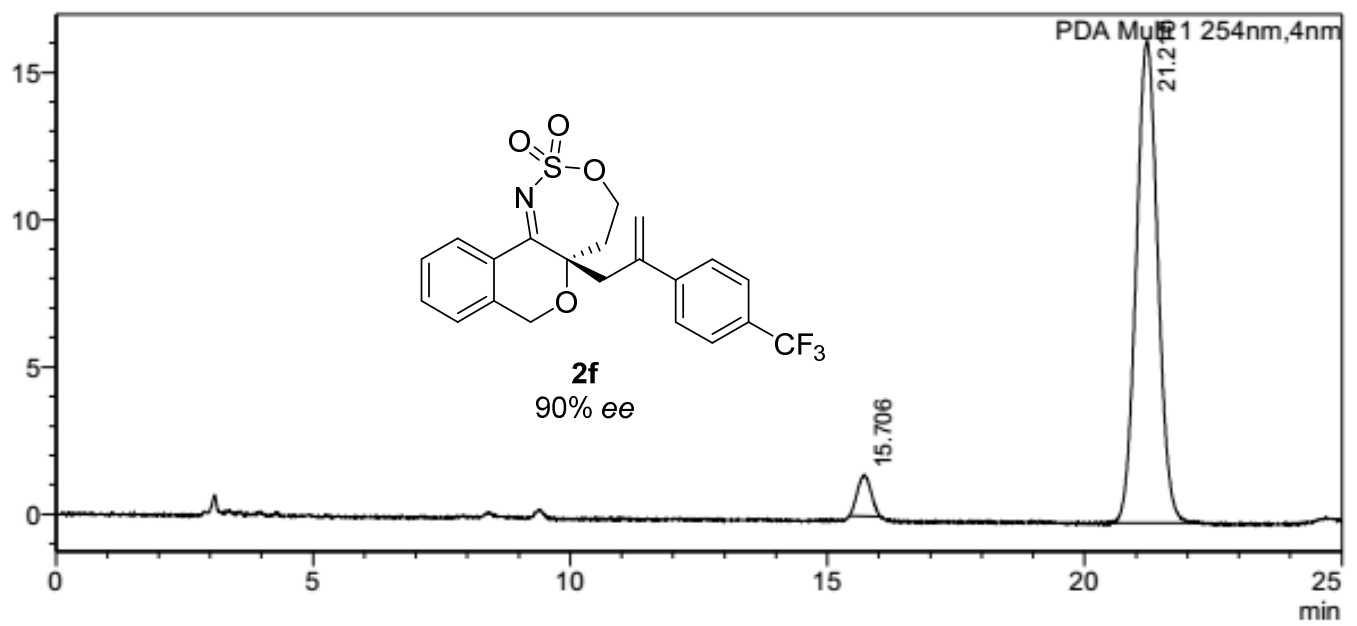

<Peak Table>

PDA Ch1 254nm

\begin{tabular}{|r|r|r|r|r|}
\hline Peak\# & Ret. Time & \multicolumn{1}{|c|}{ Area } & \multicolumn{1}{c|}{ Height } & \multicolumn{1}{c|}{ Area\% } \\
\hline 1 & 15.706 & 25750 & 1389 & 5.143 \\
\hline 2 & 21.210 & 474885 & 16332 & 94.857 \\
\hline Total & & 500635 & 17721 & 100.000 \\
\hline
\end{tabular}

Condition: hexane : 2-propanol $=90: 10$

Flow rate $=1.0 \mathrm{~mL} / \mathrm{min}, \lambda=254 \mathrm{~nm}$, Daicel Chiralcel IA

\section{$<$ Chromatogram>}

mAU

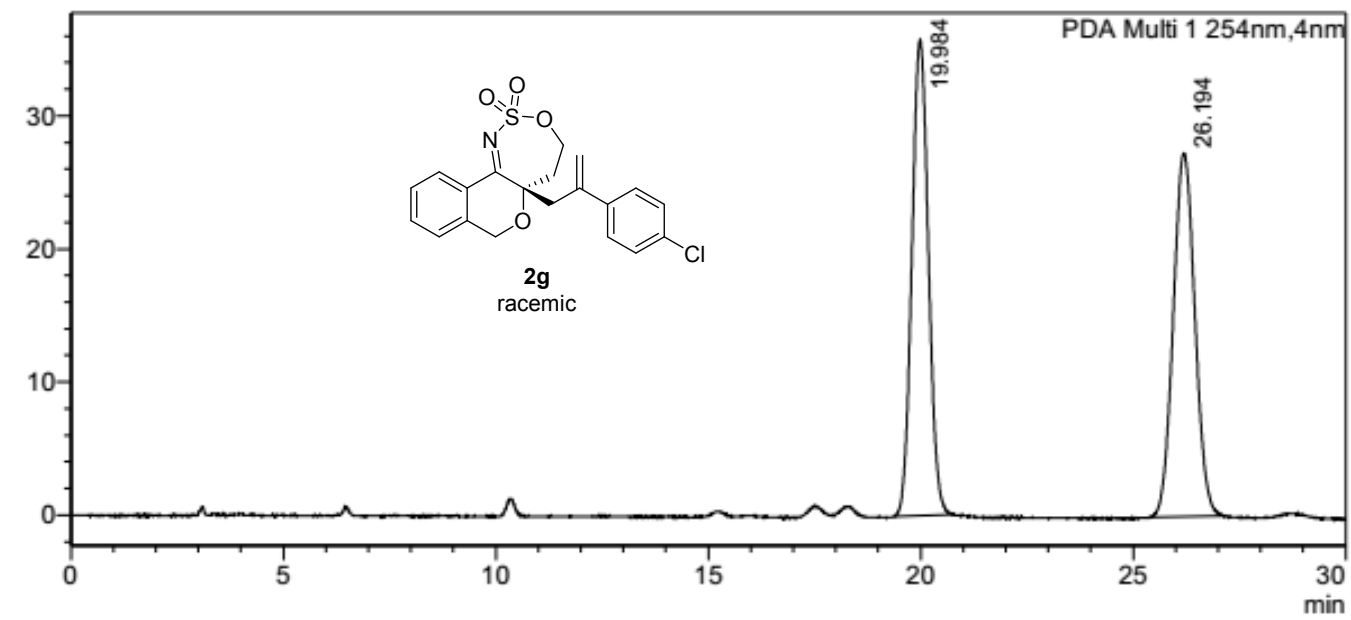

<Peak Table>

PDA Ch1 254nm

\begin{tabular}{|r|r|r|r|r|}
\hline Peak\# & Ret. Time & \multicolumn{1}{c|}{ Area } & Height & \multicolumn{1}{c|}{ Area\% } \\
\hline 1 & 19.984 & 968029 & 35764 & 49.994 \\
\hline 2 & 26.194 & 968250 & 27340 & 50.006 \\
\hline Total & & 1936279 & 63104 & 100.000 \\
\hline
\end{tabular}


$<$ Chromatogram>

mAU

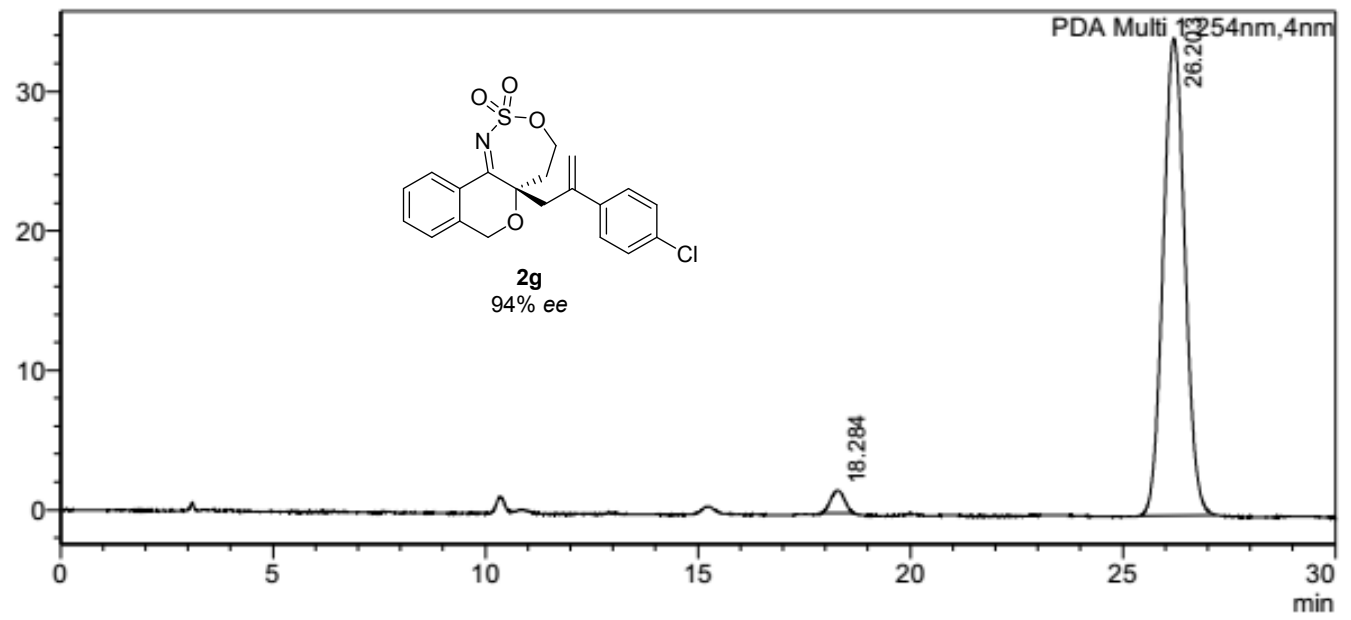

<Peak Table>

\section{PDA Ch1 254nm}

\begin{tabular}{|r|r|r|r|r|}
\hline Peak\# & Ret. Time & \multicolumn{1}{c|}{ Area } & Height & \multicolumn{1}{c|}{ Area\% } \\
\hline 1 & 18.284 & 35391 & 1621 & 2.833 \\
\hline 2 & 26.203 & 1213897 & 34211 & 97.167 \\
\hline Total & & 1249289 & 35832 & 100.000 \\
\hline
\end{tabular}

Condition: hexane $:$ 2-propanol $=90: 10$

Flow rate $=1.0 \mathrm{~mL} / \mathrm{min}, \lambda=272 \mathrm{~nm}$, Chiralpak IA-3

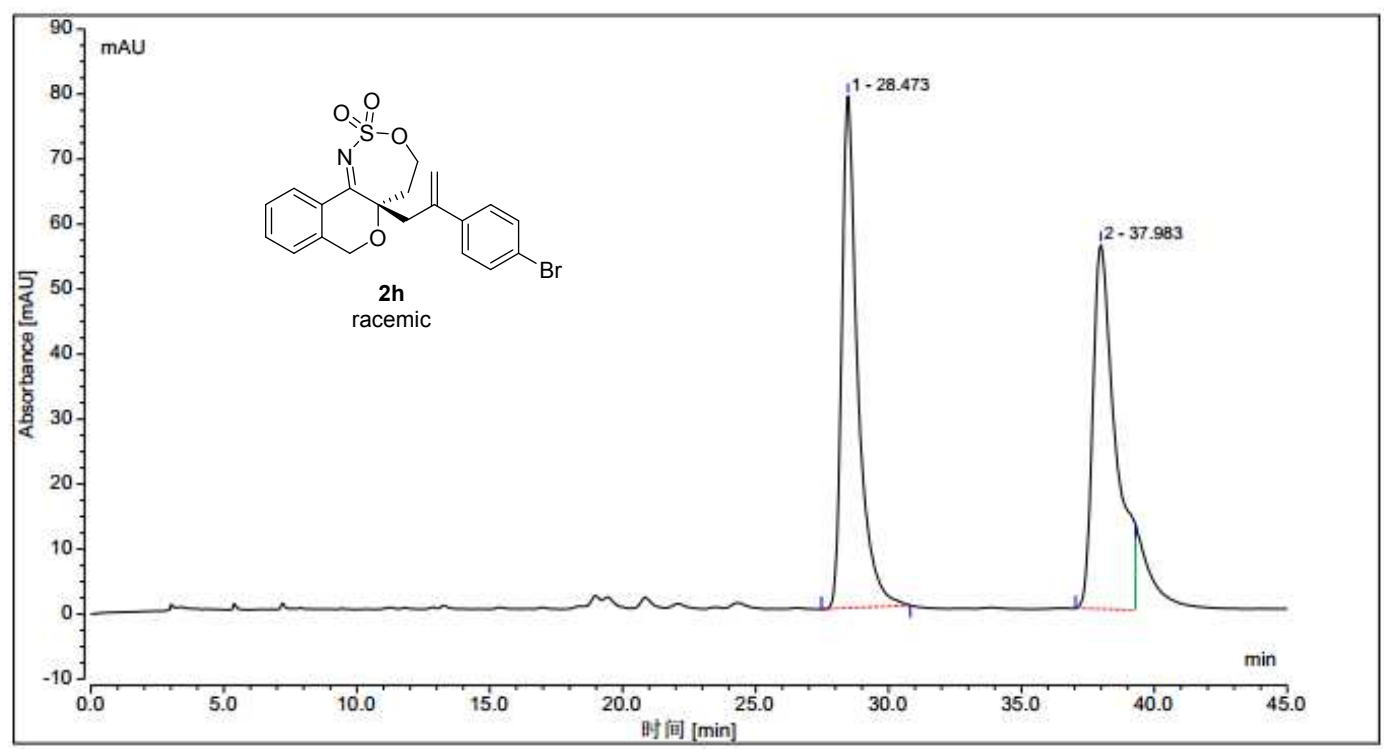

\begin{tabular}{|c|c|c|c|c|c|}
\hline Entry & $\begin{array}{c}\text { RT } \\
\text { min }\end{array}$ & $\begin{array}{c}\text { Area } \\
\text { mAU*min }\end{array}$ & $\begin{array}{c}\text { Height } \\
\text { mAU }\end{array}$ & $\begin{array}{c}\% \text { Area } \\
\%\end{array}$ & $\begin{array}{c}\% \text { Height } \\
\%\end{array}$ \\
\hline 1 & 28.473 & 55.052 & 78.766 & 50.02 & 58.40 \\
2 & 37.983 & 54.997 & 56.101 & 49.98 & 41.60 \\
\hline
\end{tabular}




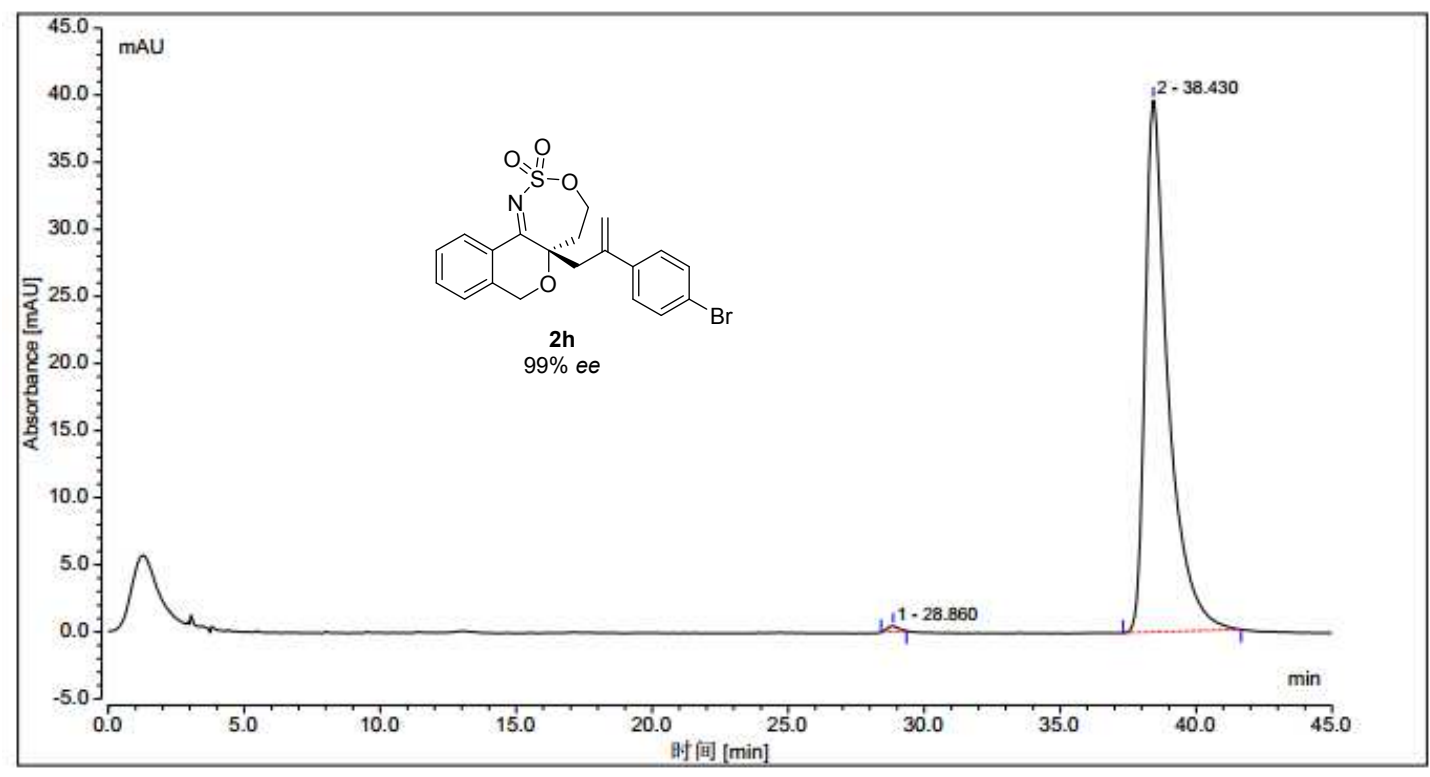

\begin{tabular}{|c|c|c|c|c|c|}
\hline Entry & $\begin{array}{c}\text { RT } \\
\text { min }\end{array}$ & $\begin{array}{c}\text { Area } \\
\text { mAU*min }\end{array}$ & $\begin{array}{c}\text { Height } \\
\text { mAU }\end{array}$ & $\begin{array}{c}\text { \% Area } \\
\%\end{array}$ & $\begin{array}{c}\% \text { Height } \\
\%\end{array}$ \\
\hline 1 & 28.860 & 0.207 & 0.425 & 0.53 & 1.06 \\
2 & 38.430 & 39.092 & 39.636 & 99.47 & 98.94
\end{tabular}

Condition: hexane $:$-propanol $=97: 3$

Flow rate $=1.0 \mathrm{~mL} / \mathrm{min}, \lambda=254 \mathrm{~nm}$, Daicel Chiralcel OD-H

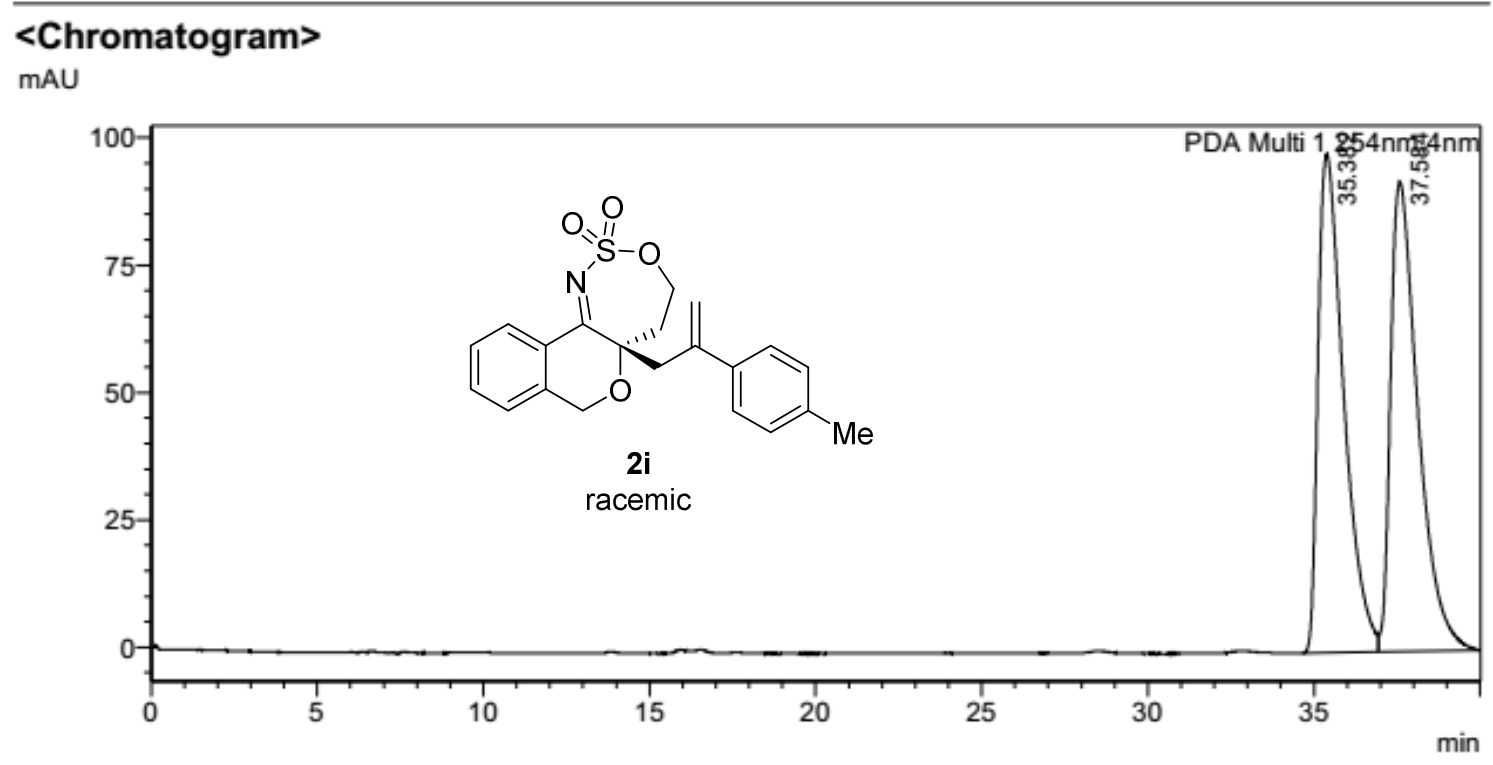

<Peak Table>

PDA Ch1 254nm

\begin{tabular}{|r|r|r|r|r|}
\hline Peak\# & Ret. Time & \multicolumn{1}{|c|}{ Area } & Height & \multicolumn{1}{c|}{ Area\% } \\
\hline 1 & 35.382 & 5256248 & 97936 & 49.770 \\
\hline 2 & 37.584 & 5304810 & 92187 & 50.230 \\
\hline Total & & 10561058 & 190123 & 100.000 \\
\hline
\end{tabular}


$<$ Chromatogram >

mAU

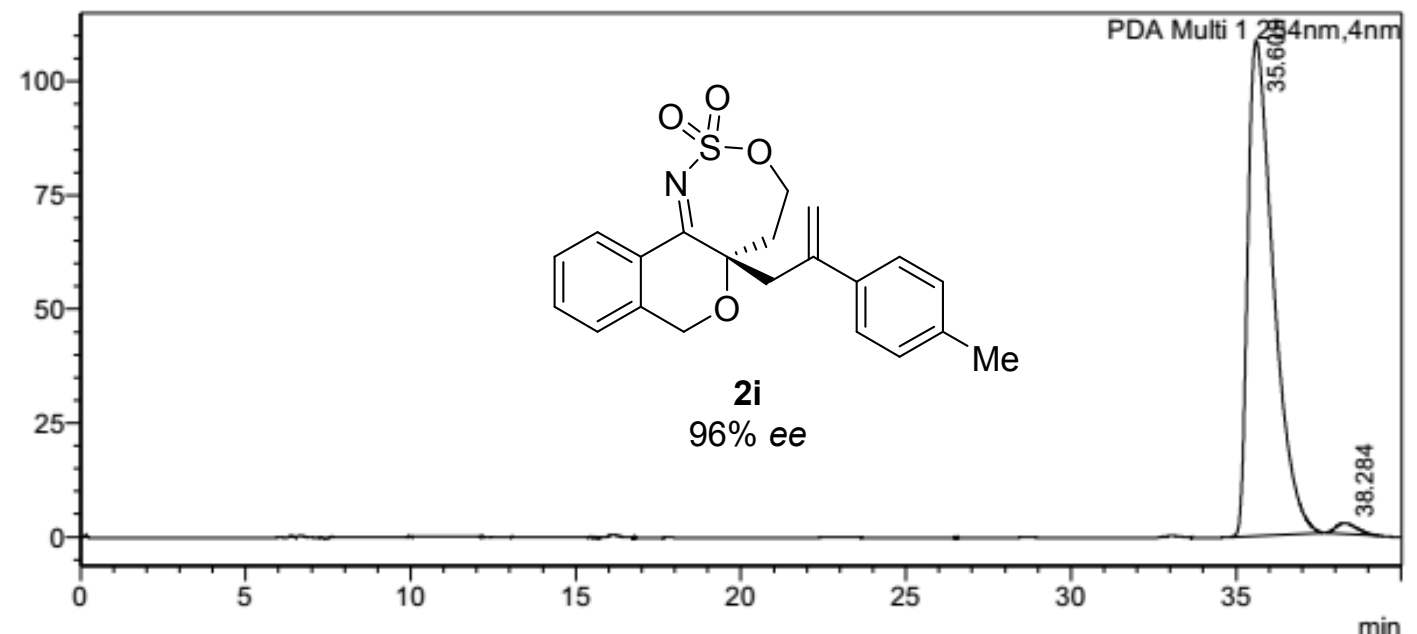

<Peak Table>

PDA Ch1 254nm

\begin{tabular}{|l|l|l|l}
\hline Peak\# Ret. Time & Area & Height & Area\% \\
\hline
\end{tabular}

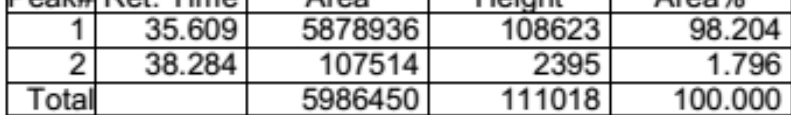

Condition: hexane $:$-propanol $=90: 10$

Flow rate $=1.0 \mathrm{~mL} / \mathrm{min}, \lambda=254 \mathrm{~nm}$, Daicel Chiralcel IA

\section{$<$ Chromatogram>}

mAU

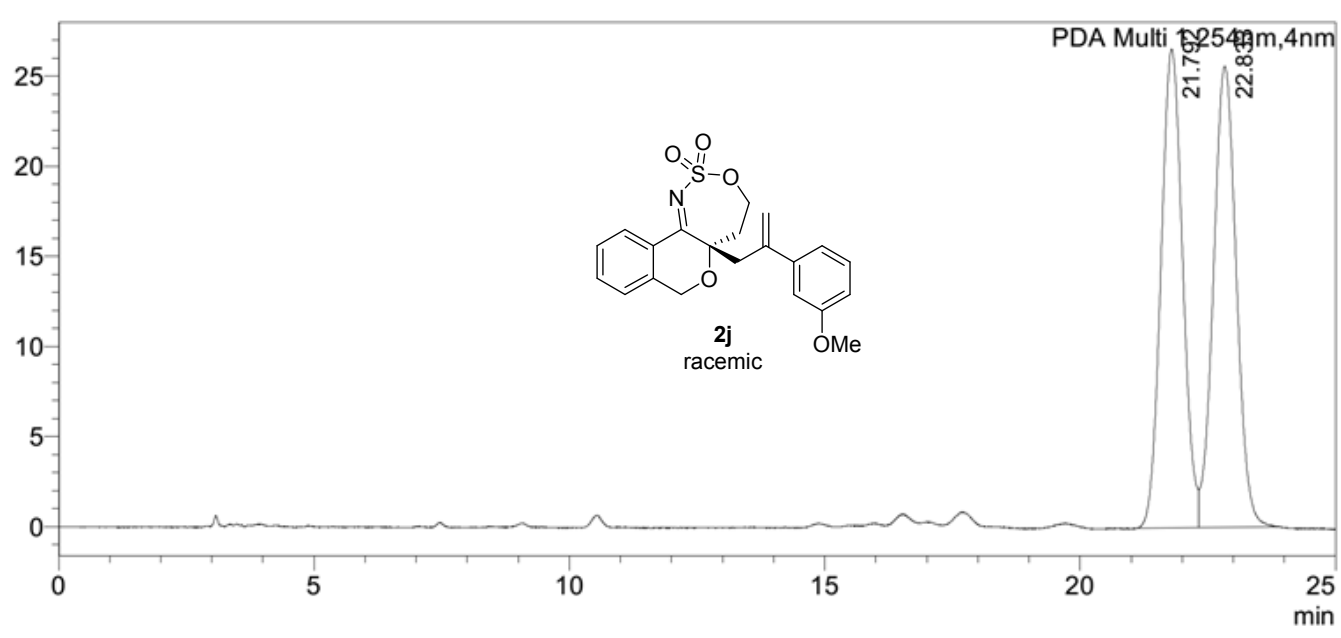

<Peak Table>

PDA Ch1 254nm

Peak\# Ret. Time

\begin{tabular}{|r|r|r|r|r|}
\hline Peak\# Ret. Time & \multicolumn{1}{|c|}{ Area } & Height & \multicolumn{1}{|c|}{ Area\% } \\
\hline 1 & 21.792 & 783367 & 26544 & 49.602 \\
\hline 2 & 22.833 & 795929 & 25587 & 50.398 \\
\hline Total & & 1579295 & 52131 & 100.000 \\
\hline
\end{tabular}


<Chromatogram>

mAU

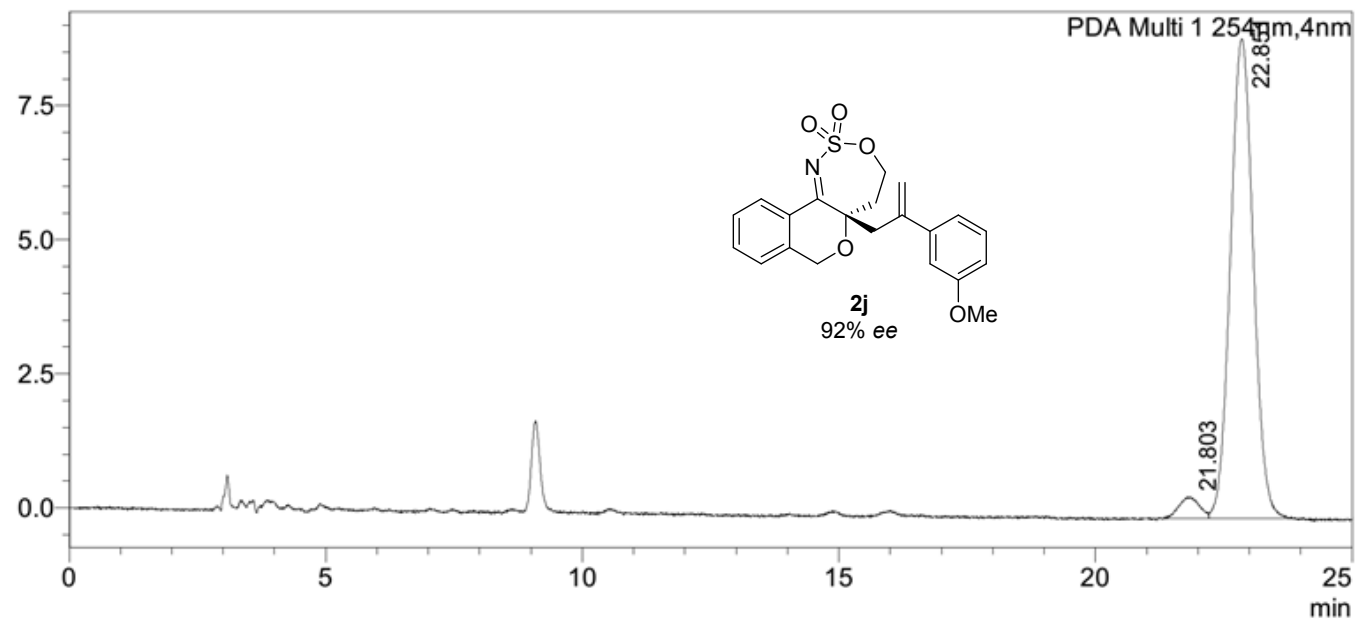

<Peak Table>

PDA Ch1 254nm

\begin{tabular}{|r|r|r|r|r|}
\hline Peak\# Ret. Time & \multicolumn{1}{|c|}{ Area } & Height & \multicolumn{1}{c|}{ Area\% } \\
\hline 1 & 21.803 & 11106 & 399 & 3.843 \\
\hline 2 & 22.851 & 277841 & 8944 & 96.157 \\
\hline Total & & 288947 & 9343 & 100.000 \\
\hline
\end{tabular}

Condition: hexane $: 2$-propanol $=90: 10$

Flow rate $=1.0 \mathrm{~mL} / \mathrm{min}, \lambda=254 \mathrm{~nm}$, Daicel Chiralcel IA

$<$ Chromatogram>

mAU

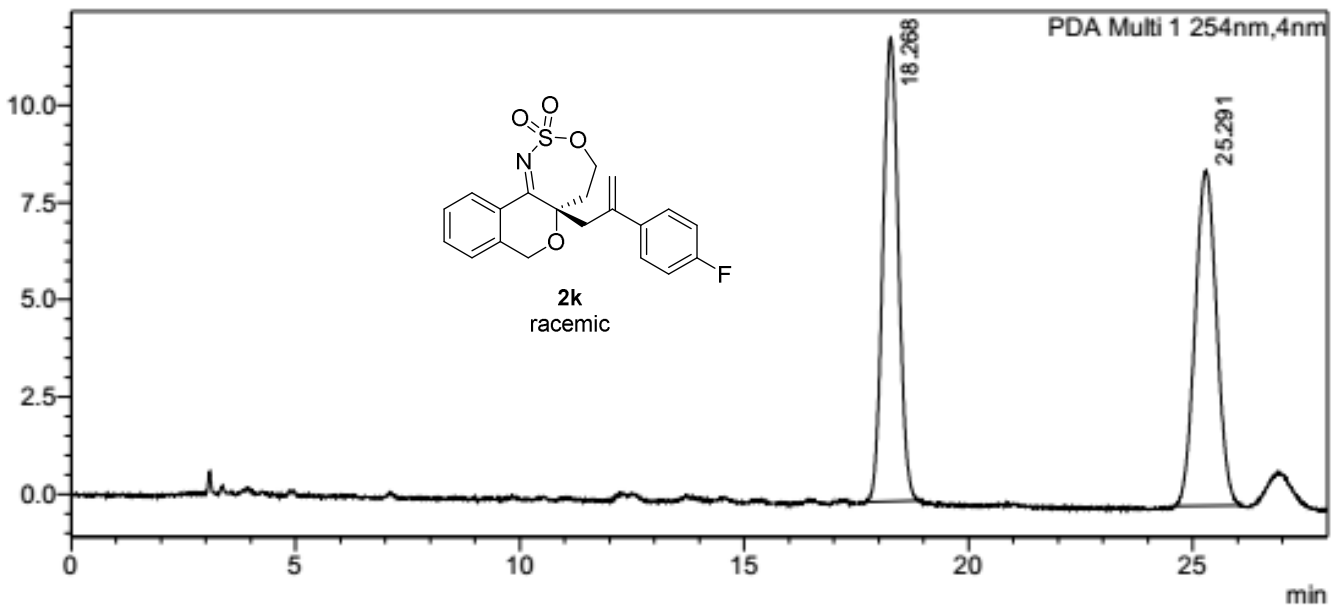

<Peak Table>

PDA Ch1 $254 \mathrm{~nm}$
\begin{tabular}{|r|r|r|r|r|}
\hline Peak\# Ret. Time & Area & \multicolumn{1}{c|}{ Height } & \multicolumn{1}{c|}{ Area $\%$} \\
\hline 1 & 18.268 & 287834 & 11909 & 50.087 \\
\hline 2 & 25.291 & 286833 & 8606 & 49.913 \\
\hline Total & & 574667 & 20515 & 100.000 \\
\hline
\end{tabular}


<Chromatogram>

mAU

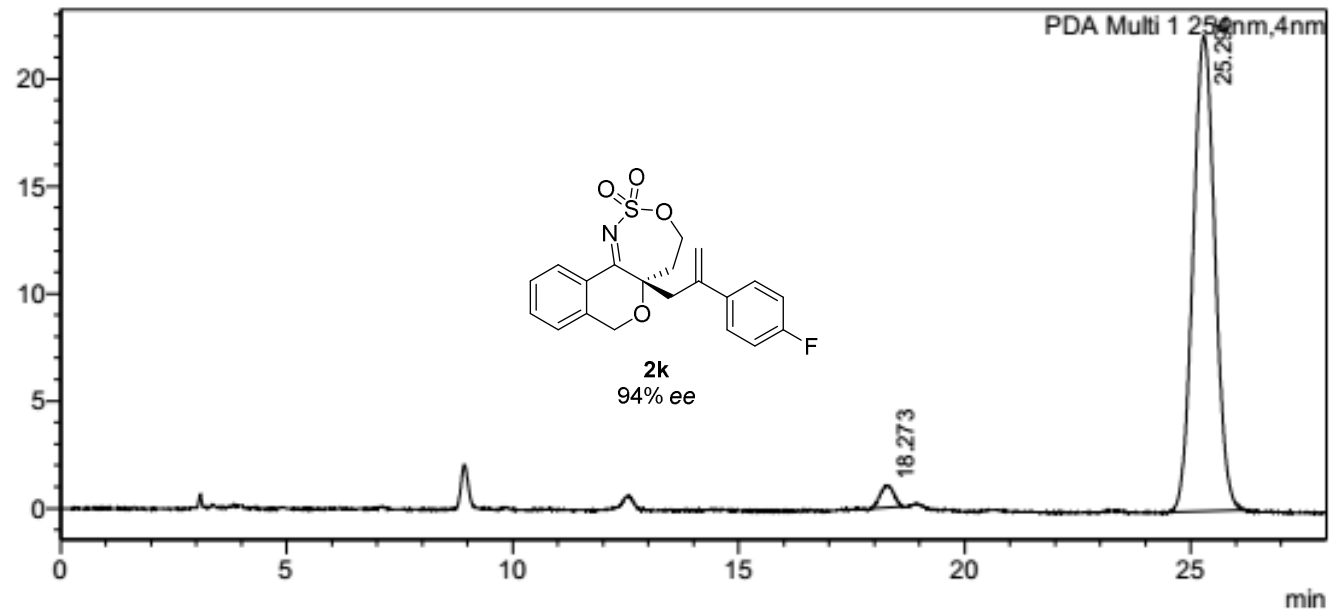

$<$ Peak Table>

PDA Ch1 254nm

\begin{tabular}{|r|r|r|r|r|}
\hline Peak\# & Ret. Time & \multicolumn{1}{c|}{ Area } & \multicolumn{1}{c|}{ Height } & \multicolumn{1}{c|}{ Area\% } \\
\hline 1 & 18.273 & 21861 & 1033 & 2.863 \\
\hline 2 & 25.295 & 741661 & 22102 & 97.137 \\
\hline Total & & 763522 & 23135 & 100.000 \\
\hline
\end{tabular}

Condition: hexane : 2-propanol $=90: 10$

Flow rate $=1.0 \mathrm{~mL} / \mathrm{min}, \lambda=254 \mathrm{~nm}$, Daicel Chiralcel IA

\section{$<$ Chromatogram>}

mAU

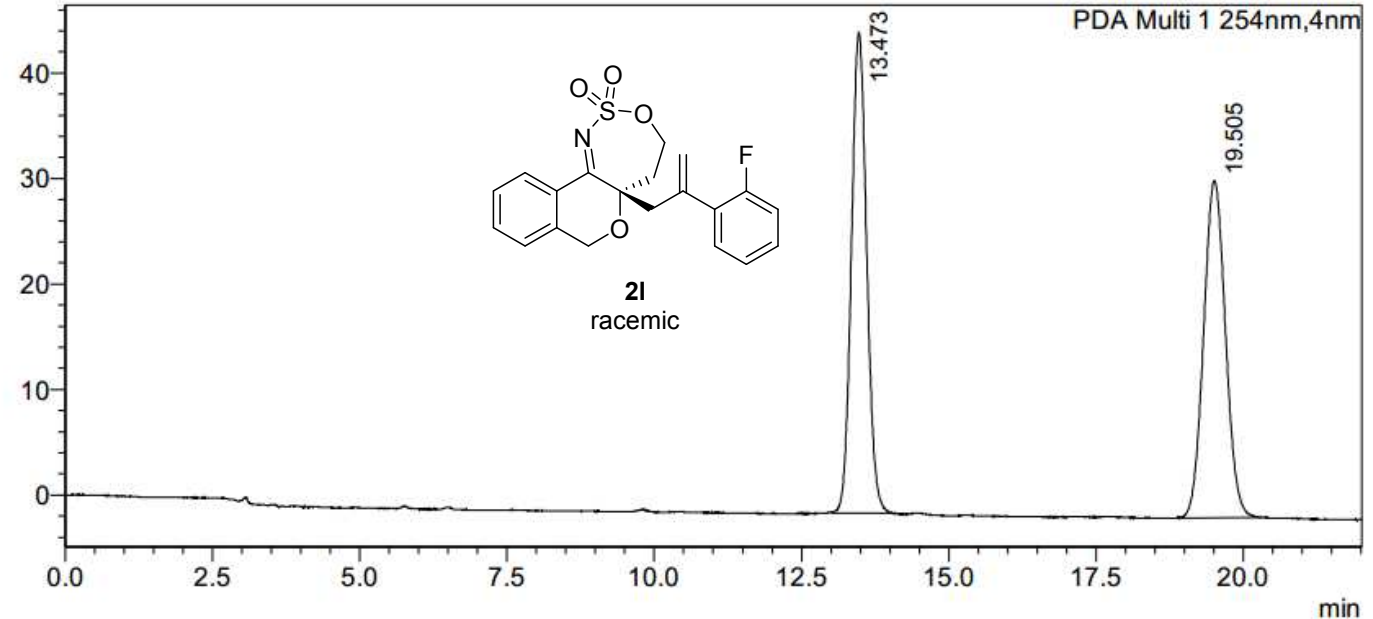

<Peak Table>

PDA Ch1 254nm

\begin{tabular}{|r|r|r|r|r|}
\hline Peak\# & Ret. Time & \multicolumn{1}{c|}{ Area } & Height & \multicolumn{1}{c|}{ Area\% } \\
\hline 1 & 13.473 & 822493 & 45549 & 49.960 \\
\hline 2 & 19.505 & 823820 & 31942 & 50.040 \\
\hline Total & & 1646313 & 77491 & 100.000 \\
\hline
\end{tabular}




\section{$<$ Chromatogram>}

mAU

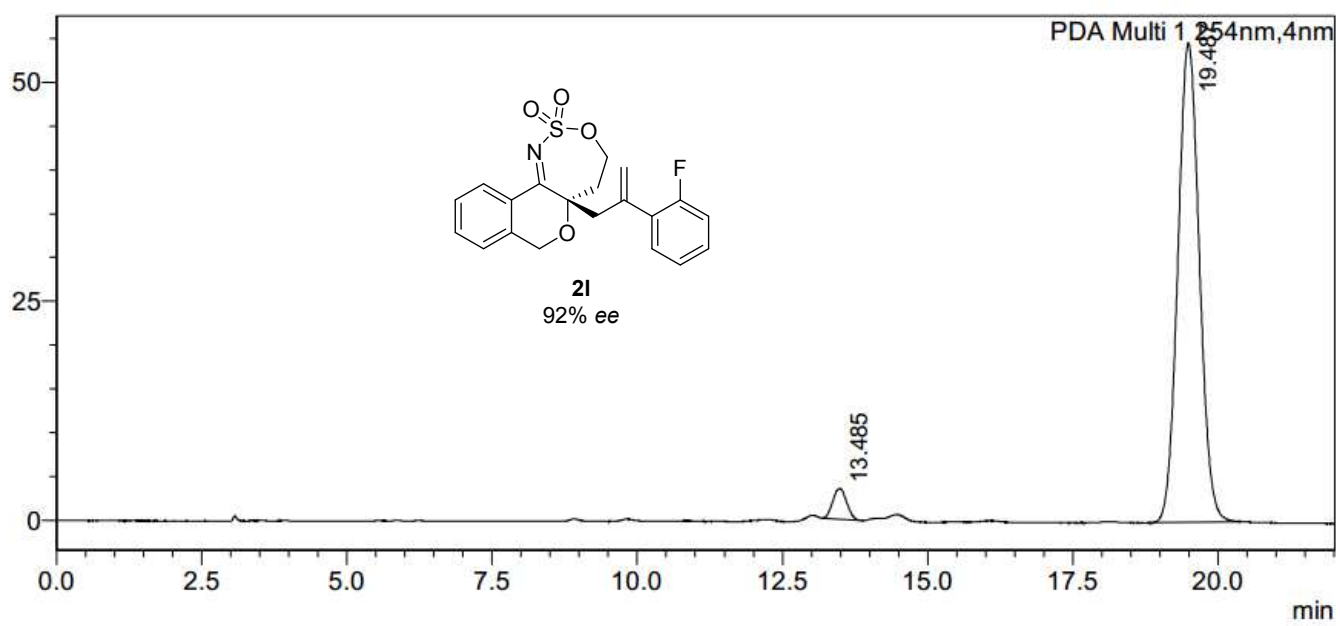

<Peak Table>

PDA Ch1 254nm

Peak\# Ret. Time

\begin{tabular}{|r|r|r|r|r|}
\hline Peak\# & Ret. Time & \multicolumn{1}{c|}{ Area } & Height & \multicolumn{1}{c|}{ Area\% } \\
\hline 1 & 13.485 & 58650 & 3541 & 3.995 \\
\hline 2 & 19.487 & 1409503 & 54722 & 96.005 \\
\hline Total & & 1468153 & 58262 & 100.000 \\
\hline
\end{tabular}

Condition: hexane $:$-propanol $=90: 10$

Flow rate $=1.0 \mathrm{~mL} / \mathrm{min}, \lambda=254 \mathrm{~nm}$, Daicel Chiralcel IA

\section{<Chromatogram>}

mAU

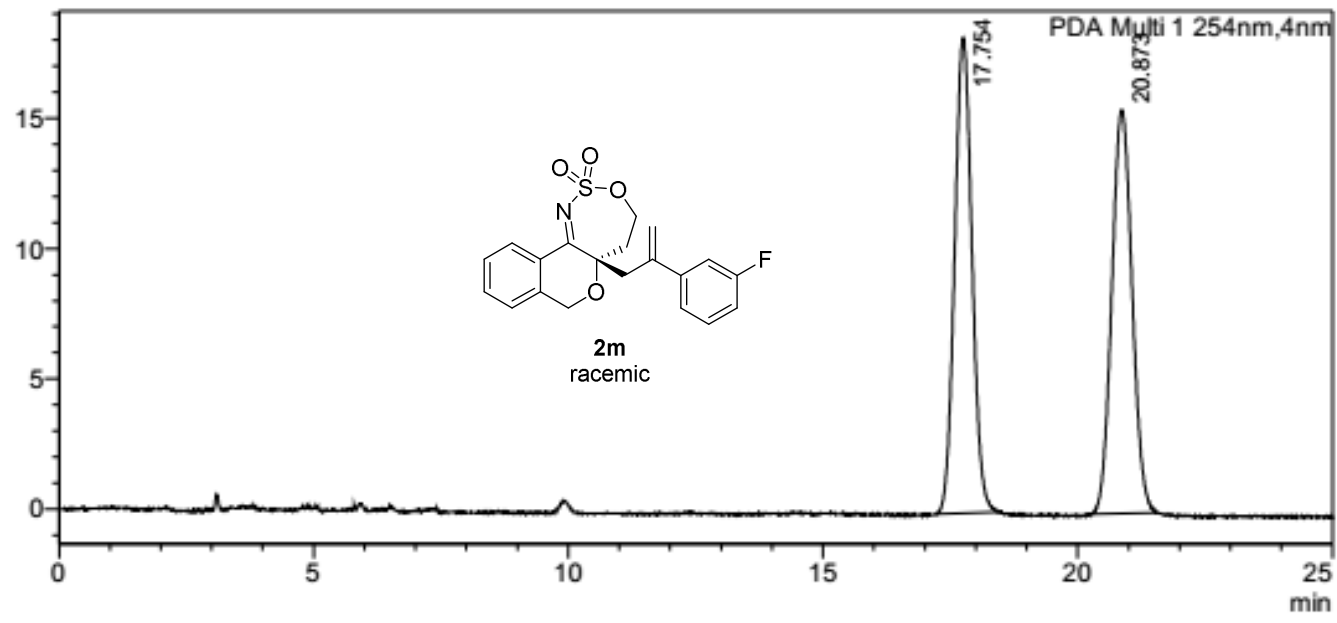

<Peak Table>

PDA Ch1 254nm

\begin{tabular}{|r|r|r|r|r|}
\hline Peak\# & Ret. Time & \multicolumn{1}{|c|}{ Area } & \multicolumn{1}{c|}{ Height } & \multicolumn{1}{|c|}{ Area\% } \\
\hline 1 & 17.754 & 433622 & 18240 & 50.297 \\
\hline 2 & 20.873 & 428496 & 15484 & 49.703 \\
\hline Total & & 862118 & 33724 & 100.000 \\
\hline
\end{tabular}




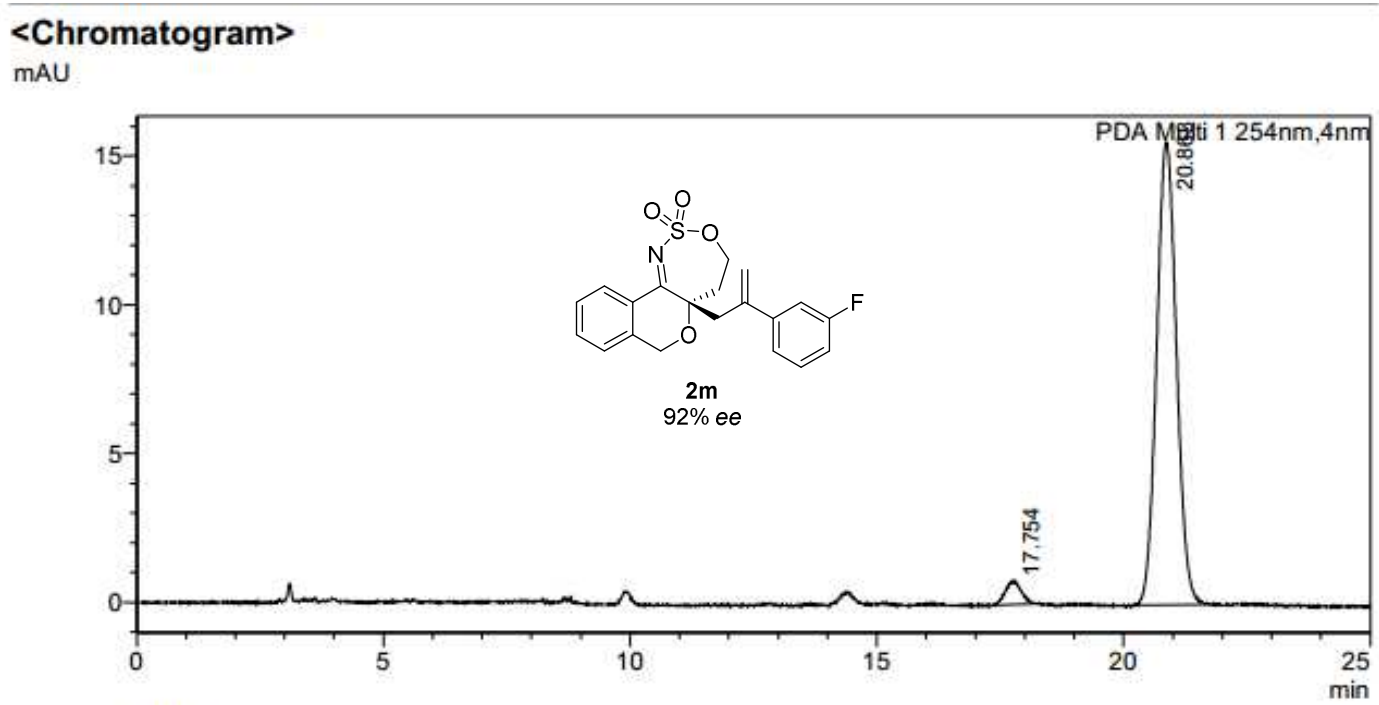

<Peak Table>

PDA Ch1 254nm

\begin{tabular}{|c|c|c|c|c|}
\hline Peak\# & Ret. Time & Area & Height & Area $\%$ \\
\hline 1 & 17.754 & 17747 & 784 & 3.929 \\
\hline 2 & 20.868 & 433992 & 15547 & 96.071 \\
\hline Total & & 451738 & 16331 & 100.000 \\
\hline
\end{tabular}

Condition: hexane $: 2$-propanol $=90: 1$

Flow rate $=1.0 \mathrm{~mL} / \mathrm{min}, \lambda=254 \mathrm{~nm}$, Daicel Chiralcel IA

\section{$<$ Chromatogram >} $\mathrm{mAU}$

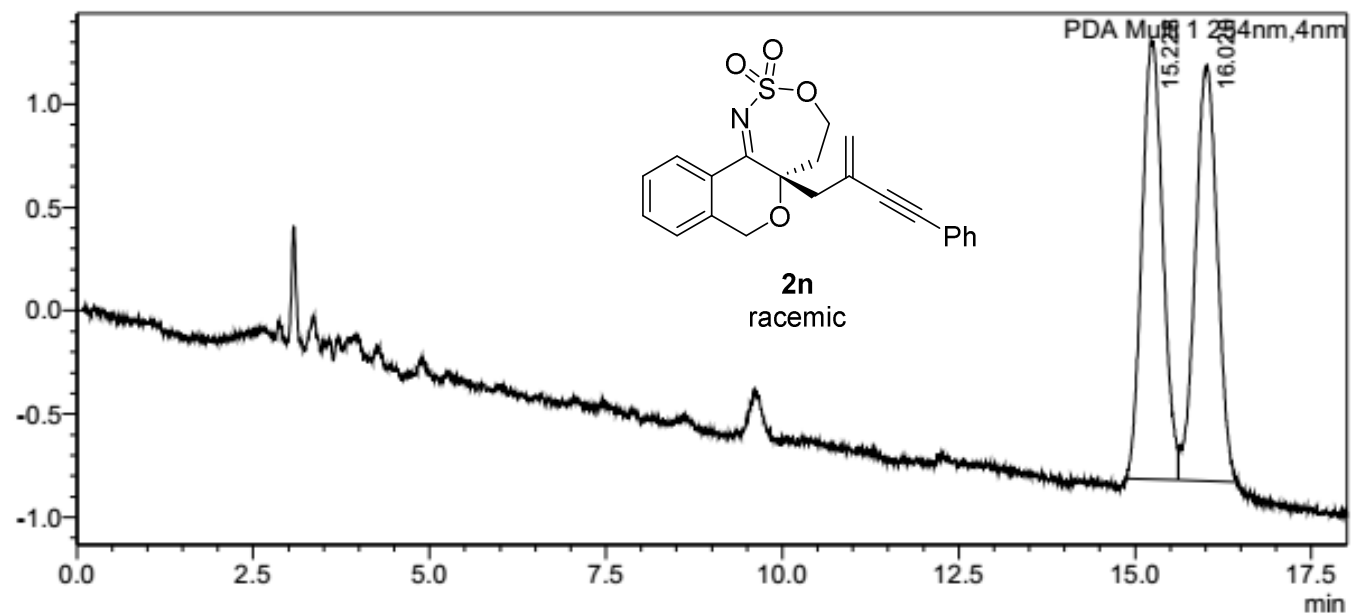

<Peak Table>

PDA Ch1 254nm

\begin{tabular}{|c|c|c|c|c|}
\hline Peak\#\# & Ret. Time & Area & Height & Area $\%$ \\
\hline 1 & 15.228 & 43672 & 2125 & 50.003 \\
\hline 2 & 16.020 & 43666 & 2014 & 49.997 \\
\hline Total & & 87338 & 4139 & 100.000 \\
\hline
\end{tabular}


$<$ Chromatogram>

mAU

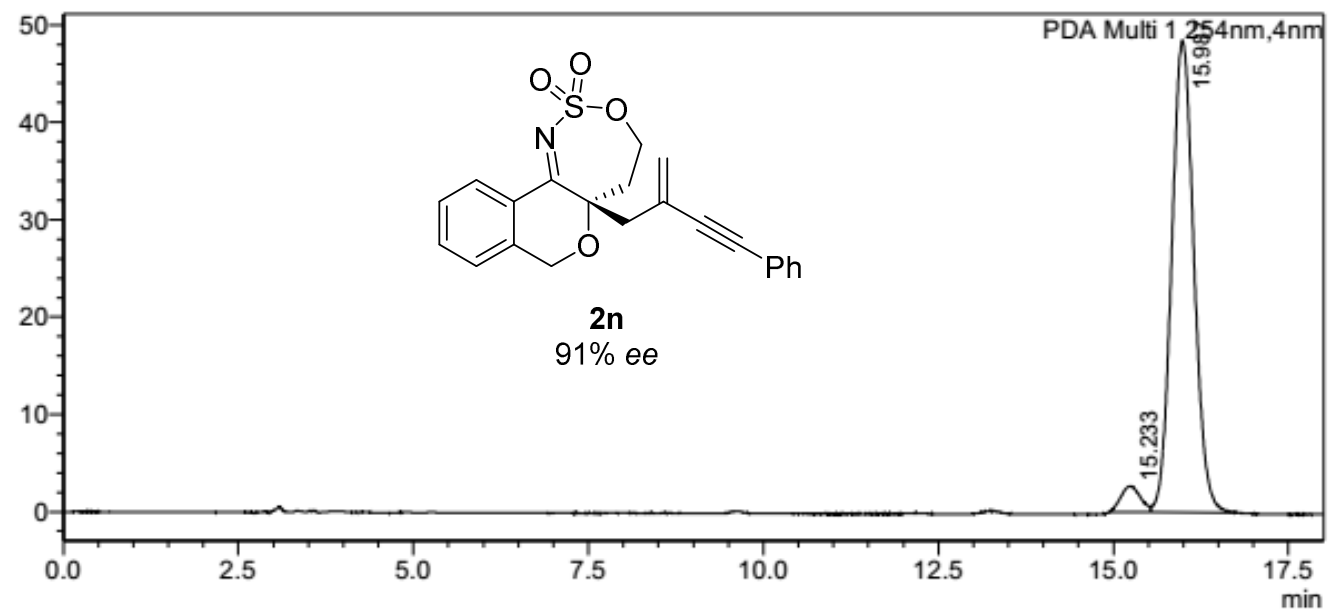

<Peak Table>

PDA Ch1 254nm

\begin{tabular}{|r|r|r|r|r|}
\hline Peak\# & Ret. Time & \multicolumn{1}{|c|}{ Area } & Height & \multicolumn{1}{c|}{ Area\% } \\
\hline 1 & 15.233 & 52767 & 2677 & 4.691 \\
\hline 2 & 15.987 & 1072134 & 48462 & 95.309 \\
\hline Total & & 1124901 & 51140 & 100.000 \\
\hline
\end{tabular}

Condition: hexane : 2-propanol $=90: 10$

Flow rate $=1.0 \mathrm{~mL} / \mathrm{min}, \lambda=254 \mathrm{~nm}$, Daicel Chiralcel IA

$<$ Chromatogram>

mAU

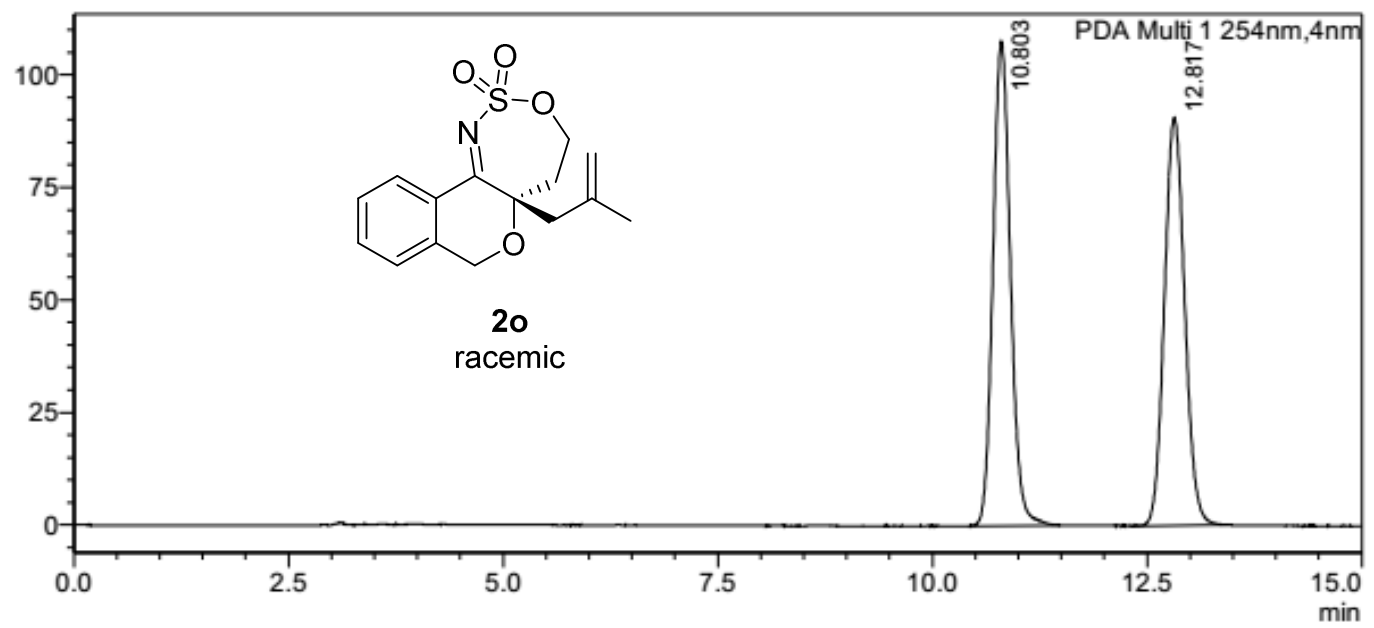

<Peak Table>

PDA Ch1 254nm

\begin{tabular}{|r|r|r|r|r|}
\hline Peak\# & Ret. Time & \multicolumn{1}{c|}{ Area } & \multicolumn{1}{c|}{ Height } & \multicolumn{1}{c|}{ Area\% } \\
\hline 1 & 10.803 & 1521671 & 107576 & 50.484 \\
\hline 2 & 12.817 & 1492470 & 90710 & 49.516 \\
\hline Total & & 3014141 & 198286 & 100.000 \\
\hline
\end{tabular}


<Chromatogram>

mAU

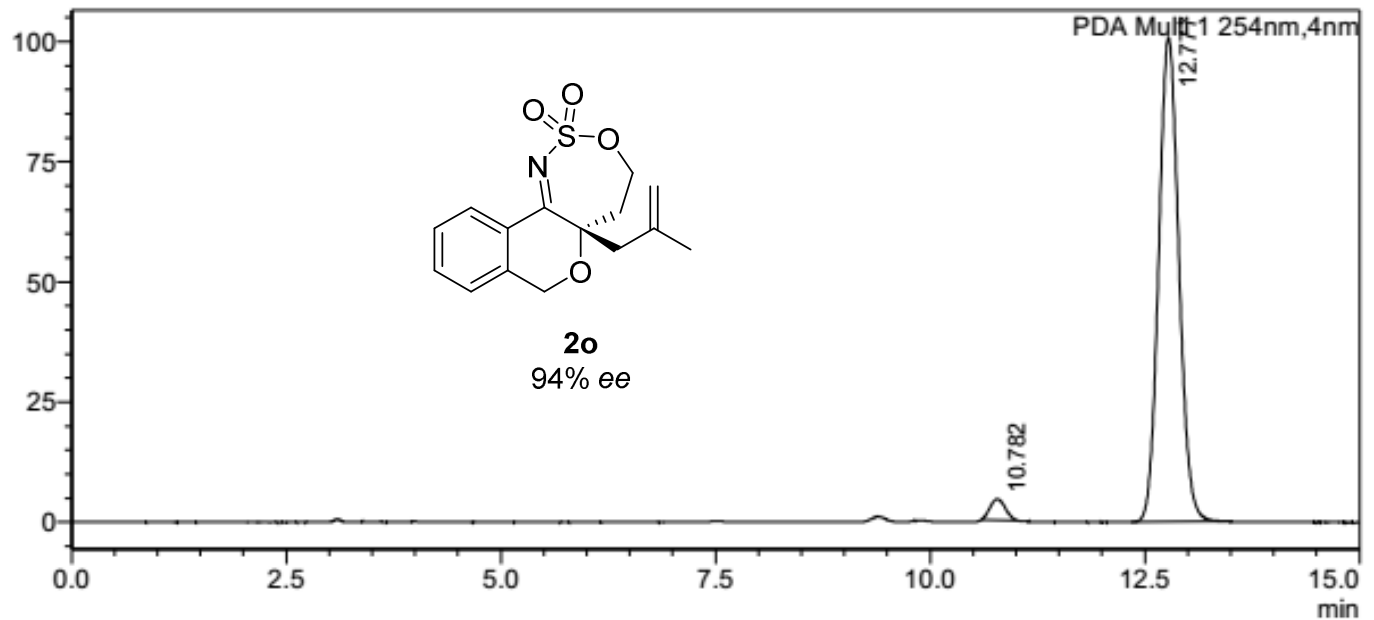

<Peak Table>

PDA Ch1 254nm

\begin{tabular}{|r|r|r|r|r|}
\hline Peak\# & Ret. Time & \multicolumn{1}{c|}{ Area } & \multicolumn{1}{c|}{ Height } & \multicolumn{1}{c|}{ Area\% } \\
\hline 1 & 10.782 & 53217 & 4326 & 3.124 \\
\hline 2 & 12.777 & 1650023 & 100785 & 96.876 \\
\hline Total & & 1703240 & 105111 & 100.000 \\
\hline
\end{tabular}

Condition: hexane $:$-propanol $=90: 10$

Flow rate $=1.0 \mathrm{~mL} / \mathrm{min}, \lambda=272 \mathrm{~nm}$, Chiralpak IA-3

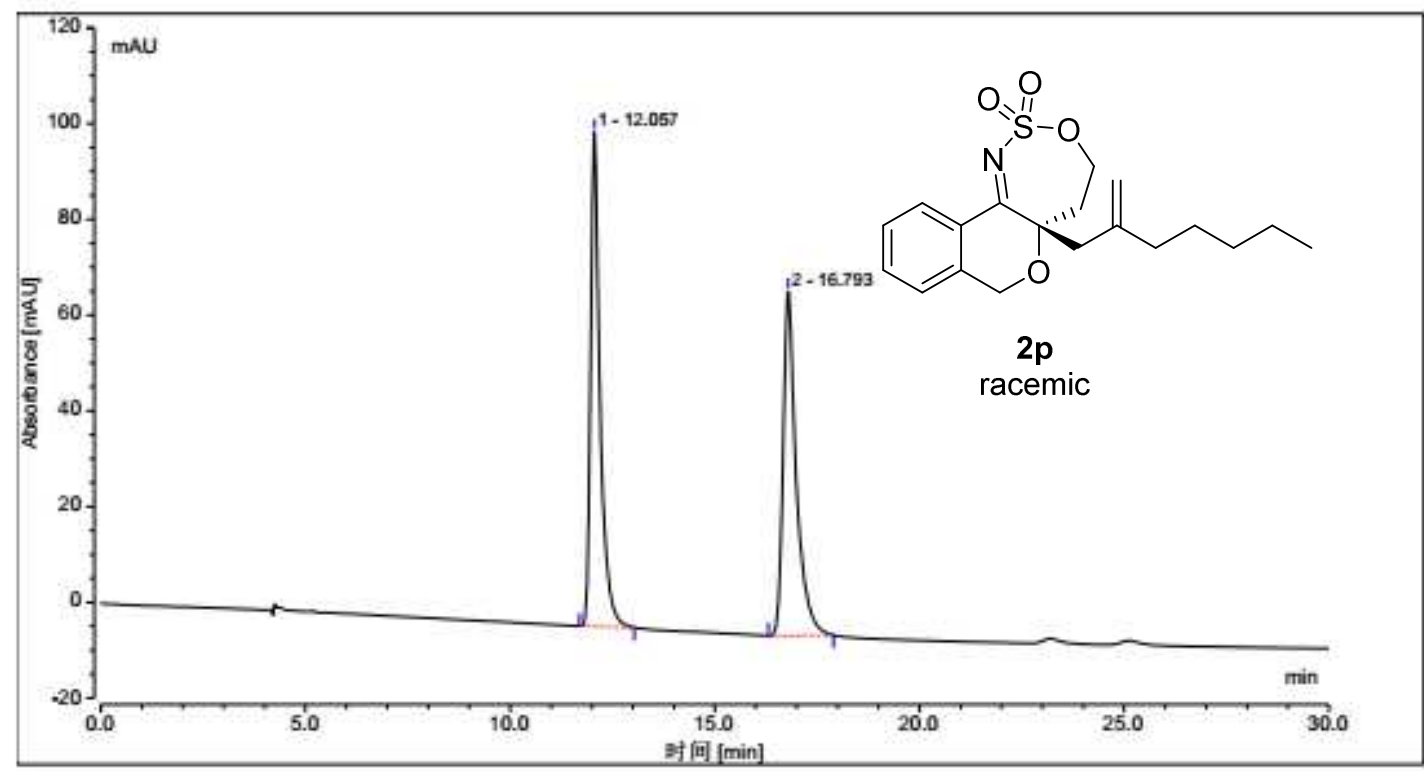

\begin{tabular}{|c|c|c|c|c|c}
\hline Entry & $\begin{array}{c}\text { RT } \\
\text { min }\end{array}$ & $\begin{array}{c}\text { Avea } \\
\text { mAƯmin }\end{array}$ & $\begin{array}{c}\text { Height } \\
\text { mAU }\end{array}$ & $\begin{array}{c}\text { \% Area } \\
\%\end{array}$ & $\begin{array}{c}\text { \% Height } \\
\%\end{array}$ \\
\hline 1 & 12.057 & 27.395 & 103.225 & 50.62 & 58.94 \\
2 & 16.793 & 26.725 & 71.906 & 49.38 & 41.06
\end{tabular}




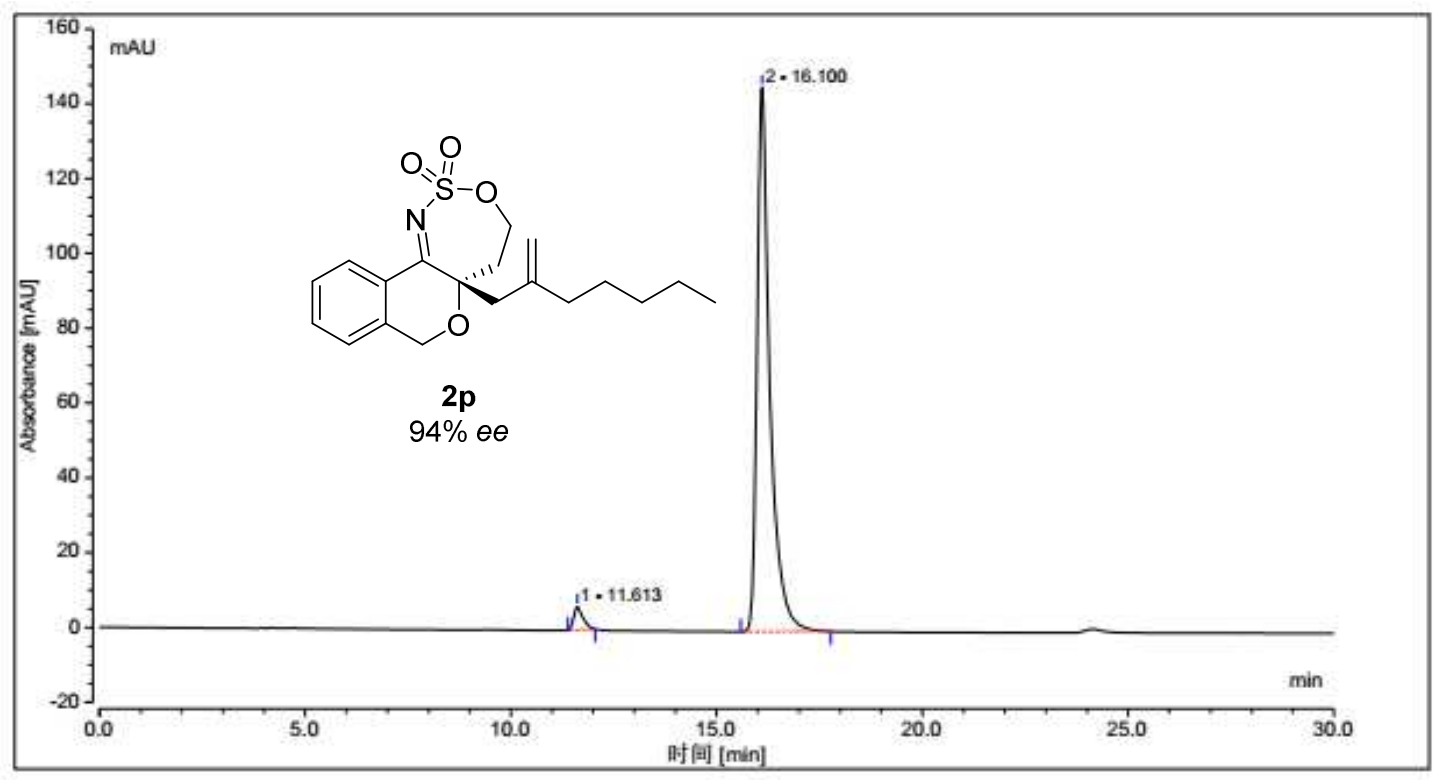

\begin{tabular}{|c|c|c|c|c|c|}
\hline Entry & $\begin{array}{c}\text { RT } \\
\text { min }\end{array}$ & $\begin{array}{c}\text { Area } \\
\text { mAU*min }\end{array}$ & $\begin{array}{c}\text { Height } \\
\text { mAU }\end{array}$ & $\begin{array}{c}\% \text { Area } \\
\%\end{array}$ & $\begin{array}{c}\% \text { Height } \\
\%\end{array}$ \\
\hline 1 & 11.613 & 1.594 & 6.156 & 2.96 & 4.07 \\
2 & 16.100 & 52.208 & 145.266 & 97.04 & 95.93
\end{tabular}

Condition: hexane $: 2$-propanol $=90: 10$

Flow rate $=1.0 \mathrm{~mL} / \mathrm{min}, \lambda=272 \mathrm{~nm}$, Chiralpak IA-3

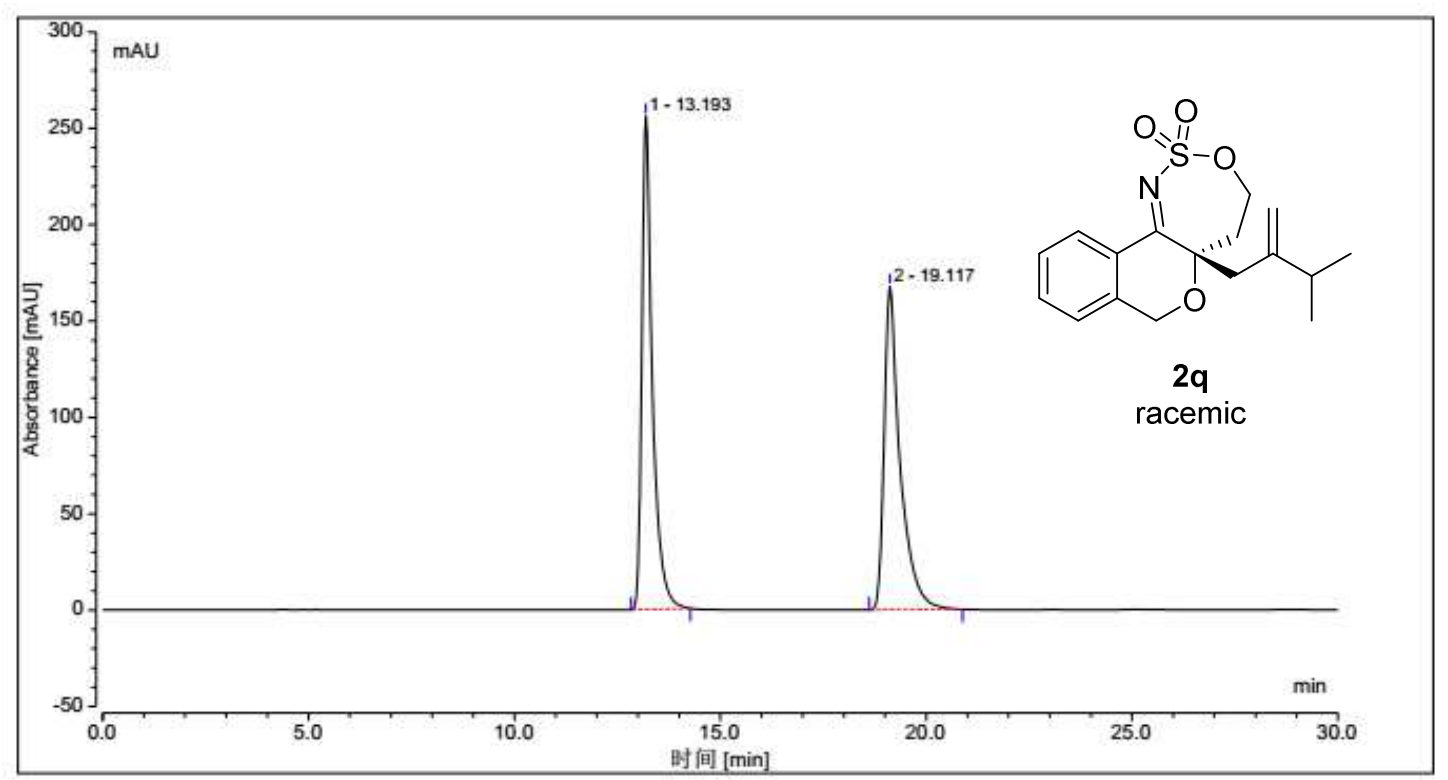

\begin{tabular}{|c|c|c|c|c|c|}
\hline Entry & $\begin{array}{c}\text { RT } \\
\mathrm{min}\end{array}$ & $\begin{array}{c}\text { Area } \\
\text { mAU*min }\end{array}$ & $\begin{array}{c}\text { Height } \\
\text { mAU }\end{array}$ & $\begin{array}{c}\text { \% Area } \\
\%\end{array}$ & $\begin{array}{c}\% \text { Height } \\
\%\end{array}$ \\
\hline 1 & 13.193 & 75.528 & 255.898 & 50.01 & 60.44 \\
2 & 19.117 & 75.501 & 167.488 & 49.99 & 39.56
\end{tabular}




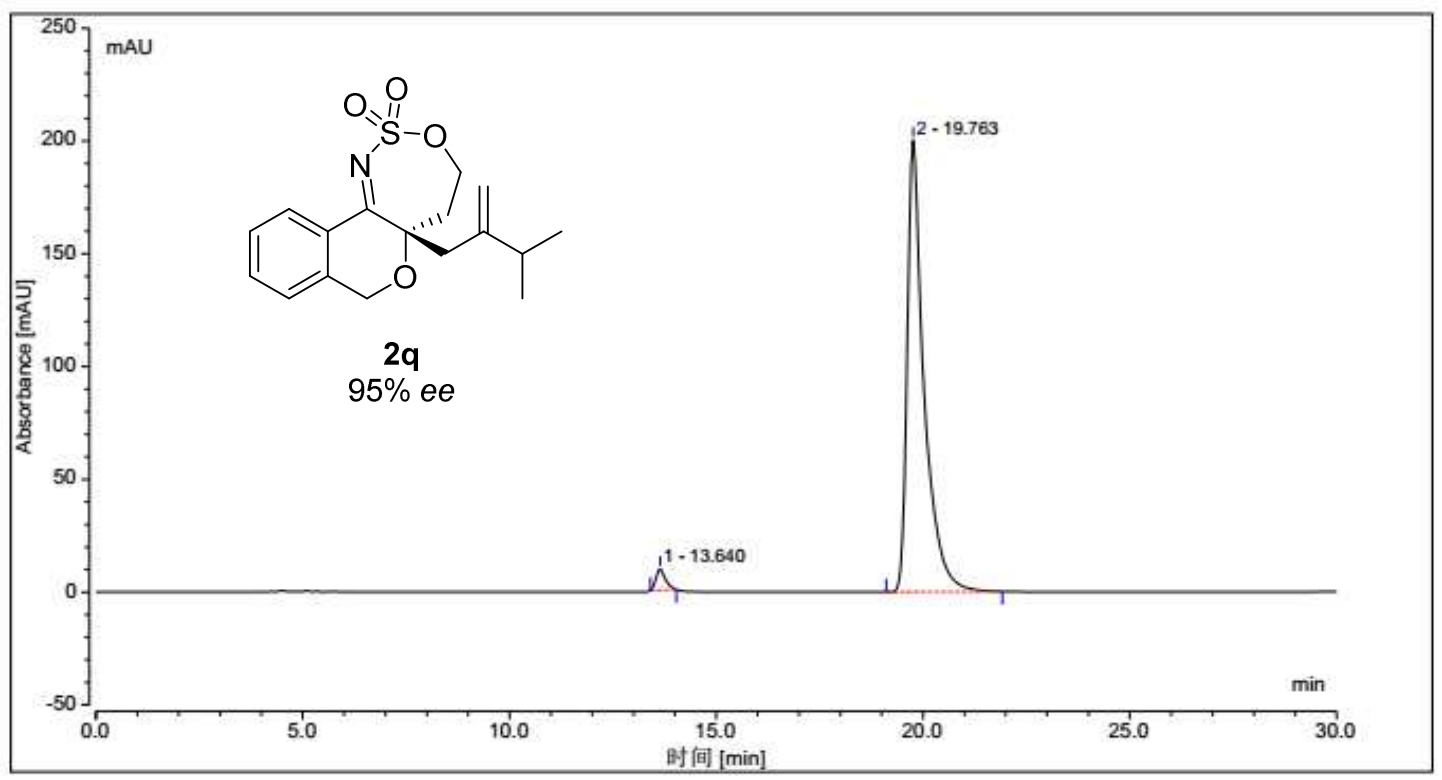

\begin{tabular}{|c|c|c|c|c|c|}
\hline Entry & $\begin{array}{c}\text { RT } \\
\text { min }\end{array}$ & $\begin{array}{c}\text { Area } \\
\text { mAU*min }\end{array}$ & $\begin{array}{c}\text { Height } \\
\text { mAU }\end{array}$ & $\begin{array}{c}\text { \% Area } \\
\%\end{array}$ & $\begin{array}{c}\% \text { Height } \\
\%\end{array}$ \\
\hline 1 & 13.640 & 2.445 & 9.361 & 2.54 & 4.47 \\
2 & 19.763 & 93.861 & 200.270 & 97.46 & 95.53
\end{tabular}

Condition: hexane $:$-propanol $=90: 10$

Flow rate $=1.0 \mathrm{~mL} / \mathrm{min}, \lambda=254 \mathrm{~nm}$, Daicel Chiralcel IA

\section{$<$ Chromatogram>}

mAU

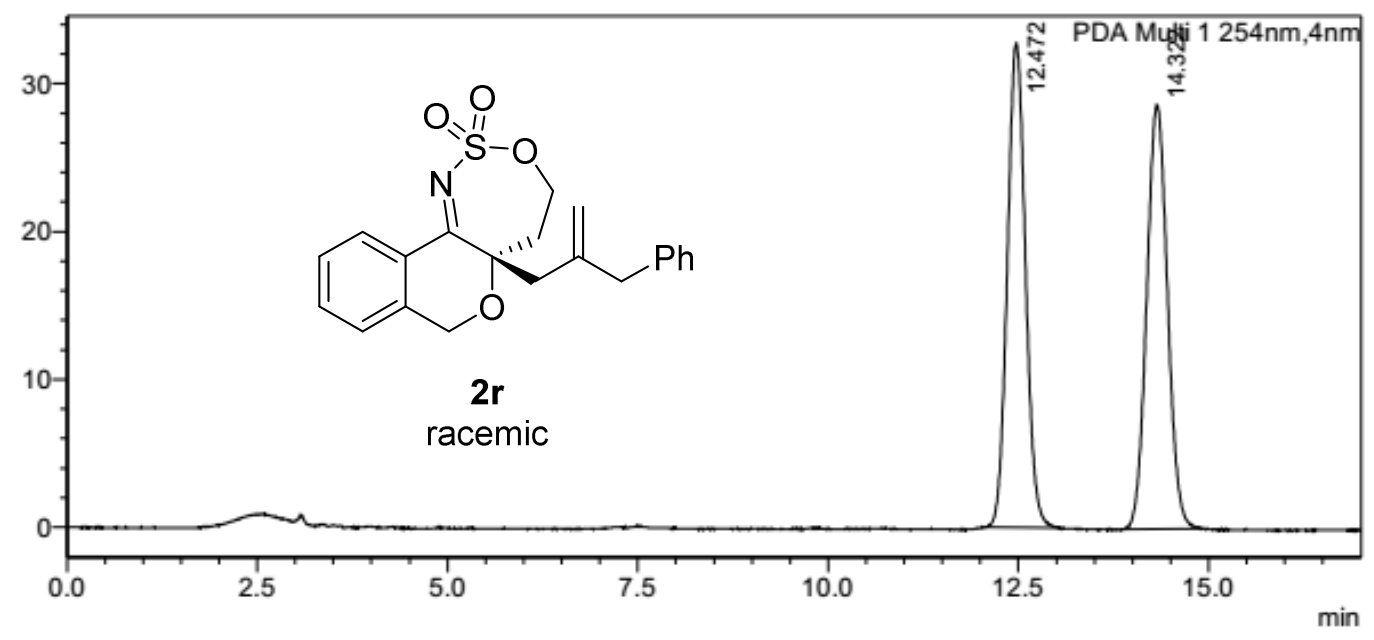

<Peak Table>

PDA Ch1 254nm

\begin{tabular}{|c|c|c|c|c|}
\hline Peak\# & et. Time & Area & Height & Area $\%$ \\
\hline 1 & 12.472 & 541626 & 32716 & 49.825 \\
\hline 2 & 14.322 & 545440 & 28704 & 50.175 \\
\hline Total & & 1087066 & 61420 & 100.000 \\
\hline
\end{tabular}




\section{<Chromatogram>}

mAU

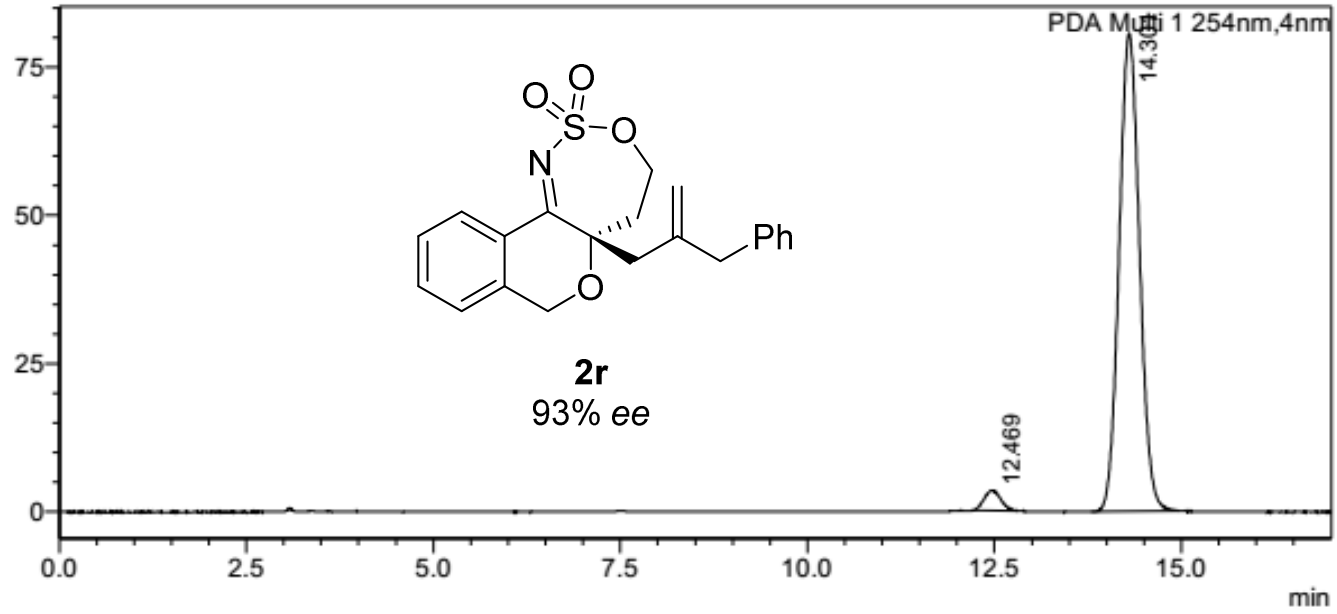

<Peak Table>

PDA Ch1 254nm

\begin{tabular}{|c|c|c|c|c|}
\hline Peak\# & et. Time & Area & Height & Area\% \\
\hline 1 & 12.469 & 52483 & 3397 & 3.319 \\
\hline 2 & 14.301 & 1528766 & 80574 & 96.681 \\
\hline Total & & 1581249 & 83971 & 100.000 \\
\hline
\end{tabular}

Condition: hexane $:$-propanol $=90: 10$

Flow rate $=1.0 \mathrm{~mL} / \mathrm{min}, \lambda=254 \mathrm{~nm}$, Daicel Chiralcel IA.

\section{$<$ Chromatogram $>$}

mAU

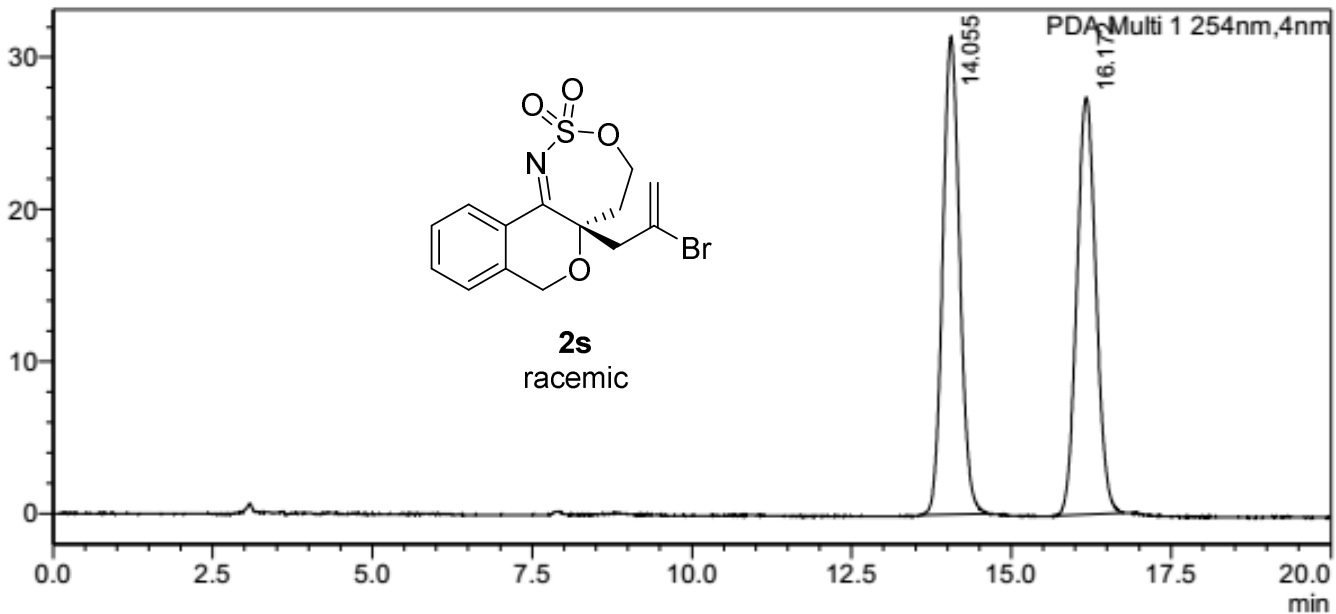

<Peak Table>

PDA Ch1 254nm

\begin{tabular}{|l|l|l|l}
\hline Peak\# Ret. Time & Area & Height & Area\%
\end{tabular}

\begin{tabular}{|r|r|r|r|r|}
\hline 1 & 14.055 & 580241 & 31432 & \multicolumn{1}{c|}{ Area\% } \\
\hline 2 & 16.172 & 572028 & 27425 & 49.644 \\
\hline Total & & 1152269 & 58857 & 100.000 \\
\hline
\end{tabular}


<Chromatogram>

mAU

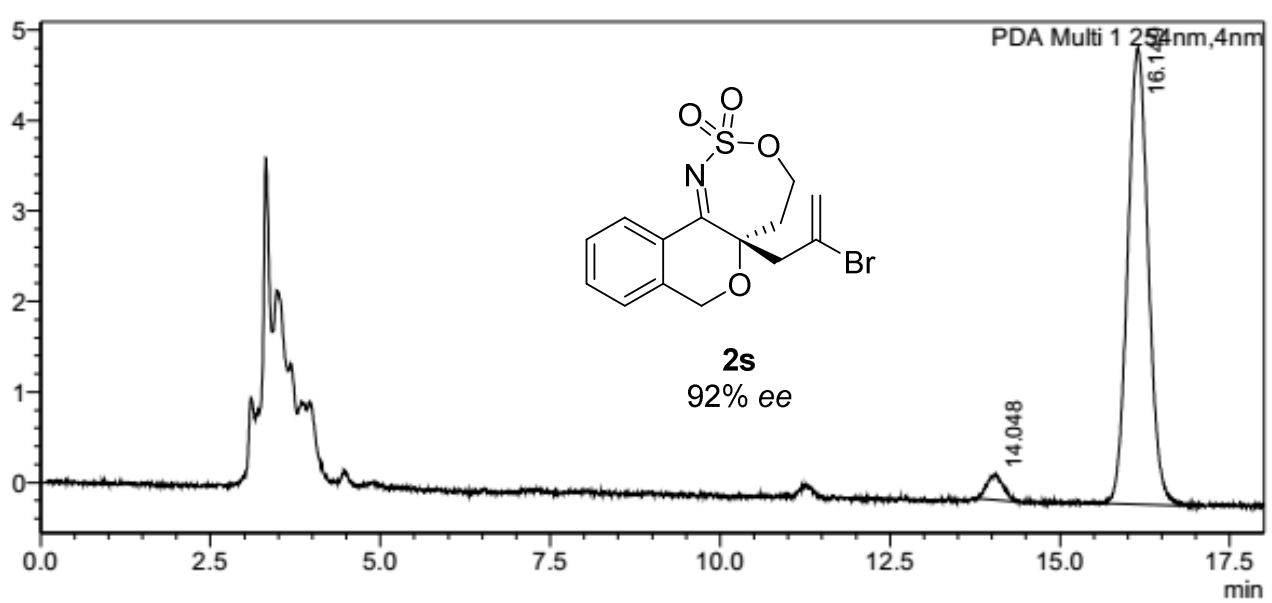

<Peak Table>

PDA Ch1 254nm

\begin{tabular}{|l|l|l|l}
\hline Peak\# Ret. Time & Area & Height & Area\% \\
\hline
\end{tabular}

\begin{tabular}{|r|r|r|r|r|}
\hline 1 & 14.048 & 4574 & 282 & 4.112 \\
\hline 2 & 16.149 & 106674 & 5047 & 95.888 \\
\hline Total & & 111249 & 5329 & 100.000 \\
\hline
\end{tabular}

$<$ Chromatogram>

mAU

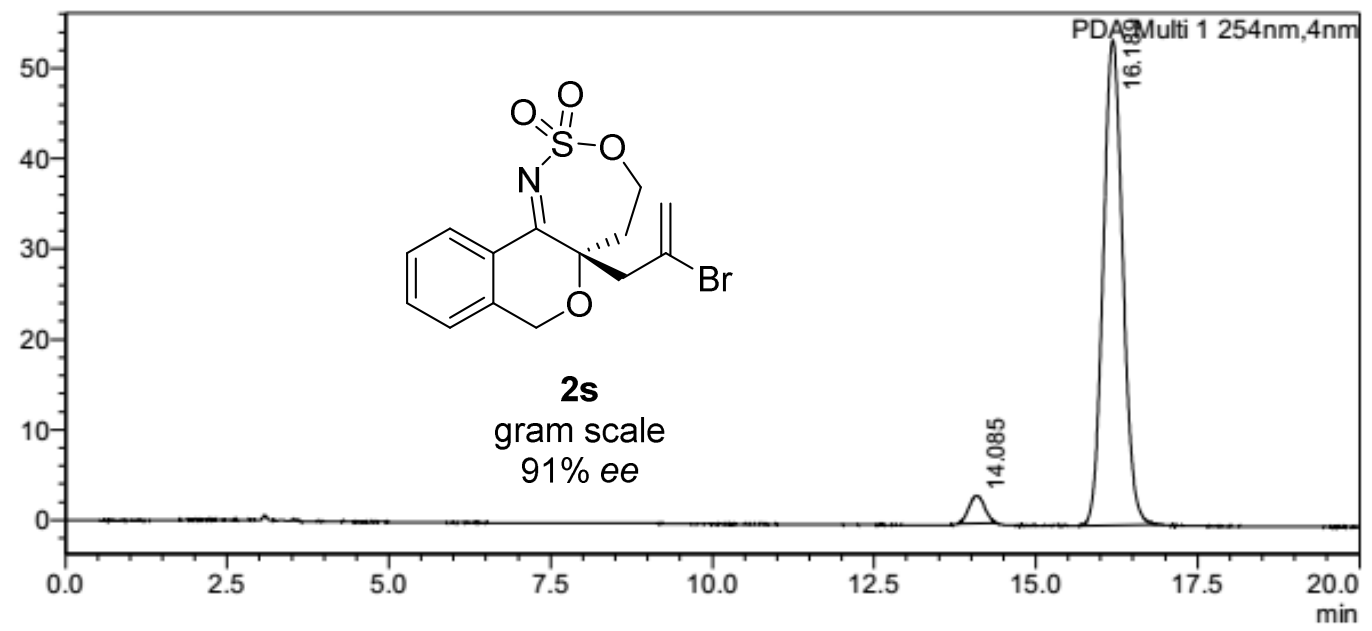

<Peak Table>

PDA Ch1 254nm

\begin{tabular}{|r|r|r|r|r|}
\hline Peak\# & Ret. Time & \multicolumn{1}{c|}{ Area } & Height & \multicolumn{1}{c|}{ Area\% } \\
\hline 1 & 14.085 & 52560 & 3101 & 4.445 \\
\hline 2 & 16.189 & 1129981 & 53650 & 95.555 \\
\hline Tota & & 1182541 & 56751 & 100.000 \\
\hline
\end{tabular}


Condition: hexane $:$-propanol $=90: 10$

Flow rate $=1.0 \mathrm{~mL} / \mathrm{min}, \lambda=254 \mathrm{~nm}$, Daicel Chiralcel IA.

\section{$<$ Chromatogram $>$}

$\mathrm{mAU}$

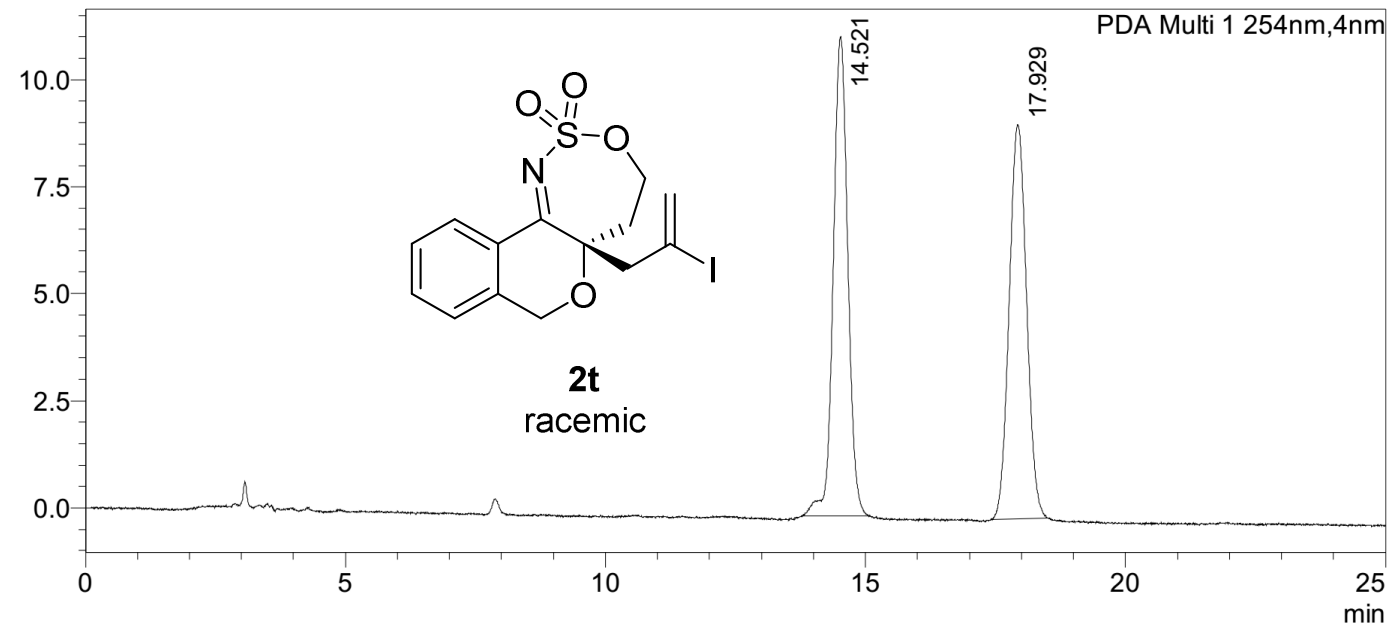

<Peak Table>

PDA Ch1 254nm

\begin{tabular}{|r|r|r|r|r|}
\hline Peak\# Ret. Time & \multicolumn{1}{|c|}{ Area } & Height & \multicolumn{1}{|c|}{ Area\% } \\
\hline 1 & 14.521 & 218990 & 11187 & 50.633 \\
\hline 2 & 17.929 & 213513 & 9203 & 49.367 \\
\hline Total & & 432503 & 20390 & 100.000 \\
\hline
\end{tabular}

\section{$<$ Chromatogram>}

mAU

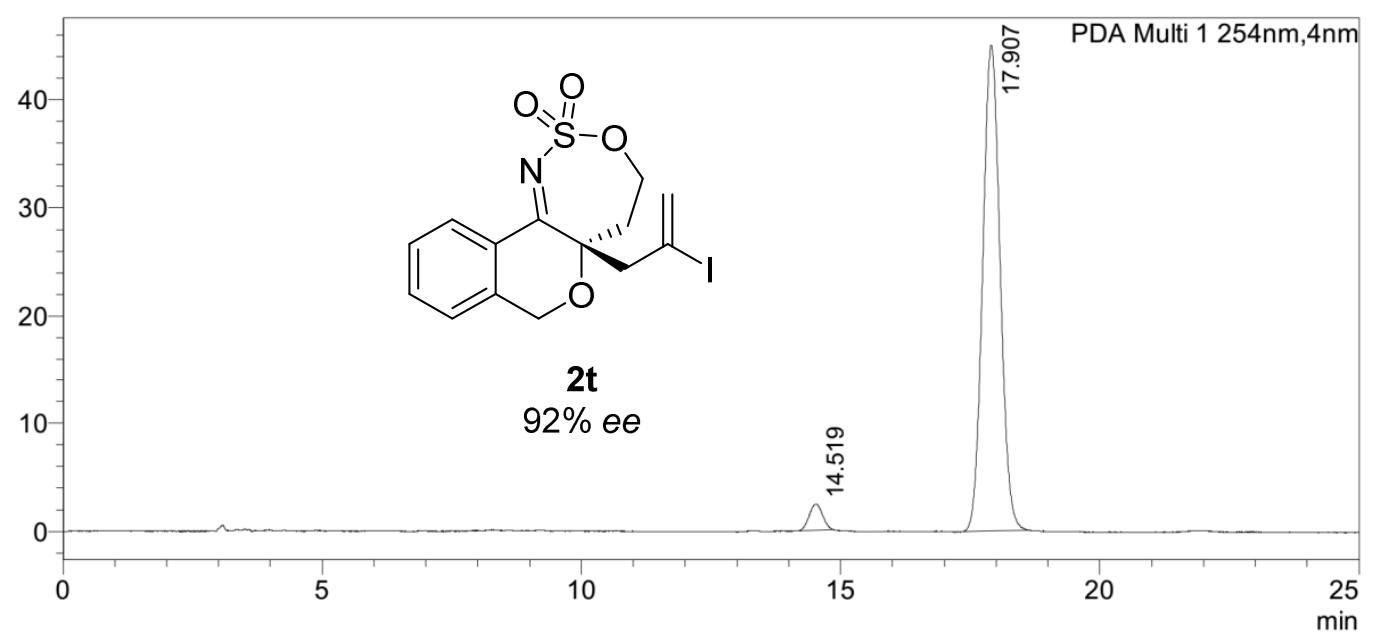

<Peak Table>

PDA Ch1 254nm

\begin{tabular}{|r|r|r|r|r|}
\hline Peak\# & Ret. Time & \multicolumn{1}{c|}{ Area } & Height & \multicolumn{1}{|c|}{ Area\% } \\
\hline 1 & 14.519 & 43453 & 2429 & 3.975 \\
\hline 2 & 17.907 & 1049740 & 45002 & 96.025 \\
\hline Total & & 1093193 & 47431 & 100.000 \\
\hline
\end{tabular}


Condition: hexane $:$ 2-propanol $=80: 20$

Flow rate $=1.0 \mathrm{~mL} / \mathrm{min}, \lambda=254 \mathrm{~nm}$, Daicel Chiralcel IB.

<Chromatogram>

mAU

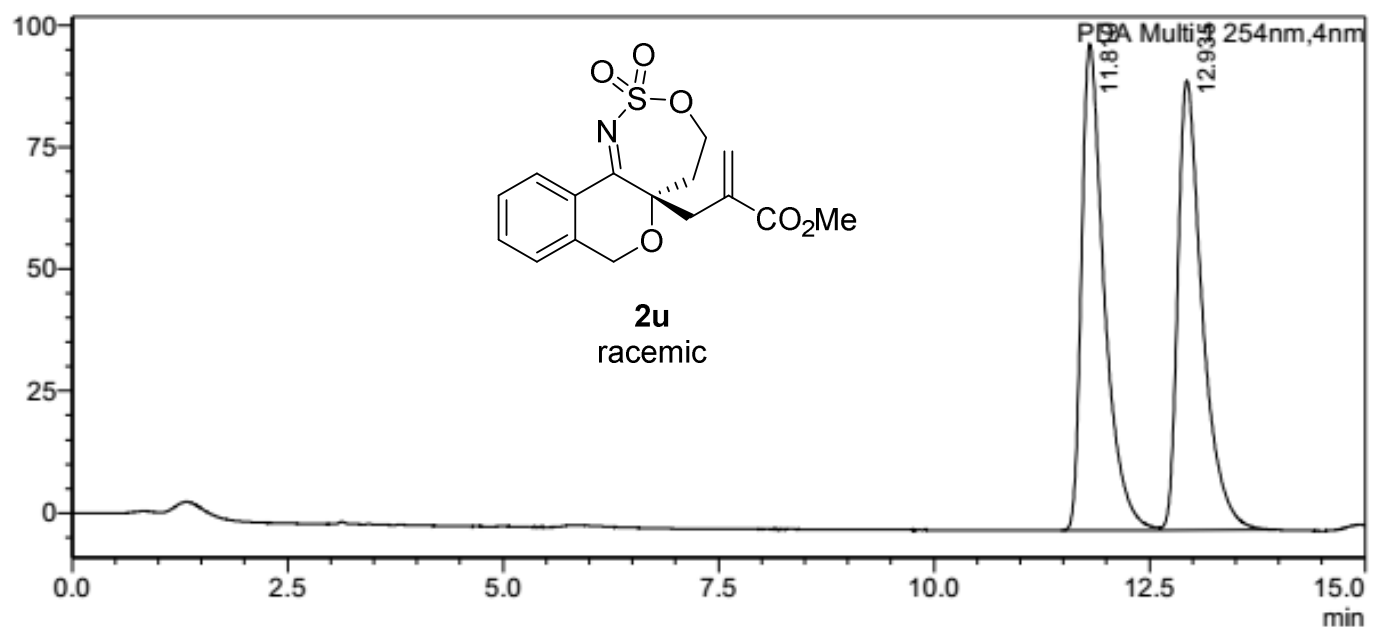

<Peak Table>

PDA Ch1 254nm

\begin{tabular}{|c|c|c|c|c|}
\hline Peak\# & Ret. Time & Area & Height & Area $\%$ \\
\hline 1 & 11.810 & 1829879 & 99730 & 50.026 \\
\hline 2 & 12.935 & 1827985 & 92245 & 49.974 \\
\hline Total & & 3657864 & 191974 & 100.000 \\
\hline
\end{tabular}

$<$ Chromatogram>

$\mathrm{mAU}$

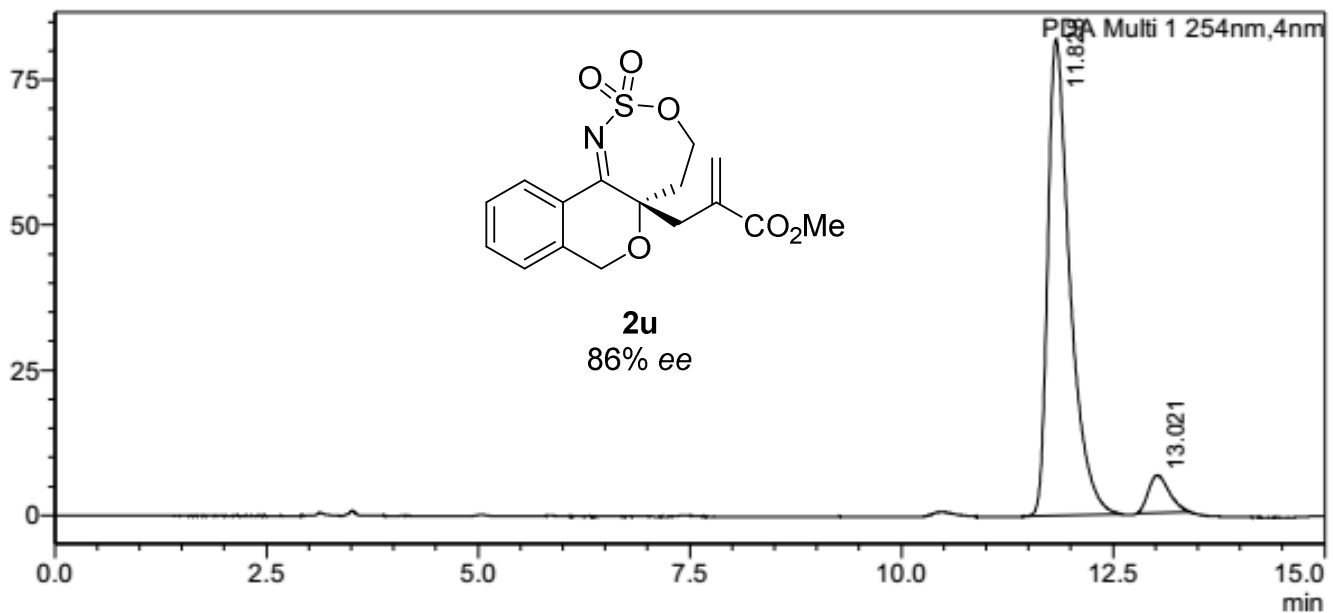

<Peak Table>

PDA Ch1 254nm

\begin{tabular}{|c|c|c|c|c|}
\hline Peak\# & Ret. Time & Area & Height & Area $\%$ \\
\hline 1 & 11.828 & 1496224 & 82063 & 93.030 \\
\hline 2 & 13.021 & 112107 & 6485 & 6.970 \\
\hline Total & & 1608331 & 88548 & 100.000 \\
\hline
\end{tabular}


Condition: hexane $:$ 2-propanol $=90: 10$

Flow rate $=1.0 \mathrm{~mL} / \mathrm{min}, \lambda=254 \mathrm{~nm}$, Daicel Chiralcel IA.

\section{$<$ Chromatogram $>$ \\ mAU}

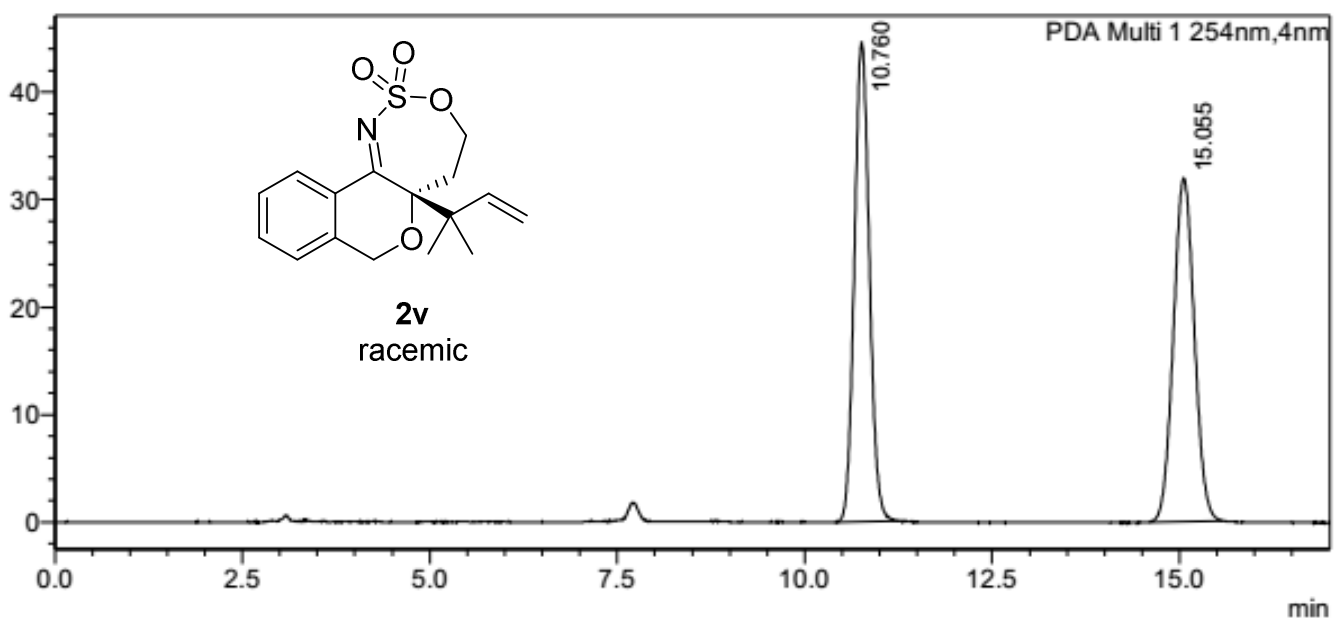

<Peak Table>

PDA Ch1 254nm

\begin{tabular}{|r|r|r|r|r|}
\hline Peak\# & Ret. Time & \multicolumn{1}{c|}{ Area } & Height & \multicolumn{1}{c|}{ Area\% } \\
\hline 1 & 10.760 & 622910 & 44558 & 50.133 \\
\hline 2 & 15.055 & 619610 & 31924 & 49.867 \\
\hline Total & & 1242520 & 76482 & 100.000 \\
\hline
\end{tabular}

\section{$<$ Chromatogram >}

mAU

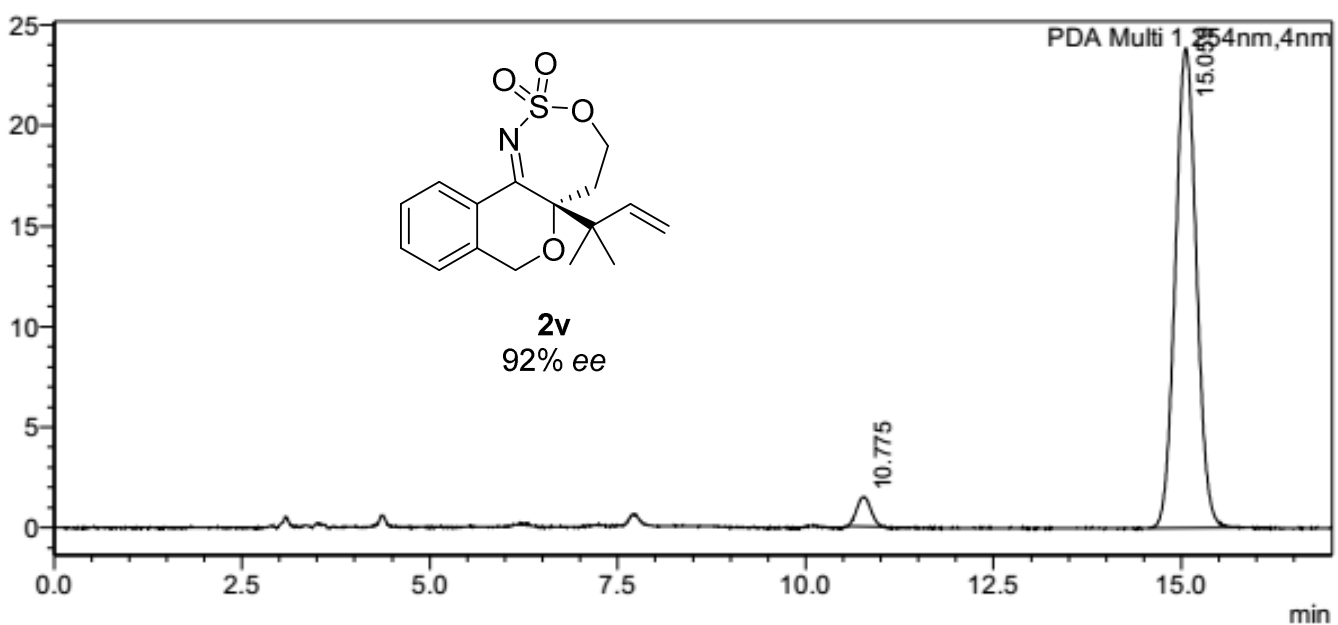

<Peak Table>

PDA Ch1 254nm

\begin{tabular}{|r|r|r|r|r|r|}
\hline Peak\# & Ret. Time & \multicolumn{1}{c|}{ Area } & Height & \multicolumn{1}{|c|}{ Area\% } & \multicolumn{1}{|c|}{ Height\% } \\
\hline 1 & 10.775 & 19236 & 1475 & 3.963 & 5.824 \\
\hline 2 & 15.059 & 466206 & 23842 & 96.037 & 94.176 \\
\hline Total & & 485441 & 25317 & 100.000 & 100.000 \\
\hline
\end{tabular}


Condition: hexane $:$-propanol $=90: 10$

Flow rate $=1.0 \mathrm{~mL} / \mathrm{min}, \lambda=254 \mathrm{~nm}$, Daicel Chiralcel IA.

\section{$<$ Chromatogram $>$}

mAU

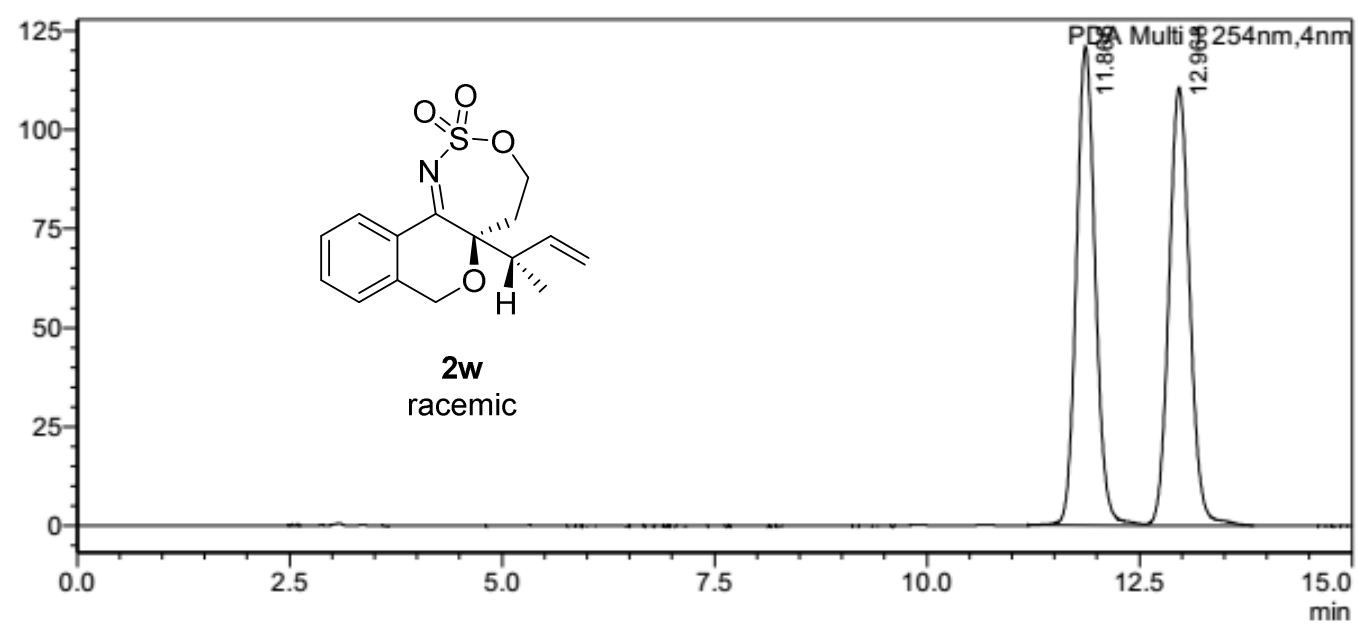

<Peak Table>

PDA Ch1 254nm

\begin{tabular}{|r|r|r|r|r|}
\hline Peak\# & Ret. Time & \multicolumn{1}{|c|}{ Area } & \multicolumn{1}{c|}{ Height } & \multicolumn{1}{c|}{ Area\% } \\
\hline 1 & 11.866 & 1870017 & 120859 & 49.978 \\
\hline 2 & 12.968 & 1871639 & 110585 & 50.022 \\
\hline Total & & 3741656 & 231445 & 100.000 \\
\hline
\end{tabular}

\section{$<$ Chromatogram>}

mAU

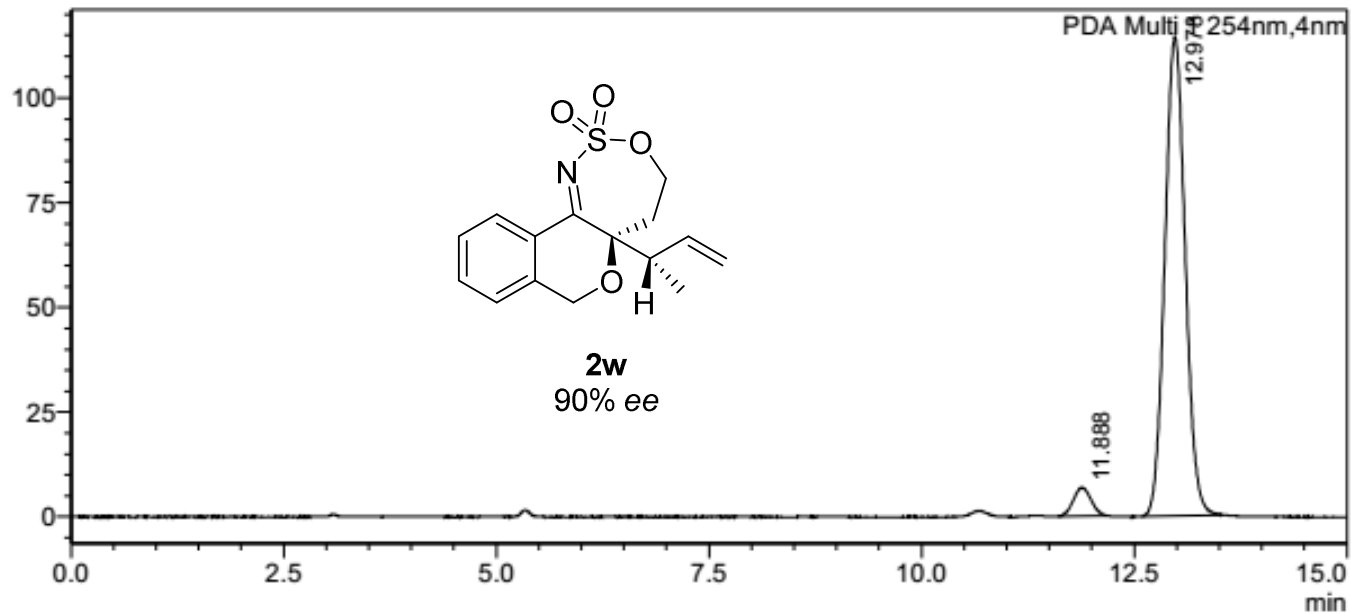

<Peak Table>

PDA Ch1 254nm

\begin{tabular}{|r|r|r|r|r|}
\hline Peak\# Ret. Time & \multicolumn{1}{c|}{ Area } & \multicolumn{1}{c|}{ Height } & \multicolumn{1}{c|}{ Area\% } \\
\hline 1 & 11.888 & 99284 & 6778 & 4.958 \\
\hline 2 & 12.978 & 1903333 & 114389 & 95.042 \\
\hline Total & & 2002617 & 121167 & 100.000 \\
\hline
\end{tabular}


Condition: hexane $:$ 2-propanol $=90: 10$

Flow rate $=1.0 \mathrm{~mL} / \mathrm{min}, \lambda=254 \mathrm{~nm}$, Daicel Chiralcel IA.

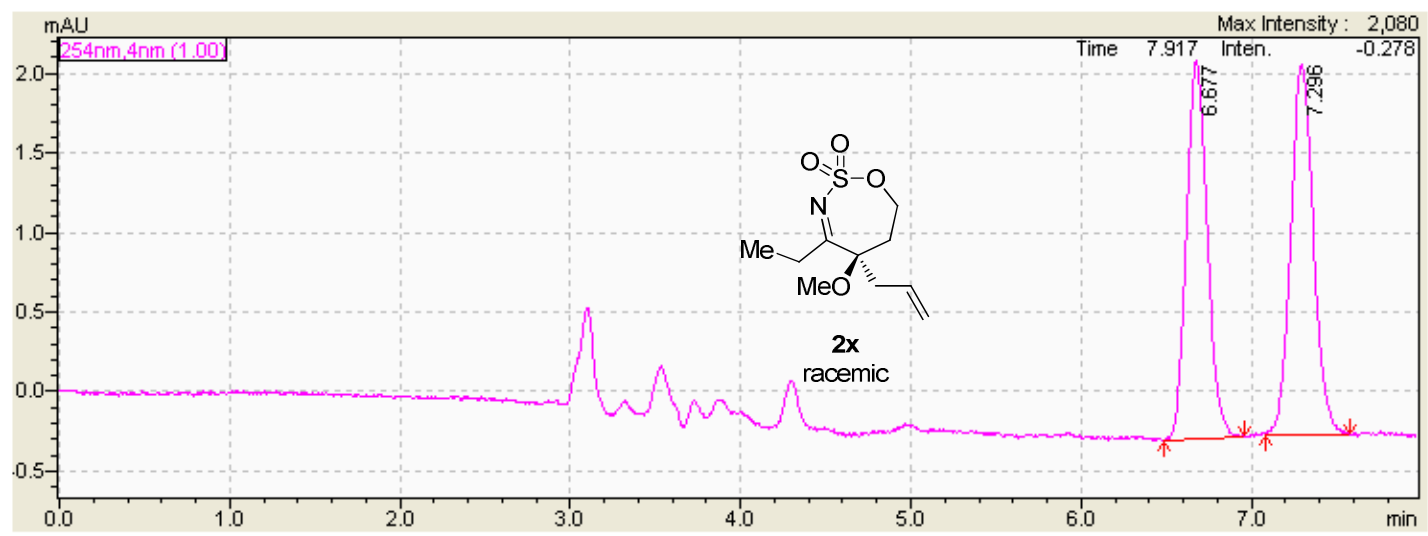

<Peak Table>

\begin{tabular}{|c|c|c|c|c|}
\hline & \multicolumn{3}{|c|}{ Peak\#Ret. Time } \\
\hline 1 & 6.674 & 22003 & 2564 & 48.671 \\
\hline 2 & 7.291 & 23204 & 2539 & 51.329 \\
\hline $\mathrm{To}$ & & 45207 & 5103 & 100.000 \\
\hline
\end{tabular}

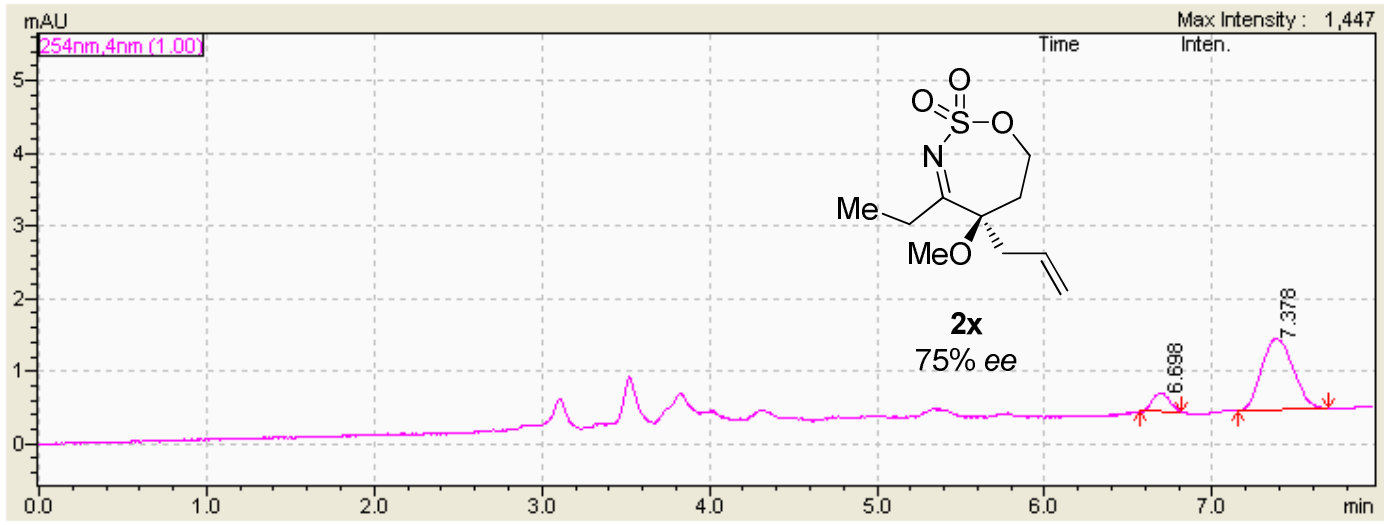

<Peak Table>

PDA Ch1 254nm

\begin{tabular}{|r|r|r|r|r|}
\hline Peak\# & Ret. Time & \multicolumn{1}{|c|}{ Area } & \multicolumn{1}{c|}{ Height } & \multicolumn{1}{c|}{ Area\% } \\
\hline 1 & 6.698 & 1799 & 250 & 12.276 \\
\hline 2 & 7.378 & 12856 & 978 & 87.724 \\
\hline Total & & 14655 & 1228 & 100.000 \\
\hline
\end{tabular}


Condition: hexane $:$ 2-propanol $=90: 10$

Flow rate $=1.0 \mathrm{~mL} / \mathrm{min}, \lambda=254 \mathrm{~nm}$, Daicel Chiralcel IA

\section{$<$ Chromatogram >}

mAU

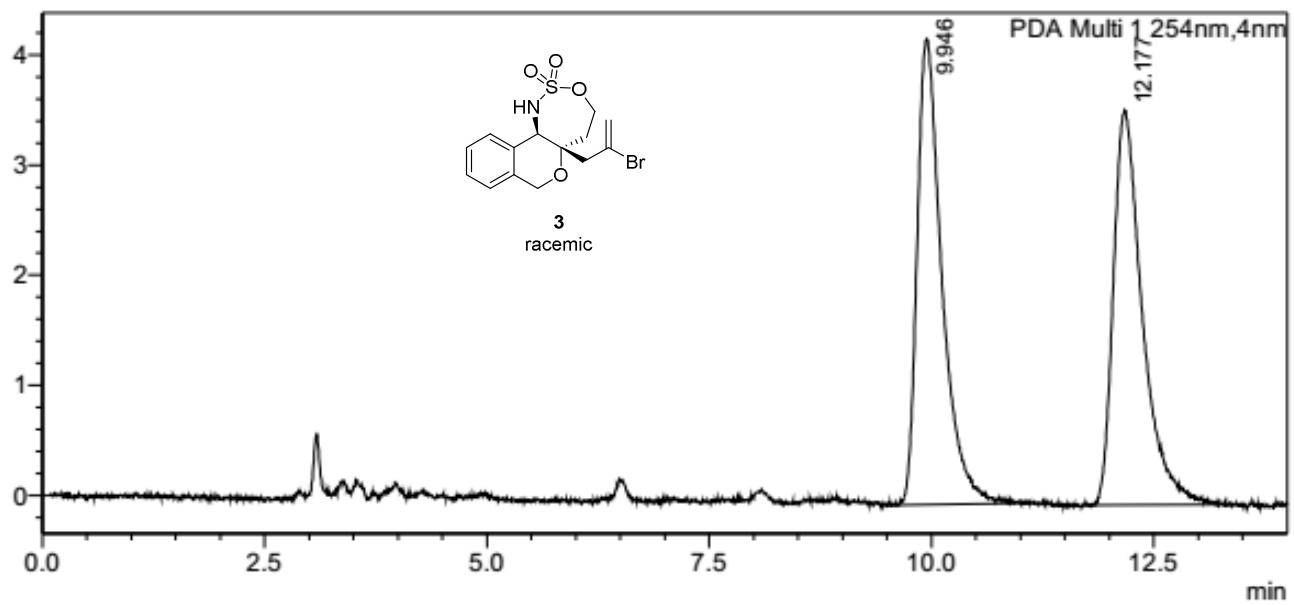

<Peak Table>

PDA Ch1 254nm

\begin{tabular}{|r|r|r|r|r|}
\hline Peak\# & Ret. Time & \multicolumn{1}{c|}{ Area } & Height & \multicolumn{1}{c|}{ Area\% } \\
\hline 1 & 9.946 & 82301 & 4224 & 50.237 \\
\hline 2 & 12.177 & 81523 & 3582 & 49.763 \\
\hline Total & & 163824 & 7806 & 100.000 \\
\hline
\end{tabular}

$<$ Chromatogram>

$\mathrm{mAl}$

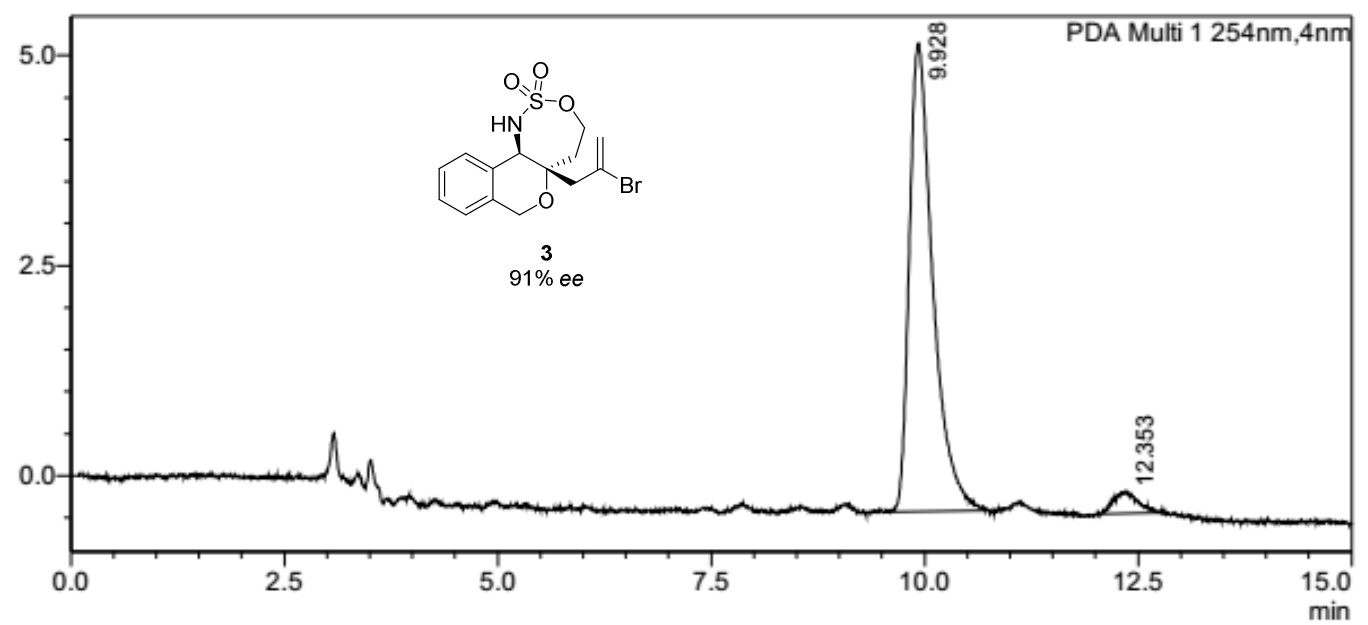

<Peak Table>

PDA Ch1 254nm

\begin{tabular}{|r|r|r|r|r|}
\hline Peak\# & Ret. Time & \multicolumn{1}{c|}{ Area } & Height & \multicolumn{1}{c|}{ Area\% } \\
\hline 1 & 9.928 & 107092 & 5570 & 95.400 \\
\hline 2 & 12.353 & 5164 & 259 & 4.600 \\
\hline Total & & 112256 & 5829 & 100.000 \\
\hline
\end{tabular}


Condition: hexane $:$ 2-propanol $=90: 10$

Flow rate $=1.0 \mathrm{~mL} / \mathrm{min}, \lambda=254 \mathrm{~nm}$, Daicel Chiralcel IA

\section{$<$ Chromatogram >}

mAU

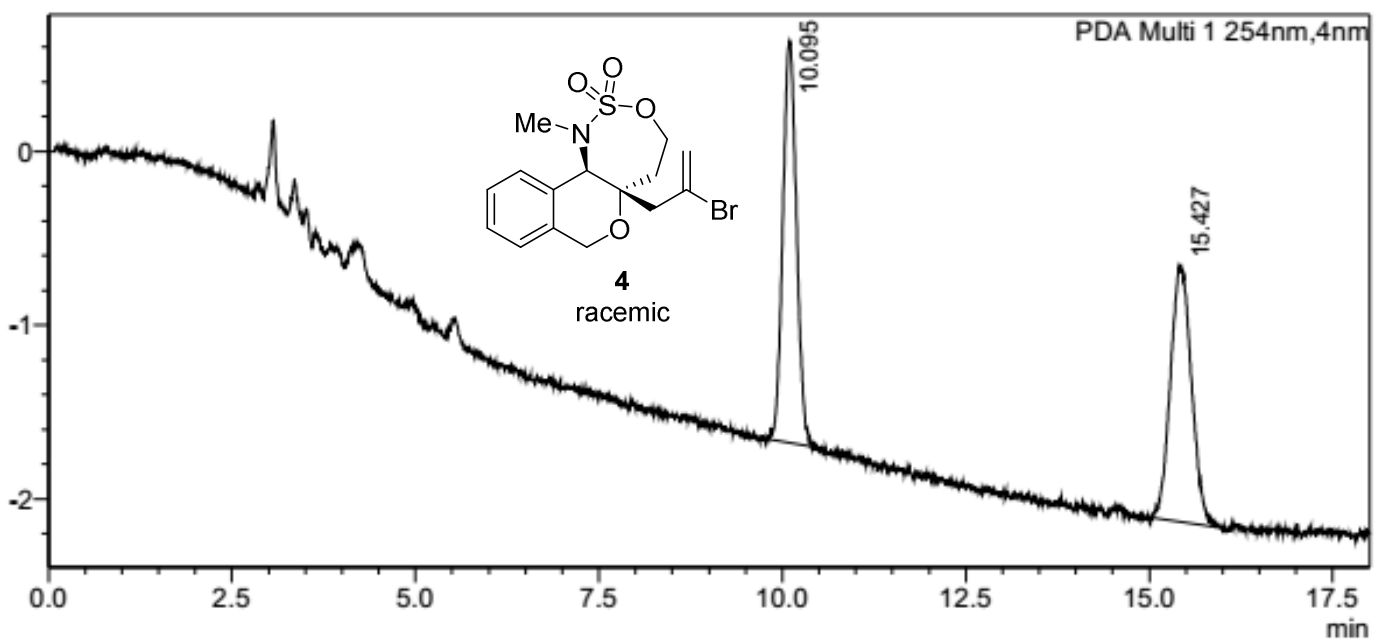

<Peak Table>

PDA Ch1 254nm

\begin{tabular}{|r|r|r|r|r|}
\hline Peak\# & Ret. Time & \multicolumn{1}{c|}{ Area } & Height & \multicolumn{1}{c|}{ Area\% } \\
\hline 1 & 10.095 & 29975 & 2303 & 50.308 \\
\hline 2 & 15.427 & 29608 & 1479 & 49.692 \\
\hline Total & & 59584 & 3781 & 100.000 \\
\hline
\end{tabular}

\section{$<$ Chromatogram $>$}

$\mathrm{mAU}$

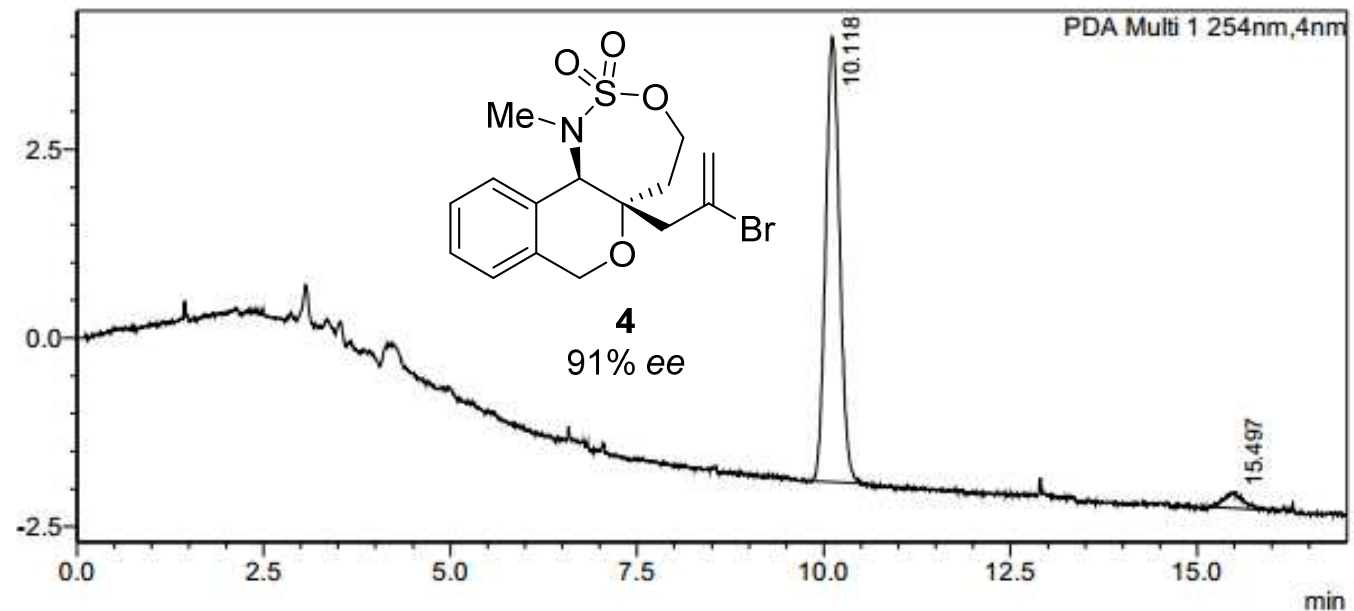

<Peak Table>

PDA Ch1 254nm

\begin{tabular}{|c|c|c|c|c|}
\hline Peak\# & let. Time & Area & Height & Area $\%$ \\
\hline 1 & 10.118 & 76661 & 5890 & 95.522 \\
\hline 2 & 15.497 & 3594 & 203 & 4.478 \\
\hline Total & & 80255 & 6093 & 100.000 \\
\hline
\end{tabular}


Condition: hexane $:$ 2-propanol $=90: 10$

Flow rate $=1.0 \mathrm{~mL} / \mathrm{min}, \lambda=254 \mathrm{~nm}$, Daicel Chiralcel IA

\section{$<$ Chromatogram >}

mAU

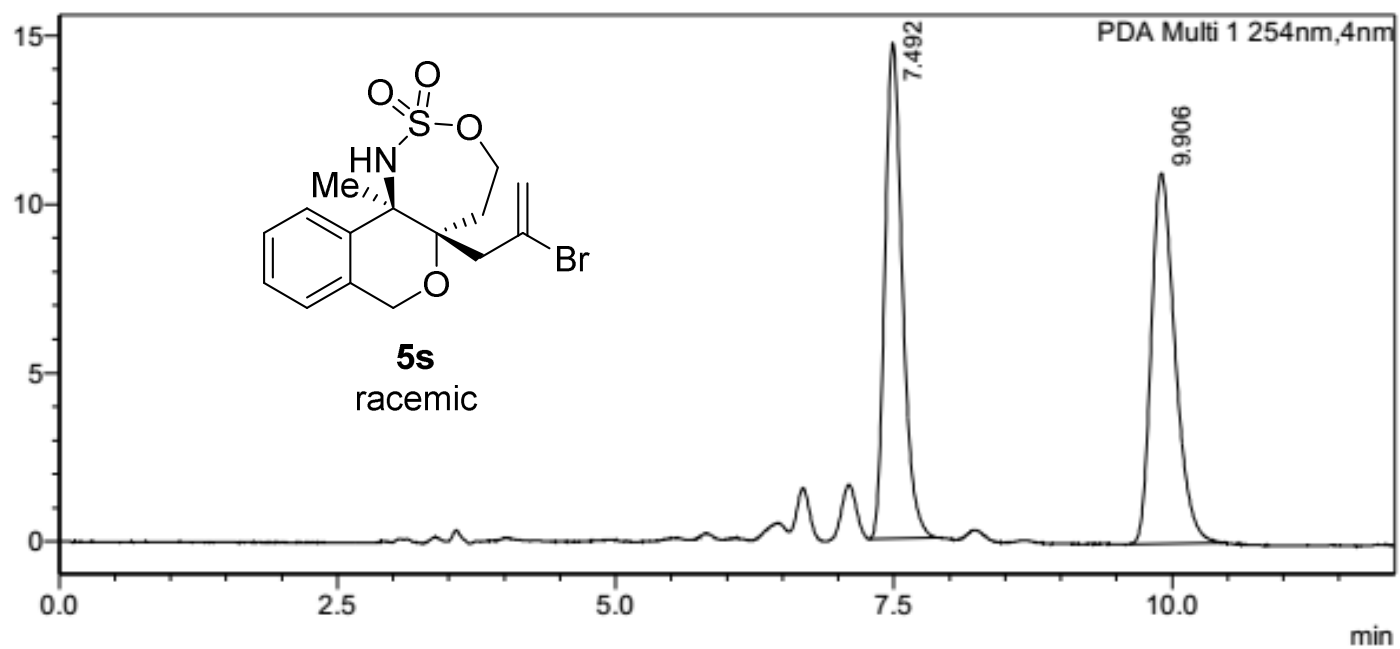

<Peak Table>

PDA Ch1 254nm

\begin{tabular}{|r|r|r|r|r|}
\hline Peak\# & Ret. Time & \multicolumn{1}{c|}{ Area } & Height & \multicolumn{1}{c|}{ Area\% } \\
\hline 1 & 7.492 & 161033 & 14685 & 49.714 \\
\hline 2 & 9.906 & 162888 & 10969 & 50.286 \\
\hline Total & & 323921 & 25654 & 100.000 \\
\hline
\end{tabular}

\section{$<$ Chromatogram $>$}

mAU

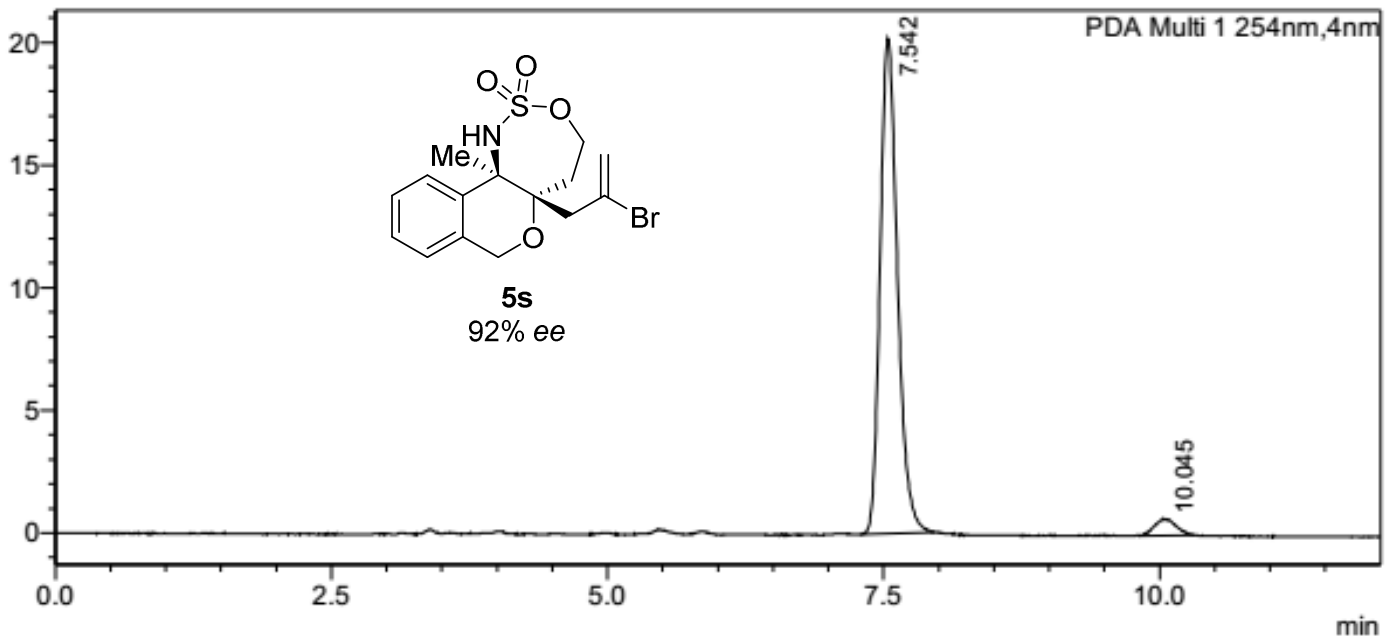

\section{<Peak Table>}

PDA Ch1 254nm

\begin{tabular}{|c|c|c|c|c|}
\hline Peak\# & Ret. Time & Area & Height & Area\% \\
\hline 1 & 7.542 & 220875 & 20189 & 96.018 \\
\hline 2 & 10.045 & 9160 & 669 & 3.982 \\
\hline Total & & 230035 & 20858 & 100.000 \\
\hline
\end{tabular}


Condition: hexane $:$-propanol $=90: 10$

Flow rate $=1.0 \mathrm{~mL} / \mathrm{min}, \lambda=254 \mathrm{~nm}$, Daicel Chiralcel IA

$<$ Chromatogram>

mAU

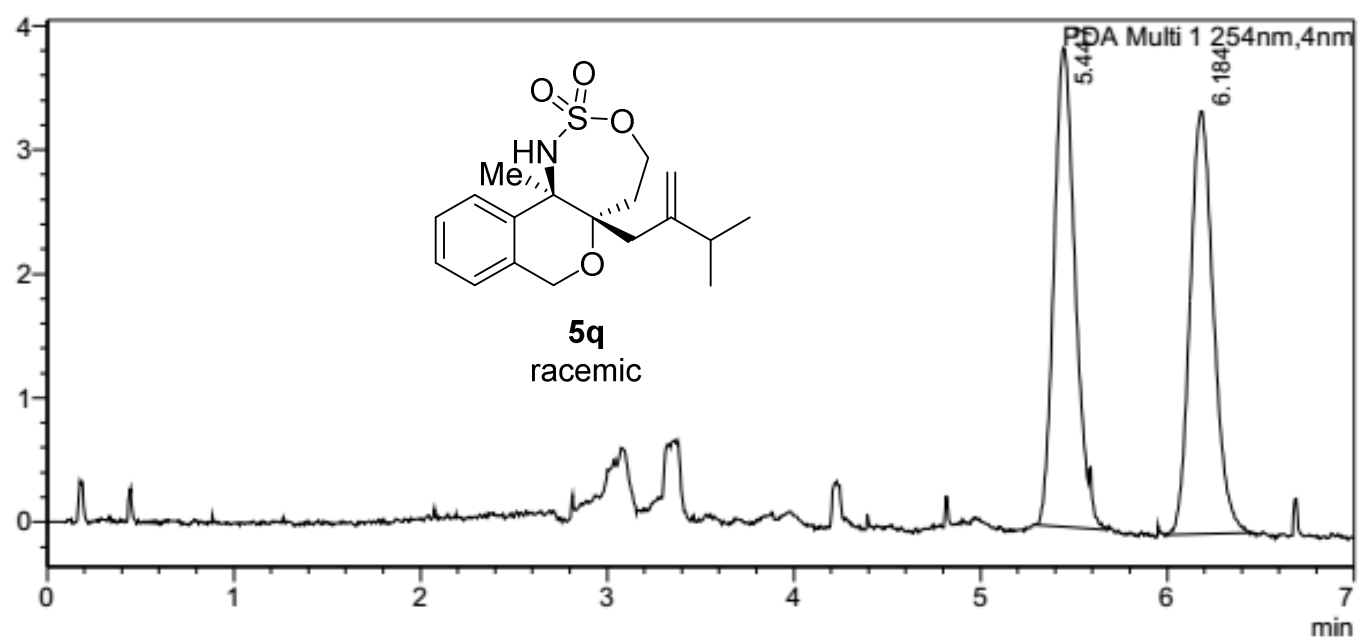

<Peak Table>

PDA Ch1 254nm

\begin{tabular}{|c|c|c|c|c|}
\hline Peak\# & Ret. Time & Area & Height & Area $\%$ \\
\hline 1 & 5.447 & 30504 & 3861 & 50.604 \\
\hline 2 & 6.184 & 29775 & 3411 & 49.396 \\
\hline Total & & 60278 & 7271 & 100.000 \\
\hline
\end{tabular}

$<$ Chromatogram >

mAU

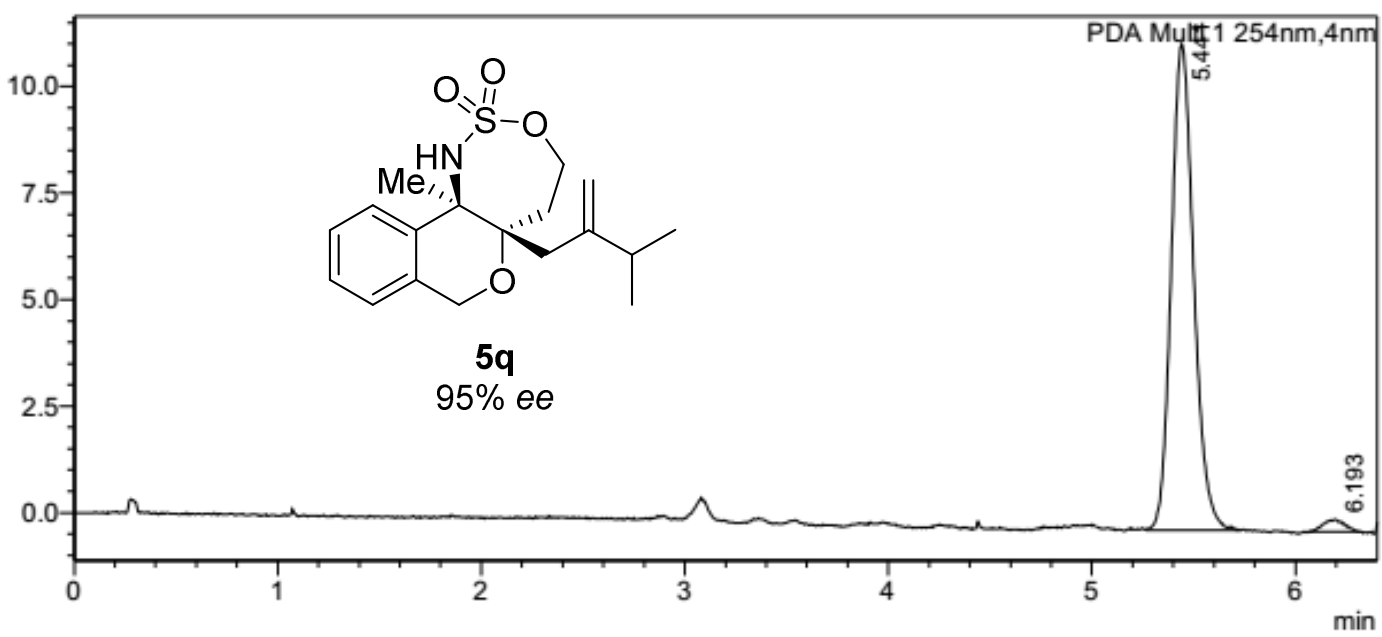

$<$ Peak Table>

PDA Ch1 254nm

\begin{tabular}{|r|r|r|r|r|}
\hline Peak\# & Ret. Time & \multicolumn{1}{c|}{ Area } & \multicolumn{1}{c|}{ Height } & \multicolumn{1}{c|}{ Area\% } \\
\hline 1 & 5.441 & 87848 & 11416 & 97.643 \\
\hline 2 & 6.193 & 2121 & 288 & 2.357 \\
\hline Total & & 89969 & 11704 & 100.000 \\
\hline
\end{tabular}


Condition: hexane $:$-propanol $=90: 10$

Flow rate $=1.0 \mathrm{~mL} / \mathrm{min}, \lambda=254 \mathrm{~nm}$, Daicel Chiralcel IA

\section{$<$ Chromatogram $>$}

mAU

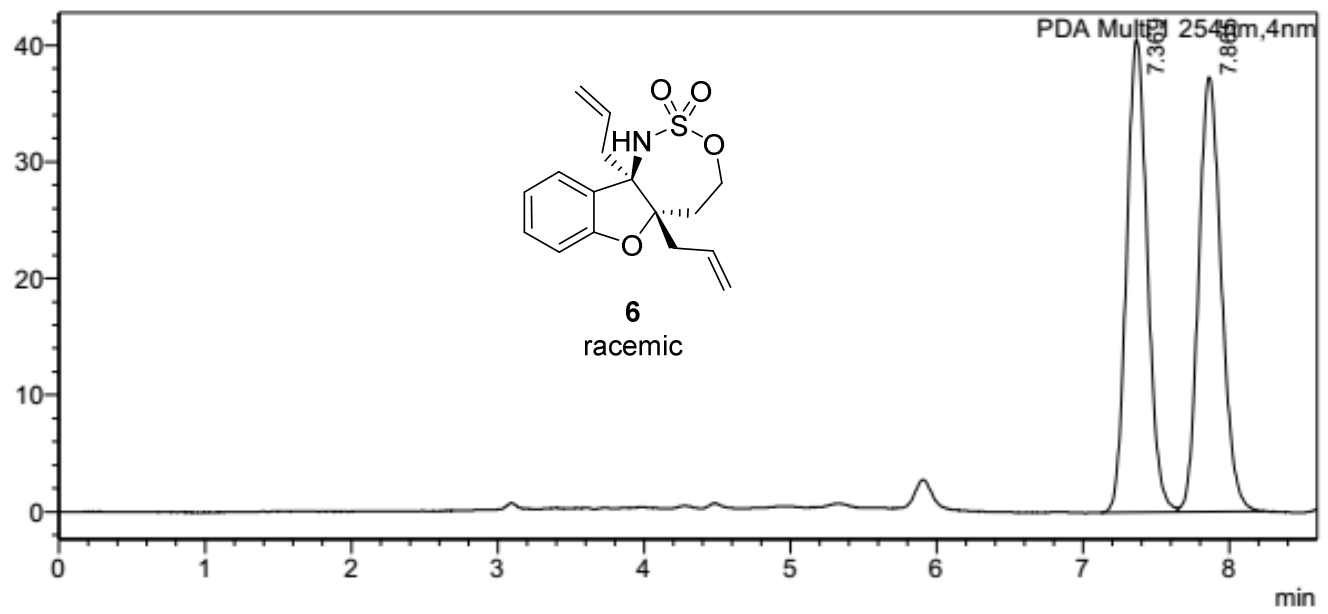

<Peak Table>

PDA Ch1 254nm

\begin{tabular}{|c|c|c|c|c|}
\hline Peak\# & Ret. Time & Area & Height & Area $\%$ \\
\hline 1 & 7.369 & 401156 & 40559 & 49.959 \\
\hline 2 & 7.865 & 401811 & 37227 & 50.04 \\
\hline Total & & 802967 & 77786 & 100.00 \\
\hline
\end{tabular}

$<$ Chromatogram>

$\mathrm{mAU}$

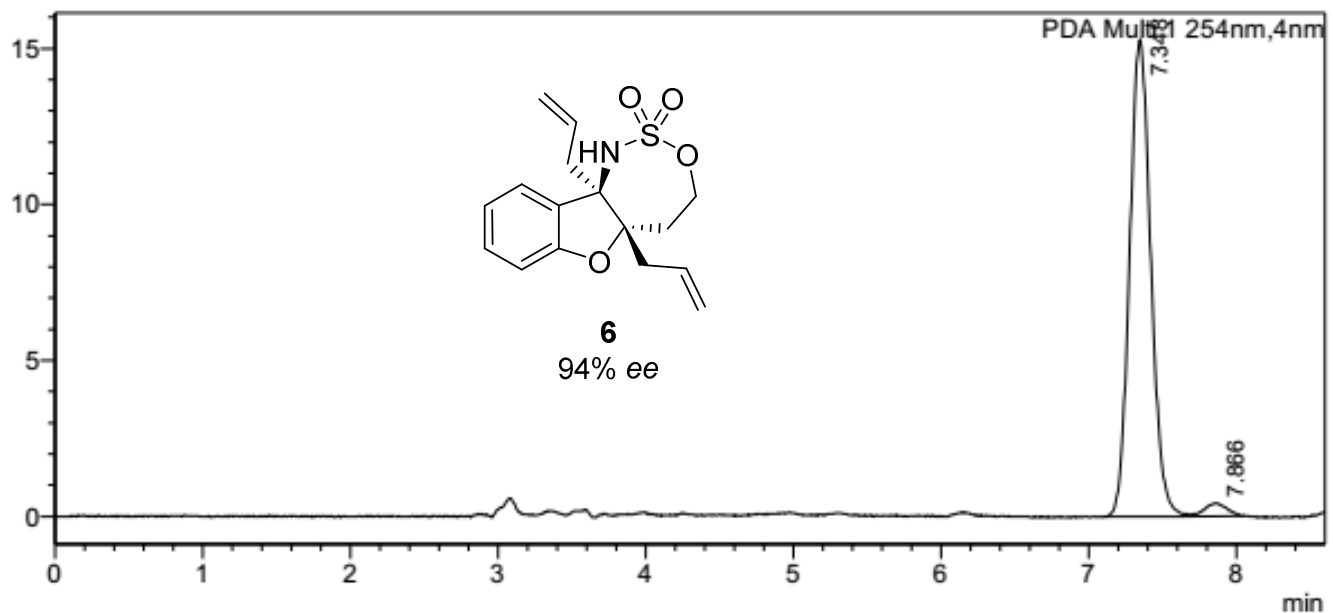

<Peak Table>

PDA Ch1 254nm

\begin{tabular}{|r|r|r|r|r|}
\hline Peak\# & Ret. Time & \multicolumn{1}{c|}{ Area } & \multicolumn{1}{c|}{ Height } & \multicolumn{1}{c|}{ Area\% } \\
\hline 1 & 7.348 & 152506 & 15293 & 97.154 \\
\hline 2 & 7.866 & 4467 & 410 & 2.846 \\
\hline Total & & 156973 & 15703 & 100.000 \\
\hline
\end{tabular}




\section{Crystallographic Data for $2 d$.}

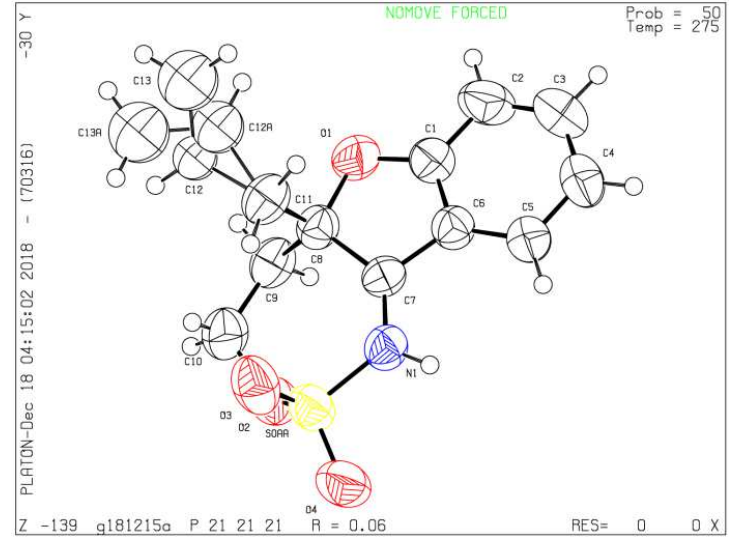

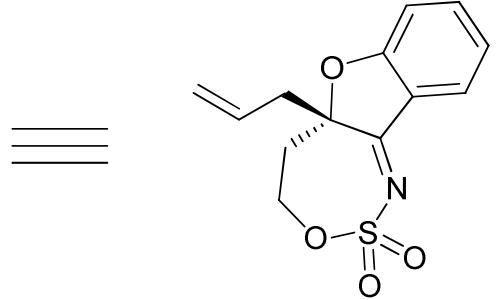

(S)-2d (CCDC: 1886681)

\section{Datablock: g181215a}

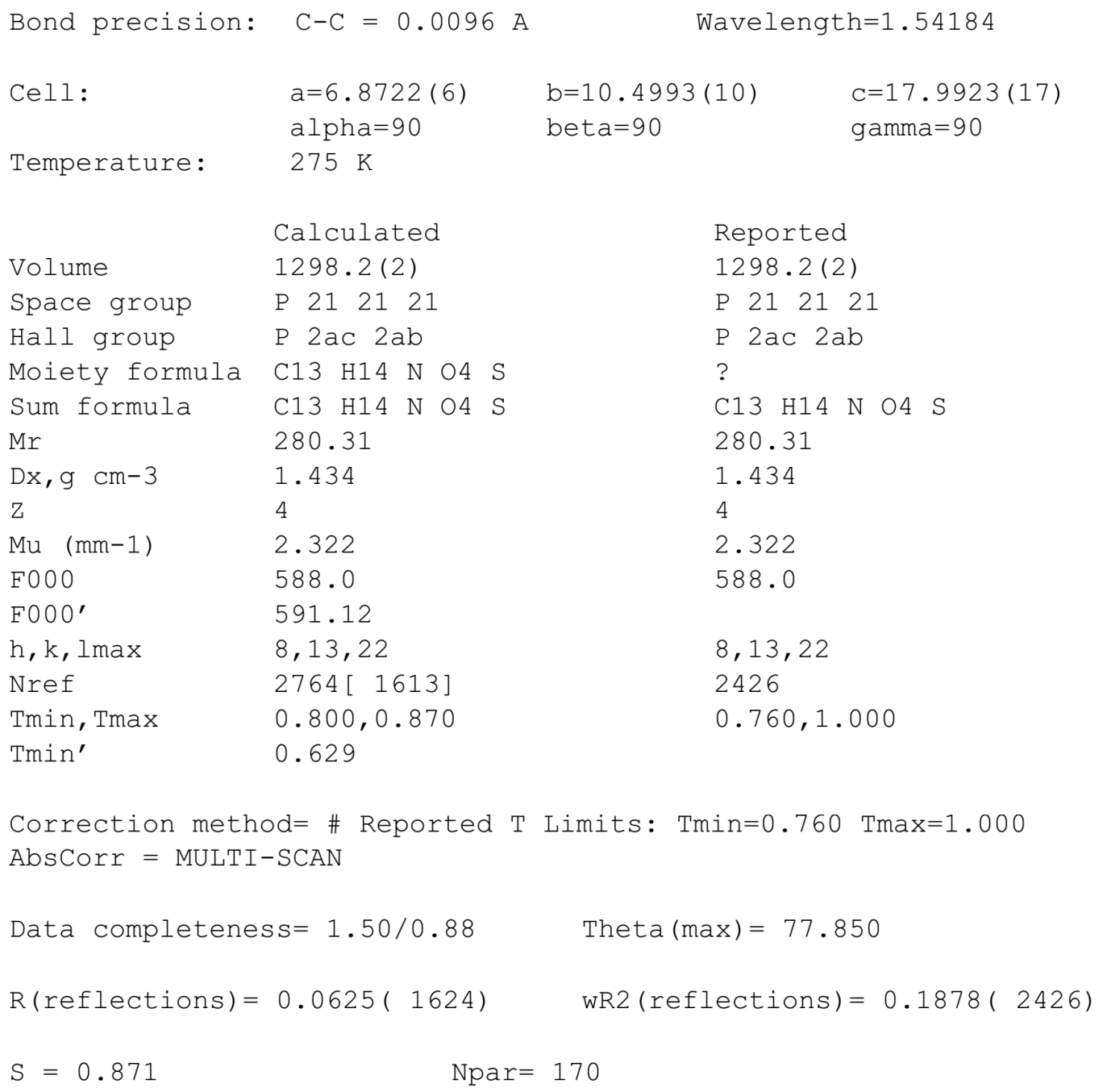

Correction method= \# Reported T Limits: Tmin=0.760 Tmax=1.000 AbsCorr $=$ MULTI-SCAN 